\title{
WestVirginiaUniversity
}

THE RESEARCH REPOSITORY @ WVU

Graduate Theses, Dissertations, and Problem Reports

2004

\section{Modeling and real-time feedback control of MEMS device}

Limin Wang

West Virginia University

Follow this and additional works at: https://researchrepository.wvu.edu/etd

\section{Recommended Citation}

Wang, Limin, "Modeling and real-time feedback control of MEMS device" (2004). Graduate Theses, Dissertations, and Problem Reports. 2156.

https://researchrepository.wvu.edu/etd/2156

This Dissertation is protected by copyright and/or related rights. It has been brought to you by the The Research Repository @ WVU with permission from the rights-holder(s). You are free to use this Dissertation in any way that is permitted by the copyright and related rights legislation that applies to your use. For other uses you must obtain permission from the rights-holder(s) directly, unless additional rights are indicated by a Creative Commons license in the record and/ or on the work itself. This Dissertation has been accepted for inclusion in WVU Graduate Theses, Dissertations, and Problem Reports collection by an authorized administrator of The Research Repository @ WVU.

For more information, please contact researchrepository@mail.wvu.edu. 


\title{
Modeling and Real-Time Feedback Control of MEMS Device
}

\author{
Limin Wang \\ Dissertation submitted to \\ The College of Engineering And Mineral Resources \\ at West Virginia University \\ In partial fulfillment of the requirements for the degree of \\ Doctor of Philosophy \\ in \\ Electrical Engineering
}

Advisory and Examining Committee Members:

Parviz Famouri, Ph.D. Chair

Larry A. Hornak, Ph.D.

Mark A. Jerabek, Ph.D.

Matthew C. Valenti, Ph.D.

Marcello R. Napolitano, Ph.D.

Lane Department of Computer Science and Electrical Engineering

Morgantown, West Virginia

2004

Keywords: MEMS, System Identification, Real-time Feedback Control 


\title{
ABSTRACT \\ Modeling and Real-Time Feedback Control of MEMS Device
}

\author{
Limin Wang
}

Applying closed-loop control to a MEMS devices not only can handle the abnormal behaviors caused by manufactory imprecision or device failure, enabling MEMS devices to survive in critical conditions, but also can increase the application where MEMS devices are used to drive components under varying load conditions. This study mainly focuses on the effort of closed-loop control on the Lateral Comb Resonator (LCR) MEMS device. The success of closed-loop control has been achieved on lateral comb resonator with novel integrated through wafer optical monitoring technique [1].

Availability of a system model and feedback signals are mandatory conditions for the implementation of closed-loop control. Because of the fabrication process tolerance, the parameters of the Lateral Comb Resonator (LCR)'s model, especially the damping parameter $\beta$, cannot be determined accurately based merely on theoretical analysis. Therefore, performing system identification through experiments can be a tool to verify the system model. Three different system identification methods in both the time domain and frequency domain have been implemented, and the results agree.

Noise analysis on the optical monitoring signal shows that at least $90 \%$ of the noise in the signal is due to the optical monitoring setup, and the simulation shows that both a wavelet thresholding method and frequency domain low pass filter are efficient in removing this Gaussian distributed noise.

Different designs are developed to monitor the LCR, both for single opening and grating structure LCRs, resulting in monitoring signals that are very different in nature. The optical monitoring signal for single opening device is highly correlated with the LCR's shuttle position. After removing the noise, this signal can be used directly as a feedback signal to perform closed-loop control on the shuttle for damping shock effect or to perform stroke-length control on the shuttle [2]. The optical monitoring signal for the grating structure LCR is a kind of frequency modulation of the shuttle's displacement. Based on this signal, both position and velocity signals can be reconstructed in real time $[1,3]$. Even though the reconstructed position and velocity signals will inherit and even amplify all the noises that exists in the optical monitoring signal, with this method acceptable performance has been achieved in the tracking control experiment of the LCR's shuttle. In this experiment, the newly designed model reference adaptive fuzzy sliding controller (MFSC) effectively minimized the side effects caused by either signal noise or imprecision of the system model. Furthermore, experimental success on force estimation has been achieved based on this signal reconstruction method [4].

The signal reconstruction method, which can decouple the noise from the optical signal, has been implemented. $2 \mu \mathrm{m}$ 's resolution can be achieved with the current single beam optical monitoring method. The resolution can be improved several times with the implementation of multi-beam optical monitoring in the near future. 


\section{Acknowledgements}

I would like to this opportunity to express my gratitude to Dr. Parviz Famouri, a great man and excellent teacher and advisor, for giving me the opportunity to work on this interesting project, and for providing me with the guidance, support and resource necessary for accomplishing my research objectives.

My sincere appreciated also goes to my committee members, Dr. Larry A. Hornak, Dr. Mark A. Jerabek, Dr. Matthew C. Valenti, and Dr. Marcello R. Napolitano, for the benefit of their collective wisdom, helpful comments, and generously sharing their tiem and talents to guide me during the course of my research.

I would like to give thanks to current and previous members of the WVU MEMS research group, especially Dr. Jingdong Chen, Dr. my Dawson, Lakshmi Deepasree, Afshin Izadian, Willam McCormic, Raghuveer Medipalli, Swetha Katipalli, and Scott Rittenhouse, for their assistance, encourage and tolerance.

Finally, and most importantly, I would like to delicate this work to my dear husband Ligong, his love and support helped me to bring this work and my degree to completion. I would also like to give thanks to my parents and sister for encouraging me in pursing my education. I love you all very much. 


\section{Contents}

ABSTRACT.................................................................................................................. II

ACKNOWLEDGEMENTS ..............................................................................................II

CONTENTS..................................................................................................................... IV

CHAPTER 1 INTRODUCTION............................................................................. 1

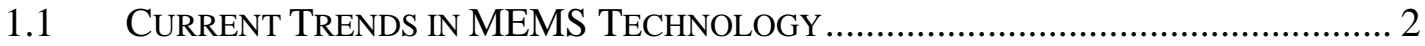

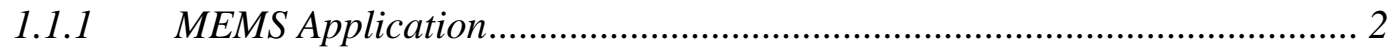

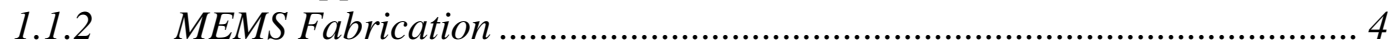

1.1.3 MEMS Failure and Reliability.............................................................. 8

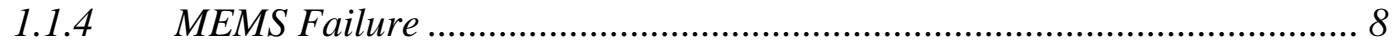

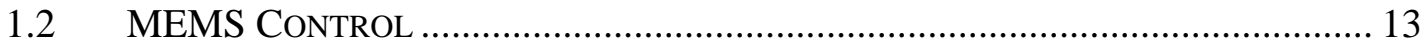

1.3 InTEgrated Through WAfER Monitoring Method..................................... 15

1.4 Device Introduction (Single Opening \& Grating STRUCture) ................ 16

CHAPTER 2 NOISE ANALYSIS AND SIGNAL FILTERING ............................ 20

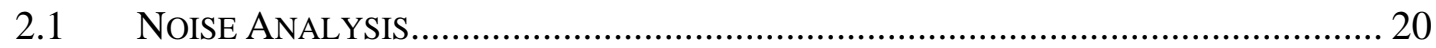

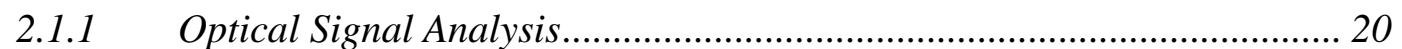

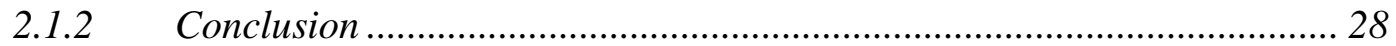

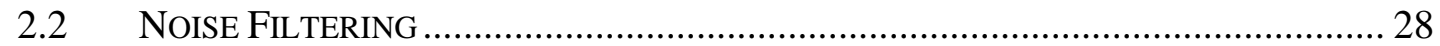

2.2.1 Brief Introduction on Noise Reduction .................................................... 28

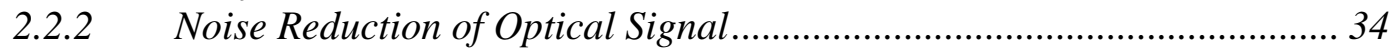

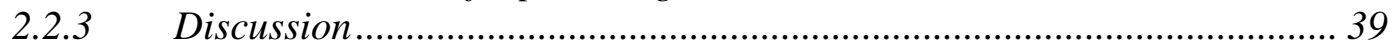

CHAPTER 3 SYSTEM MODELING ..................................................................... 41

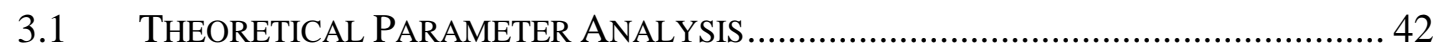

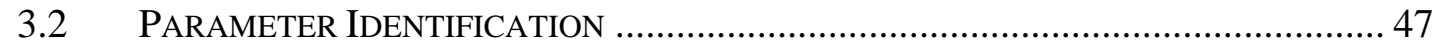

3.2.1 Time Domain System Identification ........................................................... 47

3.2.2 Frequency Domain System Identification .............................................. 53

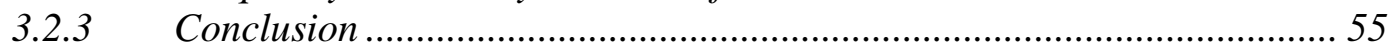

3.3 EXPERIMENTAL IMPLEMENTATION OF PARAMETER IDENTIFICATION................... 56

3.3.1 Experimental system identification on single opening device ....................5 56

3.3.2 Experimental system identification on grating structure device................. 70

3.3.3 Experimental system identification on a non-linear device ...................... 76

\section{CHAPTER 4 FEEDBACK CONTROL ON SINGLE OPENING MEMS}

LATERAL COMB RESONATOR ............................................................................... 80

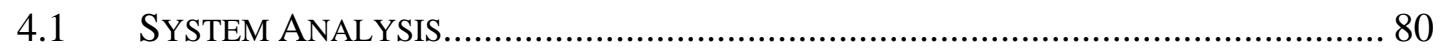

4.2 ACTIVE CONTROL OF DAMPING SHOCK EFFECTS............................................. 81

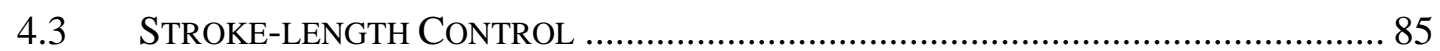

CHAPTER 5 FEEDBACK CONTROL ON GRATING STRUCTURE MEMS

LATERAL COMB RESONATOR ................................................................................90 
5.1 RECONSTRUCTION POSITION SignAL FROM OptICAL SigNAL............................. 91

5.1.1 On-line position signal reconstruction approach \#1 ................................. 92

5.1.2 On-line position signal reconstruction approach \#2 ............................... 100

5.2 EXPERIMENTAL ESTIMATION OF ELECTRICAL FORCE …..................................... 103

5.3 CLOSED-LOOP TRACKING CONTROL ......................................................... 109

CHAPTER 6 CONCLUSIONS AND FUTURE CONSIDERATION................... 115

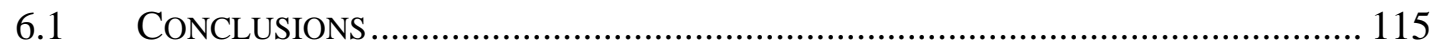

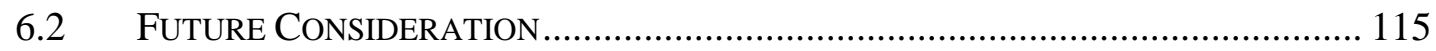

APPENDIX A SOURCE CODE \& SIMULINK MODEL ................................ 117

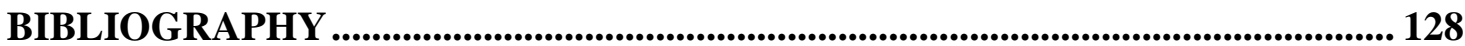




\section{Chapter 1 Introduction}

Micro-Electro-Mechanical System (MEMS) is the combination of electrical and mechanical devices built at micron-scale using microfabrication technology, which is originated in semiconductor industry. Equivalent terms for MEMS are microsystem (preferred in Europe) and micromachine (preferred in Japan). With MEMS technology, the size of many types of sensors, actuators and systems, when compared to their macroscopic versions, can be reduced by several orders of magnitude. The typical size of MEMS devices is usually measured in micrometers or even nanometers. MEMS has a prospective future in both civilian and military applications. It is widely used in mechanical maintenance systems, mini-plants, biomedical applications, and miniature aircraft and satellites. About 100 million MEMS components are sold annually [5]. Figure 1-1 shows some examples of MEMS devices.

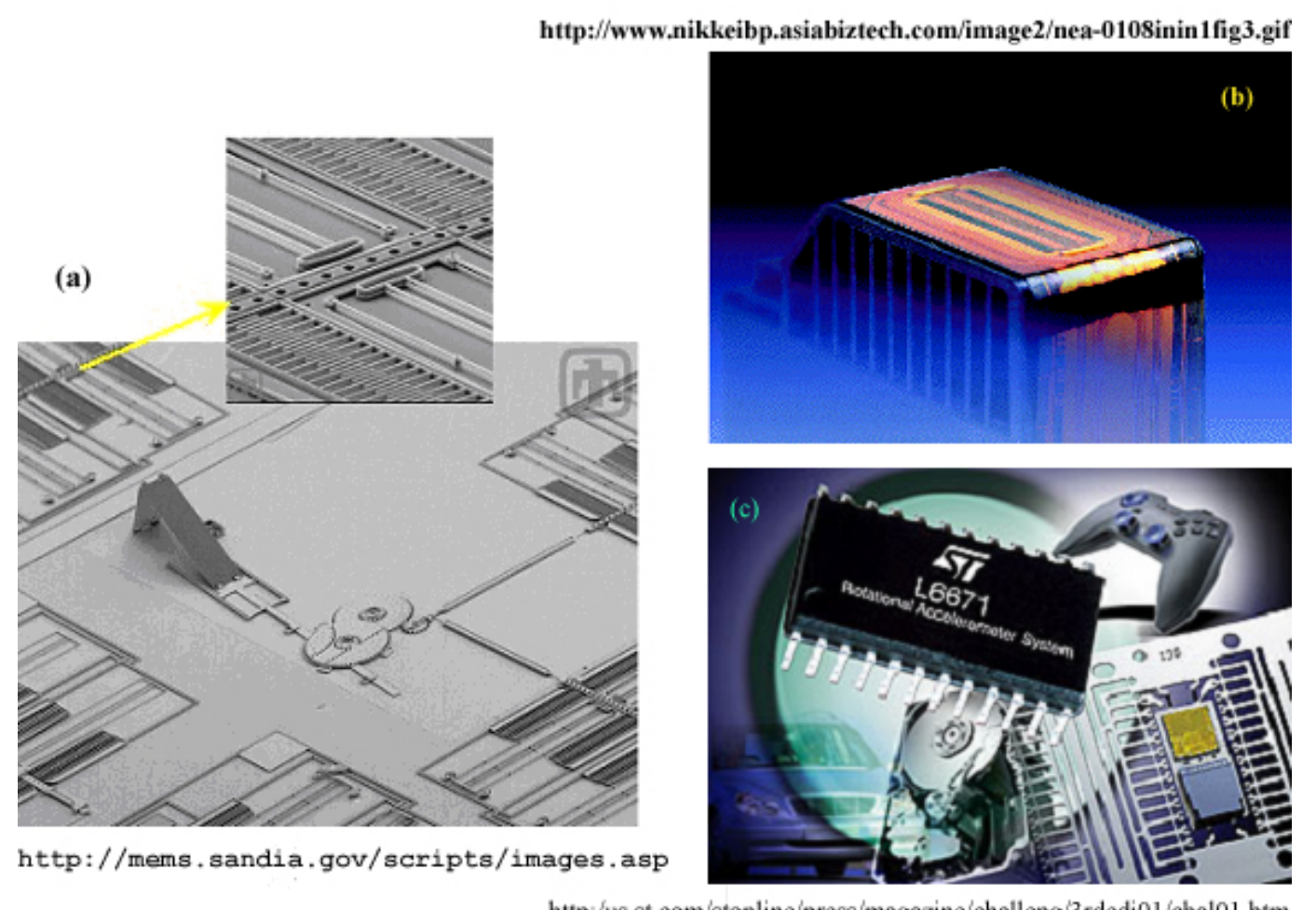

http:/us.st.com/stonline/press/magazine/challeng/3rdedi01/chal01,htm

Figure 1-1: MEMS examples: a) Sandia's silicon mirror and its comb drive system, b) Inkjet printer head, c) STMicroelectronics' rotational accelerometer 
It has been said that the MEMS industry is now in the same state as the semiconductor industry was in 40 years ago [6]. It is also believed that MEMS is the next logical step in the silicon revolution. MEMS products were first developed in the 1960s as accurate hydraulic pressure sensors for aircraft. The first commercial MEMS product was sold in 1993, which was Analog Devices' surface micromachined accelerometer (Figure 1-2). Before 1987, MEMS structures were limited in motion. Techniques for integrated fabrication of mechanisms (i.e. rigid bodies connected by joints for transmitting, controlling, or constraining relative movement) on silicon were demonstrated during 1987-1988. Today, MEMS has the ability to integrate microengines, microcircuits, microsensors, and microactuators into the silicon base, which allows MEMS not only to collect, process and send message or commands, but also to automatically take action in response to external commands/instructions.

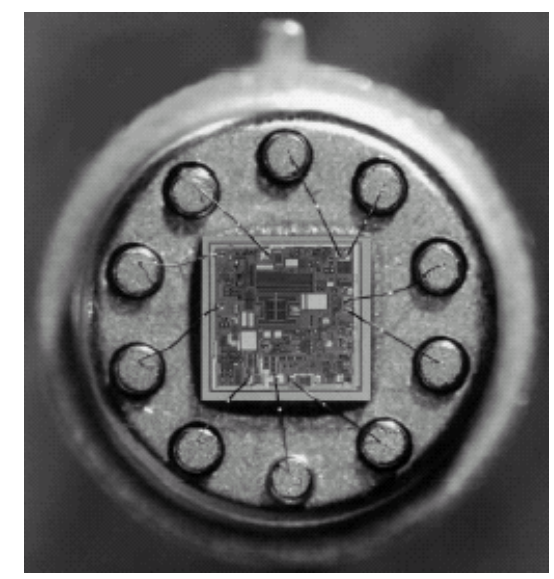

Figure 1-2 Analog Devices’ Si accelerometer based on hybrid technology (ADXL-50) (www2.ncsu.edu/.../ F98-S12/memsoverview.html)

\subsection{Current Trends in MEMS Technology}

\subsubsection{MEMS Application}

Currently, MEMS technologies are mainly used in the following applications.

\section{- Microsensors}

Microsensors are the most developed and widely used MEMS devices. The first successful commercial microsensors are acceleration microsensors (accelerometers) used 
for airbags in automobiles, and these devices quickly replaced conventional accelerometers. Other prominent microsensors allow the measurement of force, pressure, position, speed, chemicals, biological agents, and temperature.

\section{- Microactuators}

The typical microactuators are micromotors that, based on their motion mode, can be categorized as rotational or linear. Other microactuators include switches, oscillators, valves, and pumps. Some amazing effects can be achieved by arranging microactuators into arrays, such as the positioning and transportation of very small objects. Although many microactuators are driven by electrostatic force, devices now can also be driven by piezoelectric forces, magnetic forces, shape memory alloys, as well as thermal and thermal phase change forces.

\section{- Micro-Opto-Electro-Mechanical System (MOEMS)}

MOEM devices and systems employ MEMS to sense, detect, or manipulate light to affect light intensity or phase modulation, beam steering, and beam shaping and conditioning based on refraction, reflection, or diffraction principles. With the development of information technology and broadband optical communications, MEMS and MOEMS devices become more and more important. MOEMS technology can be used in optical communication devices (switches, cross-connect matrix, DWDM systems etc.), digital image processing, and adaptive optics. Furthermore, MOEMS also makes contributions to the industrial maintenance, environmental and medical industries.

Figure 1-3 is a sample of packaged MOEMS device from InterScience. The standard DIP packaging increases the flexibility of MOEMS device to interface with other devices.

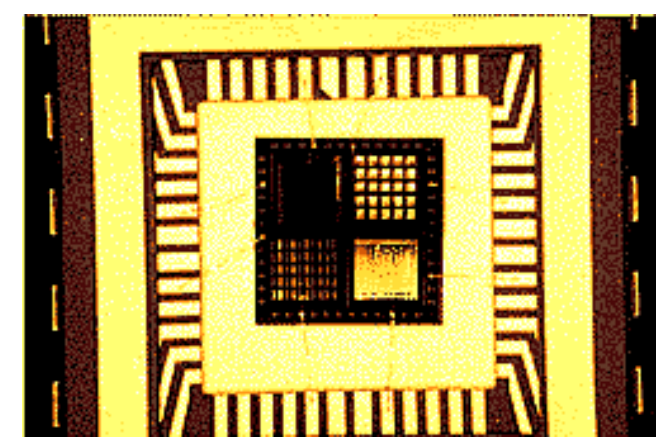

Figure 1-3: Close-Up of a MOEMS Die (http://www.esiee.fr/ francaio/filieremems/filiere.html) 


\section{- Micro Biochemical Devices}

The medical market is incredibly attractive for MEMS. MEMS technology has already provided dramatic and tangible improvements in both patient healthcare and outcomes. Even more exciting are the devices under development that will soon come to market. Figure 1-4 is an example of a pressure sensor, which is designed by ISSYS, and can be inserted into a diagnostic catheter.

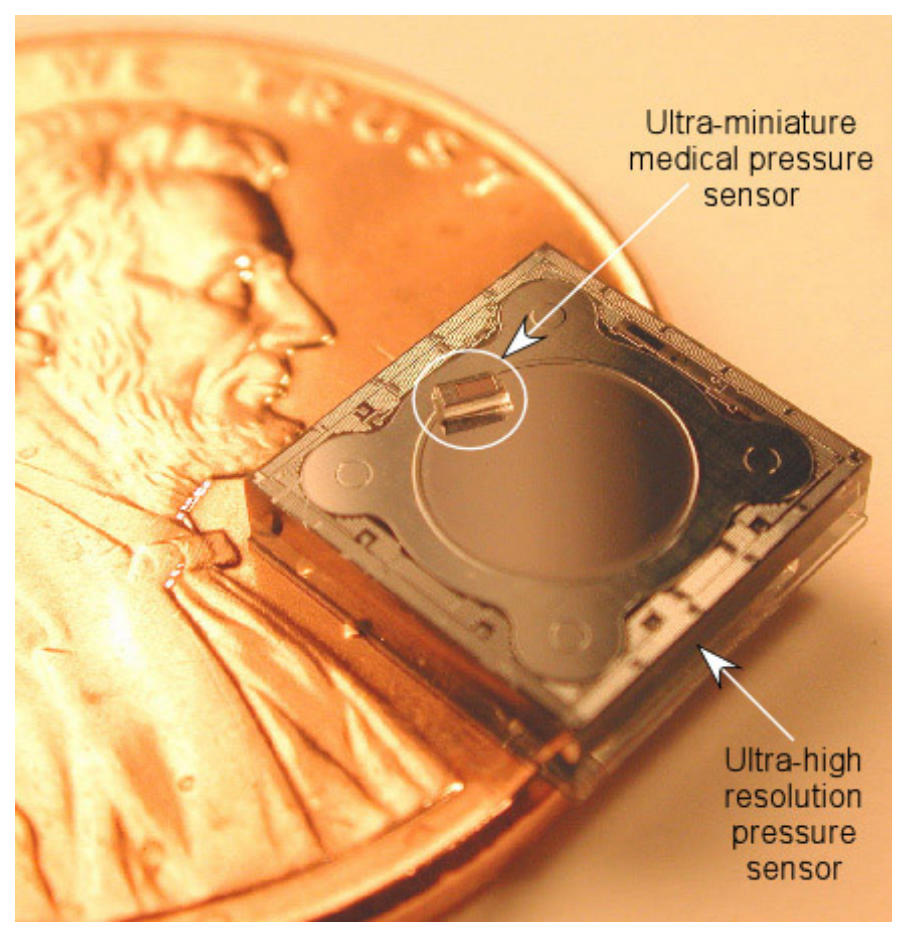

Figure 1-4: ISSYS sensor on a penny (http://www.mems-issys.com/html/products-services.html)

\subsubsection{MEMS Fabrication}

Although many of the micro fabrication techniques used for the IC industry can be used to process MEMS, some micro fabrication processes have been developed solely for MEMS to meet the unique 3D fabrication requirement for micromachines, and the requirement for integrating multiple patterns to form a complete MEMS device.

\subsubsection{IC Fabrication:}

Knowledge of basic IC fabrication technology or microfabrication is very helpful for the understanding of MEMS fabrication. The conventional IC processing includes the following steps [7]: 
- Oxidation: silicon dioxide film can be formed by oxidizing the surface of the substrate, which is a high-quality electrical insulator, and can be used as a barrier material

- Photolithography: during this process, mask patterns are transferred to the surface of the substrate

- Etching: this process is used to remove any barrier material not protected by photoresist

- Diffusion: this process is to deposit high density concentration of desired impurity on the surface of the substrate, which is the primary method to introduce impurities to control the majority-carrier type and resistivity of layers formed in wafer.

- Evaporation or sputtering: this process is used to deposit insulators or metal films on substrate materials

- Chemical vapor deposition (CVD): this process is used to deposit thin films of metal, silicon nitride, silicon dioxide, and polysilicon

- Ion implantation: this process is an alternative to diffusion

\subsubsection{MEMS Fabrication:}

Based on these well-developed IC techniques, additional processes have been developed for fabrication of MEMS. The processing of MEMS can be sub-grouped into: bulk micromachining, surface micromachining, bonding, nonsilicon microfabrication and integration of circuits.

Both bulk micromachining and surface micromaching date back to the 1960's, and were developed in parallel. Although some process steps are common for both techniques, there are notable differences. Combinations of the two techniques also exist. Both bulk micromachining and surface micromaching are commonly used for silicon, while bulk micromachining is the predominant technique used for quartz.

\section{Bulk Micromachining:}

Bulk micromachining is based on the IC technology for the fabrication of 3D structures. It is a process of etching silicon wafers or other substrates to produce features directly onto it. Bulk Micromachining uses both wet and dry etching techniques to sculpt 
high-precision complex three-dimensional shapes from the $\mathrm{Si}$ substrate, such as $\mathrm{V}$ grooves, channels, pyramidal pits, membranes, vias and nozzles [8].

\section{Surface Micromachining \& MUMPs(Multi-User MEMS Processes):}

Surface micromachining enables the fabrication of complex multicomponent integrated micromechanical structures, which would not be possible in traditional bulk micromachining. This technique deposits patterned layers of sacrificial and structural material on the surface of a silicon wafer. When the sacrificial material is removed, completely formed and assembled mechanical devices are left. The most widely used surface micromachining technique, polysilicon surface micromachining, uses $\mathrm{SiO}_{2}$ as the sacrificial material and polysilicon as the structural material.

The substrate wafer is used primarily as a mechanical support on which multiple alternating layers of structural and sacrificial material are deposited and patterned to form micromechanical structures. The sacrificial material is then dissolved in a chemical etchant, which does not affect the structural parts. Figure 1-5 shows the process of making a beam. With this technique, Texas Instruments has built a large array of mirrors for projection TVs by depositing and patterning aluminum over a sacrificial polymer layer.

The MEMS devices involved in this research were fabricated by a commercial program called MUMPs (Multi-User MEMS Processes), which is developed by Cronos, a division of JDS Uniphase (now MEMSCAP). MUMPs provides “cost-effective, proof-ofconcept MEMS fabrication to industry, universities, and government worldwide” [9]. With different process techniques, MUMPs can be sub-grouped into three categories:

- PolyMUMPs: a three-layer polysilicon surface micromachining process

- MetalMUMPs: a electroplated nickel process

- SOIMUMPs: a silicon-oninsulator micromachining process.

The PolyMUMPs process utilized in this research is a three-layer polysilicon surface micromachining process initiated in the late 80's and early 90's by Berkeley Sensors and Actuators Center (BSAC) at the University of California. PolyMUMPs has the general features of standard surface micromachining process with the ability of 
supporting different designs on a single silicon wafer, and is designed to be as general as possible to meet the requirements of a variety of users.

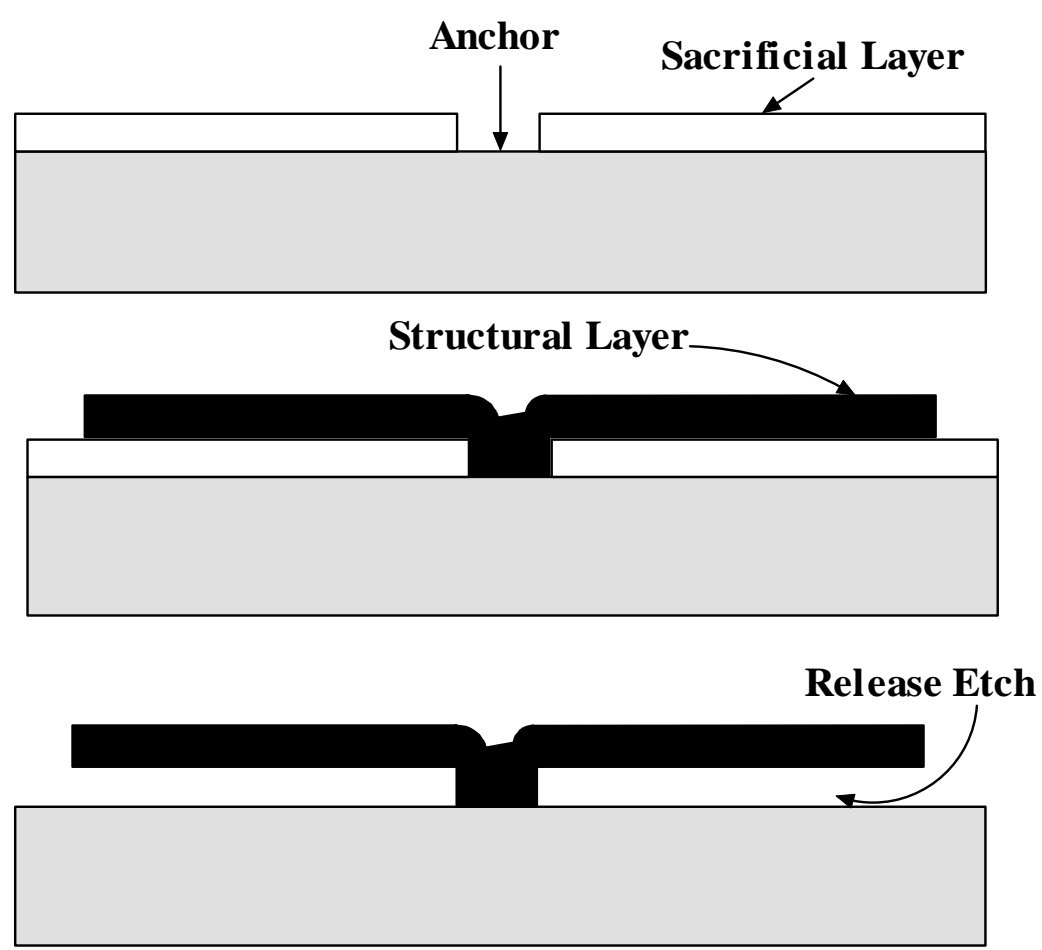

Figure 1-5 Surface Micromachining

\section{LIGA (LIthographie, Galvanoformung, Abformung)}

LIGA is increasingly demonstrated to be a viable technology in the microfabrication of MEMS devices. The LIGA process exposes PMMA (polymethyl methacrylate) plastic to synchrotron radiation through a mask. Exposed PMMA is then washed away, leaving vertical wall structures with spectacular accuracy. Metal is then plated onto the structure, replacing the PMMA that was washed away. This metal piece can become the final part, or can be used as an injection mold for parts made out of a variety of plastics.

\section{Bonding}

Silicon wafer bonding has become the cornerstone of the MEMS manufacturing technology. It is often done on a wafer level, and can be used to create cavities, to 
package the sensor chips, etc. There are many kinds of bonding processes such as integrated LPCVD nitride bonding process, localized eutectic bonding, fusion and solder bonding process, RTP (rapid thermal processing) bonding process, nanosecond laser welding process, ultrasonic sealing process, localized CVD sealing process and lowtemperature solder bonding process.

\subsubsection{MEMS Failure and Reliability}

Despite the benefits that MEMS offers, only a few applications (such as ink jet heads, projection displays, pressure sensors, and accelerometers) have been successfully commercialized. There are still a number of fundamental challenges for MEMS to have the same prevalence and manufacturability as the integrated circuit. These challenges include the following:

a) Advanced simulation and modeling tools for MEMS design are required, because currently most MEMS devices are modeled by weak analytical tools which directly result in a relatively inaccurate prediction of performance.

b) Packaging of MEMS devices and systems needs to be improved considerably.

c) In order to separate the design and fabrication of MEMS, an interface between them is required.

d) Quality control standards for MEMS technologies are required [10].

e) MEMS reliability issues should be solved.

The quality of many MEMS devices fabricated at either academic or commercial facilities is relatively low. This is partially because the technology is so new that the fabricators do not yet know how to measure the quality, let alone how to improve it. This causes the reliability of many MEMS devices to be too poor to be accepted. The reliability of MEMS devices is the most critical criterion for determining suitability for commercialization. A good understanding of how MEMS devices fail is very important in the effort to improve their reliability.

\subsubsection{MEMS Failure}

Thin film polycrystalline silicon is one of the most common materials used in MEMS. The mechanical properties of this brittle material are critical to reliability and 
performance of MEMS devices. Currently basic mechanical properties of thin films, such as Young's modulus, Poisson's ratio, and tensile strength, cannot be completely characterized, and the applicability of standard test techniques and specimen geometries remains undetermined.

The primary causes of MEMS failure are fatigue, creep failure, stiction, wear, delamination, environment-induced failures, mechanical instability, and electrical instability caused by Na contamination (the ion mobile in the presence of electric fields). Improper operation methods can also significantly degrade performance of MEMS [11].

Fatigue is a failure mechanism caused by periodic force that acts on a device and is below the yield or fracture stress of the material. This load leads to the formation of surface microcracks that cause the slow weakening of the material over time and create localized plastic deformations. Fatigue also causes a gradual change in the properties of a material. After repeated cycling, Young's modulus will gradually shift, which will lead to a change in resonant frequency. The dampening coefficient is also affected, which will increase over time and change the resonant frequency and Q factor of a structure [12]. Electrical resistance of many structures will also increase over time. The combined effects of these changes can lead to degradation and failure.

Creep is the time-dependent plastic deformation of materials at high temperatures. Many materials, including metals, exhibit creep at temperatures above half of the melting point of the material [13]. Fractures in thin films are normally constrained by the substrates. This constraint will be lost over time if the substrate creeps, which can result in thin films cracking, and eventually affecting the reliability of MEMS devices.

Stiction is recognized as a major potential failure mechanism in surface micromachined MEMS. When two polished surfaces come into contact, they tend to adhere to each other due to adhesive forces, such as capillary and electrostatic force, resulting in impaired functionality or even failure of MEMS devices.

Wear is defined as the removal of material from a solid surface resulting from the mechanical action [14] caused by the motion of one surface over another. Wear is generally considered an undesirable effect on MEMS because it can also result in impaired functionality and mass changes. There are mainly four types of wear due to different causes: 
- Adhesive wear occurs because of one surface sliding over another. During the sliding, the capillary or sometimes electrostatic force will bond two materials together. When the bonds break, they are unlikely to separate at the original interface, causing fractures in one of the materials.

- Abrasive wear occurs when a hard, rough surface slides over a softer one and strips away underlying material.

- Corrosive wear occurs when two surfaces chemically interact with each other and the sliding process strips away one of the reaction products.

- Surface fatigue wear occurs mostly in rolling applications, such as bearings and gears. In this case, rolling instead of sliding damages the polished surfaces. Over time, the continued stressing and unstressing of the material under the roller will cause the appearance of fatigue cracks. These cracks then propagate parallel to the surface of a structure, causing material to flake off the surface.

Delamination happens when the interface of two materials loses its adhesive bond. The result of delamination for a MEMS device is a catastrophic failure. If the material is still present on the device, it can cause shorting or mechanical impedance. The loss of mass will alter the mechanical characteristics of a structure, depending on the amount of material that has fallen off. With delamination is the cause, up to twenty five percent resonant frequency shifts has been reported in some devices [15].

Stray stresses are stresses in films when there is no external force present. For MEMS, small stresses can cause noise in sensor outputs and large stresses can lead to mechanical deformation.

Parasitic capacitance is another failure mechanism in MEMS, since parasitic capacitance can cause unwanted electrical and mechanical behavior in devices.

Environmentally Induced Failure Mechanisms, in addition to device operation, there are external effects that can also cause failure in MEMS. Many environmental factors can lead to failures.

- Vibration is a big reliability concern in MEMS. External unwanted vibrations can introduce failure, either through inducing surface adhesion or through fracturing support structures of devices. Furthermore, long-term vibrations also contribute to fatigue. 
- Shock differs from vibration in that shock is a single mechanical impact instead of a rhythmic event. Shock creates a direct transfer of mechanical energy across the device, which can lead to both adhesion and fracture. Shock can also cause wire bond shearing, a common package failure mode for all semiconductor devices.

- Humidity is another major factor in MEMS failure [16]. Surface micromachined devices are extremely hydrophilic. When devices are studied in a humid environment, water can condense on structure surfaces. It has been experimentally proven that, for a surface micromachined device, the residual stress in the structure can be increased by humidity [17].

- Radiation Effects: It has long been known that electrical systems are susceptible to radiation. Recent research has shown that mechanical devices may also be sensitive to radiation-induced damage, especially for those devices that have mechanical motion governed by electric fields across insulators, such as electrostatically positioned cantilever beams.

- Particulates are fine particles, which are prevalent in the atmosphere. These particles can electrically short out MEMS and can also induce stiction. Particulates present during the fabrication process can also cause problems with adhesion. When dust particles are present, the bond between materials will weaken, and this will likely cause delamination problems.

- Temperature change is a serious concern for MEMS since internal stresses in devices are extremely temperature dependent. Besides, thermal effects may cause problems in metal packaging. The thermal coefficient of the expansion of metals can be ten times greater than that of silicon.

- Electrostatic discharge (ESD) is known to have fatal effects on circuits and could have similar effects on MEMS.

Mechanical Instabilities: Microactuators are typically driven by electrical force such as capacitive attraction. Undesired forces can result in mechanical instability, causing both performance degradation and premature failure. For example, for the lateral comb drive actuator, relatively large electrostatic forces are acting on the comb fingers in the lateral direction. Usually these forces can be eliminated as long as the shuttle is 
perfectly aligned. However, with even slight perturbations, these forces will become very asymmetric, leading to the buckling of the support springs.

Operation Methods: Improper operation methods can significantly degrade performance and reliability of MEMS. MEMS actuators are usually designed as physically constrained systems. All the forces associated with the system balance result in the dynamical behavior of the system. If appropriate drive signals are not provided, significant constraint forces can result in compensating for other forces, such as inertia. Constraint forces, particularly those utilized to operate MEMS in a way that minimizes forces between rubbing surfaces, are very important. Employing improper drive signal for the MEMS device can result in significant difference in operation. Experiments show that MEMS actuators driven by model-based drive signals have 5 orders of magnitude longer life than those driven by square wave signals.

Manufactory imprecision: Along with all of the reliability issues listed above, manufacturing tolerance also contributes to the performance decrease of the MEMS devices. A tiny size imprecision of a device could lead to a huge shift of device characteristics. Figure 1-6 shows the possible difference for a lateral comb resonator device with the same design and manufacturing steps.
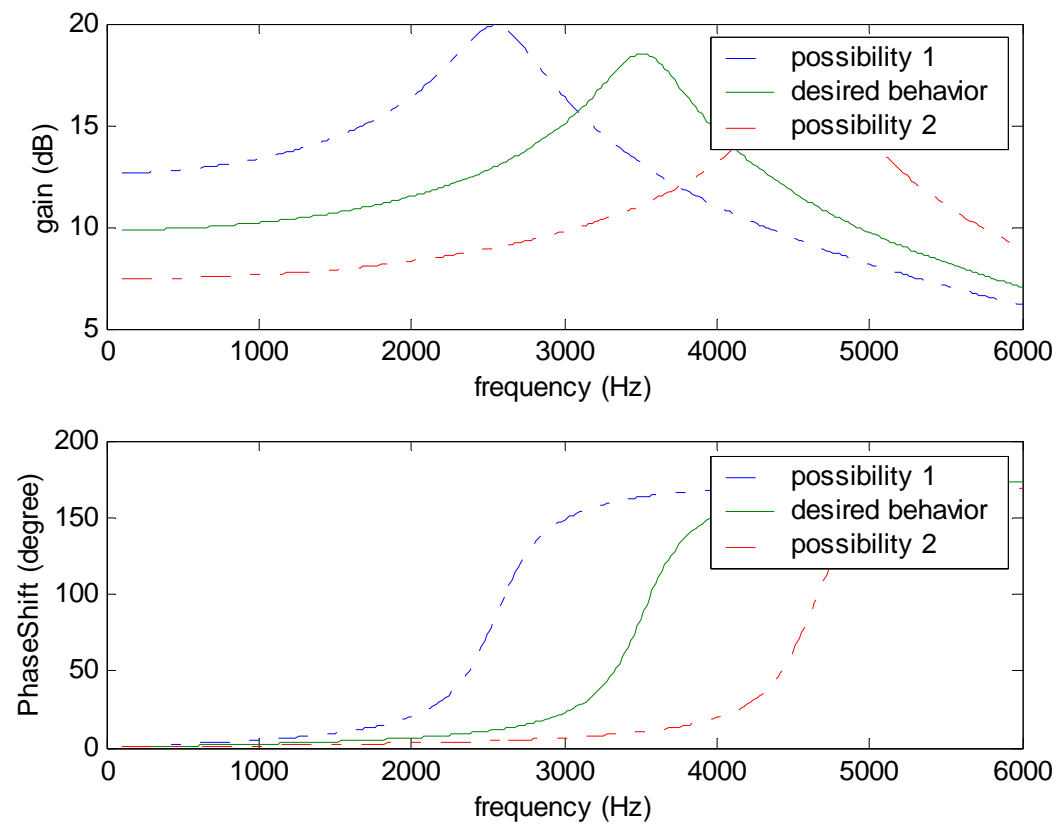

Figure 1-6: Bode plot of a microactuator, (solid - desired behavior; dash line - possible behavior of this device within manufacturing tolerance) 
In order to improve the reliability and to minimize device losses, significant research has been performed to investigate device failure [18]. Using closed-loop control methods to avoid the failure that causes device characteristics shifts is the most promising way to increase MEMS reliability.

\subsection{MEMS Control}

Although process control is important to the development of MEMS devices, applying control schemes to MEMS itself has lagged in development, compared to other areas of MEMS development such as fabrication and structure design. To date, except for a few successes in vertical motion control, most MEMS control efforts are in simulation or theoretical analysis stages [18-28].

Vertical Motion Control: Shekhar Bahansali et al have successfully developed a one degree-of freedom microsuspension system, which has a built in active position control unit as a paradigm of micromagnetic bearings [19]. He declared that the vertical position (Z-direction) of the magnet and flexible beam structure could be elevated and maintained at a predetermined level by a PD control system.

Success of feedforward control for a dual stage servo system in disk drives has also been reported [20]. A decoupled controller architecture and discrete time pole placement design methodology has been employed. A feed forward compensator and PI controller have been developed for the closed-loop feedback control of micromirrors for fiber optical switching [21]. Chen et al performed model analysis on an unbalanced comb resonator which is used to actuate the nanomirrors used in EUV maskless lithography [22]. The suggested model is nonlinear, and open-loop operation has been performed, however, no signal observing method has been mentioned for this work.

Magnetic force-driven actuator: Positive experimental results also have been achieved for magnetic force-driven microactuators [23]. In this work, both Lyapunov stability theory and admissibility framework have been applied in parallel to design and analyze a non-linear controller for MEMS actuators. No details regarding the experiment or signal measurement method have been addressed. 
Other types of motion control: For a cantilever-based mass sensor, the change of mass added to the cantilever produces no change in the elastic constant, only a shift in its resonant frequency. Therefore, for this kind of mass sensor, the minimum change of mass that can be detected depends on the minimum change in resonant frequency. This, in turn, depends on the quality (Q) factor of the cantilever, since a low Q-factor implies a small variation of amplitude with frequency. That indicates the resolution of this kind of mass sensor can be increased by improving the cantilever's Q-factor. With an optical detection system, G. Abadal et al have experimentally found that the cantilever behaves as a $2^{\text {nd }}$ order system [24]. Based on this result, they performed a simulation by adding an additional electronic feedback using the Q-factor as the feedback signal. The Q-factor can be increased enough to achieve the maximum sensitivity, even in a liquid environment where the Q-factor is expected to be very low.

When applied voltages are increased beyond a certain critical voltage, there is no longer a steady-state configuration for many electrostatically actuated devices where mechanical members remain separate. This is known as pull-in voltage instability. Seeger et al performed theoretical analysis and simulation of feedback control of charge on a gap-closing actuator to act against voltage pull-in [25]. They found that, when the parallel parasitic capacitance is sufficiently small, and when the device operates on a voltage slightly higher than the structure pull-in voltage, a properly designed switched-capacitor charge-control circuit can stabilize a gap-closing actuator against voltage pull-in. Pelesko and Trolo, also performed theoretical analysis for the control of pull-in voltage instability [26]. Their result shows only the local problem that caused pull-in voltage instability could be solved, not the non-local problem.

Garcia and Sniegowski have proposed a simple square wave-driven open-loop method to control a microengine to provide torque to drive micromechanisms [27]. In their simulation result, ripples in the control torque and relevant speed are significant. To improve the system performance, a dynamic operation model has been proposed [28], which is also an open loop method. Lyshevski has developed a system model considering the non-linearity of a microengine[29], but no experimental or simulation results have been reported. 


\subsection{Integrated Through Wafer Monitoring Method}

Compared with macro-scale feedback control applications, new challenges are faced when considering in-situ MEMS control due to their small size. It is necessary to have a microstructure monitoring method for acquiring an accurate position signal with which to implement feedback control. Since regular sensors cannot be used to detect the small range of MEMS output, a proper monitoring method suitable for micron-scale application must be developed. MEMS time domain monitoring methods can be divided into two categories: 1) real-time methods and 2) time-averaged techniques. Even though the latter one can measure motions at nanometer resolution and at frequencies up to $5 \mathrm{MHz}[30,31]$, this technique can be used only when the output is periodic or the transition is repeatable. The Laser Doppler Vibrometer (LDV) is one of the most popular monitoring techniques in the real-time category. The key feature of LDV is that it measures real-time transient response under typical operating conditions where both electrical and mechanical effects are present. It is currently being used throughout the MEMS community to characterize the dynamics of micro-devices such as vibrating cantilevers, membranes, actuators, motors, micro-optics and torsional oscillators. However, the LDV technique is not perfect yet. Its main drawback is the inability to determine the phase relationship between measurements of different points [32]. Many monitoring schemes applied to MEMS use optical methods that provide low noise and high accuracy metrology, but these bulk optical systems are employed only during dielevel testing prior to device packaging [33]. Electrical means for sensing device motion during device operation have been explored [19], however, the dynamic range of the signal associated with device movement is small compared with the drive voltage signal. As a result, the signal may be hidden or masked in the noise created by the higher voltage used to drive the device [34].

A unique method has been developed to measure the output of a MEMS system in real-time. This method uses a through-wafer optical microprobe to detect the movement of a MEMS, which not only can measure the output signal in real-time but also decouple the observing signal from the driving signal $[35,36,37]$. The through-wafer optical position signal is produced by the interaction of the moving stage of the resonator with an infrared probe beam. The through-wafer optical microprobe setup used to study the 
feasibility of using such a signal to characterize and monitor device motion is illustrated in Figure 1-7. In this setup, the output of a pigtailed infrared (1310 nm) laser diode is focused through the die back to a spot size of 10-15 $\mu \mathrm{m}$ in diameter at the die top surface. After interaction with the grating structure fabricated in the resonator device shuttle, the transmitted intensity is coupled into an $8 \mu \mathrm{m}$ diameter single mode detector fiber, positioned 50-100 microns above the device die surface. This through-wafer optical method has been used in this study for monitoring the motion of the lateral comb resonator.
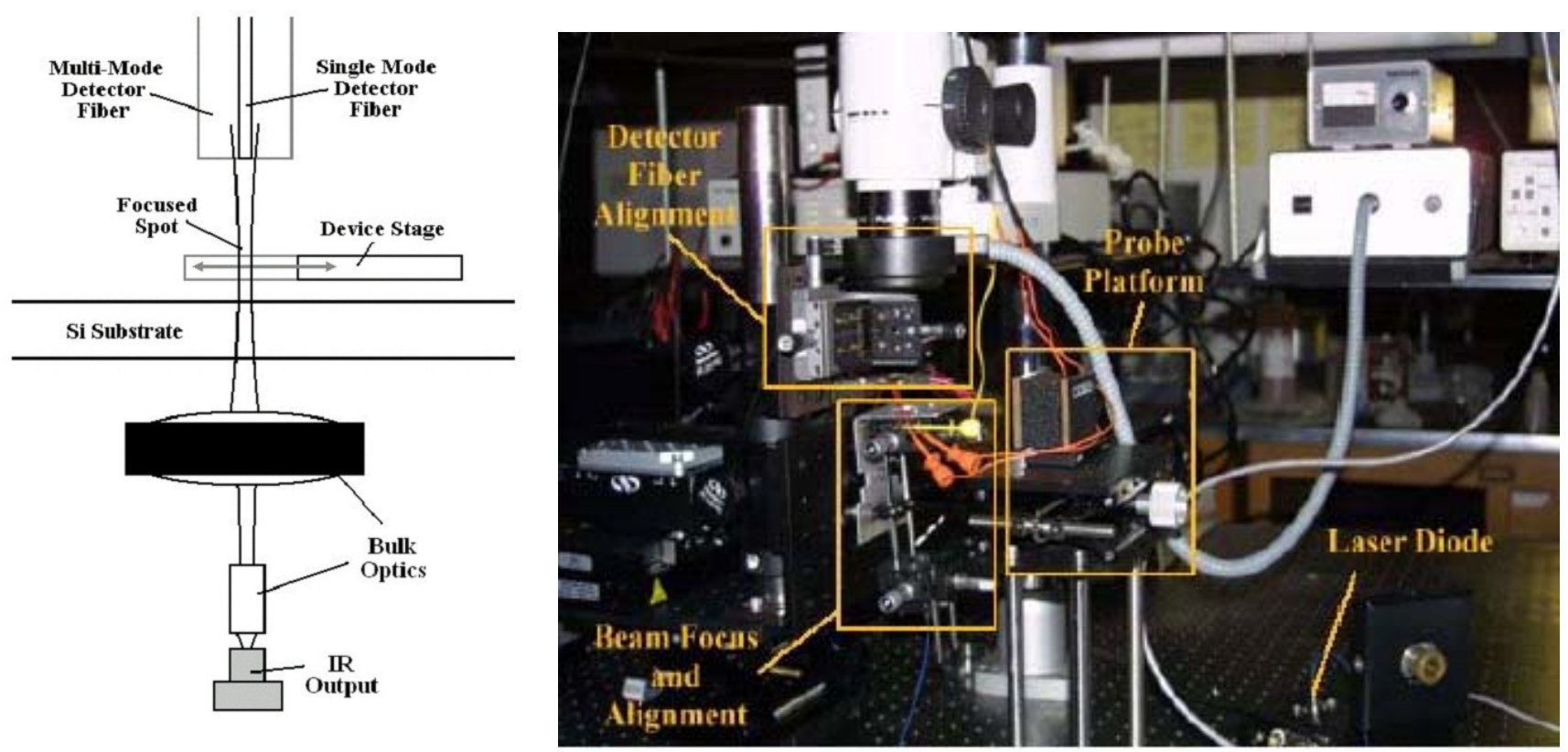

Figure 1-7: Experimental Setup of the Integrated Optical Monitoring

\subsection{Device Introduction (Single Opening \& Grating Structure)}

Microstructure Lateral Comb Resonators (LCR) that are designed by WVU and fabricated with MUMPS (Multi-User MEMS Processes) have been used in this study to explore the implementation of closed-loop control on a MEMS devices' lateral motion.

The mathematical model for the comb drive LCR can be described as [38]:

Equation 1-1

$$
F_{e}=m \ddot{x}+\beta \dot{x}+2 k_{s} x+F_{d},
$$


where:

$m$ : effective mass of the moving part,

$y$ : displacement of shuttle,

$k_{s}$ : spring constant,

$\beta$ : damping coefficient,

$F_{d}$ : load force, and

$F_{e}$ : electrostatic force (generated by the comb capacitor).

As shown in Figure 1-8, there are two different designs on the LCR's monitoring area, 'single opening' and 'grating structure'.

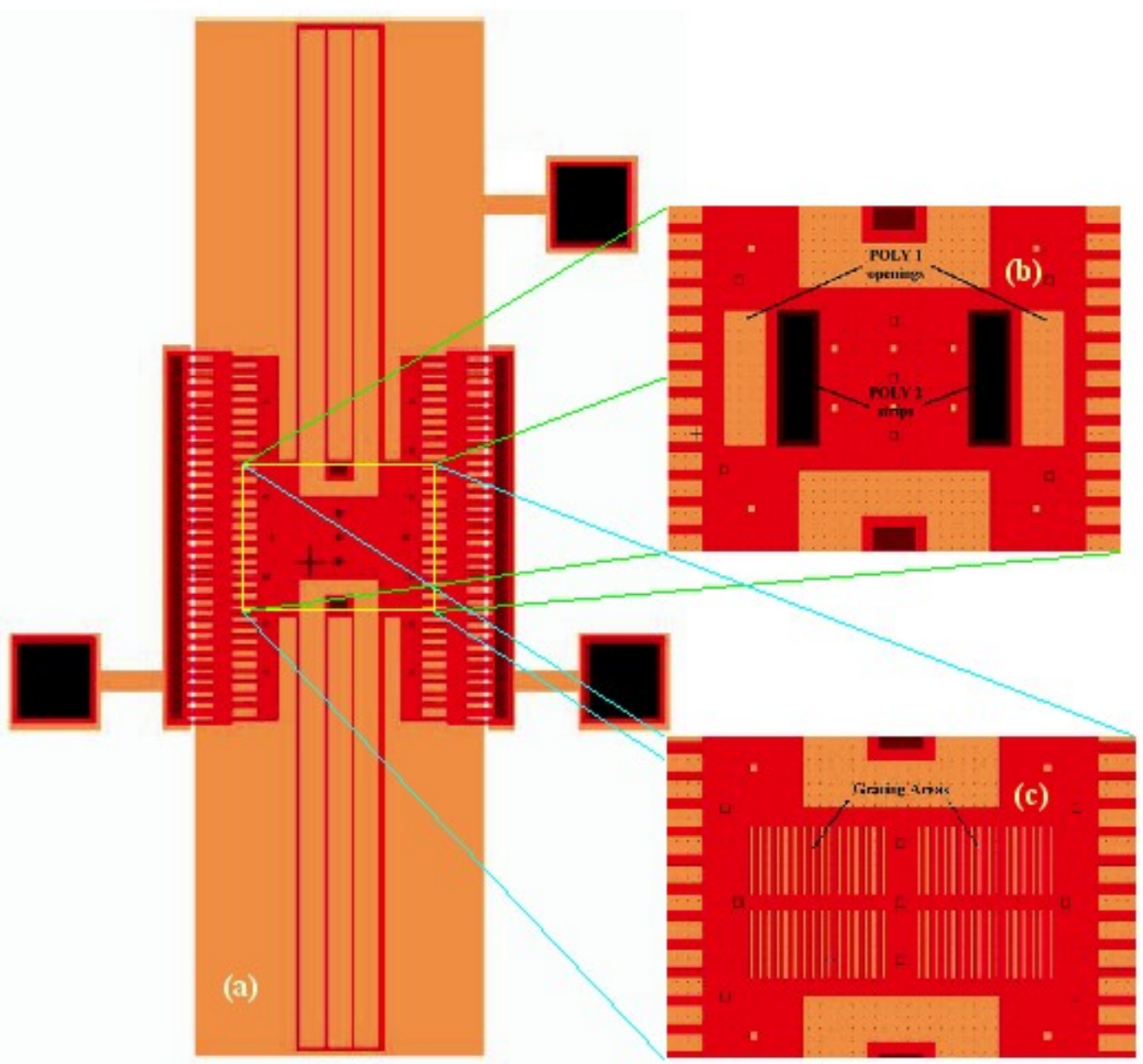

Figure 1-8: (a) basic LCR geometry, (b) single-opening through-wafer optical monitoring structure, and (c) grating-structure through-wafer optical monitoring structure. 
For the single opening device, the optical output is correlated with its shuttle movement (Figure 1-9). With this kind of observing signal, some closed-loop control effort can be applied to the LCR (Chapter 4). However, the absolute position of the shuttle cannot be determined based on the optical signal unless the ratio between the optical signal and the position signal is known. In other words, the accurate ratio of the optical signal to the shuttle is unknown. Furthermore, the ratio of (optical signal / position signal) is not a fixed constant, even an unobservable change on the alignment of the through wafer optical monitoring setup could change the ratio.

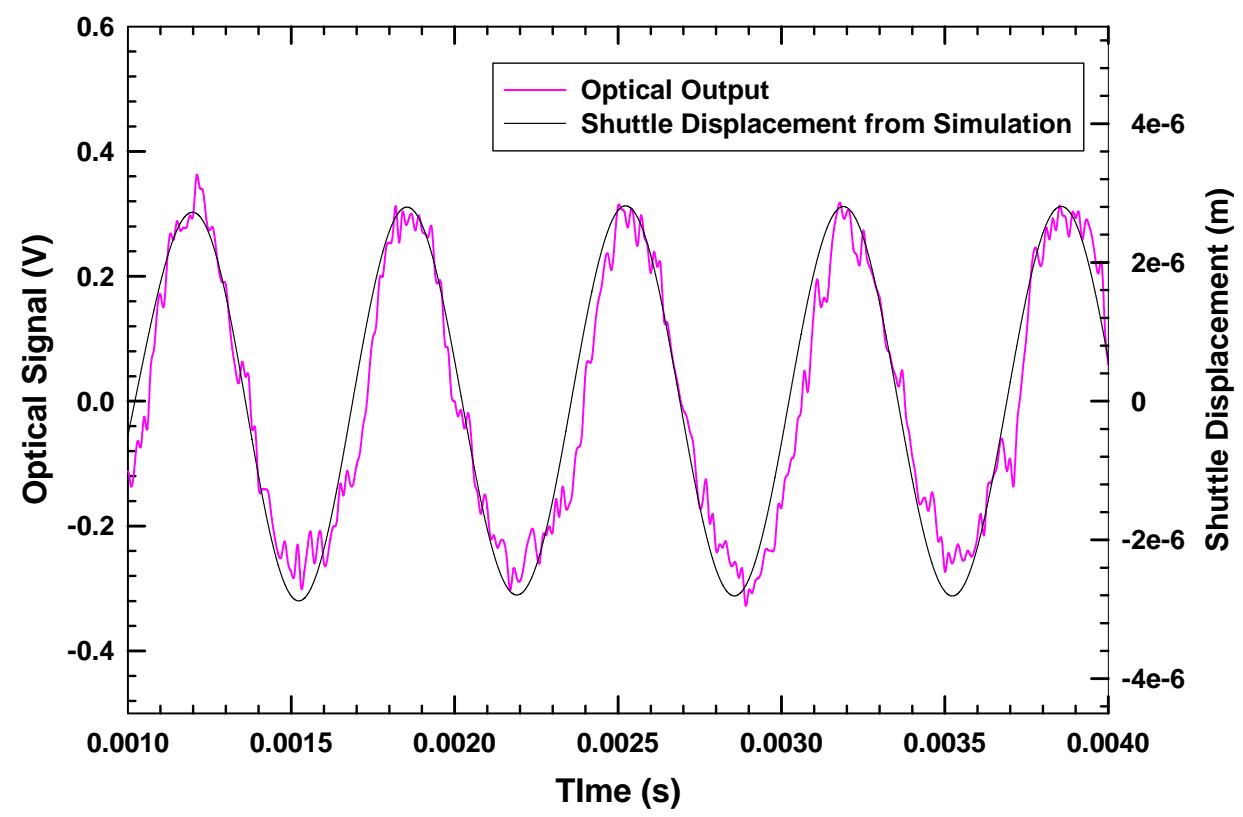

Figure 1-9: Relationship between Optical Monitoring Signal and Shuttle Displacement for the single opening LCR

For the grating structure device, the gratings ( $2 \mu \mathrm{m}$ slots with $2 \mu \mathrm{m}$ POLY1 separations) fabricated on the device translation stage provides absolute information for its corresponding position signal (Figure 1-10). With the grating structure, the optical output is no longer proportional to the position signal, $4 \mu \mathrm{m}$ 's stage travel causes two intensity maxima passing through the area of the detector, visually it can provide positional information at a precision of 2 micrometers.

For the grating structure LCR, the optical observation signal is no longer proportional to the shuttle's displacement. The grating $(2 \mu \mathrm{m})$ fabricated on the observing 
area will cause two intensity maximum changes when the shuttle travels 4 micrometers, visually it can provide displacement information with a precision of 2 micrometers.

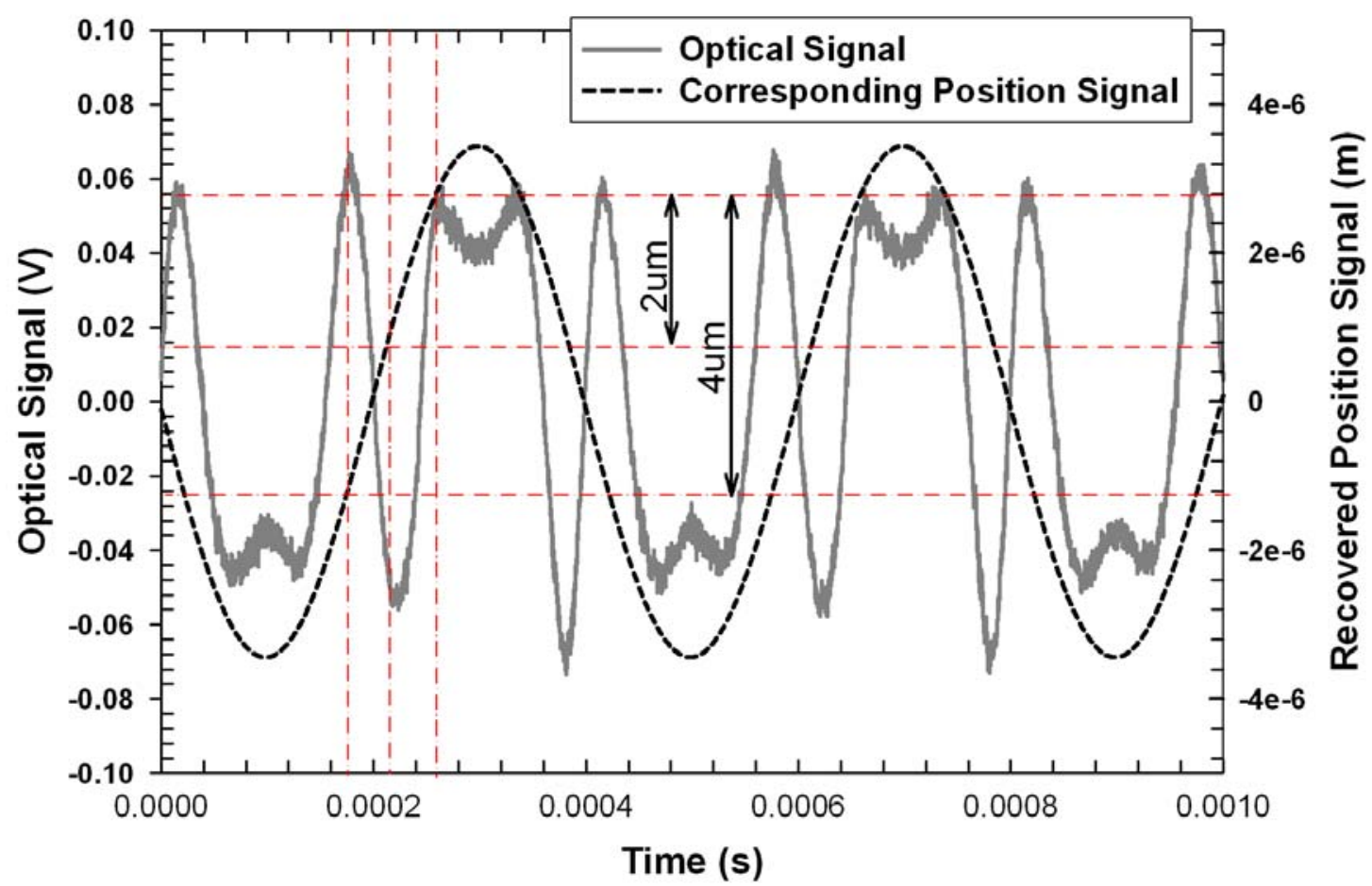

Figure 1-10: Relationship between Optical Monitoring Signal and Shuttle Displacement Signal for Grating Structure LCR 


\section{Chapter 2 Noise Analysis and Signal Filtering}

The detected through-wafer optical intensity (optical signal) is essential for development of system identification and implementation of feedback control. The noise in the optical signal is prominent (Figure 2-3) and noise reduction becomes an important issue in this study.

\subsection{Noise Analysis}

A comprehensive understanding of the corrupted optical signal is very important for implementing noise reduction on the optical signal. The lack of knowledge of signal to noise ratio makes the implementation of an effective noise reduction scheme very difficult.

\subsubsection{Optical Signal Analysis}

A sinusoidal voltage has been applied to the LCR, and the optical signal that represents the shuttle's movement has been recorded with an oscilloscope. The FFT spectrum of the optical signal shows that the dominant frequencies of the optical signal are harmonic of the force frequency. For example, when the frequency of force is $500 \mathrm{~Hz}$, the dominant components of the FFT spectrum for the corresponding optical signal are $500 \mathrm{~Hz}, 1000 \mathrm{~Hz}$, and $1500 \mathrm{~Hz}$... (Figure 2-1), and when the frequency of force is $700 \mathrm{~Hz}$, the dominant components become $700 \mathrm{~Hz}, 1400 \mathrm{~Hz}$, and $2100 \mathrm{~Hz}$... (Figure 2-2). Based on this observation, it can be assumed that the rest of the components in the optical signal could belong to the noise. A modified optical signal (Figure 2-3) has been generated based on this assumption. This signal is generated by applying inverse FFT on the improved power spectrum, which was obtained by keeping only the harmonic of $F_{\text {force }}$ (frequency of force) and setting the power magnitude of rest of the frequencies to zero. Where $k_{n}$ has been defined as: the power magnitude at $k_{n} \cdot F_{\text {force }}$ is about $50 \%$ of the average power magnitude of the noise. Comparing with power magnitude of this "clear" signal, the power magnitude of the noise is almost negligible (Figure 2-1, and Figure 2-2), which means the overlap between the spectrum of signal and the spectrum of noise is small. 


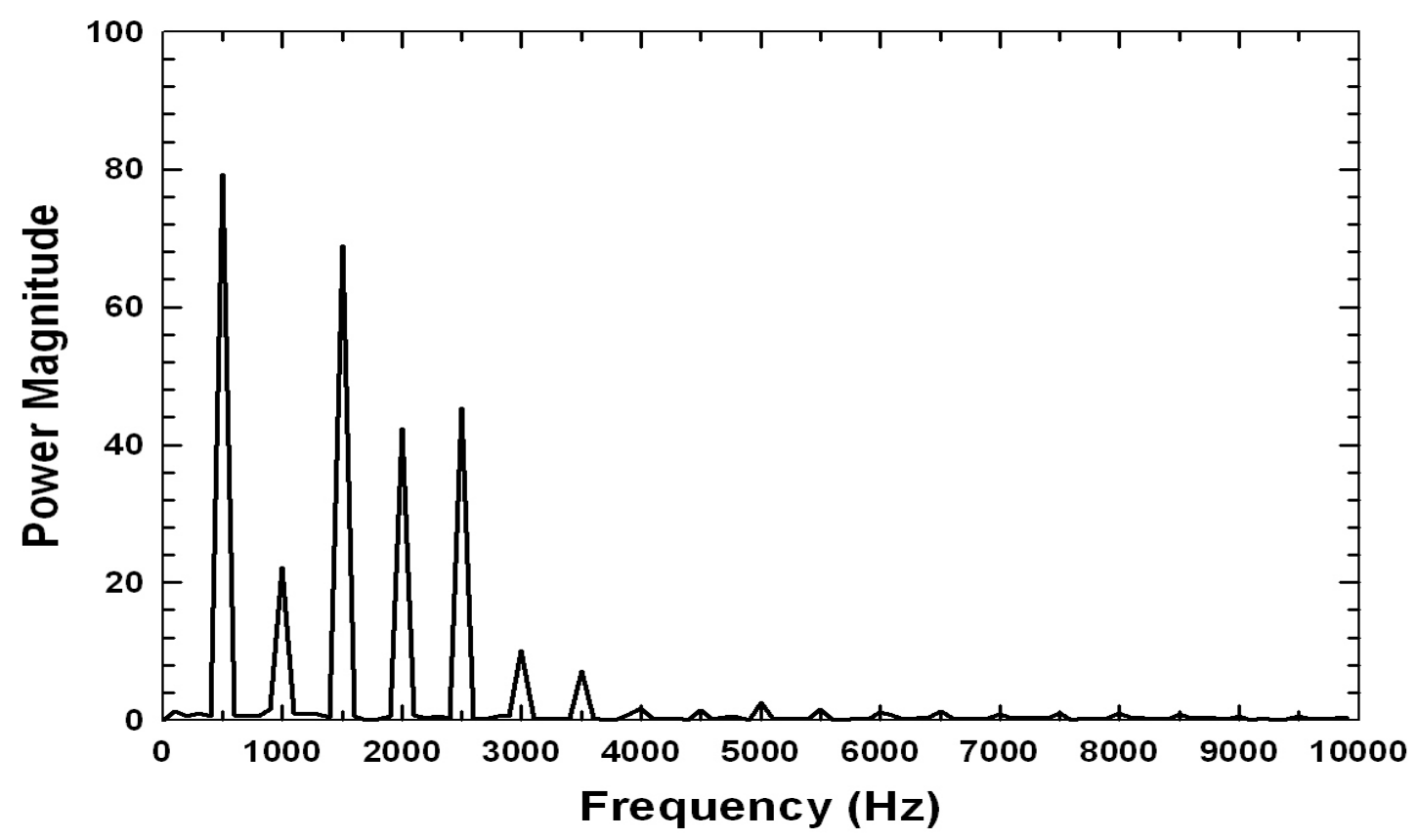

Figure 2-1: FFT Spectrum of Optical Signal While the Frequency Applied is $500 \mathrm{~Hz}$

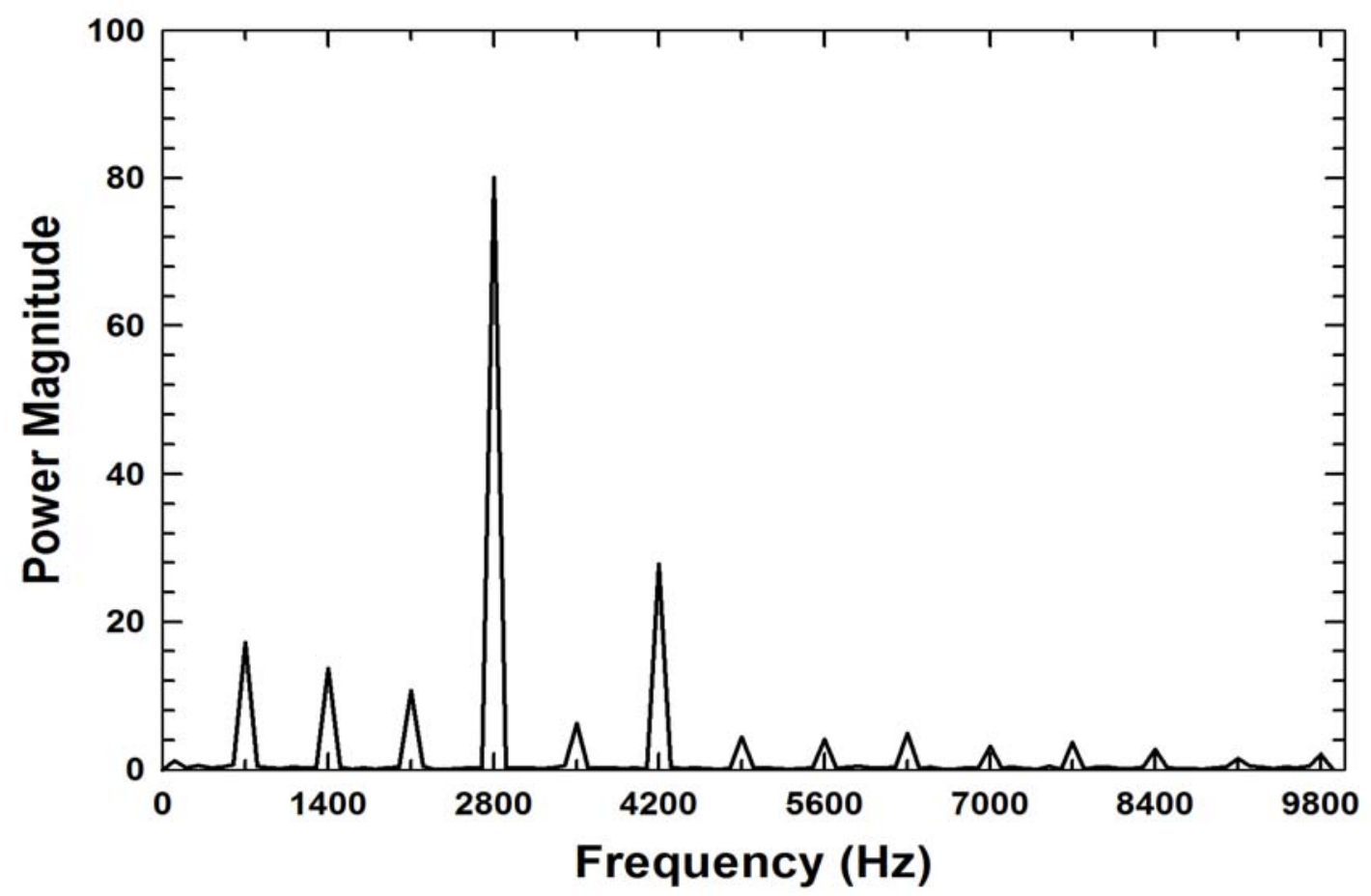

Figure 2-2: FFT Spectrum of Optical Signal While the Frequency Applied is $700 \mathrm{~Hz}$ 


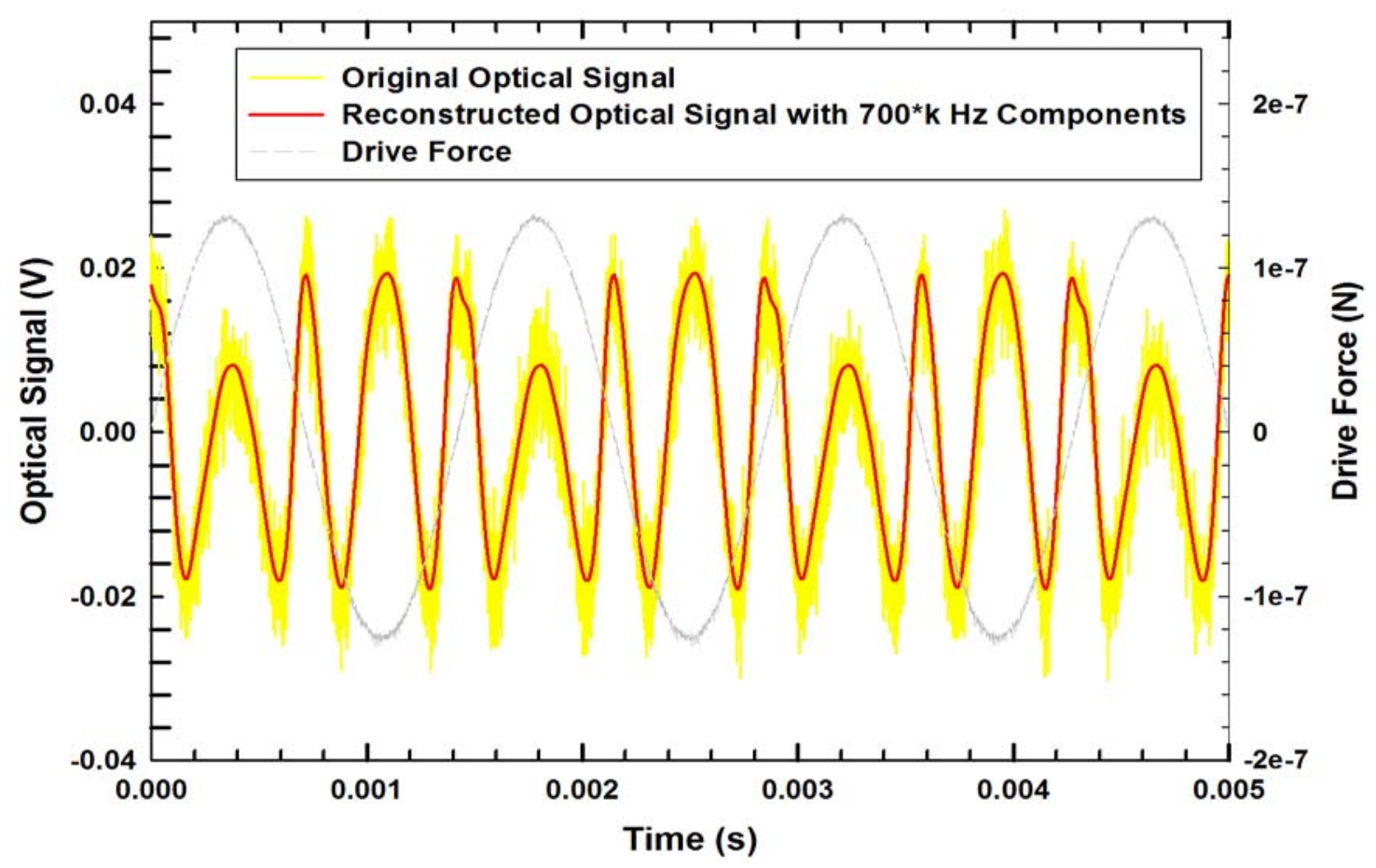

Figure 2-3: Original and Reconstructed Optical Signal with Harmonic Components

The difference between the original optical signal and the reconstructed signal has been considered as removed noise. To avoid removal of useful information from the optical signal, more analysis on the removed noise signal has been performed.

Two pieces of noise signals, fns1 and fns2 (Figure 2-4 (a), (b)), have been generated by subtracting clear signals from the original optical signals which were recorded by oscilloscope using the driving frequency of $400 \mathrm{~Hz}$ and $700 \mathrm{~Hz}$ respectively. The FFT spectra of these noise signals have been examined (Figure 2-8 (a), (b)). There is no dominant frequency within the FFT spectra. The noise signals are approximately white. However, the observation that the noise signals are random does not necessarily imply that all random signals are noise. Therefore, the possibility that some information has been overdrawn from the optical signal still exists.

Two pieces of optical signals, pns1 and pns2 (Figure 2-4 (c), (d)), of the optical through wafer setup itself have been recorded. Since these two signals are totally independent of the LCR's output, they can be treated as pure noise in this study. The two 
pieces of noise signals, pns1 and pns2, and the two removed noise signals, fns1 and fns2, have been carefully compared in both time domain and frequency domain.
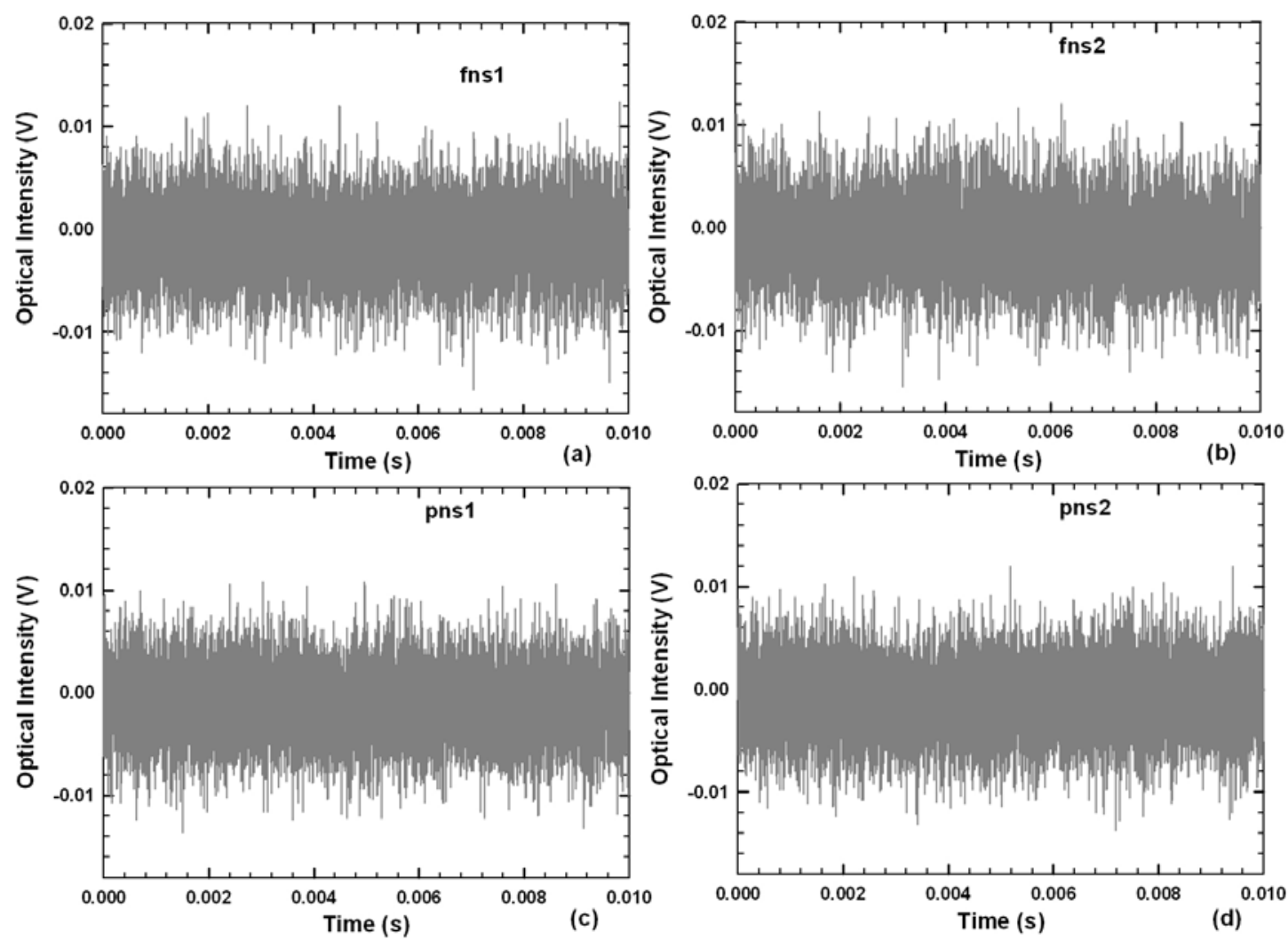

Figure 2-4: (a) —fns1, noise removed from optical signal (driving was 700Hz); (b) - fns2, noise removed from optical signal (driving was $400 \mathrm{~Hz}$ ); (c)—pns1, pure noise signal from optical through wafer setup; (d)-pns2, another peace of pure noise signal from optical through wafer setup

The histogram (Figure 2-5) of the four pieces of data shows that they all follow Gaussian distribution. The means and standard deviations for the four pieces of data have been calculated, and are shown in Figure 2-5. We can see that these values for different signals are very close. The similarity of these signals inferrs that the source of these signals might be same.

More time domain statistical analyses (autocorrelation -- Figure 2-6, cross correlation -- Figure 2-7) have been performed and they all showed good alignment with the assumption we just made, which is the four pieces of data are similar and could be from the same source. 

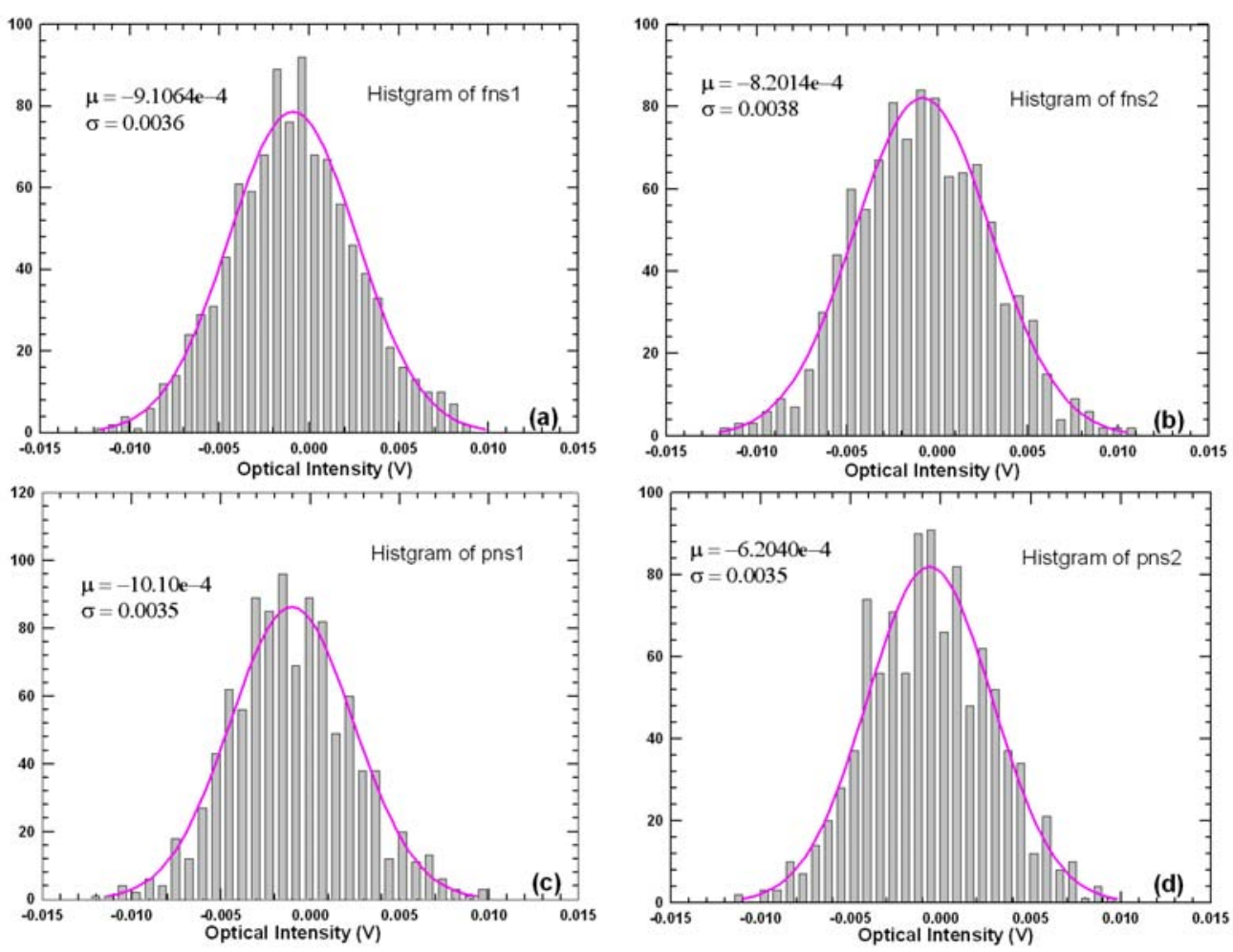

Figure 2-5: Histograms of (a) fns1; (b) fns2; (c) pns1; and (d) pns2
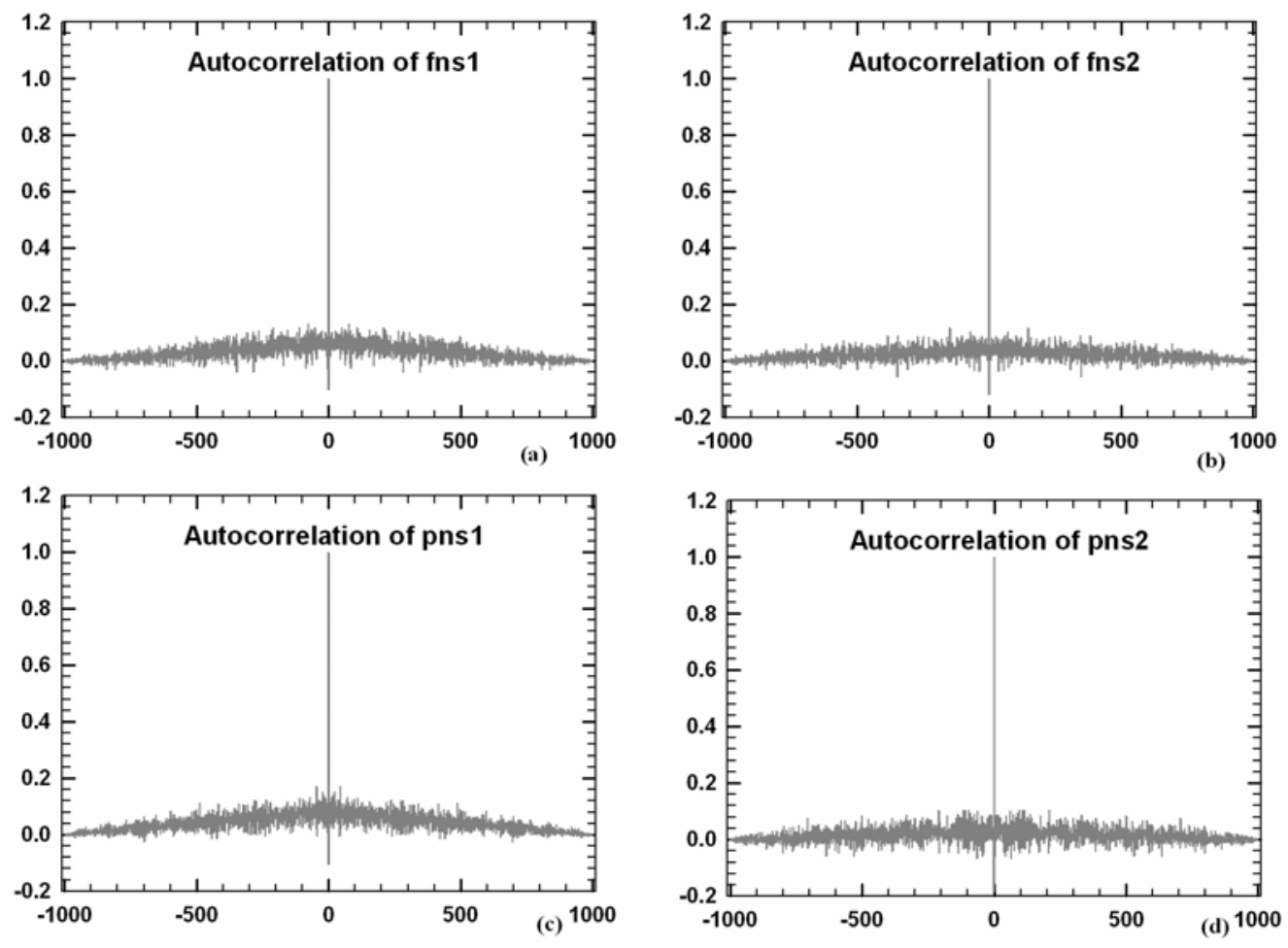

Figure 2-6: Autocorrelation of (a)—fns1; (b)—fns2; (c)—pns1; and (d)—pns2 

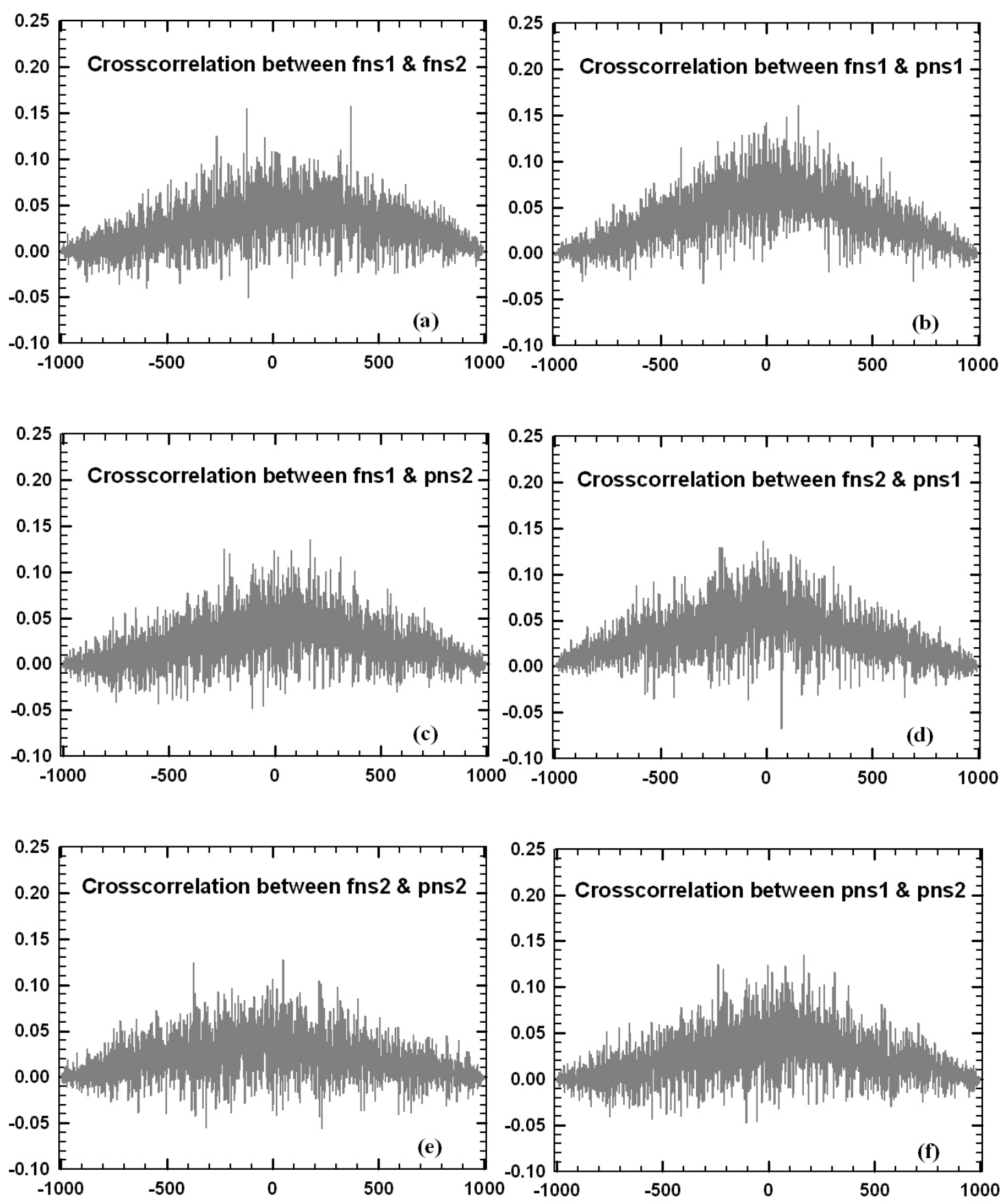

Figure 2-7: Cross correlation results between noise signals

The similarity of those signals also exists in the frequency domain. The FFT spectrum of all the four pieces of noise has been plotted (Figure 2-8). The plots show that no dominant frequency exists in any of them, and they look just like the copies of one another. The cross correlation between these four FFT signals has been performed, the 
results (Figure 2-9) indicate that they are highly correlated, in other words, the four pieces of data highly coincide to each other in frequency domain.
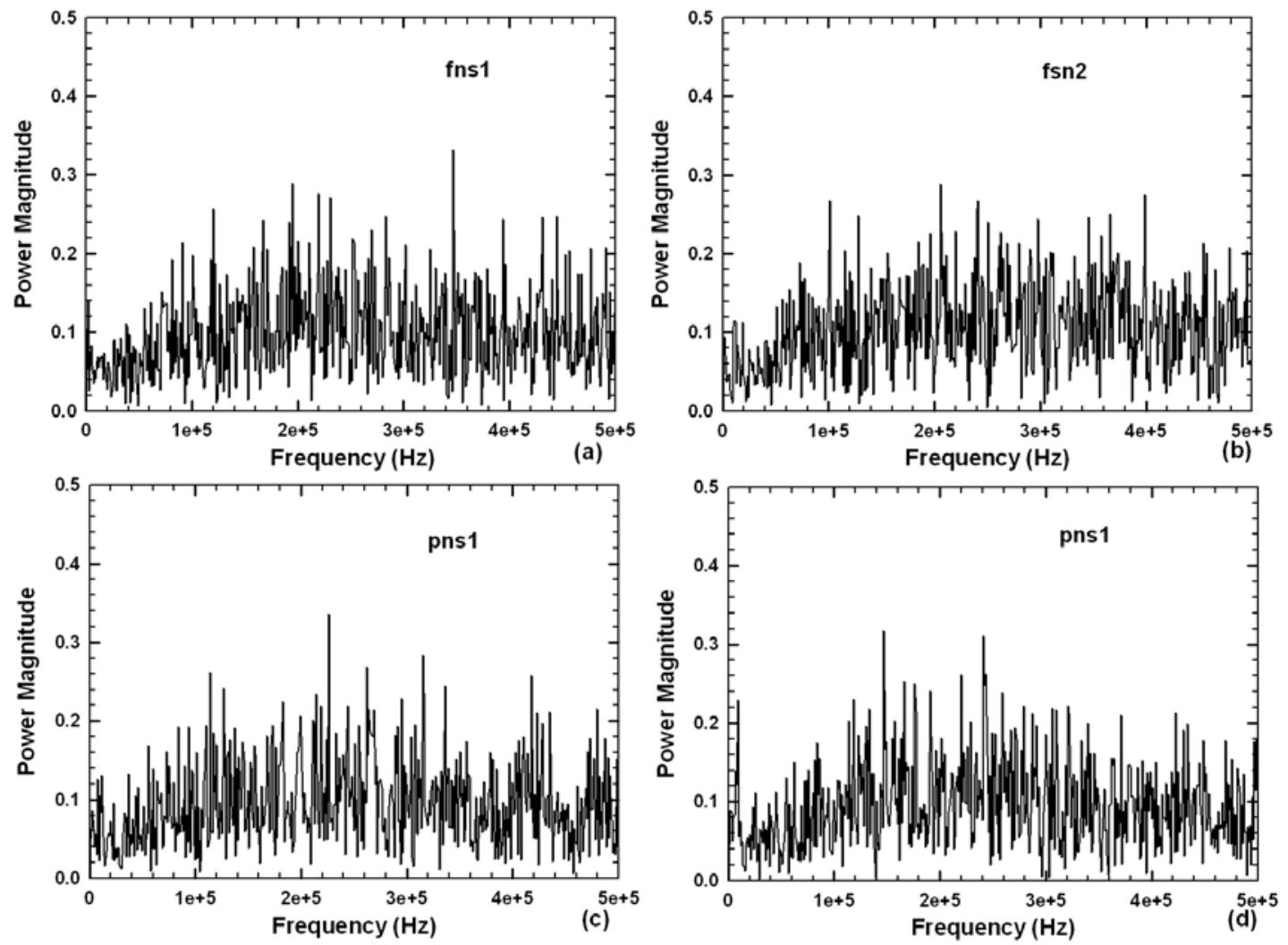

Figure 2-8: FFT Spectrum of (a) fns1; (b)—fns2; (c)—pns1; (d)—pns2 

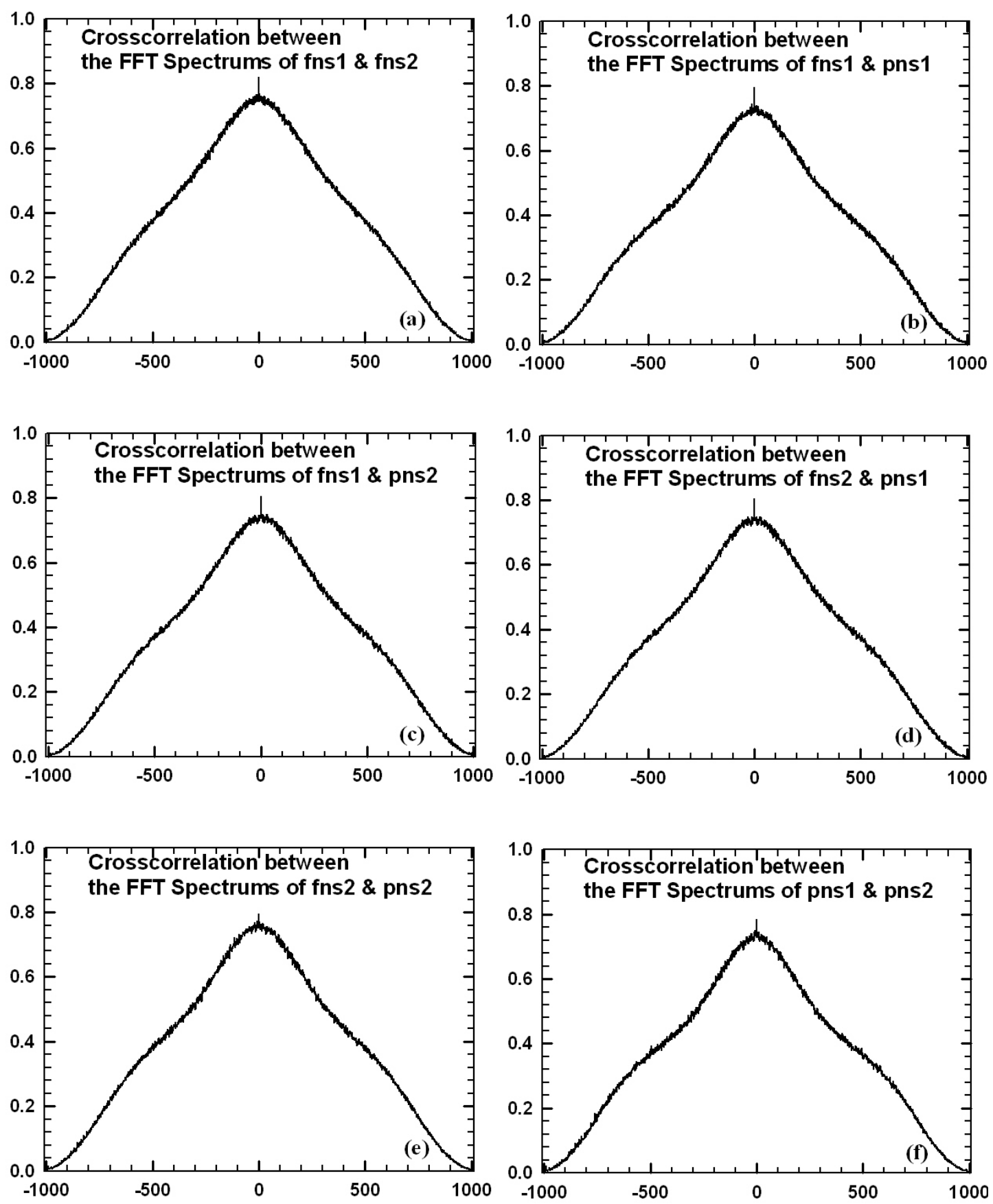

Figure 2-9: Cross correlation results between FFT spectrums of noises

Based on these analyses, it can be concluded that the noise in the optical signal is mainly stems from the optical through-wafer setup. 


\subsubsection{Conclusion}

From the result of analysis, fns1 and fns2 have same characteristics as pns1 and pns2 in both time and frequency domains, two conclusions can be deduced: 1) the shuttle movement of single frequency driven LCR can be represented by harmonic' components of the through wafer optical signal; 2) the noise that recorded from the optical throughwafer setup can be treated as the noise that exists in the optical signal.

\subsection{Noise Filtering}

From previous analysis, the clear optical signal for single frequency driven LCR can be easily obtained by the $\mathrm{K}$ times frequencies rule. However, when the driving signal contains more than one frequency, the clear optical observation for the shuttle displacement cannot be achieved in the same way, more common noise reduction methods have to be considered.

\subsubsection{Brief Introduction on Noise Reduction}

More than a hundred noise-reducing methods have been developed by researchers, and they can be categorized into three groups: statistical approach, spatial smoothing approach, and transform domain (TD) noise reduction approach.

Instead of performing noise reduction on the signal directly, the statistical method deals with function estimation from noisy signals [39]. This method requires an accurate noise model, and also because the computing complexity, computation time will exponentially increase when the order of noise model increases. Therefore this method is not suitable for the noise appearing in the signal that is a Gaussian distributed random signal, especially when the mean of the noise is not zero.

The spatial smoothing method uses a spatial filter as a smoothing mask and is applied primarily to local noise data [40]. It is also called a low-level signal processing algorithm. Spatial filters have been used for a long time to remove noise from images and signals as the traditional way [41]. These filters reduce noise by means of smoothing data, however, they also cause a portion of signal loss from the signal itself (blur the data) [42]. The Savitzky-Golay smoothing filter, a famous member of the Spatial Smoothing 
filter family, has been implemented to evaluate the noise reduction effect for the noise corrupted optical signal in this study. The evaluation result will be discussed later.

The idea for transform domain (TD) noise reduction is based on the consideration, that, although most of the information can be revealed from a signal's time domain representation directly, there still exist a chance that some of the information in that signal can only be found from other domains. For a signal, if the noise appears in its time domain, the noise must also show up in other domains, such as frequency domain, for that signal. By applying mathematical transformations on the time domain signal, more information that is not apparent in its original time domain can be found.

Generally, the transform domain noise reduction method is implemented in three steps:

- Transformation of input data by an orthogonal transforms.

- Modification of transformed coefficients by a nonlinear algorithm.

- Inverse transformation of the modified coefficients.

Choosing a proper domain transformation method is the first task for Transform Domain noise reduction. The major transformation methods have been used under this category including:

- KLT (Karhunen-Loeve Transform),

- DCT (Discrete Cosine Transform),

- MLT (Modulated Lapped Transform),

- FT (Fourier Transform), and

- WT (Wavelet Transforms).

The Karhunen-Loeve Transform (KLT) method can be used to compress sets of correlated visual data $[43,44]$ because it provides the best approximation for a fixed rank stochastic signal and consequently achieves the goal of noise reduction. This method also has been successfully used for data compression in the communication industry. However, since KLT does not reduce noise directly, its noise suppressing capability is very limited comparing with other transformation methods $[45,46]$.

The Discrete Cosine Transform (DCT) method is mainly used for noise reduction on images in current International Standard formats, such as JPEG (Joint Photographic Experts Group) and MPEG (Motion Picture Experts Group). DCT helps to separate 
image into parts or spectral sub-bands with different levels based on the image's visual quality. DCT transforms a signal from the time domain to the frequency domain which is similar to the discrete Fourier Transform. The capability of image edge preserving noise reduction, which is implemented by filtering the image with a moving window in the DCT domain, has been proved $[47,48]$. The highest noise suppression capability can be achieved by applying a special moving window on each distinct image. However, this method does not have any advantage for 1-D signals.

Compared with DCT [ 49], Modulated Lapped Transform (MLT) has the advantage of non-blocking artifacts which make it suitable for applications like noise reduction on broadband signals, i.e. audio signals. MLT has been used for noise reduction on signals in International Standard AC-3 (within MPEG-2) format for high-quality digital audio compression. Because of the poor time resolution of the basis functions, MLT may introduce ringing artifacts at low bit rates. This is a disadvantage of the MLT method, which can affect its performance [50]. Like DCT, this method has no advantage in reducing noise for 1-D signal.

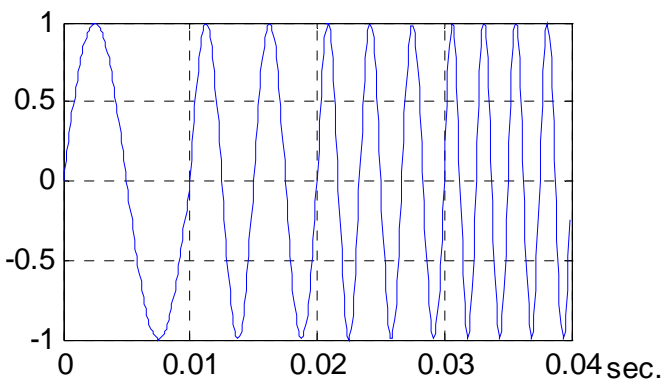

(a)

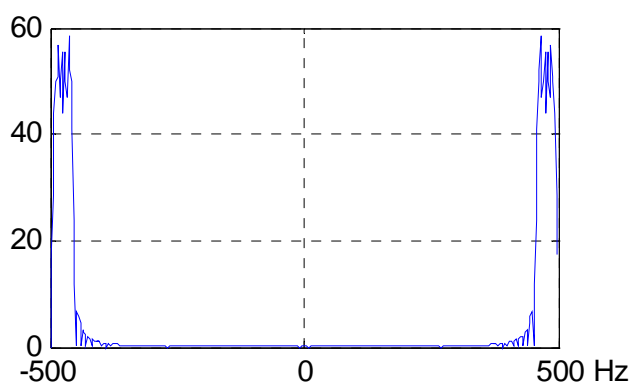

(c)

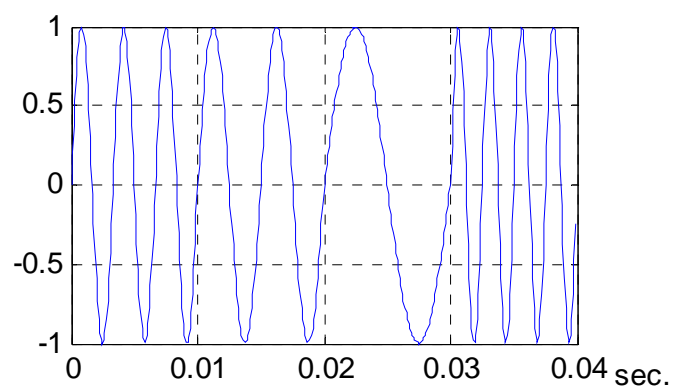

(b)

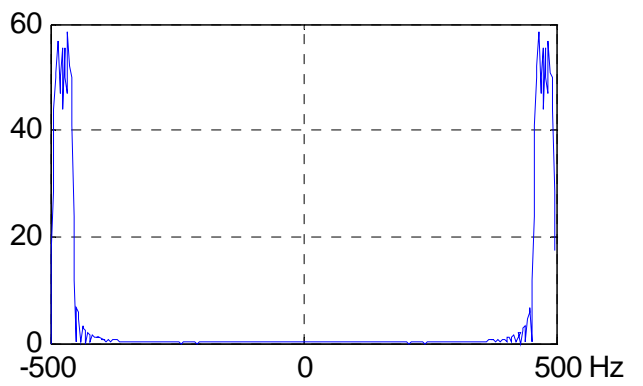

(d)

Figure 2-10 (a)Signal A, (b)Signal B, (c)Frequency Spectrum of Signal A, (d)Frequency Spectrum of Signal B 
The frequency spectrum of a signal, which can be obtained by applying the Fourier transform (FT), shows the frequency components of that signal. In traditional Fourier transform based signal processing, the spectrum of the signal is assumed to have little overlap with spectrum of the noise. Therefore linear time-invariant filtering is employed. This linear filtering method cannot separate noise from signal if the Fourier spectra overlap. Besides, FT is not a suitable technique for non-stationary signal noise reduction [51] because the frequency spectrum can only show what frequencies exist but can not tell when they appear, i.e., no time domain information. For example, signal A and signal B in Figure 2-10 are different in time domain, their frequency spectra are exactly the same.

Fortunately, the noise that exists in our optical signal is stationary, and based on previous analysis, the spectrum overlap between the signal and the noise is very small. Therefore, the Fourier transform based noise reduction approach can be a good candidate in this study.

By observing the FFT spectrum of the noise signal (Figure 2-8 (a) (b)), we can easily find that more than $95 \%$ of the noise components sit in the frequencies beyond $25000 \mathrm{~Hz}$. Since the frequencies of the signal that we are dealing with are far less than $25000 \mathrm{~Hz}$, low pass filter might be a good choice.

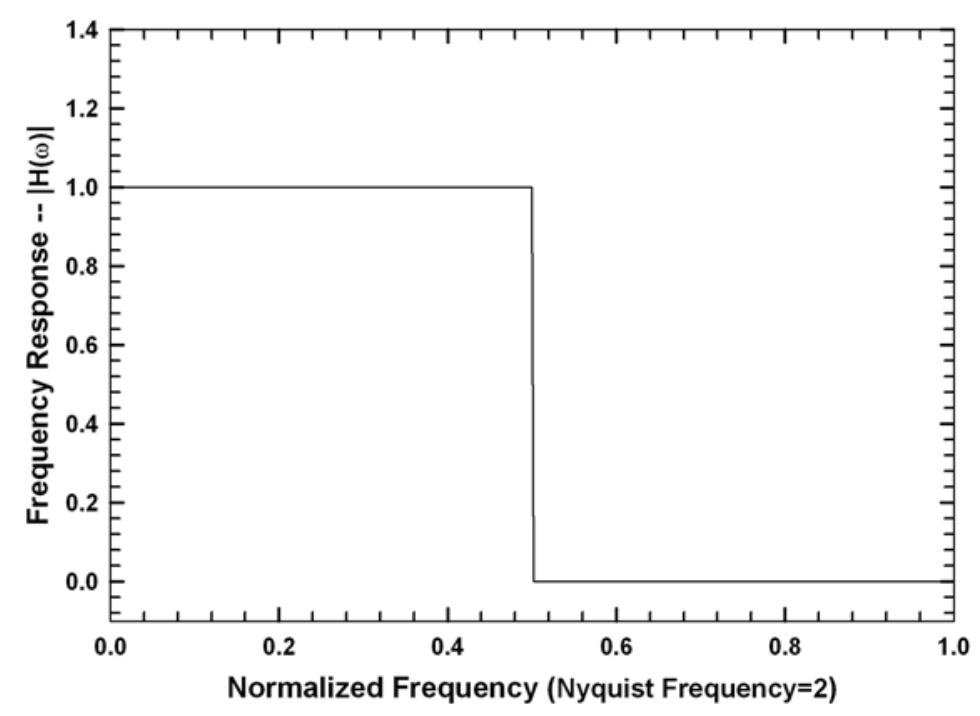

Figure 2-11: Magnitude response of ideal lowpass filter 
For an ideal low pass digital filter (Figure 2-11), if the cutoff frequency is $\omega_{c}(\mathrm{rad} / \mathrm{s})$, it can be described like

$$
\text { Equation 2-1: } H(\omega)=\left\{\begin{array}{cc}
1 & |\omega| \leq \omega_{c} \\
0 & \omega_{c}<|\omega| \leq \pi
\end{array},\right.
$$

\section{AND THE IMPULSE RESPONSE OF WHICH IS}

Equation 2-2: $\quad h(n)=\frac{1}{2 \pi} \int_{-\pi}^{\pi} H(\omega) e^{j \omega n} d \omega=\frac{1}{2 \pi} \int_{-\omega_{c}}^{\omega_{c}} e^{j \omega n} d \omega=\frac{\omega_{c}}{\pi} \sin c\left(\frac{\omega_{c}}{\pi} n\right)$.

However, since the impulse response is infinite and is noncausal ${ }^{1}$, this ideal filter cannot be implemented physically. To avoid the infinite and noncausal sequence, the frequency response $H(\omega)$ of the filter cannot have an infinitely sharp frequency cutoff at $\omega_{c}$. Although the ideal filter cannot be realized in the real world, an approximation (Figure 2-12) of the ideal filter can be realized by relaxing the sharp cutoff condition and allowing ripples exist in the passband and stopband. This filter can be further optimized by applying a window function on it. In this study, this kind of low pass filter has also been implemented to evaluate the noise reduction effect and the result will be discussed later.

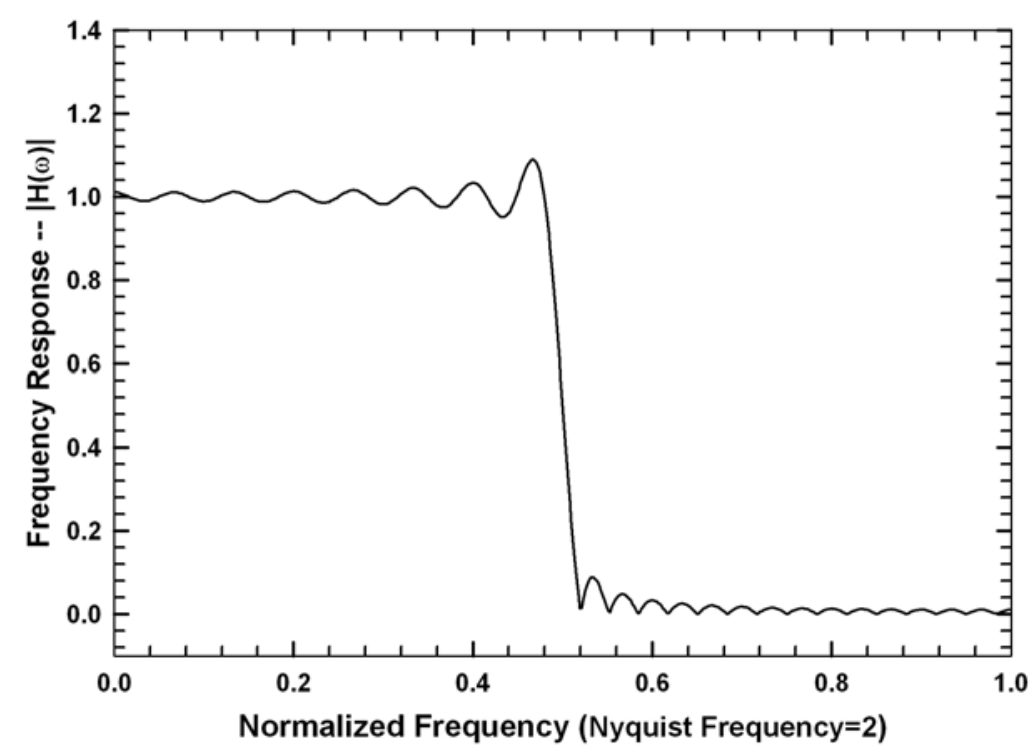

Figure 2-12: Magnitude response of physically realizable lowpass filter

\footnotetext{
${ }^{1}$ For a sequence $x(n)$, if $x(n)=0$ when $n<0$. we call it casual sequence; if for both $n<0$ and $n>0, x(n)$ are none zero, this sequence will be called noncasual [pp87, Digital Signal Processing].
} 


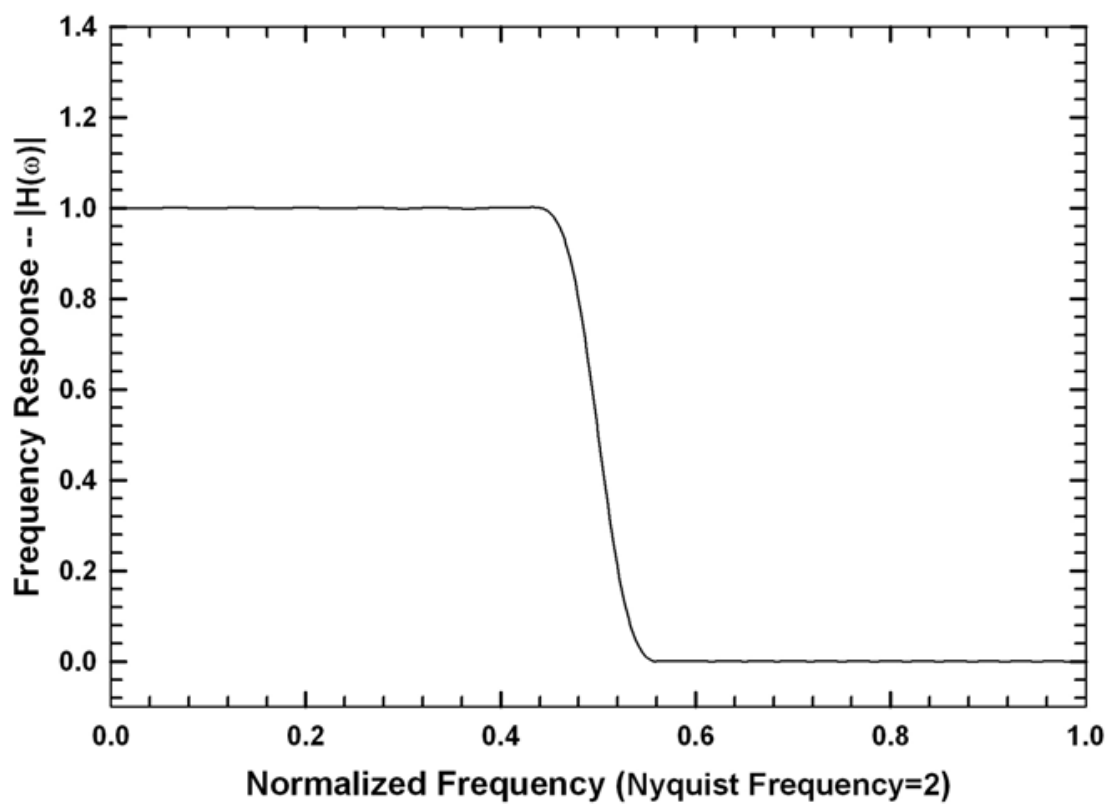

Figure 2-13: Magnitude response of physically realizable filters with Hamming window

The wavelet Transform (WT) method can compensate for the problem that is caused by the spectrum overlap in the FT method. WT can be regarded as a decomposition of a signal in the time-scale plane. It is an efficient technique for signal processing on non-stationary signals (time-varying spectra) because it can provide the time-frequency representation. The idea for Wavelet Denoising is based on the assumption: after the wavelet transform, the data is decomposed into different bases, where 'larger' coefficients correspond to the signal, and 'smaller' coefficients mostly represent the noise. This method allows clipping, thresholding, and shrinking of the amplitudes of the coefficients, which are used for separating signals or removing noise. The procedure of wavelet denoising is shown in Figure 2-14.

Wavelet shrinkage denoising (such as thresholding) is not smoothing. Instead, it removes the high frequency signals and keeps low, and "attempts to remove whatever noise is present and retain whatever signal is present regardless of the frequency content of the signal”[52].

Thresholding in the wavelet domain is due to selectively removes coefficients of wavelet transform in the measured signal. Thresholding generally gives a lowpass and "smoother" version of the original noisy signal. The objective of thresholding is to 
suppress the additive noise from the signal. There are two popular types of thresholding, one is based on hard thresholding and the other is based on soft thresholding. The signal is decomposed into L-levels of wavelet transform at first, then, the threshold $\delta_{i}$ for each level (in soft thresholding) or a threshold $\delta$ for all levels (in hard thresholding) are selected. The threshold $\delta$ is used to compare with all the detailed coefficients. It is known that soft thresholding provides smoother results in comparison with the hard thresholding techniques [53]. Noise reduction with wavelet thresholding has also been implemented in this study, and the result will be discussed later.

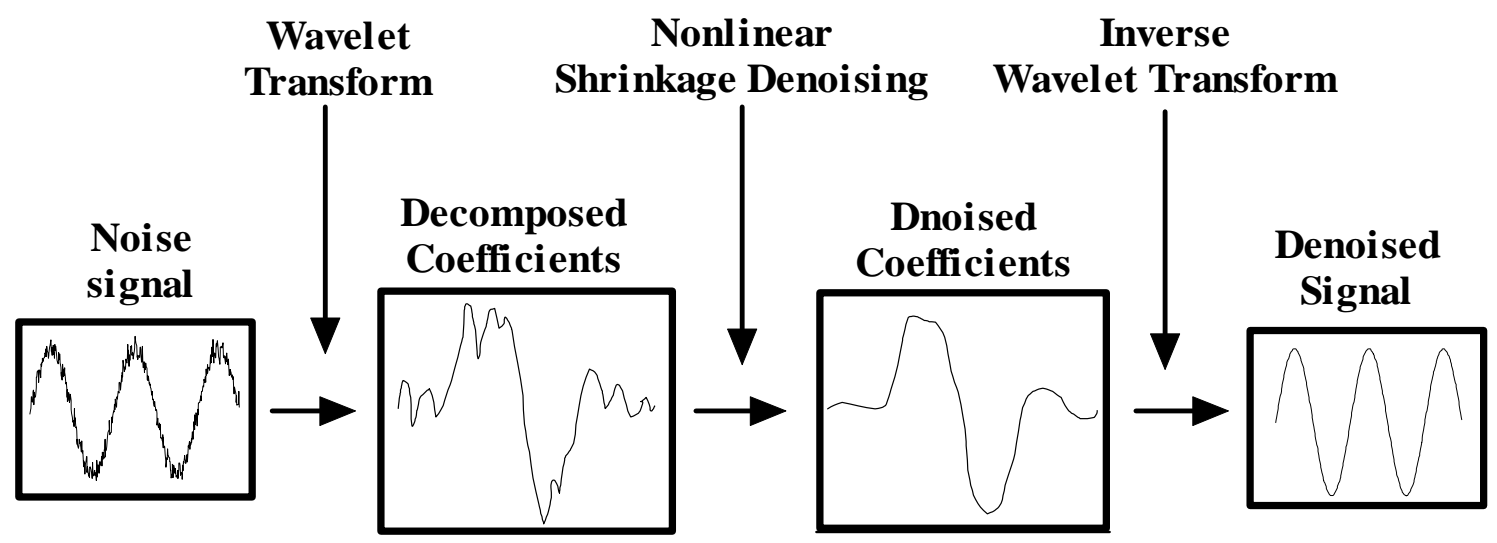

Figure 2-14: Procedure of Wavelet Denoising

\subsubsection{Noise Reduction of Optical Signal}

A high performance noise reduction method should filter out the noise components as much as possible and minimize information loss in the signal. Furthermore, for the future real time application of the MEMS LCR control, the noise reduction method should have the ability of being excuted within reasonable time. Three candidates have been carefully selected from the three categories mentioned in Section 2.1, which are frequency domain low pass filter, Savitzky-Golay smooth filter, and wavelet thresholding.

A simulation has been designed to compare the performance of the three candidates. In this simulation, all three methods have been performed on a noisecorrupted signal (a clear signal plus the noise signal recorded from experiment). Then the 
signal noise ratios of the noise reduced signals and the cross correlations between the clear signal and the filtered out noises have been compared.

A noise corrupted signal CS (Figure 2-16) has been generated by overlapping the noise signal that we discussed in section 2.1 to a clear signal S (Figure 2-15). For this corrupted signal, the noise reduction results of the three candidates are shown in Figure 2-17. The errors between the original clear signal and noise-reduced signals are also shown in Figure 2-18.

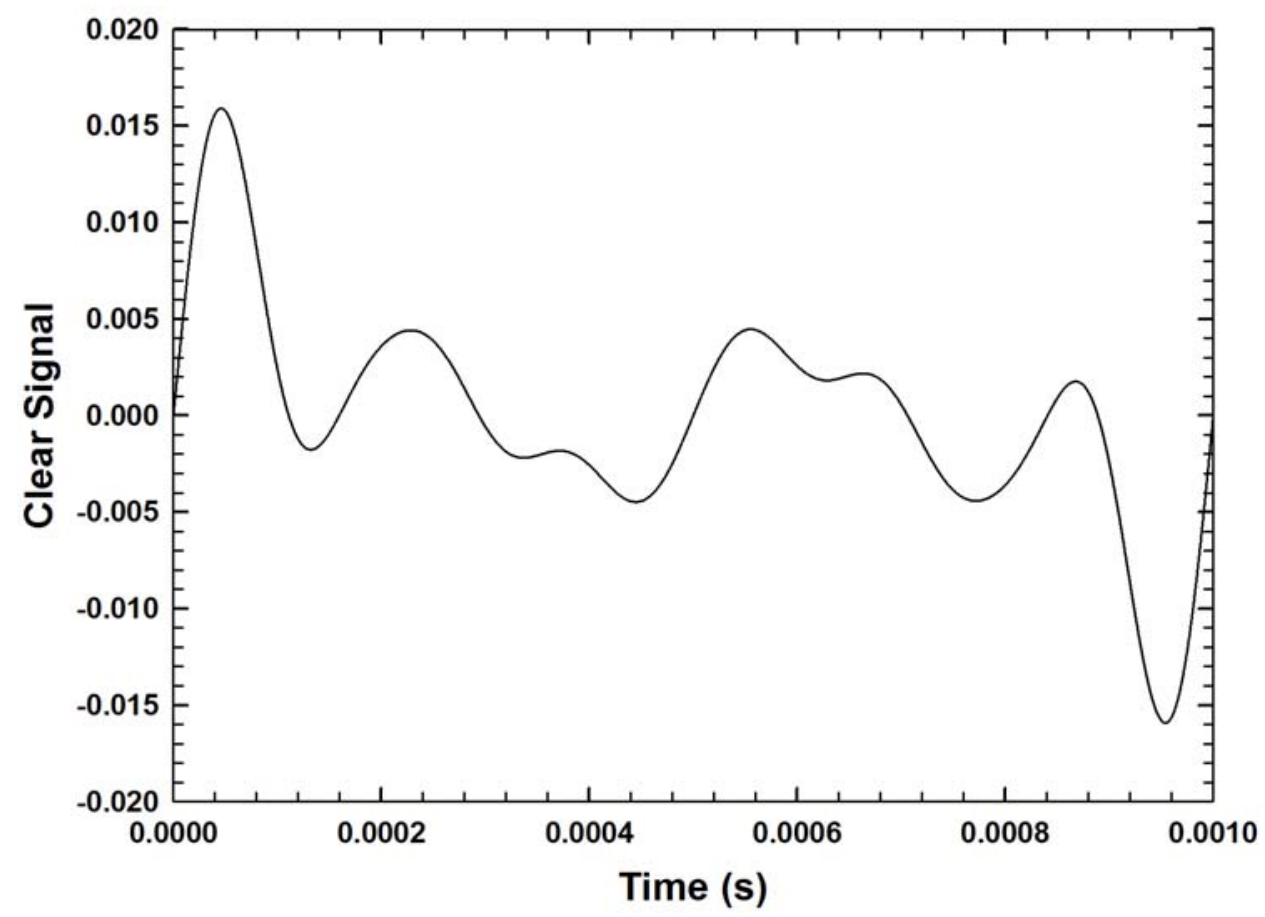

Figure 2-15: Clear Signal S that used to evaluate performance of the three noise reduction methods 


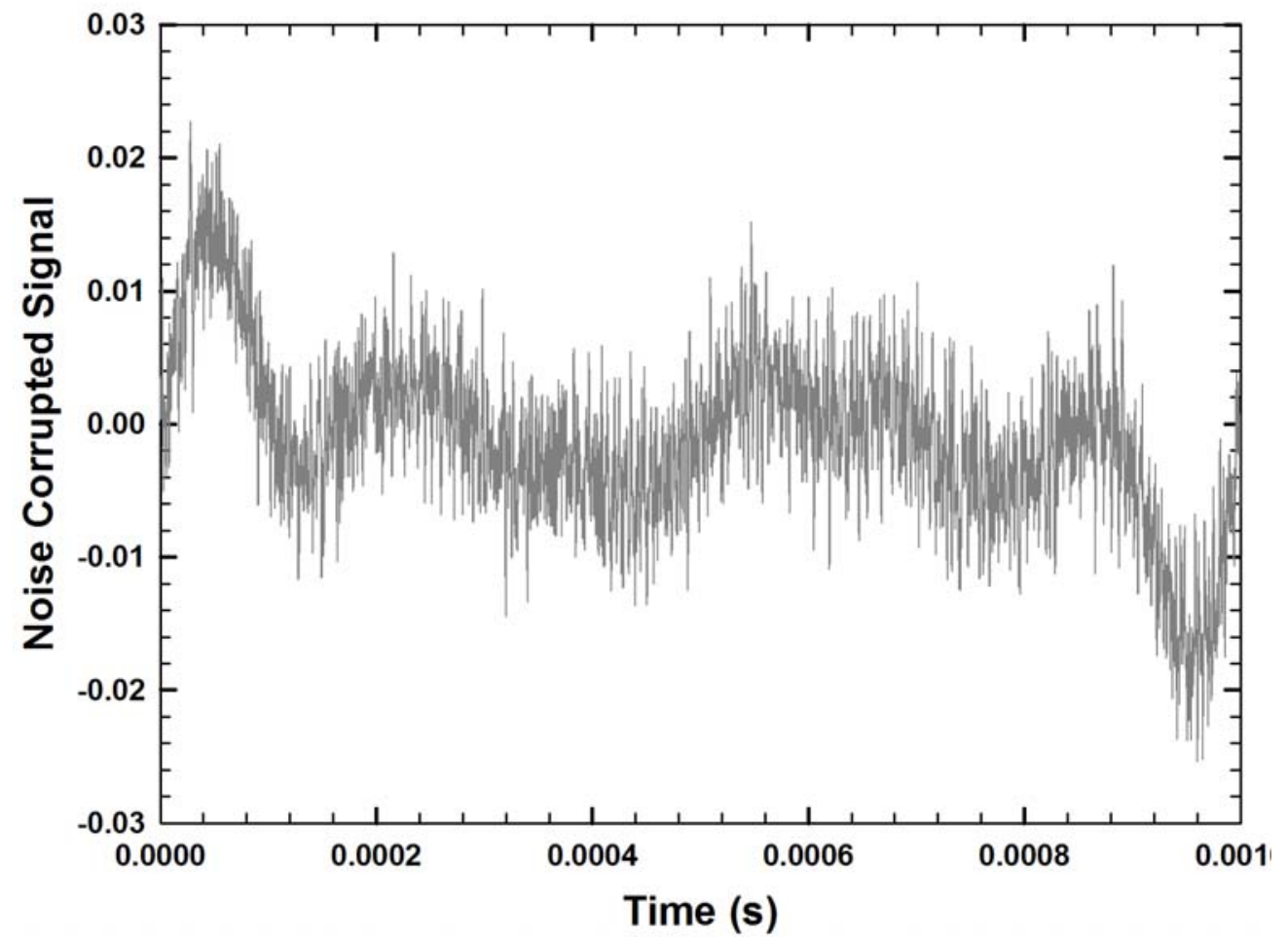

Figure 2-16: Noise Corrupt Signal $C_{S}$

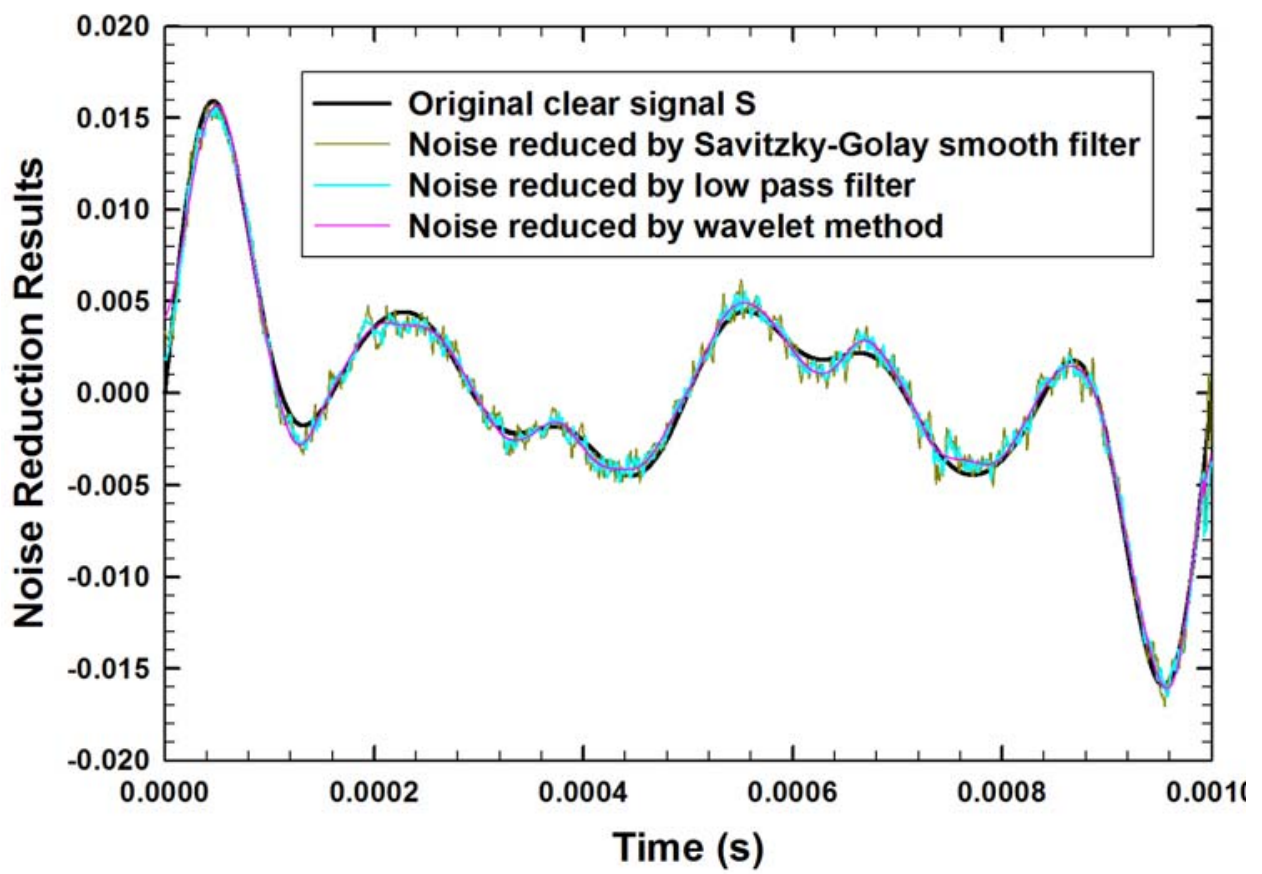

Figure 2-17: Noise Reduction Results by Three Noise Reduction Candidates 


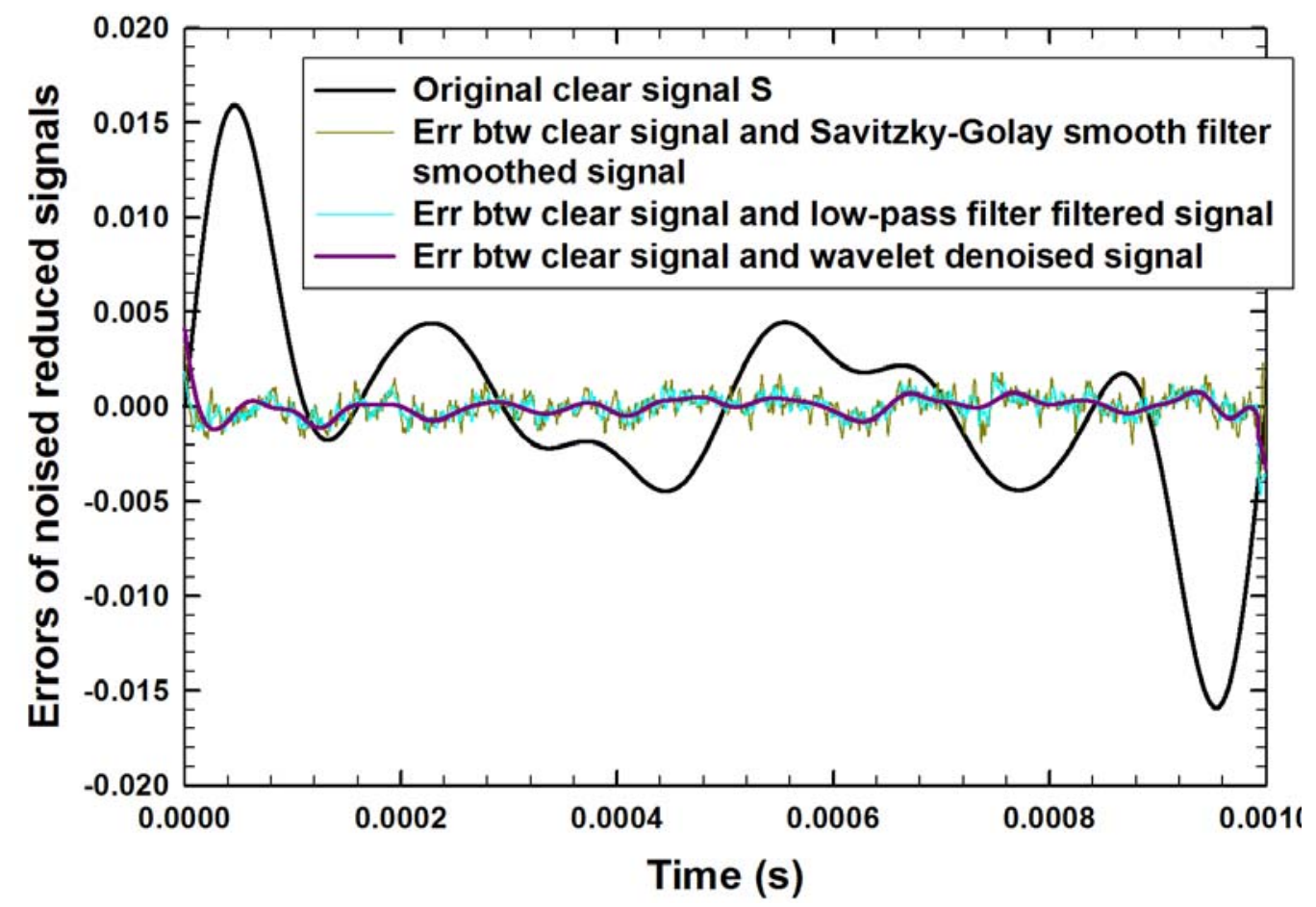

Figure 2-18: Errors Between Denoised Signals and Original Signals

Although it seems that wavelet thresholding method has slightly better performance than others (from Figure 2-17), more work has to be done to prove it. 


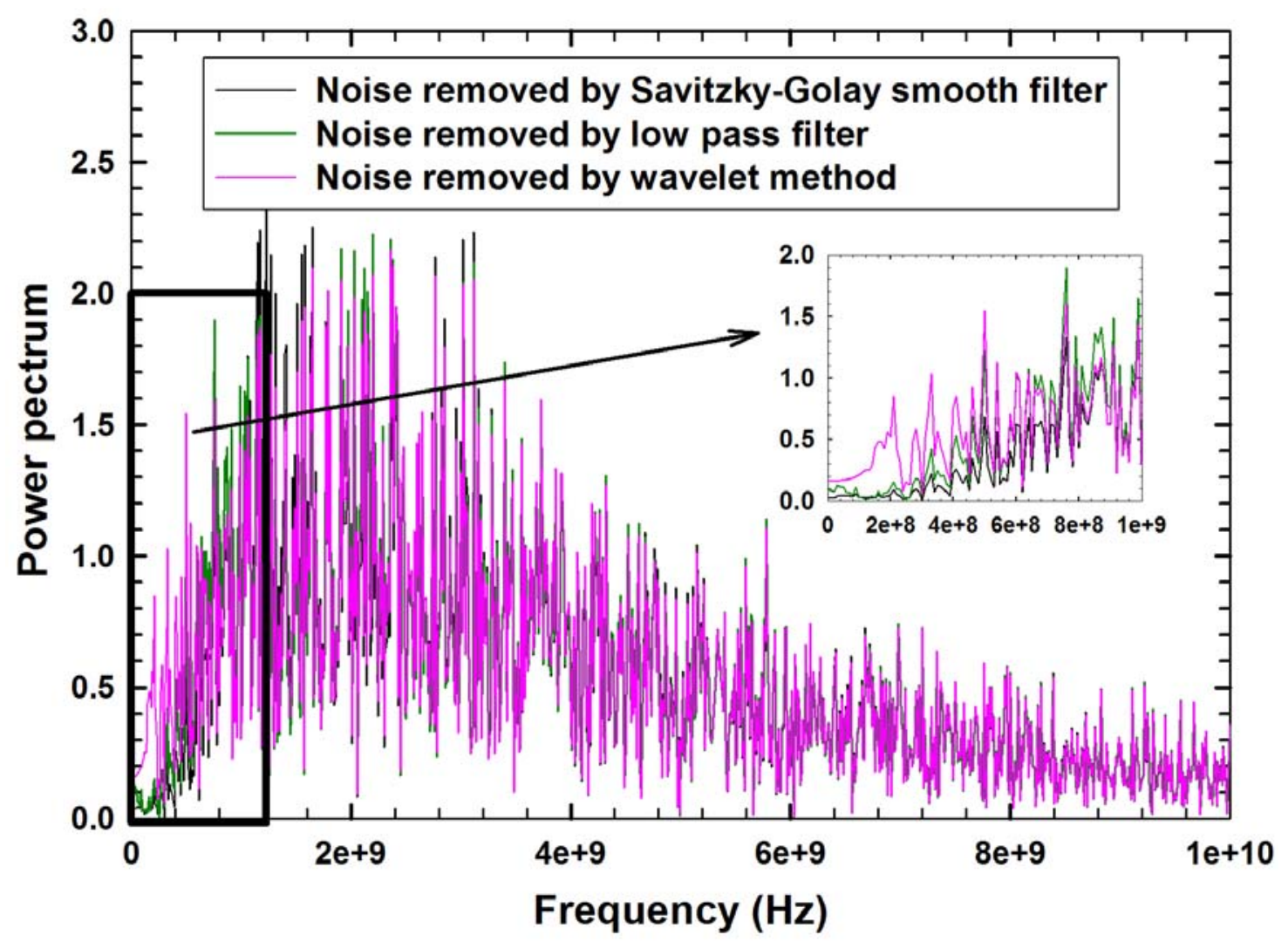

Figure 2-19: Power spectrum of removed out noises with the three methods

First, the noise signals that were removed by the three methods have been analyzed to compare performance on information loss. From the power spectrum, it is hard to tell if there is any useful information that has been removed for all three methods. Even if there were, the power spectra of the three are matched so well in the plot, we still cannot get a confident conclusion as to which one has better performance. However, by examining the lower frequency part of the power spectrum, a difference between wavelet method and the other two methods has been captured; the wavelet method removes more information than the other two while the Savitzky-Golay smooth filter removes less information than others. Cross correlation between clear signal S and the noises that were removed by the three methods has also been performed (Figure 2-20). It also shows the high similarity between these noise signals, which means although the wavelet method removes more information at lower frequency than the others, it is not based on the 
sacrifice of more useful information loss from the objective signal. Therefore, the wavelet method is more efficient than the other two methods in the lower frequency range.

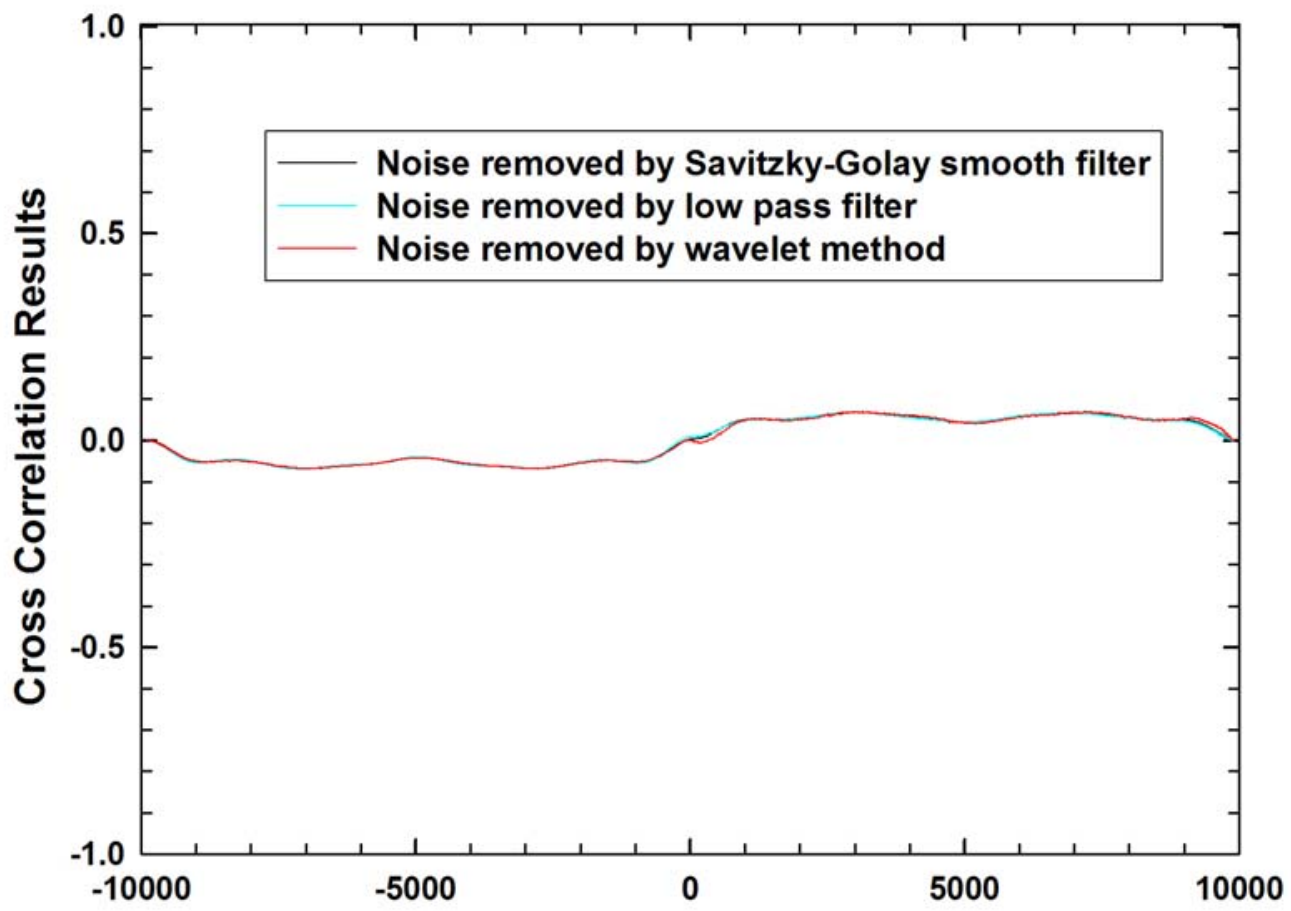

Figure 2-20: Cross correlation results between removed out noise signals and original clear signal

SNR of the noise reduced signals, and the square errors between original clear signal and the noise-reduced signal were calculated. The results are shown in Table 2-1.

Table 2-1: SNR and square errors between clear signal and noise reduced signals

\begin{tabular}{|c|c|c|}
\hline Noise Removing Method & SNR (dB) & Square errs. \\
\hline No method applied & 3.748 & $1.3174 \mathrm{e}-1$ \\
\hline Low-pass filter & 20.744 & $2.6457 \mathrm{e}-3$ \\
\hline Savitzky-Golay smooth filter & 18.535 & $4.4008 \mathrm{e}-3$ \\
\hline Wavelet Thresholding & 21.333 & $2.3105 \mathrm{e}-3$ \\
\hline
\end{tabular}

\subsubsection{Discussion}

Both SNR and square errors show that the Wavelet Thresholding method has the best noise reduction performance among the three candidates, i.e., it has the highest SNR and smallest square error. However, the wavelet thresholding method requires extension calculations, which cannot meet the computation cycle time requirement for real-time 
applications. Therefore, the wavelet thresholding method can only be used for those offline applications such as modeling. Since both lowpass filter and wavelet thresholding methods have much better performance than the Savitzky-Golay filter method, and the algorithm for the low-pass filter is simple enough to be implemented in reasonable time, for the real-time on-line applications involved in this study, the faster low-pass filter will be utilized. 


\section{Chapter 3 System Modeling}

A mathematical description for the lateral comb resonator is necessary for any control routine to be applied to it. Under no load condition, the mathematical model for the electrostatic driven LCR (Figure 3-1) can be described as [54]:

Equation 3-1

$$
F_{e}=m \ddot{x}+\beta \ddot{x}+2 k_{s} x,
$$

where:

$m$ : effective mass of the moving stage,

$x$ : displacement of the shuttle,

$k_{s}$ : spring constant,

$\beta$ : damping coefficient, and

$F_{e}$ : electrostatic force (generated by the comb capacitor).

Most of the parameters that are involved in this mathematical model can be estimated through physical analysis, and can be experimentally confirmed by performing system identification based on measured input/output data. In this study, in order to get the above mentioned parameters, the theoretical analysis based on the device's physical properties was performed first. Then several experimental methods that can be used to perform the parameter identification were discussed and performed.

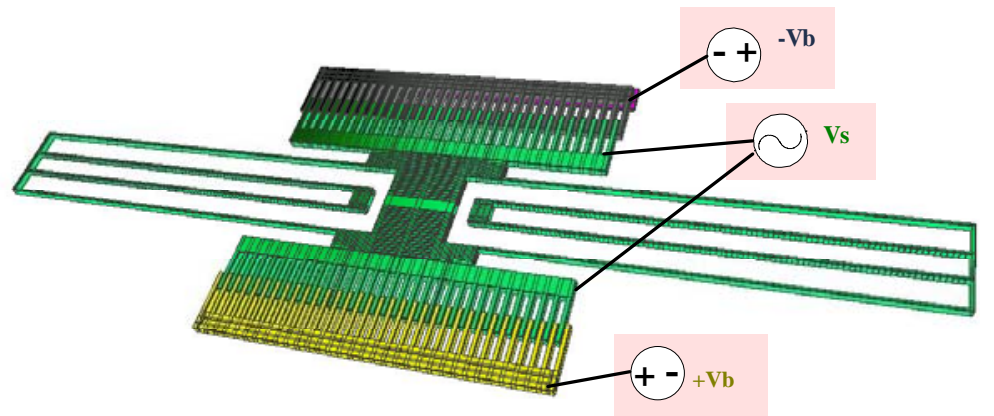

Figure 3-1 Schematic drawing of the MEMS Comb Resonator designed by WVU 
If the absolute value of the DC voltages that are applied to the stators (side combs) are equal (with opposite signs), then the electrostatic force that is generated by the overlapping finger is linear and can be obtained by [55,56]

$$
\text { Equation 3-2 } \quad F_{e}=-2.24 n \varepsilon \frac{t_{v}}{g}\left(V_{b} V_{s}\right) \text {, }
$$

where:

$$
\begin{aligned}
& n \text { : number of teeth on the stage comb, } \\
& \varepsilon \text { : permittivity of air, and } \varepsilon=8.854 \times 10^{-12}, \\
& t_{v} \text { : vertical thickness of teeth, } \\
& g \text { : comb finger-to-finger gap, } \\
& V_{b} \text { : DC voltage applied to the stator combs, and } \\
& V_{s:} \text { AC voltage applied to the translation stage comb. }
\end{aligned}
$$

For the symmetric structure LCR involved in this research, when the load is zero $\left(F_{d}=0\right.$ ), the system transfer function can be obtained by taking the Laplace Transform of Equation 3-1, as shown in the following equations:

$$
\text { Equation 3-3 } H(s)=\frac{x(s)}{F_{e}(s)}=\frac{1 / m}{s^{2}+\frac{\beta}{m} s+\frac{2 k_{s}}{m}},
$$

and

$$
\text { Equation 3-4 } \quad H(j \omega)=\frac{1 / m}{\left(\frac{2 k_{s}}{m}-\omega^{2}\right)+j \frac{\beta}{m} \omega},
$$

Furthermore, the resonance frequency of the system can be determined as:

Equation 3-5

$$
\omega_{n}=\sqrt{\frac{2 k_{s}}{m}}, \quad f_{n}=\omega_{n} / 2 \pi .
$$

\subsection{Theoretical Parameter Analysis}

Theoretically, the effective mass that contributes to the moving part can be calculated as [57] 


$$
\text { Equation 3-6 } m=m_{s}+\frac{1}{4} m_{t}+\frac{12}{35} m_{b} \text {, }
$$

where:

$m_{s:}$ stage mass (given in Table $3-1$ ),

$m_{t:}$ truss mass, and

$m_{b \text { : }}$ beam mass.

Table 3-1: Structure Parameters for Different Structure Comb Resonators Designed at WVU

\begin{tabular}{|c|c|c|}
\hline Structure & Mass of stage $\left.\mathbf{( m}_{\mathbf{s}}\right)$ & Spring length \\
\hline Grating & $1.827 \mathrm{e}-10 \mathrm{~kg}$ & $350 \mu \mathrm{m} / 400 \mu \mathrm{m} / 450 \mu \mathrm{m} / 500 \mu \mathrm{m}$ \\
\hline Poly2 & $2.006 \mathrm{e}-10 \mathrm{~kg}$ & $350 \mu \mathrm{m} / 400 \mu \mathrm{m} / 450 \mu \mathrm{m} / 500 \mu \mathrm{m}$ \\
\hline Poly2 \& metal & $2.152 \mathrm{e}-10 \mathrm{~kg}$ & $350 \mu \mathrm{m} / 400 \mu \mathrm{m} / 450 \mu \mathrm{m} / 500 \mu \mathrm{m}$ \\
\hline
\end{tabular}

The detailed beam and truss structure of the comb resonator is shown in Figure 3-2.

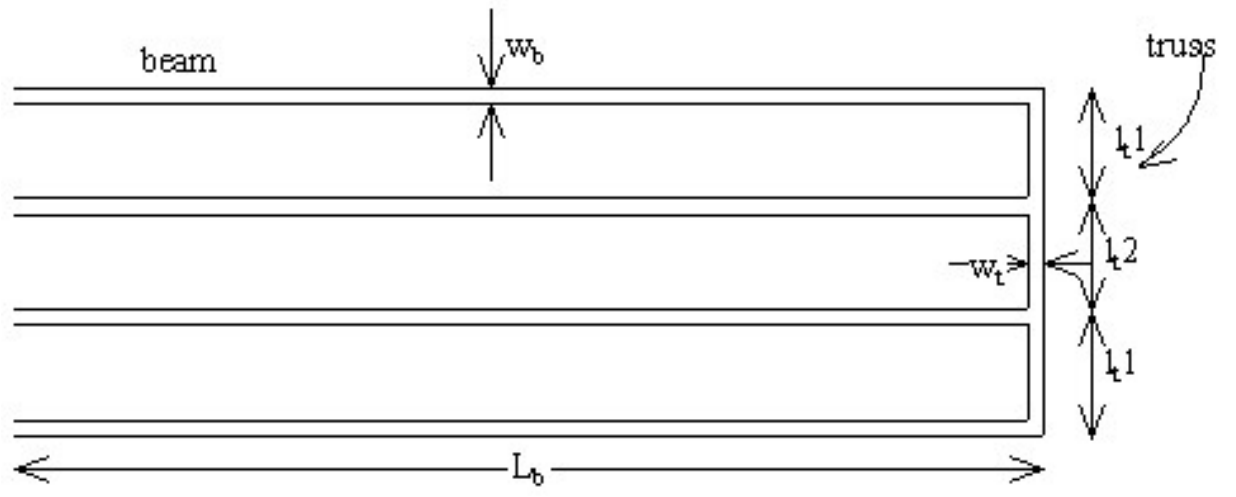

Figure 3-2 One Side Schematic Drawing of Beam and Truss of the Lateral Comb Resonator where:

$\mathrm{w}_{\mathrm{t}}=\mathrm{w}_{\mathrm{b}}=2 \mu \mathrm{m}$,

$\mathrm{L}_{\mathrm{b}}$ (beam length) $=350 \mu \mathrm{m} / 400 \mu \mathrm{m} / 450 \mu \mathrm{m} / 500 \mu \mathrm{m}$,

$l_{\mathrm{t} 1}=33 \mu \mathrm{m}$,

$\mathrm{l}_{\mathrm{t} 2}=28 \mu \mathrm{m}$,

Total truss length $\mathrm{L}_{\mathrm{t}}=2 \mathrm{l}_{\mathrm{t} 1}+\mathrm{l}_{\mathrm{t} 2}=94 \mu \mathrm{m}$, 
$\mathrm{t}$ (thickness of the structure) $=2 \mu \mathrm{m}$,

Density of polysilicon $=2.33 \times 10^{-15} \mathrm{~kg} / \mu^{3}$.

The spring constant $k_{s}$ can be calculated as [57],

$$
\text { Equation 3-7 } \quad k_{s}=\frac{2 E t_{v} w_{b}^{3}}{L_{b}^{3}}\left(\frac{L_{t}^{2}+14 \alpha L_{t} L_{b}+36 \alpha^{2} L_{b}^{2}}{4 L_{t}^{2}+41 \alpha L_{t} L_{b}+36 \alpha^{2} L_{b}^{2}}\right),
$$

where:

$$
\mathrm{E}=165 \mathrm{GPa}=165 \times 10^{9} \mathrm{~Pa} \text {, is the young's modulus for polysilicon, }
$$

and, $\quad \alpha=\left(\frac{w_{t}}{w_{b}}\right)^{3}=1$.

For the damping factor, it can be introduced [57] as:

$$
\text { Equation 3-8 } \quad \beta=\mu\left[\left(A_{s}+\frac{A_{t}}{2}+\frac{A_{b}}{2}\right) \cdot\left(\frac{1}{d}+\frac{1}{\delta}\right)+\frac{A_{c}}{g}\right] \text {, }
$$

where:

$\mu$ : viscosity of air,

$A_{s}$ : surface area of stage,

$A_{t}$ : surface area of truss,

$A_{b}$ : surface area of flexure beams,

$A_{c}$ : surface area of comb finger sidewalls,

$d$ : oxide thickness gap,

$\delta$ : penetration depth of the airflow above the stage, and

$g$ : comb’s finger-to-finger gap.

Since the dimensions of the LCR have been presented in all the three equations for calculating the system parameters, almost none of these parameters can be determined accurately for the fabricated device due to the fabrication process tolerance. Especially 
for the damping parameter $\beta$, a slight imprecision on the device's dimension will result in significant differences in the value of the damping parameter. Therefore, it is impractical to obtain the damping parameter merely from theoretical calculation. In order to confirm the system model, parameter estimation by means of experimental methods is necessary in this study.

A grating structure LCR with beam length of $450 \mu \mathrm{m}$ has been chosen as an example to show how the effective mass $m$, and spring constant $k_{s}$ are calculated theoretically. Since mass = volumexdensity, to calculate the mass of this structure, the volume of each beam and truss must be determined first

$$
\begin{aligned}
& \text { volume of beam, } V_{B}=\left(L_{b}-w_{t}\right) \times w_{b} \times t_{v}=(450-2) \times 2 \times 2=179 \mu \mathrm{m}^{3}, \\
& \text { volume of truss, } V_{s}=L_{t} \times w_{t} \times t_{v}=94 \times 2 \times 2=376 \mu \mathrm{m}^{3} .
\end{aligned}
$$

Each device has one stage, 8 beams, and 2 total trusses (Figure 3-2), which make the mass of the beams

$$
\begin{aligned}
& m_{b}=8 \times(2.33 e-15 \times 1792)=3.340288 e-11 \mathrm{~kg}, \text { and mass of the trusses } \\
& m_{s}=2 \times(2.33 e-15 \times 376)=1.75216 e-12 \mathrm{~kg} .
\end{aligned}
$$

By Equation 3-6, the effective mass for this structure will be

$$
\begin{aligned}
m & =m_{s}+\frac{1}{4} m_{t}+\frac{12}{35} m_{b} \\
& =1.827 e-10+1.75216 e-12 / 4+\frac{12}{35} \times 3.340288 e-11 \\
& =1.9459 \times 10^{-10} \mathrm{~kg},
\end{aligned}
$$

from Equation 3-7, the spring constant for this structure will be

$$
\begin{aligned}
& k_{s}=\frac{2 E t_{v} w_{b}^{3}}{L_{b}^{3}}\left(\frac{L_{t}^{2}+14 \alpha L_{t} L_{b}+36 \alpha^{2} L_{b}^{2}}{4 L_{t}^{2}+41 \alpha L_{t} L_{b}+36 \alpha^{2} L_{b}^{2}}\right) \\
& =\frac{165 e 9 \times 2 e-6 \times(2 e-6)^{3}}{450 e-6}\left(\frac{(94 e-6)^{2}+14(94 e-6)(450 e-6)+36(450 e-6)^{2}}{4(94 e-6)^{2}+41(94 e-6)(450 e-6)+36(450 e-6)^{2}}\right) \\
& =0.025234,
\end{aligned}
$$

and, the resonance frequency can be found by using Equation 3-5,

$$
f_{n}=\frac{\omega_{n}}{2 \pi}=\sqrt{\frac{2 k_{s}}{m}} / 2 \pi=\sqrt{\frac{2 \times 0.025234}{1.9459 e-10}} / 2 \pi=2563.1 \mathrm{~Hz} .
$$


The theoretical parameters of different structure resonators designed at WVU have been calculated, and are listed in Table 3-2.

Table 3-2: Theoretical parameters for MEMS resonators manufactured in WVU

\begin{tabular}{|c|c|c|c|c|c|c|c|c|c|c|}
\hline \multirow{2}{*}{\multicolumn{2}{|c|}{\begin{tabular}{c|c} 
Structure & $\begin{array}{c}\text { Beam } \\
\text { length } \\
(\mu \mathrm{m})\end{array}$ \\
\end{tabular}}} & \multicolumn{3}{|c|}{$\begin{array}{c}\text { Mass } \\
\left(10^{-10} \mathrm{~kg}\right)\end{array}$} & \multicolumn{3}{|c|}{$\begin{array}{c}\text { Spring constant } \\
K\end{array}$} & \multicolumn{3}{|c|}{$\begin{array}{c}\text { Resonant frequency } \\
(\mathrm{Hz})\end{array}$} \\
\hline & & Min* & Std. & Max* & Min* & Std. & Max* & Min* & Std. & Max* \\
\hline \multirow{4}{*}{ Grating } & 350 & 1.8944 & 1.9203 & 1.9506 & 0.0271 & 0.0519 & 0.0905 & 2482.0 & 3698.6 & 4500.2 \\
\hline & 400 & 1.9036 & 1.9331 & 1.9675 & 0.0185 & 0.0354 & 0.0618 & 2046.7 & 3045.6 & 3703.6 \\
\hline & 450 & 1.9331 & 1.9459 & 1.9844 & 0.0132 & 0.0252 & 0.0441 & 1724.8 & 2563.1 & 3115.3 \\
\hline & 500 & 1.9675 & 1.9587 & 2.0013 & 0.0097 & 0.0186 & 0.0325 & 1478.9 & 2194.8 & 2666.3 \\
\hline \multirow{4}{*}{ Poly2 } & 350 & 2.0734 & 2.0993 & 2.1296 & 0.0271 & 0.0519 & 0.0905 & 2482.0 & 3537.4 & 4500.2 \\
\hline & 400 & 2.0826 & 2.1121 & 2.1465 & 0.0185 & 0.0354 & 0.0618 & 2046.7 & 2913.6 & 3703.6 \\
\hline & 450 & 2.0918 & 2.1249 & 2.1634 & 0.0132 & 0.0252 & 0.0441 & 1724.8 & 2452.8 & 3115.3 \\
\hline & 500 & 2.1011 & 2.1377 & 2.1803 & 0.0097 & 0.0186 & 0.0325 & 1478.9 & 2100.9 & 2666.3 \\
\hline \multirow{4}{*}{$\begin{array}{c}\text { Poly2 \& } \\
\text { Metal }\end{array}$} & 350 & 2.2194 & 2.2453 & 2.2756 & 0.0271 & 0.0519 & 0.0905 & 2482.0 & 3420.4 & 4500.2 \\
\hline & 400 & 2.2286 & 2.2581 & 2.2925 & 0.0185 & 0.0354 & 0.0618 & 2046.7 & 2817.9 & 3703.6 \\
\hline & 450 & 2.2378 & 2.2709 & 2.3094 & 0.0132 & 0.0252 & 0.0441 & 1724.8 & 2372.6 & 3115.3 \\
\hline & 500 & 2.2471 & 2.2837 & 2.3263 & 0.0097 & 0.0186 & 0.0325 & 1478.9 & 2032.6 & 2666.3 \\
\hline
\end{tabular}

* The range of parameters calculated here are based on: the MUMPs technique used to fabricate the MEMS resonator has $\pm 0.3 \mu \mathrm{m}$ 's manufacturing tolerance, and the accuracy of the device size would affect the value of mass, spring constant, and the resonance frequency (Equation 3-6, Equation 3-7, and Equation 3-5).

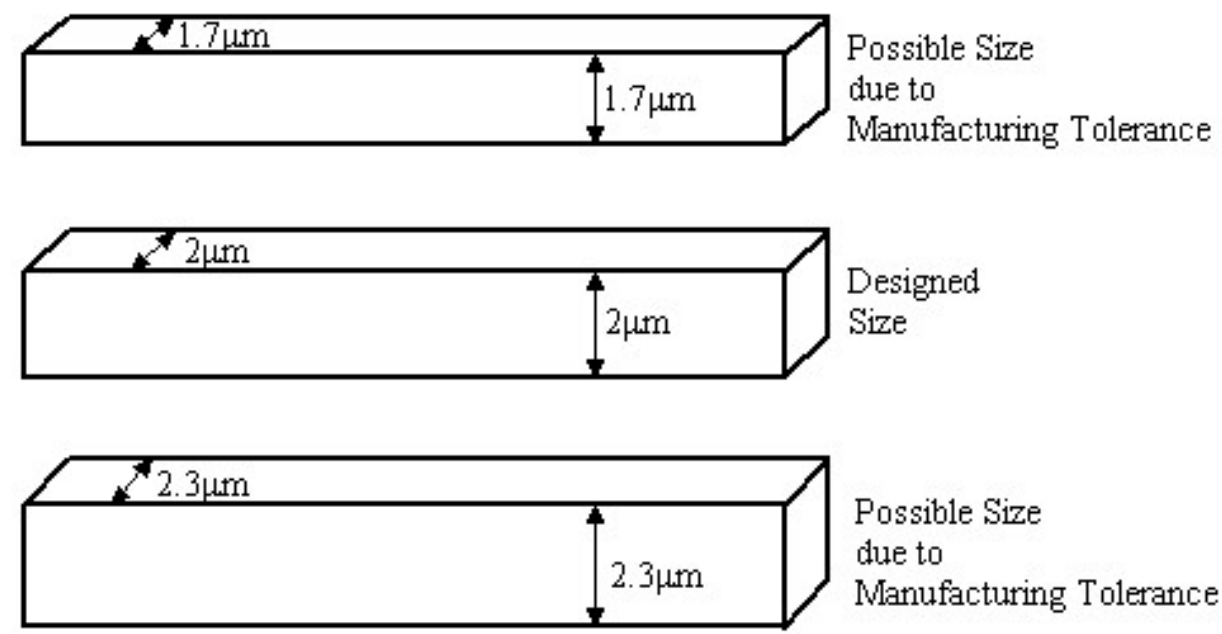

Figure 3-3 An example of the actual device sizes due to the manufacturing tolerance 


\subsection{Parameter Identification}

Parameter identification can be defined as: The experimental determination of values of parameters that govern the dynamic and/or non-linear behavior, assuming that the structure of process model is known (Eykhoff, 1974). There are many ways to perform parameter identification with experimental data, and parameter identification has been considered a powerful technique for building accurate models from noisy data. Generally, it can be categorized into two basic groups: time domain system identification, and frequency domain system identification. Parameter identification methods within both categories have been evaluated and implemented in this study.

\subsubsection{Time Domain System Identification}

Compared with the frequency domain method, usually, the time domain methods are more direct. Many time domain procedures have been surveyed [58,59,60,61,62,63], and two of them have been evaluated in this study, which are the Hilbert envelope method and the frequency sweeping method.

\subsubsection{Hilbert Envelope Method}

For a typical $2^{\text {nd }}$ order system

$$
\text { Equation 3-9 } \quad H(s)=\frac{\omega_{n}^{2}}{s^{2}+2 \xi \omega_{n} s+\omega_{n}^{2}},
$$

the impulse response of this system is an exponentially damped sinusoid, which can be described as,

$$
\text { Equation 3-10 } h(t)=A e^{-\xi \omega_{n} t} \sin \left(\omega_{n} \sqrt{1-\xi^{2} t}\right),
$$

where $A$ is the residue, $\xi$ is the damping ratio, and $\omega_{n}$ is the resonance frequency of this system. The envelope of $h(t)$ is the magnitude of the pre-envelope signal $C(t)$ [64],

$$
\text { Equation 3-11 } \quad C(t)=h(t)+i \hat{h}(t),
$$

where $x(t)$ is the impulse response, and $\hat{x}(t)$ is the Hilbert transform of $h x(t)$. Since $h(t)$ is exponentially damped, the natural logarithm of $|C(t)|$ is a line, and the damping ratio can be estimated by Equation 3-12. This will be proven later. 
Equation 3-12 $\quad \xi=\frac{-\ln |C(t)|}{\omega_{n}}$.

The Hilbert transform of a signal is defined as a signal with $-\frac{\pi}{2}$ radians' phase shift of all frequency components of the original signal. The Hilbert transform of this impulse response signal described in Equation 3-10 is:

$$
\text { Equation 3-13 } \quad \hat{h}(t)=A e^{-\xi \omega_{n} t} \cos \left(\omega_{n} \sqrt{1-\xi^{2} t}\right),
$$

Then the combined signal will be:

$$
\text { Equation 3-14 } C(t)=A e^{-\xi \omega_{n} t}\left(\sin \left(\omega_{n} \sqrt{1-\xi^{2}} t\right)+i \cos \left(\omega_{n} \sqrt{1-\xi^{2}} t\right)\right),
$$

The magnitude of $C(t)$ is:

$$
\text { Equation 3-15 }|C(t)|=A e^{-\xi \omega_{n} t} \sqrt{\sin ^{2}\left(\omega_{n} \sqrt{1-\xi^{2}} t\right)+\cos ^{2}\left(\omega_{n} \sqrt{1-\xi^{2}} t\right)}=A e^{-\xi \omega_{n} t},
$$

By taking natural logarithm on both side of Equation 3-15,

$$
\text { Equation 3-16 } \quad \ln (|C(t)|)=\ln (A)-\xi \omega_{n} t,
$$

The damping ratio can be deduced

$$
\text { Equation 3-17 } \xi=\frac{\frac{\ln (|C(t)|-\ln (A))}{t}}{\omega_{n}}=\frac{- \text { slope }}{\omega_{n}} .
$$

For the lateral comb drive resonator involved in this study, the transfer function is shown in Equation 3-3, rewriting in the format of Equation 3-9 results in

$$
\text { Equation 3-18 } \quad \beta=2 m \xi \omega_{n},
$$

where the effective mass $m$ can be calculated by Equation 3-6, and the resonance frequency $\omega_{n}$ can be estimated by counting the period of the system's impulse response. Even though the theoretical calculation of the effective mass could not be exactly the same as the actual mass of the system due to the fabrication tolerance, effective mass is still the most robust parameter regarding to the manufacturing tolerance (Table 3-2). Even for the worst case, the error is less than $1.8 \%$ for the $450 \mu \mathrm{m}$ fracture length device. 
Therefore the use of calculated mass in this equation won't lead too much error in the estimation of the damping coefficient.

The performance of this method has been evaluated by simulation, using an LCR with parameters of $m=3.3124 \times 10^{-10} \mathrm{~g}, \beta=1.0101 \times 10^{-6}$, and $k_{s}=0.034164$ simulated using with MATLAB simulink model. The impulse response of this LCR model looks like the one shown in Figure 3-4, and the natural logarithm of its envelope is just a straight line as shown in Figure 3-5.

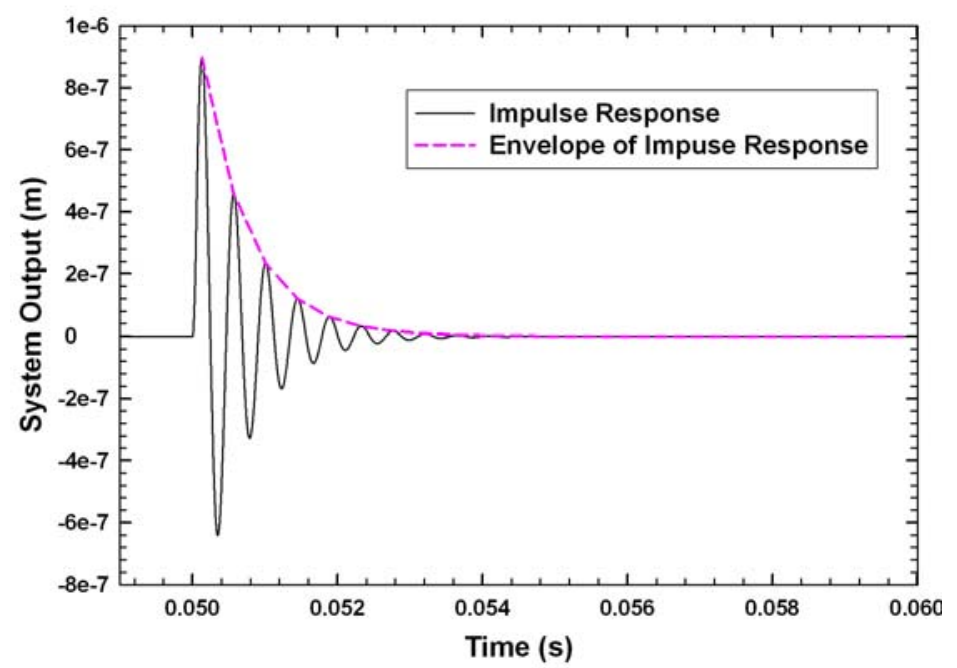

Figure 3-4: $\quad$ Impulse Response of a Lateral comb Resonator

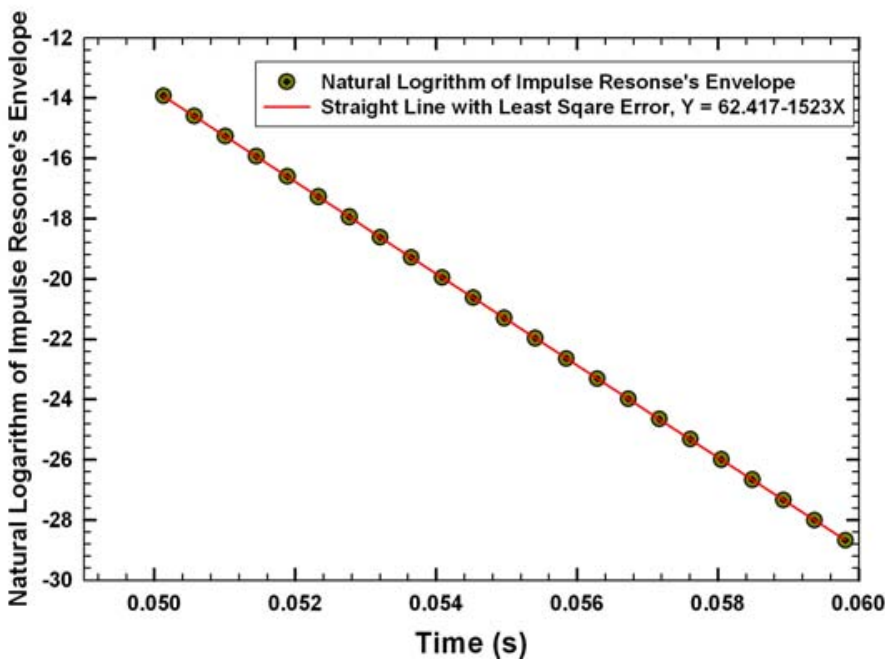

Figure 3-5: $\quad$ Natural Logarithm of the Impulse Response's Envelope 
Genetic Algorithm has been applied to find constants $a$ and $b$, which can make the minimum square error between the logarithm of the impulse response's envelop and line $y=a+b t$. The $b$ found in this way will be treated as the slope in Equation 3-17. In this particular example, the least square error can be reached when $a=62.417$, and $b=-1523$. The resonance frequency of this system can be estimated by counting the time interval between each peak of the system's impulse response, and it is $2.2727 \mathrm{k} \mathrm{Hz}$ in this case. To consider the worst case that the mass for fabricated LCR can be $1.8 \%$ less than the designed value due to the fabrication tolerance, instead of using $3.3124 \mathrm{e}-10 \mathrm{~kg}$ as the theoretical mass, we use $3.3123 \times 10^{-10} \div 101.8 \%=3.2538 \times 10^{-10} \mathrm{~kg}$. Then with Equation 3-17 and Equation 3-18, the damping can be estimated as:

$$
\hat{\beta}=2 m \frac{- \text { slop }}{\omega_{n}} \omega_{n}=-2 m \cdot b=2 \cdot 3.2538 \times 10^{-10} \cdot 1523=9.911 \times 10^{-7} .
$$

Similarly, with Equation 3-5 we get:

$$
\hat{k}_{s}=\frac{\omega_{n}^{2} m}{2}=\frac{(2 \pi \cdot 2272.7)^{2} \cdot 3.2538 \times 10^{-10}}{2}=0.0331745 \text {. }
$$

The relative error between the estimation values and the real parameters will be

$$
\begin{aligned}
& E_{m}=\frac{|m-\hat{m}|}{m}=\frac{|3.3124-3.2538|}{3.3124}=0.0177, \\
& E_{\beta}=\frac{|\beta-\hat{\beta}|}{\beta}=\frac{|1.0101-0.9911|}{1.0101}=0.01881, \text { and } \\
& E_{k_{s}}=\frac{\left|k_{s}-\hat{k}_{s}\right|}{k_{s}}=\frac{|0.034164-0.0331745|}{0.034164}=0.0289 .
\end{aligned}
$$

\subsubsection{Frequency Sweeping Method}

For a stable continuous-time linear time invariant (LTI) system, the input-output behavior can be completely described by its transfer function, $H(j \omega)$ [65]. When the input applied to a system is sinusoidal

$$
\text { Equation 3-19 } u(t)=a \sin \left(\omega_{0} t\right)
$$

the steady state output of the system will be sinusoid too, and it can be described as 
Equation 3-20 $y(t)=a\left|H\left(j \omega_{0}\right)\right| \sin \left(\omega_{0} t+\angle H\left(j \omega_{0}\right)\right)$.

If the noise is negligible, the transfer function at $\omega_{0}$ can be represented as:

Equation 3-21 $H\left(j \omega_{0}\right)=\left|H\left(j \omega_{0}\right)\right| e^{j \angle H\left(j \omega_{0}\right)}$.

With Equation 3-4 (LCR's transfer function), the gain and phase shift of the system can be calculated as

$$
\begin{array}{cc}
\text { Equation 3-22 } & |H(\omega)|=\frac{1 / m}{\sqrt{\left(\frac{2 k_{s}}{m}-\omega\right)^{2}+\left(\frac{\beta}{m} \omega\right)^{2}}} \\
\text { Equation 3-23 } & \angle H(j \omega)=\tan ^{-1}\left(-\frac{j \frac{\beta}{m} \omega}{\frac{2 k_{s}}{m}-\omega^{2}}\right)
\end{array}
$$

By driving the system with a sinusoidal inputs at different frequencies, which is essentially doing a frequency sweep (frequencies across the resonance) on the system, a set of experimental gains and phase shifts can be obtained. A Genetic Algorithm has been applied again to find $m, \beta$, and $k_{s}$ which can best match the theoretical curve of gain and phase to the experimental curve (Figure 3-6). All the parameters that are involved in this system can be estimated directly. Simulation with the same system that was used in the Hilbert envelope method has been performed, and this time, the relative errors of the parameter estimation are

$$
\begin{aligned}
& E_{m}=\frac{|m-\hat{m}|}{m}=\frac{|3.3124-3.3262|}{3.3124}=0.00416 \\
& E_{\beta}=\frac{|\beta-\hat{\beta}|}{\beta}=\frac{|1.0101-1.0083|}{1.0101}=0.00181, \text { and } \\
& E_{k s}=\frac{\left|k_{s}-\hat{k}_{s}\right|}{k_{s}}=\frac{|0.034164-0.03423|}{0.034164}=0.0019245
\end{aligned}
$$



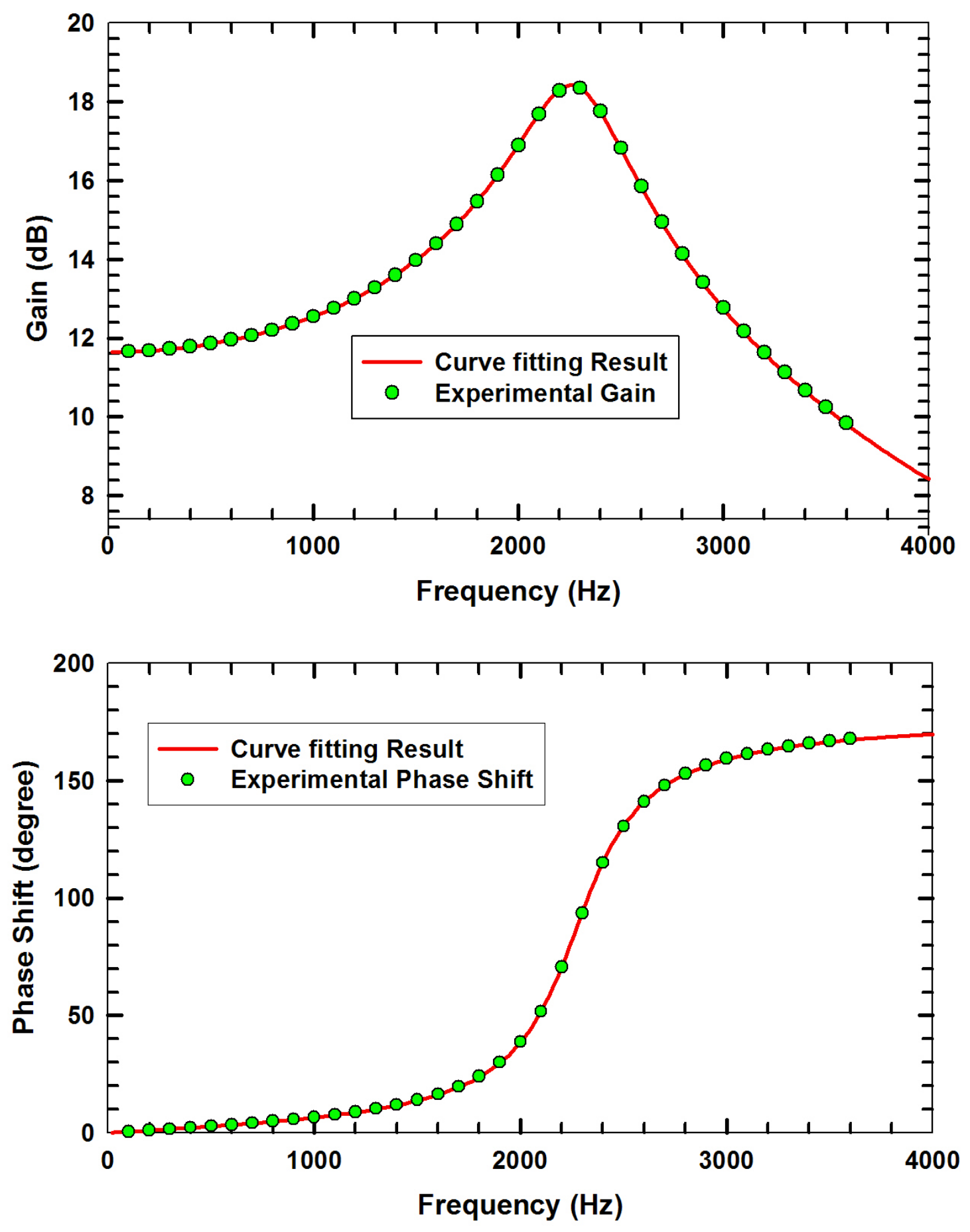

Figure 3-6 Curve fitting results of gain and phase shift plot 


\subsubsection{Frequency Domain System Identification}

The major shortcoming of time domain system identification is that high time domain accuracy of the curve matching is not well reflected in frequency domain accuracy, which may lead to large errors in the frequency domain near anti-resonance [ 66 ]. Many current researches have focused on frequency domain procedures $[67,68,69,70,71,72]$. There are many existing frequency domain methods and most of them are practically useful. A simple but effective frequency domain system identification approach has been presented in this study.

For the $2^{\text {nd }}$ order MEMS lateral comb resonator system (Equation 3-3), the difference of system parameter(s) will result in difference in the power spectrum of its output (Figure 3-7) when the inputs to the system are the same. The first step of this approach is to perform an FFT on the system output, then the power spectrum of output signal has been used as the criterion to perform the system identification. As shown in Figure 3-7, even with same inputs, there will be a difference in the power spectrum of system outputs when the parameters involved in the system have minor changes. By comparing the experimental output's power spectrum with those obtained through simulation, the system parameters can be finally identified. A Genetic Algorithm has also been applied in this method to figure out parameters that are involved in the transfer function. The idea is to match the power spectrum of the simulation output with the power spectrum of the experimental output by adjusting the parameters of the simulation model with a GA method.

A simulation has been performed to evaluate the performance of this frequency domain parameter identification method. The same system that used in the previous time domain methods has been used. By exciting the system with random multisine force, the power spectrum of the system output will look like the circles in Figure 3-8. 


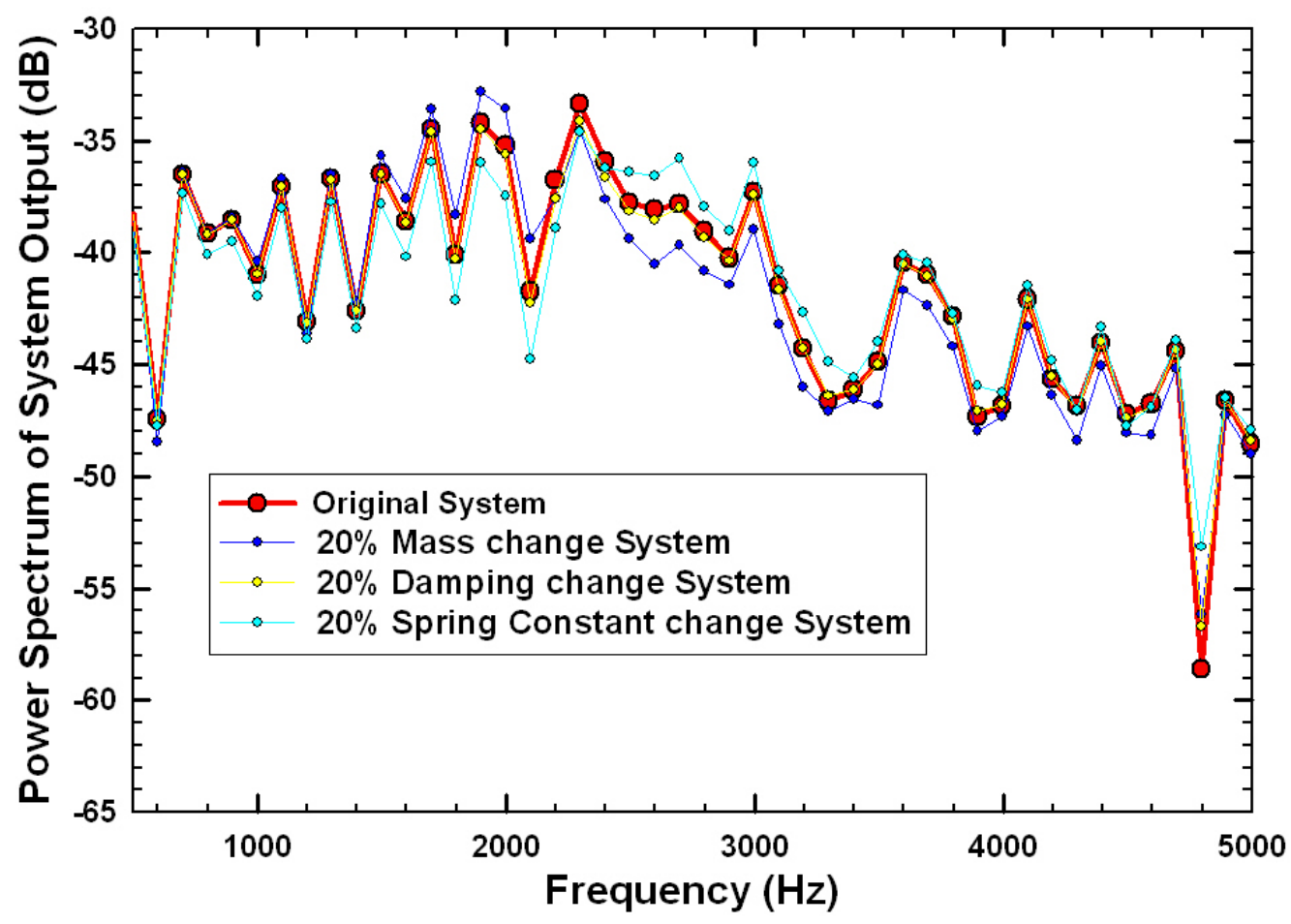

Figure 3-7: $\quad$ Simulated Frequency Response for MEMS Lateral COMB Resonator

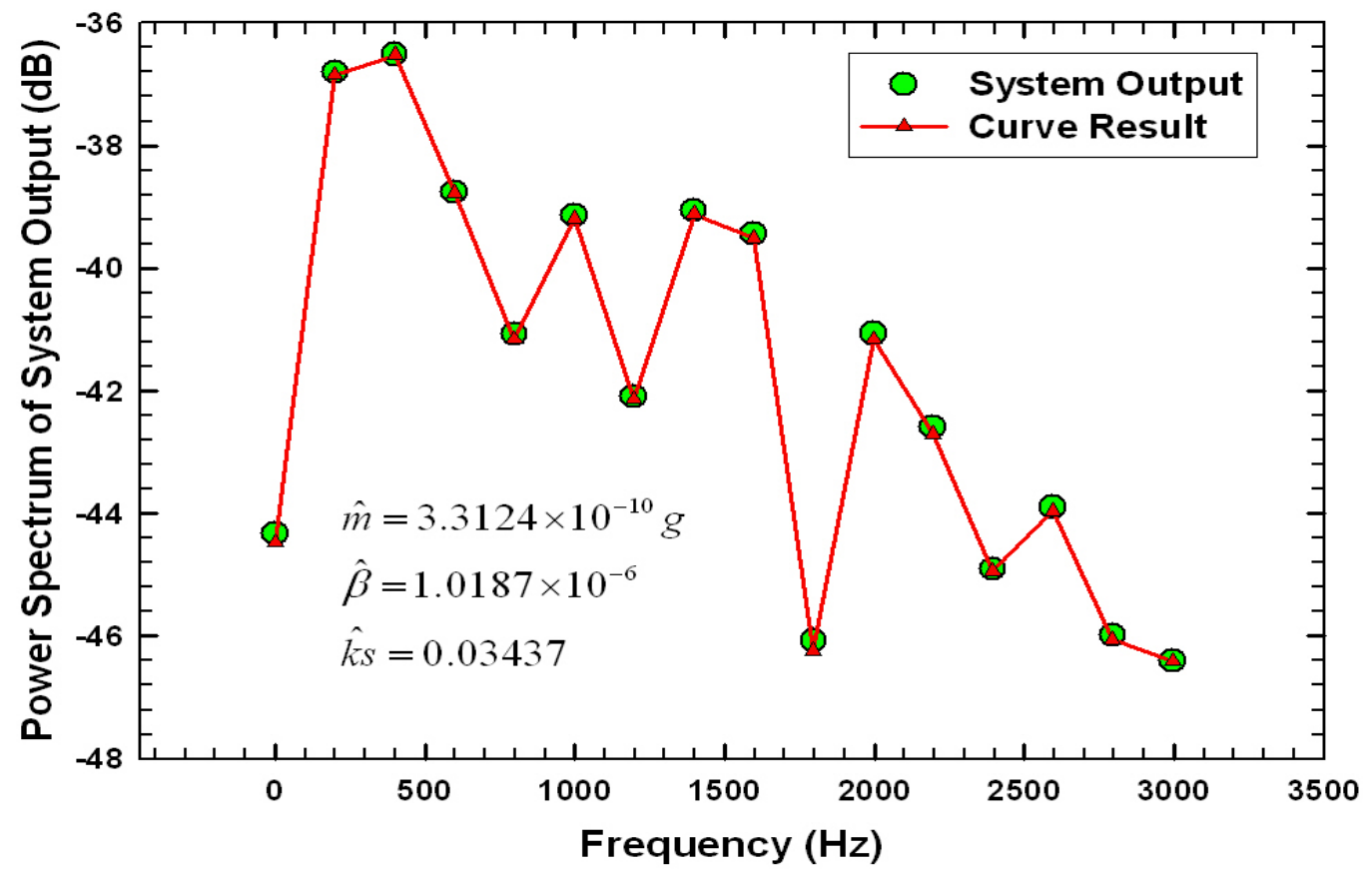

Figure 3-8: $\quad$ Parameter Estimation Result for the given system 
By performing curve fitting with the Genetic Algorithm method that was used in section 3.2.1.2, the parameters in the system can be estimated as $\hat{m}=3.3124 \times 10^{-10} \mathrm{~g}$, $\hat{\beta}=1.0187 \times 10^{-6}$, and $\hat{k} s=0.034437$. This time, the relative errors for the parameter estimation are

$$
\begin{aligned}
& E_{m}=\frac{|m-\hat{m}|}{m}=\frac{|3.3124-3.3124|}{3.3124}=0, \\
& E_{\beta}=\frac{|\beta-\hat{\beta}|}{\beta}=\frac{|1.0101-1.0187|}{1.0101}=0.00855, \text { and } \\
& E_{k_{s}}=\frac{\left|k_{s}-\hat{k}_{s}\right|}{k_{s}}=\frac{|0.034164-0.03437|}{0.034164}=0.00601 .
\end{aligned}
$$

\subsubsection{Conclusion}

From Table 3-3, we can easily find out that the frequency sweeping method has the best estimation performance among the 3 candidates, and this method has been chosen to perform the system identification in this study.

Even though the Hilbert Envelope method cannot estimate all the parameters that are involved in the system model, it is also a good candidate for those applications that need on-line system identification. Since this method is straight forward, it can be implemented on-line.

Table 3-3: Relative errors of the parameter estimation with different approaches

\begin{tabular}{|l|c|c|c|c|}
\hline \multicolumn{1}{|c|}{ Method } & $E_{m}=\frac{|m-\hat{m}|}{m}$ & $E_{k_{s}}=\frac{\left|k_{s}-\hat{k}_{s}\right|}{k_{s}}$ & $E_{\beta}=\frac{|\beta-\hat{\beta}|}{\beta}$ & $\begin{array}{c}\text { Total Error } \\
E_{m}+E_{k s}+E_{\beta}\end{array}$ \\
\hline Hilbert Envelope & $0.0177^{*}$ & 0.01881 & 0.0289 & 0.06547 \\
\hline Frequency Sweeping & 0.00416 & 0.0019245 & 0.00181 & 0.007895 \\
\hline Frequency Domain & 0 & 0.00601 & 0.00855 & 0.01456 \\
\hline
\end{tabular}

Compared with the time domain's frequency sweeping method, although the frequency domain method presents more errors in its estimation results, it requires only one set of experimental data to be recorded to get good estimation results, while the other

\footnotetext{
* This parameter cannot be estimated with this method, and this value here is based on the worst assumption.
} 
method requires at least 4 sets of data to be recorded. Therefore, this method can also be considered as a good candidate for system identification in this study.

\subsection{Experimental Implementation of Parameter Identification}

All the three system identification methods have been experimentally implemented in this study. Both time domain methods and the frequency domain methods have been experimentally implemented on a single open LCR, while only the frequency sweeping method and frequency domain method have been implemented on a grating structure LCR. The results will be compared and discussed later.

\subsubsection{Experimental system identification on single opening device}

\subsubsection{Experimental implementation of Hilbert envelop method}

It is easy to understand from Equation 3-18, that the estimation of $\beta$ is based on the estimation of $\xi$, and, which the $\xi$ can be estimated by the Hilbert envelope method described above. The impulse response experiment (Figure 3-9) has been repeated eight times, and eight sets of impulse-response data have been recorded.

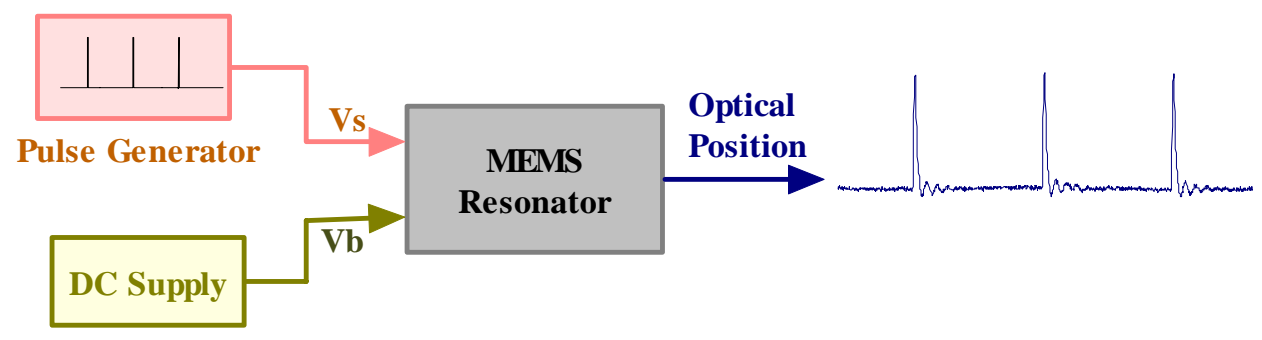

Figure 3-9 Block diagram of Impulse response experiment

The optical monitoring signals for the impulse response (Figure 3-10) were recorded by an oscilloscope. Before implementing the Hilbert envelope method, waveletdenoising method that was described in chapter 2 has been applied on these optical outputs. 

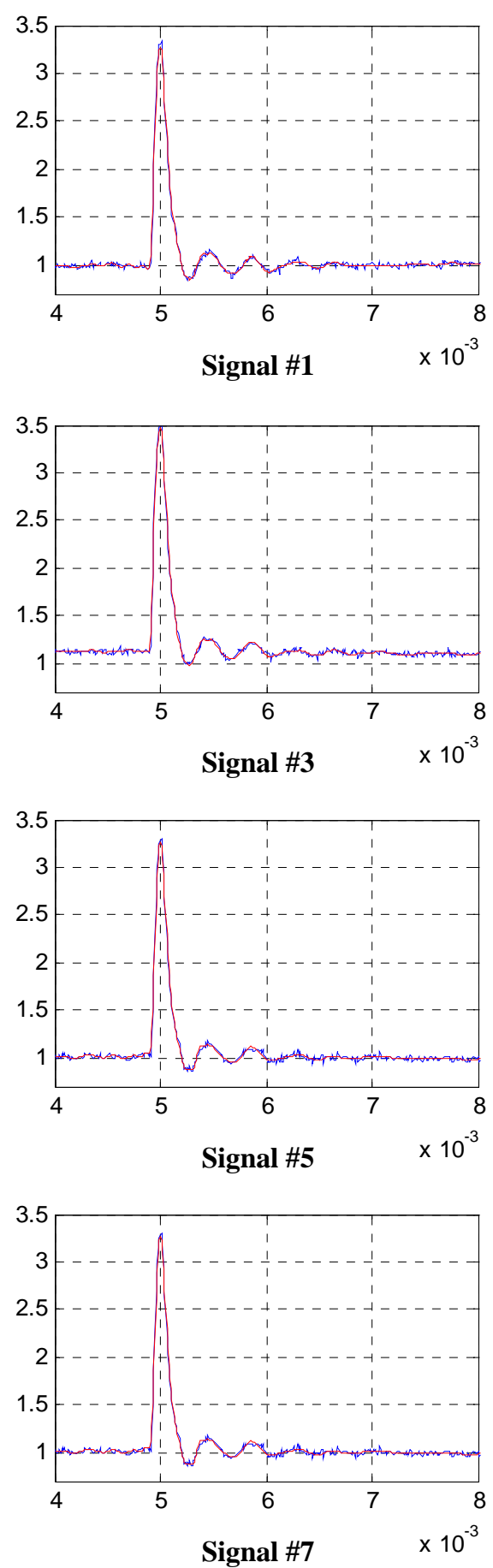
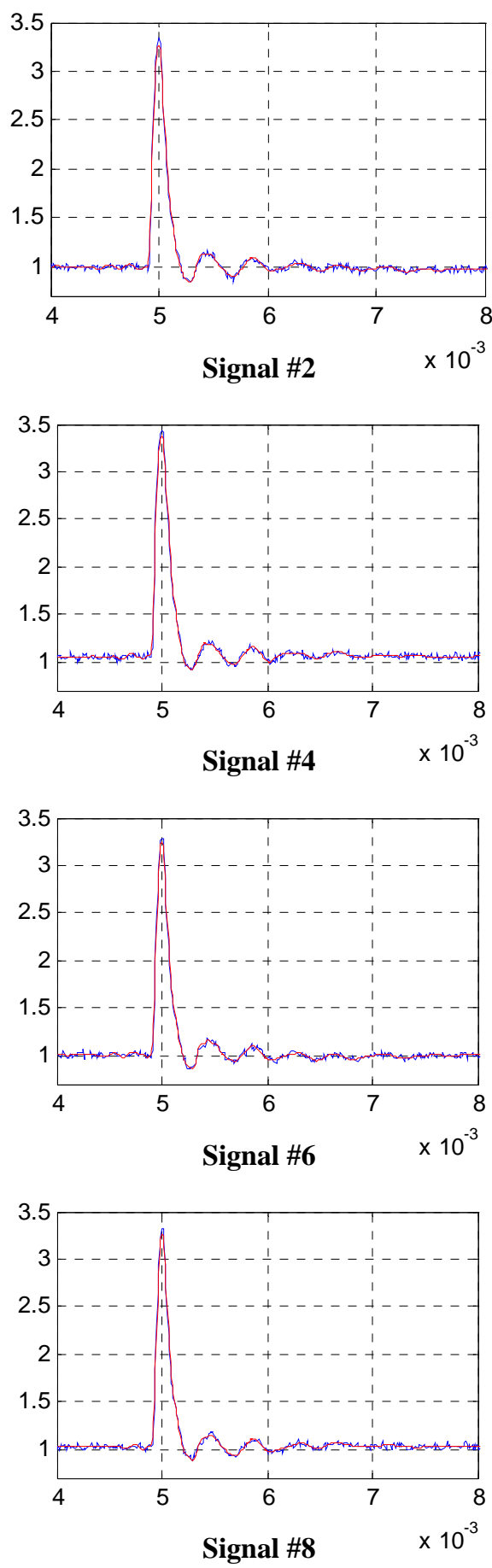

Signal \#8

Figure 3-10 Wavelet Denoising for Optical Pulse Response

The resonance frequency of the LCR has been estimated with the same method that we used in the simulation, and the estimation results for the 8 signal records are listed in Table 3-4. 
Table 3-4: Natural Frequency of the Poly2 Structure MEMS Resonator (Beam Length=450 $\mu \mathrm{m}$ )

\begin{tabular}{|c|c|c|c|c|c|c|c|c|c|}
\hline Signal & $\# \mathbf{1}$ & $\mathbf{\# 2}$ & $\mathbf{\# 3}$ & $\mathbf{\# 4}$ & $\mathbf{\# 5}$ & $\mathbf{\# 6}$ & $\mathbf{\# 7}$ & \#8 & mean \\
\hline $\begin{array}{l}\text { Resonance } \\
\text { Frequency }\end{array}$ & $2452 \mathrm{~Hz}$ & $2462 \mathrm{~Hz}$ & $2470 \mathrm{~Hz}$ & $2455 \mathrm{~Hz}$ & $2462 \mathrm{hz}$ & $2480 \mathrm{~Hz}$ & $2480 \mathrm{~Hz}$ & $2465 \mathrm{~Hz}$ & $2466 \mathrm{~Hz}$ \\
\hline
\end{tabular}

The mean of the resonance frequency based on the eight tests is

$$
f_{\text {mean }}=2466 \mathrm{~Hz} \text {, }
$$

the standard deviation of the resonance frequency is

$$
f_{\text {std }}=10.44 \mathrm{~Hz}
$$

and,

$$
f_{\text {std }} / f_{\text {mean }}=0.0042342<0.05 \text {. }
$$

Therefore, from a statistical point of view, these test results are unanimous and $2466 \mathrm{~Hz}$ can be treated as the resonance frequency of this device.

The damping ratio for this system was obtained by the same method as that used in the simulation, and the results are listed in Table 3-5.

Table 3-5: Damping Ratio of the poly2 Structure MEMS Resonator (Beam Length=450 $\mu \mathrm{m}$ )

\begin{tabular}{|c|c|c|c|c|c|c|c|c|c|}
\hline Signal & $\# \mathbf{1}$ & \#2 & \#3 & \#4 & \#5 & \#6 & \#7 & \#8 & mean \\
\hline$\xi$ & 0.074447 & 0.077239 & 0.079094 & 0.079438 & 0.080414 & 0.081891 & 0.080515 & 0.079093 & 0.079016 \\
\hline
\end{tabular}

The mean of $\xi$ is

$$
\xi_{\text {mean }}=0.079016 \text {, }
$$

the standard deviation of $\xi$ is

$$
\xi_{\text {std }}=0.022895 \text {, }
$$

and,

$$
\xi_{\text {std }} / \xi_{\text {mean }}=0.028975<0.05
$$

Again, from a statistical point of view, we can accept 0.079016 as the Damping ratio for this system.

Based on the assumption that the theoretical calculated effective mass $\left(2.1249 \times 10^{-10} \mathrm{~kg}\right)$ is very close to the actual, with Equation $3-18$, the damping of the system can be estimated as:

$$
\beta=5.2024 \times 10^{-7},
$$

and the spring constant can be estimated with 


$$
\text { Equation 3-24 } \quad k_{s}=\frac{m \omega_{n}^{2}}{2}=2 \pi^{2} m f_{n}^{2} \text {. }
$$

Therefore spring constant $k_{s}$ will be

$$
k_{s}=2 \pi^{2}\left(2.1249 \times 10^{-10}\right)(2466)^{2}=0.0255 .
$$

Compared with the theoretical spring constant 0.0252, this result is reasonable. That, in turn, means the damping factor we estimated here is also reasonable.

\subsubsection{Experimental implementation of frequency sweeping method}

In the experimental implementation, sine waves with frequency ranging from $100 \mathrm{~Hz}$ to $3000 \mathrm{~Hz}$ were selected as inputs to the system, and the corresponding outputs of this system have been recorded by an oscilloscope. Since the outputs are sort of noisy, a wavelet-denoising step has to be taken before the system identification can be performed (chapter 2).

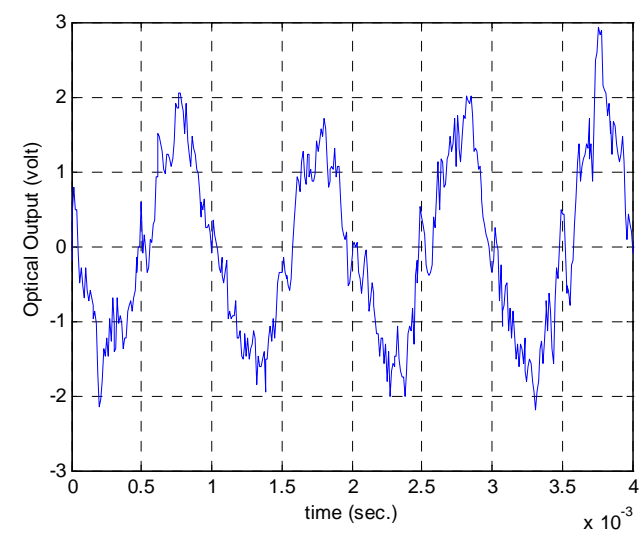

(a)

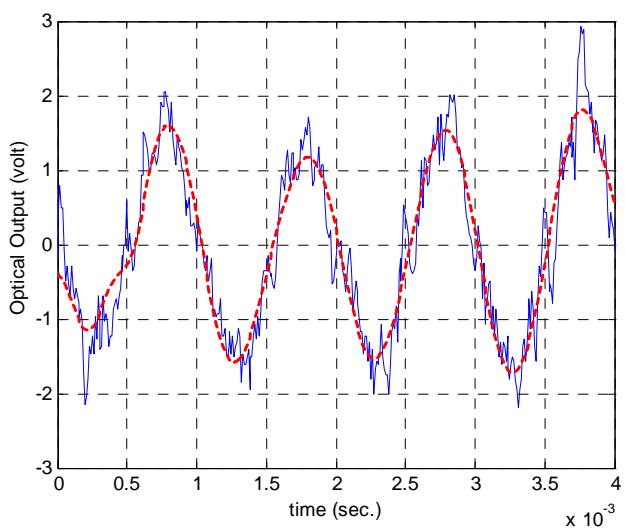

(b)

Figure 3-11 Wavelet-denoising on output signal, ---(a) orignial signal, (b) original signal and denoised signal

A signal at $1000 \mathrm{~Hz}$ (Figure 3-12) has been chosen as an example to illustrate the way that the gain and phase shift are to be calculated for experimental data. 


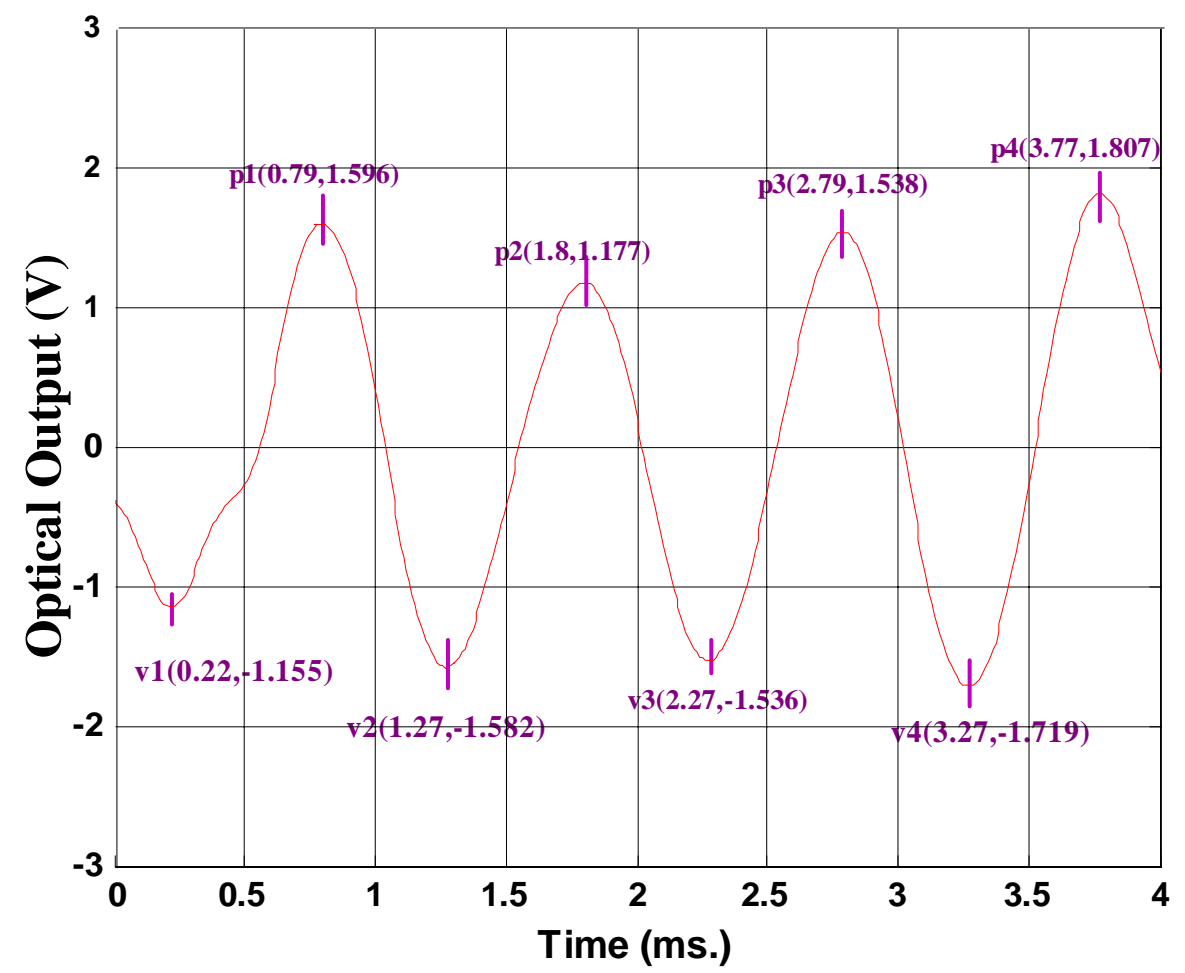

Figure 3-12 Noise reduced Optical Output of MEMS Resonator, with force at $1000 \mathrm{~Hz}$

To calculate the gain, the amplitude of the output has to be determined first. It is easy to understand:

Equation 3-25 $\quad A_{o p 1000}=\frac{\left(p_{1}-v_{1}\right)+\left(p_{2}-v_{2}\right)+\left(p_{3}-v_{3}\right)+\left(p_{4}-v_{4}\right)}{8}=1.51375$

where:

$$
\begin{aligned}
& A_{\text {op1000 }} \text { : amplitude of optical output at } 1000 \mathrm{~Hz}, \\
& p_{i} \text { : peak value of optical signals (Figure 3-12), } \\
& v_{i} \text { : valley value of optical signals (Figure 3-12). }
\end{aligned}
$$

Therefore, with the relationship that one volt in optical signal represents $\mathrm{s} \mu \mathrm{m}$ in position output, the amplitude of position output at $1000 \mathrm{~Hz}$ will be

$$
A_{1000}=1.51375 \times s=1.51375 s(\mu \mathrm{m}),
$$


where $s$ is the scale factor between optical signal and position signal. In other words, we assumed that

$$
\text { poistion_signal =optical_signal } \times \text { s. }
$$

Since $V_{B}=10 v$, and $\left|V_{S}\right|=10 v$, from Equation 3-2, we got

$$
\left|F_{e}\right|=1.2693 e \times 10^{-7} \mathrm{~N}
$$

Then the gain at $1000 \mathrm{~Hz}$,

$$
G_{1000}=10 \log _{10}\left(\frac{A_{1000}}{|F e|}\right)=\left(10.765+s^{*}\right) d b .
$$

The phase shift between the output and input can be calculated by finding the phase angle between the FFT of both signals. The program for the phase shift calculation can be found in appendix A, and the phase shift at $1000 \mathrm{~Hz}$ that was calculated using this program is 0.218. Because the position output signal is sinusoidal with the same frequency as the input, using the amplitude obtained in Equation 3-25, and the phase shift value of 0.218 , a new signal ( $\left.S_{N E W}\right)$ can be built $\left(S_{N E W}=1.51375 \sin (1000 \times 2 \pi t+0.218)\right)$. This new signal shows a good match to the original optical output (Figure 3-13).

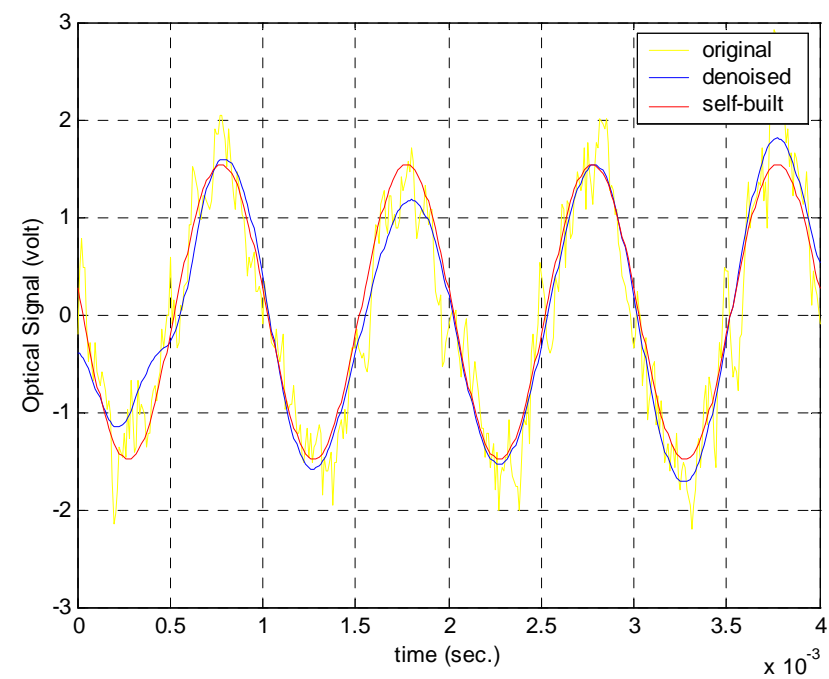

Figure 3-13: The Artificially Built New Signal which Matches the Experiment Optical Output

The Gains and Phase delays for other frequencies were calculated in the same way, and are listed in Table 3-6. 
Table 3-6 Experimental Gains and Phase Delays of the poly2 structure MEMS Resonator (Beam Length: $450 \mu \mathrm{m}$ )

\begin{tabular}{|c|c|c|c|c|c|}
\hline $\begin{array}{c}\text { Frequency } \\
\mathbf{( H z})\end{array}$ & $\begin{array}{c}\text { Gain* } \\
\text { 10log10(Position/Force) }\end{array}$ & $\begin{array}{c}\text { Phase Delay } \\
\text { (degree) }\end{array}$ & $\begin{array}{c}\text { Frequency } \\
\text { (Hz) }\end{array}$ & $\begin{array}{c}\text { Gain* } \\
\text { 10log10(Position/Force) }\end{array}$ & $\begin{array}{c}\text { Phase Delay } \\
\text { (degree) }\end{array}$ \\
\hline $\mathbf{1 0 0}$ & $5.84545+s^{*}$ & -0.862 & $\mathbf{1 6 0 0}$ & $10.62063+s^{*}$ & 0.358407 \\
\hline $\mathbf{2 0 0}$ & $8.16146+s^{*}$ & -0.292 & $\mathbf{1 7 0 0}$ & $10.77959+\mathrm{s}^{*}$ & 0.408 \\
\hline $\mathbf{3 0 0}$ & $8.36859+\mathrm{s}^{*}$ & -0.172 & $\mathbf{1 8 0 0}$ & $11.29642+\mathrm{s}^{*}$ & 0.438 \\
\hline $\mathbf{4 0 0}$ & $8.32312+\mathrm{s}^{*}$ & -0.152 & $\mathbf{1 9 0 0}$ & $11.94989+\mathrm{s}^{*}$ & 0.508 \\
\hline $\mathbf{5 0 0}$ & $8.25684+\mathrm{s}^{*}$ & -0.112 & $\mathbf{2 0 0 0}$ & $12.51903+\mathrm{s}^{*}$ & 0.678 \\
\hline $\mathbf{6 0 0}$ & $8.44633+\mathrm{s}^{*}$ & 0.008 & $\mathbf{2 1 0 0}$ & $12.85489+\mathrm{s}^{*}$ & 0.808 \\
\hline $\mathbf{7 0 0}$ & $8.2725+\mathrm{s}^{*}$ & -0.042 & $\mathbf{2 2 0 0}$ & $13.75557+\mathrm{s}^{*}$ & 0.878 \\
\hline $\mathbf{8 0 0}$ & $8.37575+\mathrm{s}^{*}$ & 0.008 & $\mathbf{2 3 0 0}$ & $14.28494+\mathrm{s}^{*}$ & 1.058 \\
\hline $\mathbf{9 0 0}$ & $8.29706+\mathrm{s}^{*}$ & 0.058 & $\mathbf{2 4 0 0}$ & $14.78079+\mathrm{s}^{*}$ & 1.308 \\
\hline $\mathbf{1 0 0 0}$ & $8.91627+\mathrm{s}^{*}$ & 0.218 & $\mathbf{2 5 0 0}$ & $14.9701+\mathrm{s}^{*}$ & 1.708 \\
\hline $\mathbf{1 1 0 0}$ & $9.29928+s^{*}$ & 0.118 & $\mathbf{2 6 0 0}$ & $15.08668+\mathrm{s}^{*}$ & 2.083 \\
\hline $\mathbf{1 2 0 0}$ & $9.25257+s^{*}$ & 0.198 & $\mathbf{2 7 0 0}$ & $14.74439+\mathrm{s}^{*}$ & 2.433 \\
\hline $\mathbf{1 3 0 0}$ & $9.43445+s^{*}$ & 0.238 & $\mathbf{2 8 0 0}$ & $14.08061+\mathrm{s}^{*}$ & 2.633 \\
\hline $\mathbf{1 4 0 0}$ & $9.84586+s^{*}$ & 0.218 & $\mathbf{2 9 0 0}$ & $13.50102+\mathrm{s}^{*}$ & 2.838 \\
\hline $\mathbf{1 5 0 0}$ & $10.3095+s^{*}$ & 0.278 & $\mathbf{3 0 0 0}$ & $12.6559+\mathrm{s}^{*}$ & 2.858 \\
\hline
\end{tabular}

By plotting the experimental gains and theoretical gain curve (obtained by doing simulation with the theoretical $m, \beta$, and $K_{s}$ in Table 3-2) on the same graph, we found that the theoretical gain curve is in some sort of difference as that of the experimental gains (Figure 3-14).

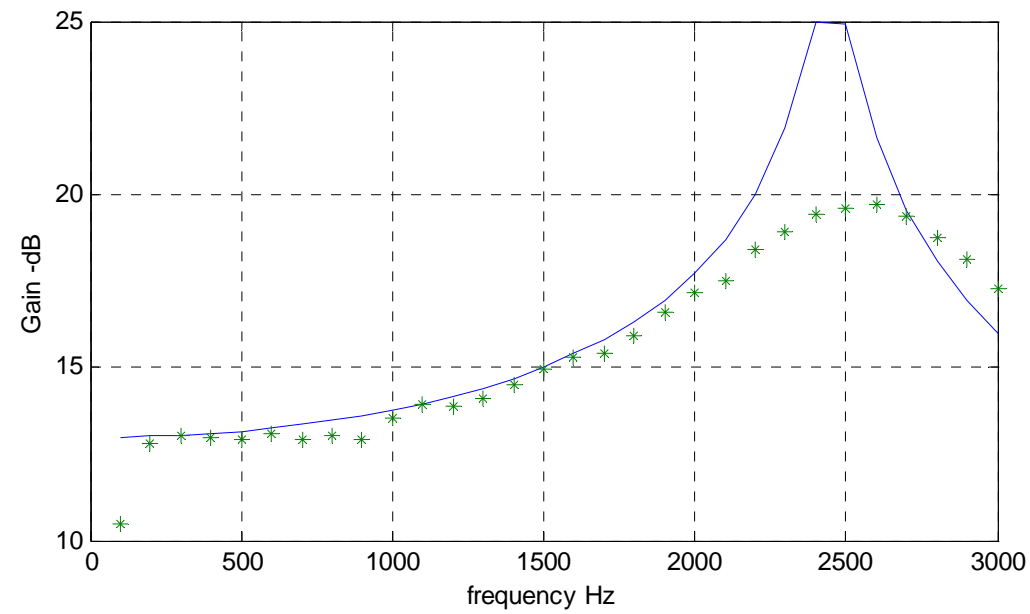

Figure 3-14 Gain plot for poly2 structure MEMS resonator with beam length $450 \mu \mathrm{m}$ (simulation result using parameters in Table 3-2, * experiment result) 

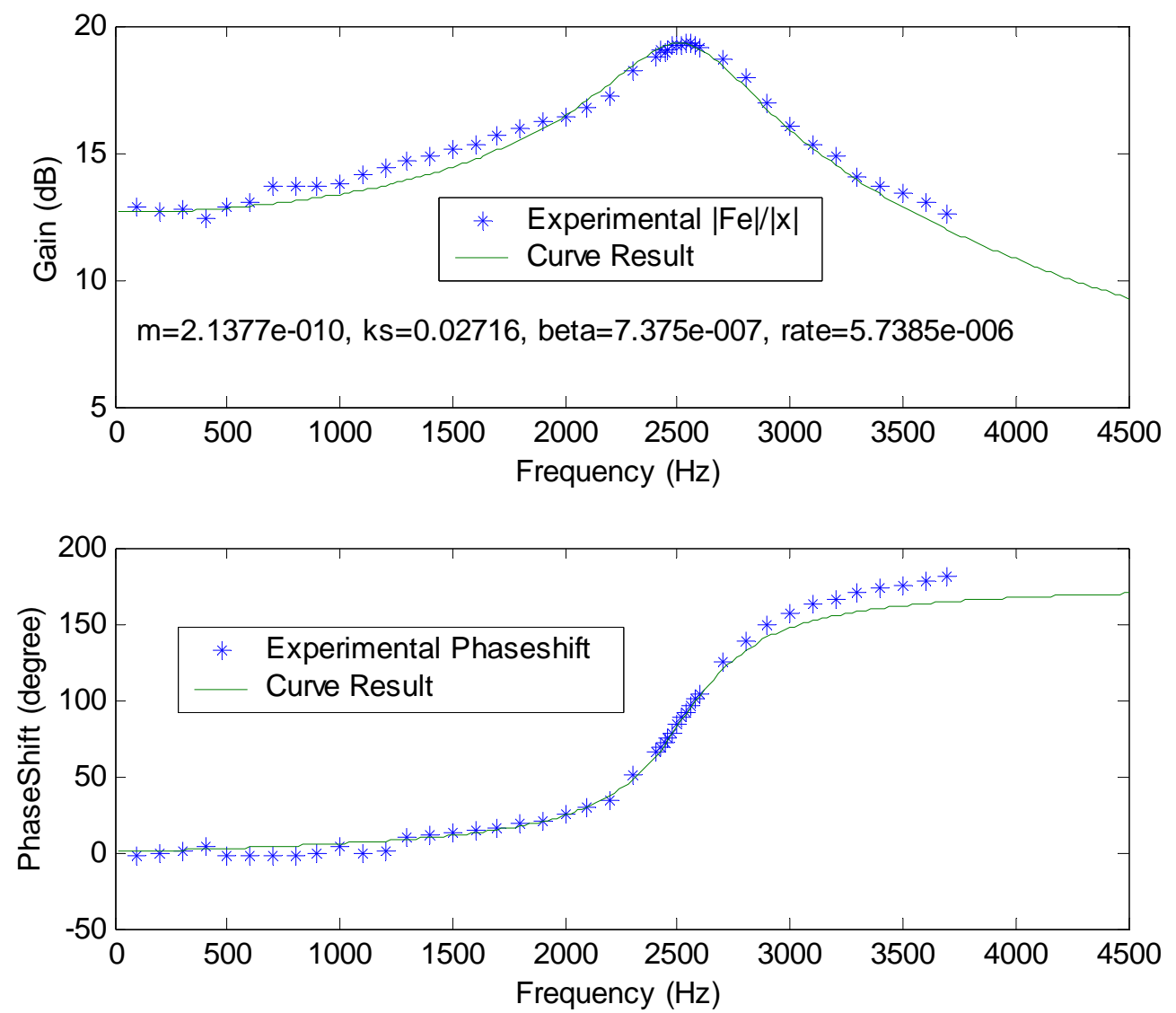

Figure 3-15 Gain plot for poly2 structure MEMS resonator with beam length $450 \mu \mathrm{m}$ (simulation result using adjusted parameters, * experiment result)

By adjusting the three parameters that are involved in the math model of the MEMS resonator and the scale factor between optical output and the position output with a Genetic Algorithm, the match between simulation gain plot and experiment gain plot is improved dramatically (Figure 3-15). This time, the parameters have been identified as:

$$
\begin{aligned}
m & =2.1377 \times 10^{-10}, \\
\beta & =7.3750 \times 10^{-7}, \\
k_{s} & =0.0272 .
\end{aligned}
$$

Even though they are close, this result is different from the identification result that we obtained with the Hilbert Envelope method. From previous analysis, that the frequency sweeping method has better reliability performance, especially compared with 
the Hilbert Envelope method. However, by carefully observing the curve fitting result of the frequency sweep method, it is easy to see that the curving lines are not matched to the experimental bode plot perfectly, and this makes the estimation result questionable. The miss match between the curving lines and bode plot shows either the gains and phase shifts we got from the optical signal are not accurate enough, or the LCR is not a perfect linear $2^{\text {nd }}$ order system.

Recalling the optical signals that we used to reveal the gain and phase information, they are pretty noisy, and the amplitudes of them are not constant. All these factors can increase the possibility that the gain and phase information we used to identify this model is not accurate enough, and might degrade the system identification result.

\subsubsection{Experimental implementation of frequency domain method}

The frequency domain method has also been performed on the same LCR device. For the voltage signal that is being applied to stage, because of the limitation of the sample rate of the hardware that we used to generate the voltage signal, instead of a random multisine signal, a multisine voltage with selected frequencies has been generated and applied to the stage in this experimental system identification. 

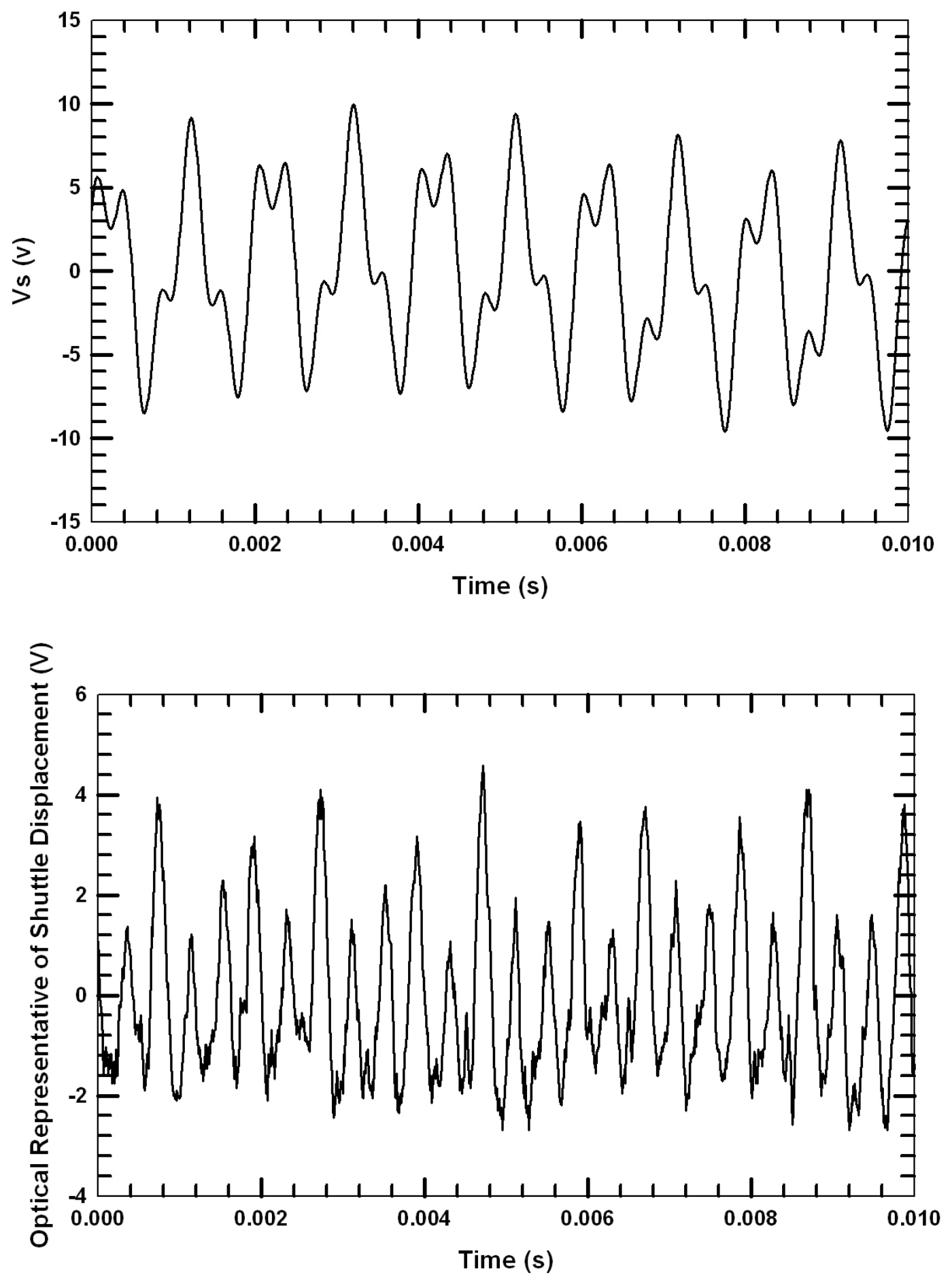

Figure 3-16 Multisine inputs to the LCR and its corresponding optical Output 


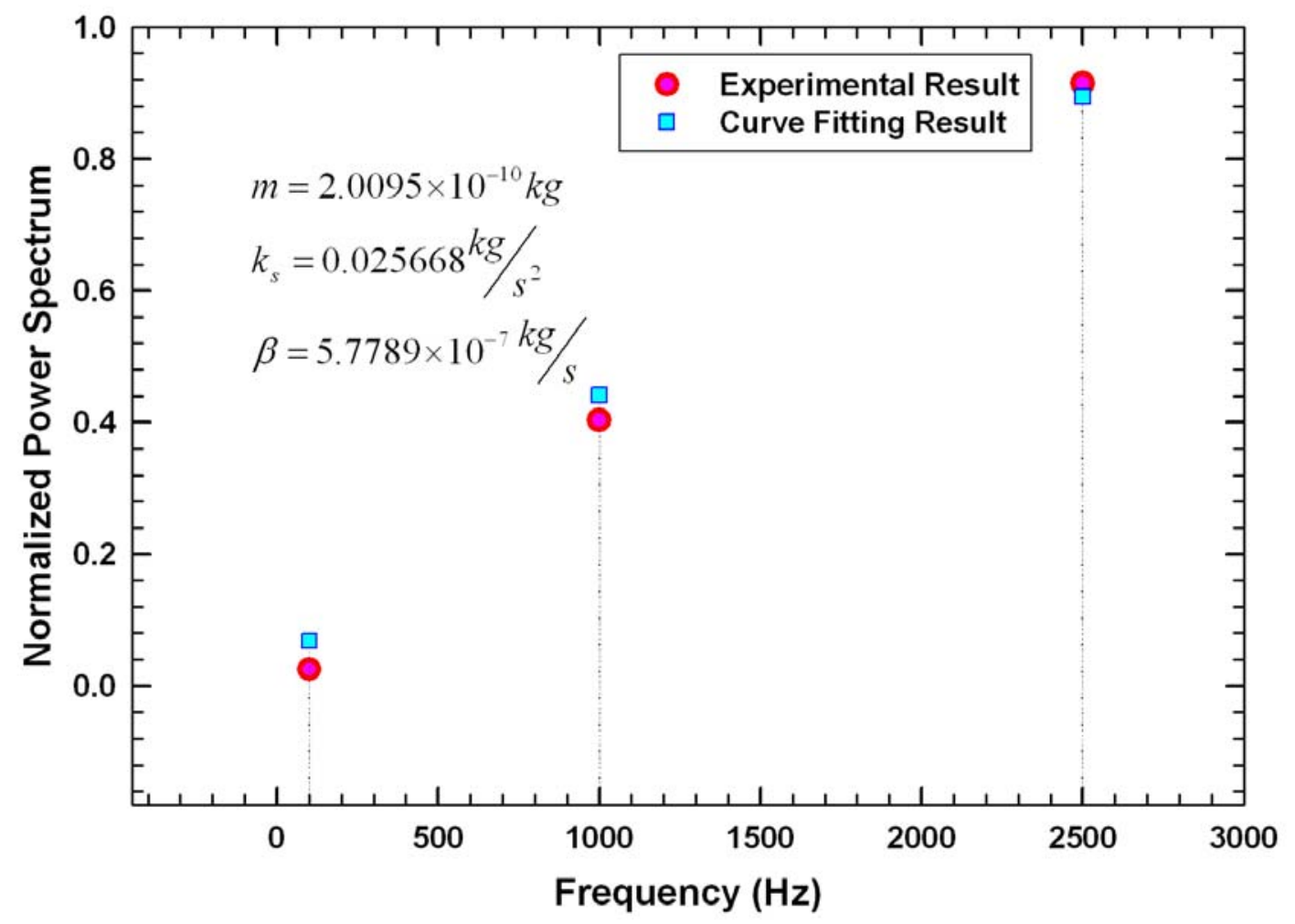

Figure 3-17 Curve fitting result for selected frequencies

Since the noise existing in the optical signal will also be reflected in its power spectrum, only three frequency components that show in input signal have been considered, they are $100 \mathrm{~Hz}, 1000 \mathrm{~Hz}$, and $2500 \mathrm{~Hz}$. By curving the normalized power spectrum that contains only the three selected frequencies, the parameters have been identified as

$$
\begin{aligned}
m & =2.00957 \times 10^{-10}, \\
\beta & =5.7789 \times 10^{-7}, \\
k_{s} & =0.0257 .
\end{aligned}
$$

The parameters estimated by all three different identification methods are listed together in Table 3-7. The estimation results for Hilbert Envelope and frequency domain method agree well with each other, and they also match with the theoretical parameters 
(see the parameter range listed in Table 3-2). Since the frequency domain method is the only method that can estimate all the parameters among these two, the parameters that are estimated with this method will be the first optimal selection for the system identification result.

Table 3-7 Length: $450 \mu \mathrm{m}$ )

\begin{tabular}{|c|c|c|c|c|}
\hline & Mass $(\mathbf{m})$ & Spring Constant $\left(\boldsymbol{k}_{\boldsymbol{s}}\right)$ & Damping $(\boldsymbol{\beta})$ & Scale Rate \\
\hline Theoretical Calculation & $2.1249 \times 10^{-10}$ & 0.0252 & - & - \\
\hline Frequency Sweep & $2.1377 \times 10^{-10}$ & 0.0272 & $7.3750 \times 10^{-7}$ & $5.7385 \mathrm{e}-6$ \\
\hline Hilbert Envelop & - & 0.0255 & $5.2030 \times 10^{-7}$ & - \\
\hline Frequency Domain & $2.00957 \times 10^{-10}$ & 0.0257 & $5.7789 \times 10^{-7}$ & - \\
\hline
\end{tabular}

In order to confirm this model, the experimental outputs at several different frequencies (cross the resonant) have been plotted together with simulation results (Figure 3-18 -- Figure 3-21). The simulations were performed by applying the same inputs as that in experiments to the system model, and using the parameters that were estimated with the frequency domain method. The experimental position outputs were obtained by multiplying the scale rate obtained from the frequency sweeping method with the optical signal. If the noises that exist in the experimental outputs are ignored, the two position signals are matching with each other perfectly in all the plots. This means the linear model we have built is acceptable for the MEMS lateral comb resonator used in this research. This confirmed model may be used to perform feedback control on the MEMS lateral comb resonator later. 


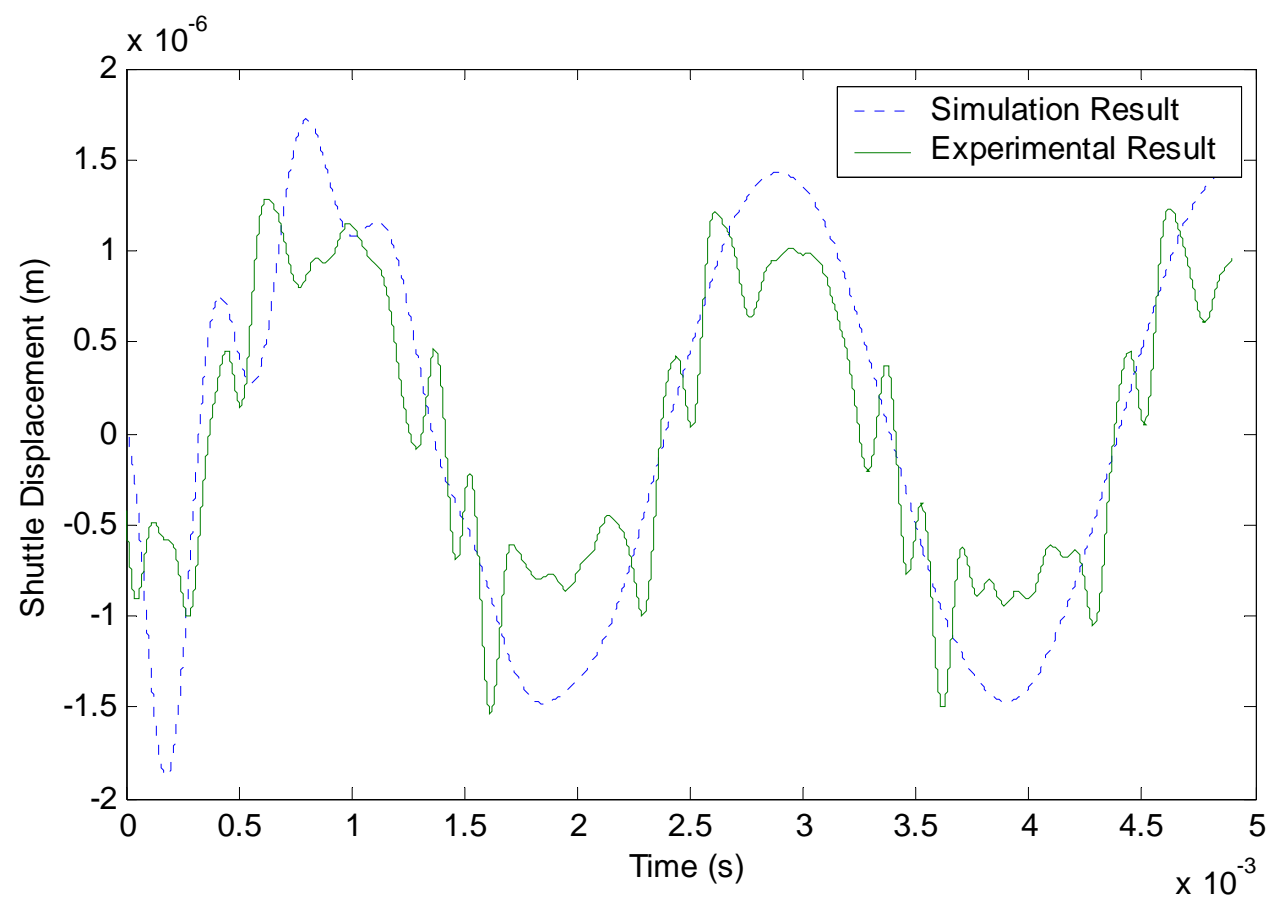

Figure 3-18 Displacement of LCR shuttle at $500 \mathrm{~Hz}$

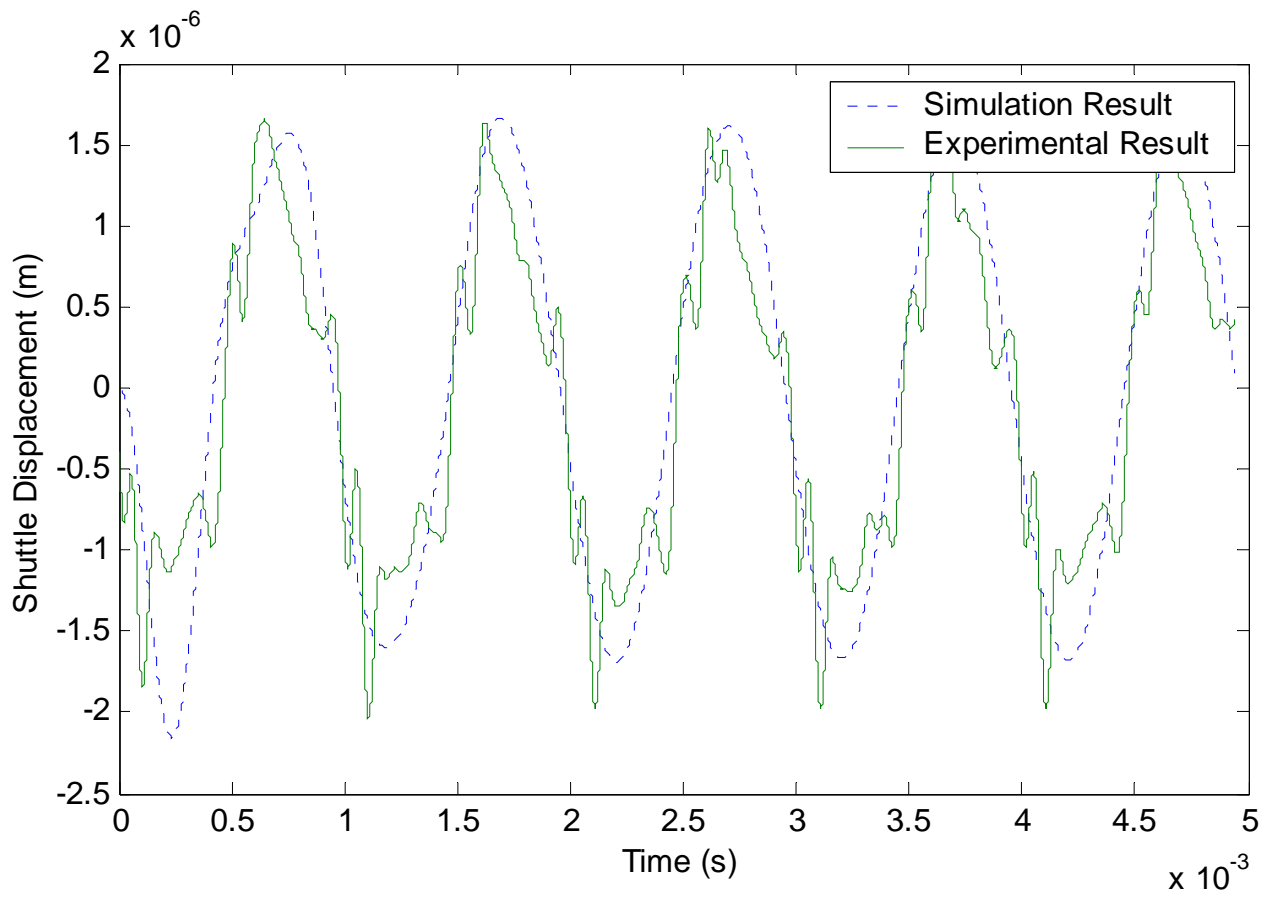

Figure 3-19 Displacement of LCR shuttle at $1000 \mathrm{~Hz}$ 


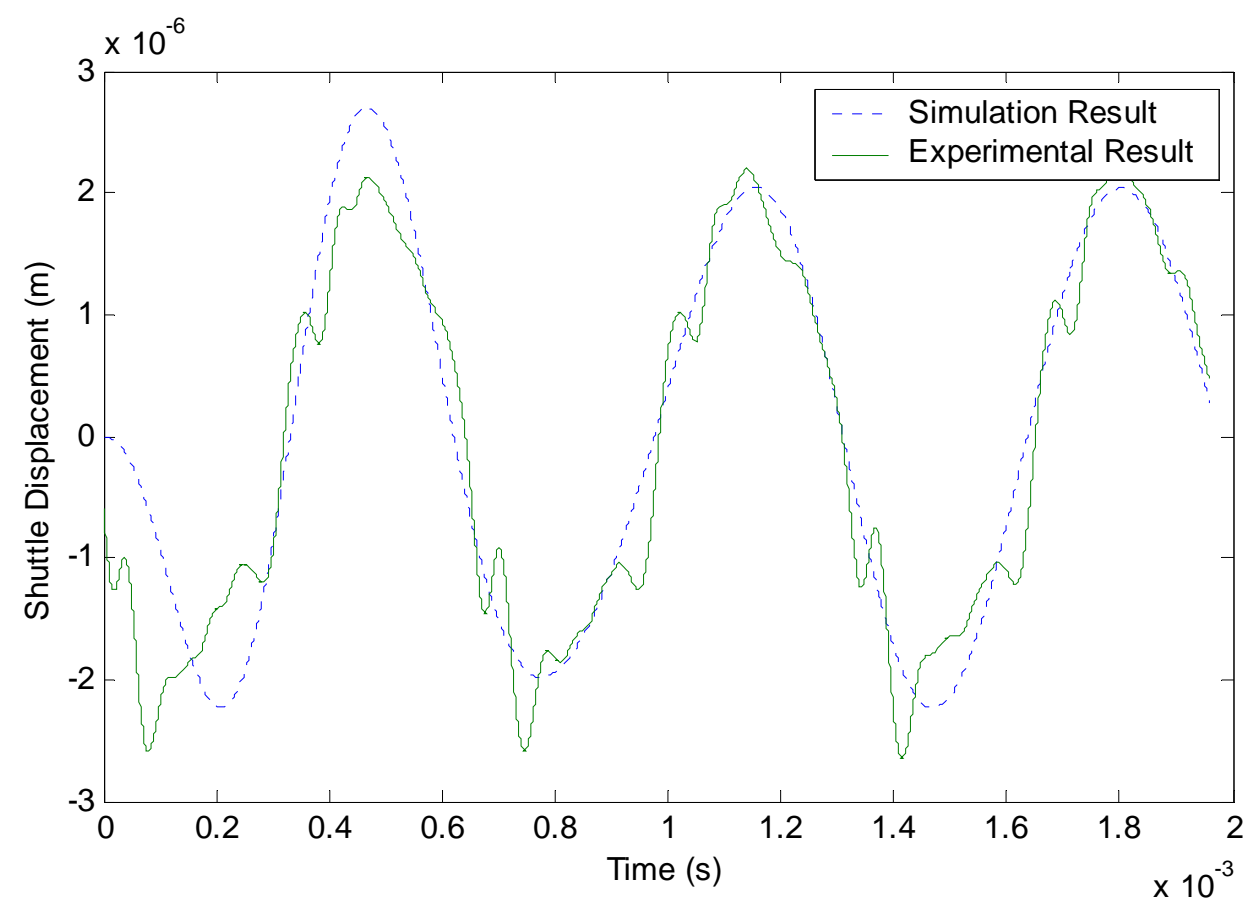

Figure 3-20 Displacement of LCR shuttle at $1500 \mathrm{~Hz}$

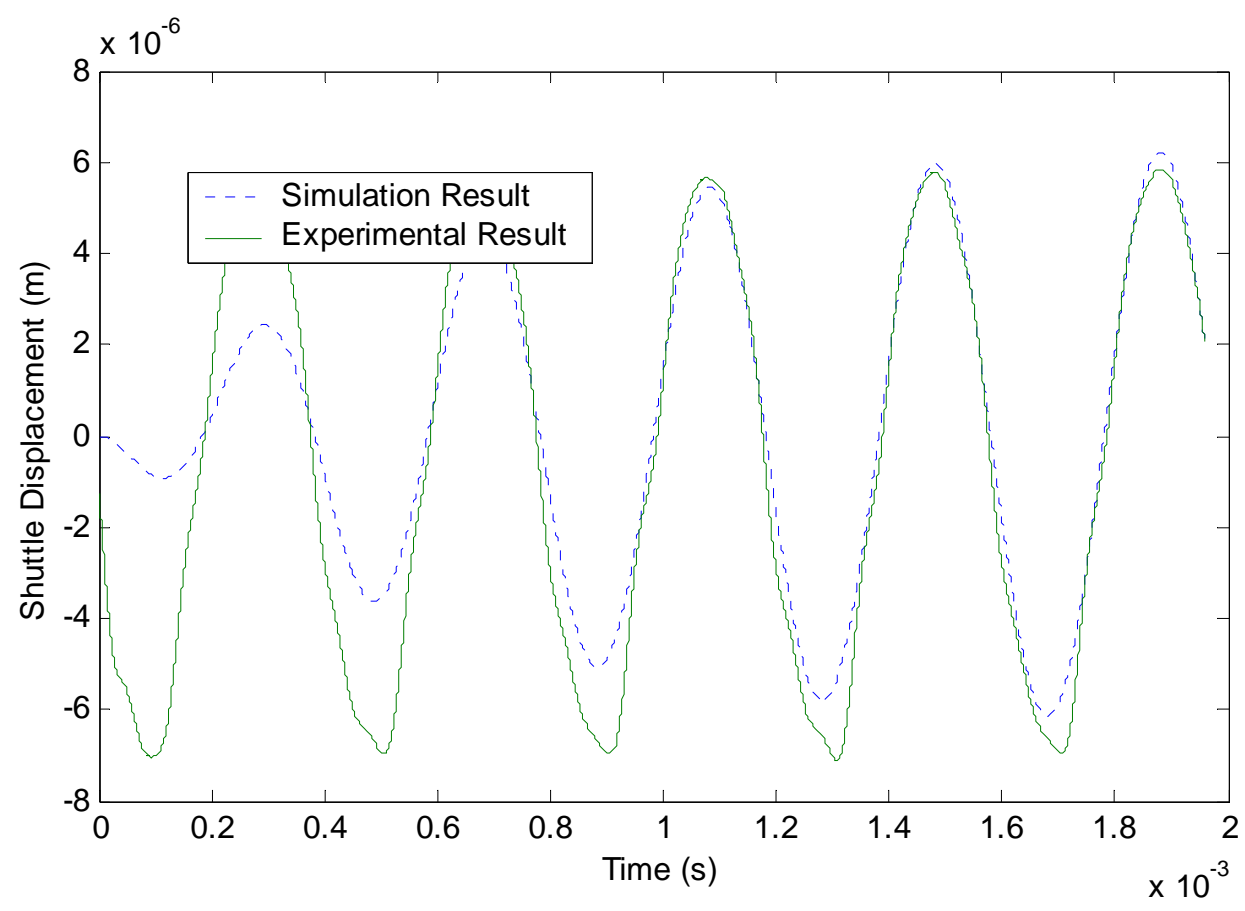

Figure 3-21 Displacement of LCR shuttle at $2500 \mathrm{~Hz}$ 


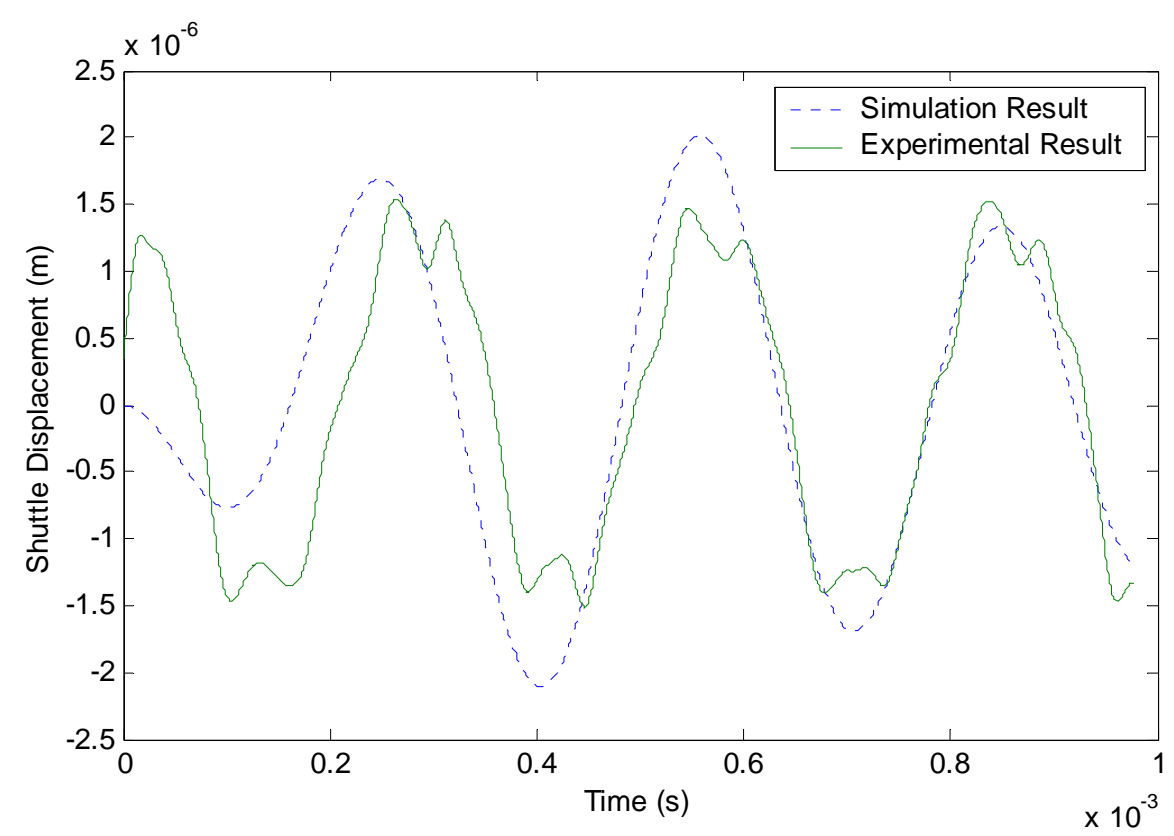

Figure 3-22 Displacement of LCR shuttle at $3500 \mathrm{~Hz}$

\subsubsection{Experimental system identification on grating structure device}

Two methods, frequency sweeping method and frequency domain method, have been applied to the grating structure device, and the results have been compared.

\subsubsection{Experimental implementation of frequency sweeping method}

Since the amplitudes and phases of the shuttle displacements are mandatory conditions for this parameter identification method, they must be revealed from the optical signal before system identification can be performed.

\section{Revealing amplitude and phase of position signal from optical signal}

An off-line method had been developed just based on the fact that for the optical signal, during the time interval from one peak/valley to its adjacent valley/peak, the shuttle travels $2 \mu \mathrm{m}$. The LCR is a linear $2^{\text {nd }}$ order system, which means, when the input to the LCR is purely sinusoidal, the position signal is also purely sinusoidal. If the force applied to the comb resonator is

$$
\text { Equation 3-26 } \quad F_{e}(t)=B \sin (\omega t+\beta) \text {, }
$$

the shuttle displacement (position signal) of the comb resonator can be described as 


$$
\text { Equation 3-27 } x(t)=A \sin (\omega t+\alpha),
$$

and the phase shift $\phi$ between this two signals is

$$
\text { Equation 3-28 } \phi=\alpha-\beta \text {. }
$$

Since $0<\phi<\pi$, it can be found that

$$
\text { Equation 3-29 } \beta<\alpha<\beta+\pi \text {. }
$$

If two particular points in time, $t_{1}$ and $t_{2}$, are chosen that satisfy

$$
\text { Equation 3-30 } x\left(t_{1}\right)+x\left(t_{2}\right)=0,
$$

the following equations will be true:

$$
\begin{gathered}
A \sin \left(\omega t_{1}+\alpha\right)+A \sin \left(\omega t_{2}+\alpha\right)=0, \\
\sin \left(\frac{\omega\left(t_{1}+t_{2}\right)+2 \alpha}{2}\right)=0, \\
\omega\left(t_{1}+t_{2}\right)+2 \alpha=2 k \pi
\end{gathered}
$$

then we can get

Equation 3-31 $\alpha=k \pi-\frac{\omega\left(t_{1}+t_{2}\right)}{2}=k \pi-f\left(t_{1}+t_{2}\right) \pi$.

From Equation 3-27, we also can get that

Equation 3-32 $A=\frac{x\left(t_{1}\right)}{\sin \left(\omega t_{1}+\alpha\right)}$.

With Equation 3-31 and Equation 3-32, the amplitudes and phases of the shuttle displacement can be found.

For example, by determining the local maximum/minimum of the optical signal shown in Figure 3-23, we can find the corresponding time for peaks 1 through 14 (listed in Table 3-8).

Table 3-8: Corresponding times for the occurrence of each peak.

\begin{tabular}{|c|c|c|c|c|c|c|c|}
\hline Peak & $\mathbf{P}_{\mathbf{1}}$ & $\mathbf{P}_{\mathbf{2}}$ & $\mathbf{P}_{\mathbf{3}}$ & $\mathbf{P}_{\mathbf{4}}$ & $\mathbf{P}_{\mathbf{5}}$ & $\mathbf{P}_{\mathbf{6}}$ & $\mathbf{P}_{\mathbf{7}}$ \\
\hline Corresponding Time (ms) & 0.516 & 1.272 & 2.136 & 3.032 & 3.838 & 4.606 & 5.472 \\
\hline Peak & $\mathbf{P}_{\mathbf{8}}$ & $\mathbf{P}_{\mathbf{9}}$ & $\mathbf{P}_{\mathbf{1 0}}$ & $\mathbf{P}_{\mathbf{1 1}}$ & $\mathbf{P}_{\mathbf{1 2}}$ & $\mathbf{P}_{\mathbf{1 3}}$ & $\mathbf{P}_{\mathbf{1 4}}$ \\
\hline Corresponding Time (ms) & 6.362 & 7.172 & 7.944 & 8.810 & 9.696 & 1.050 & 11.274 \\
\hline
\end{tabular}


For this signal, the choice of $t_{p 2}$ as $t_{1}$ and $t_{p 3}$ as $t_{2}$ results in the phase of the position signal being expressed as:

$$
\alpha=k \pi-300(1.272 e-3+2.136 e-3) \pi=(k-1.0224) \pi=3.0712(k=2),
$$

and the amplitude of position signal can be expressed as

$$
A=\frac{x\left(t_{1}\right)}{\sin \left(\omega t_{1}+\alpha\right)}=\frac{2 e-6}{\sin (2 \pi \times 300 \times 1.272 e-3+(k-1.0224) \pi)}=2.7501 e-6(\mathrm{~m}) .
$$

Similarly, by choosing different $t_{1}$ and $t_{2}$, a group of amplitude and phase values can be found, as listed in Table 3-9.

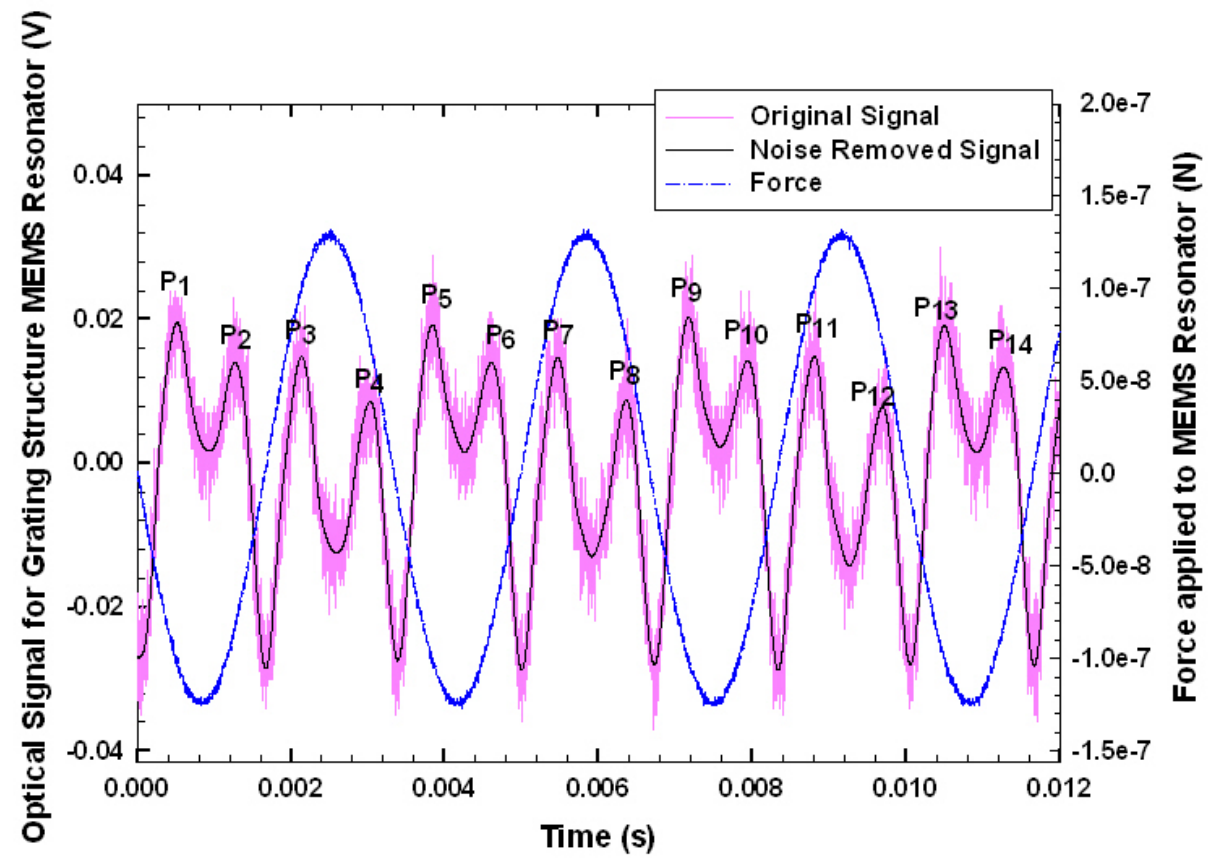

Figure 3-23: Force and its Optical Output for a MEMS resonator (the force frequency is $300 \mathrm{~Hz}$ )

Table 3-9: Phase and amplitude for various time values.

\begin{tabular}{|l|c|c|c|c|c|c|}
\hline & $t_{1}=t_{P 2}$ & $t_{1}=t_{P 4}$ & $t_{1}=t_{P 6}$ & $t_{1}=t_{P 8}$ & $t_{1}=t_{P 10}$ & $t_{1}=t_{P 12}$ \\
& $t_{2}=t_{P 3}$ & $t_{2}=t_{P 5}$ & $t_{2}=t_{P 7}$ & $t_{2}=t_{P 9}$ & $t_{2}=t_{P 11}$ & $t_{2}=t_{P 13}$ \\
\hline$\alpha$ (rad) & 3.0712 & 2.9500 & 3.0681 & 2.9595 & 3.0593 & 2.9550 \\
\hline $\mathbf{A ~ ( n m ) ~}$ & 2.7501 & 2.9042 & 2.7452 & 2.8927 & 2.7452 & 2.9042 \\
\hline
\end{tabular}


By eliminating the highest and lowest values and averaging the remaining values, the amplitude and phase that will be used in the parameter identification can be obtained. Based on this consideration, the amplitude for this particular optical signal is 2.8236, while the phase is $3.0093 \mathrm{rad}$. Then the gain and phase delay can be calculated with the same method described in section 3.3.1.2.

The gains and phase delays for other frequencies were calculated in the same way, and with the frequency sweeping method mentioned in chapter 2, the system parameters can be identified as

$$
\begin{aligned}
m & =2.9595 \times 10^{-10}, \\
\beta & =1.0080 \times 10^{-6}, \\
k_{s} & =0.030028 .
\end{aligned}
$$

Figure 3-24 shows the curve fitting result for this LCR system. This set of parameters will be used to perform feedback control on the MEMS lateral comb resonator later. 

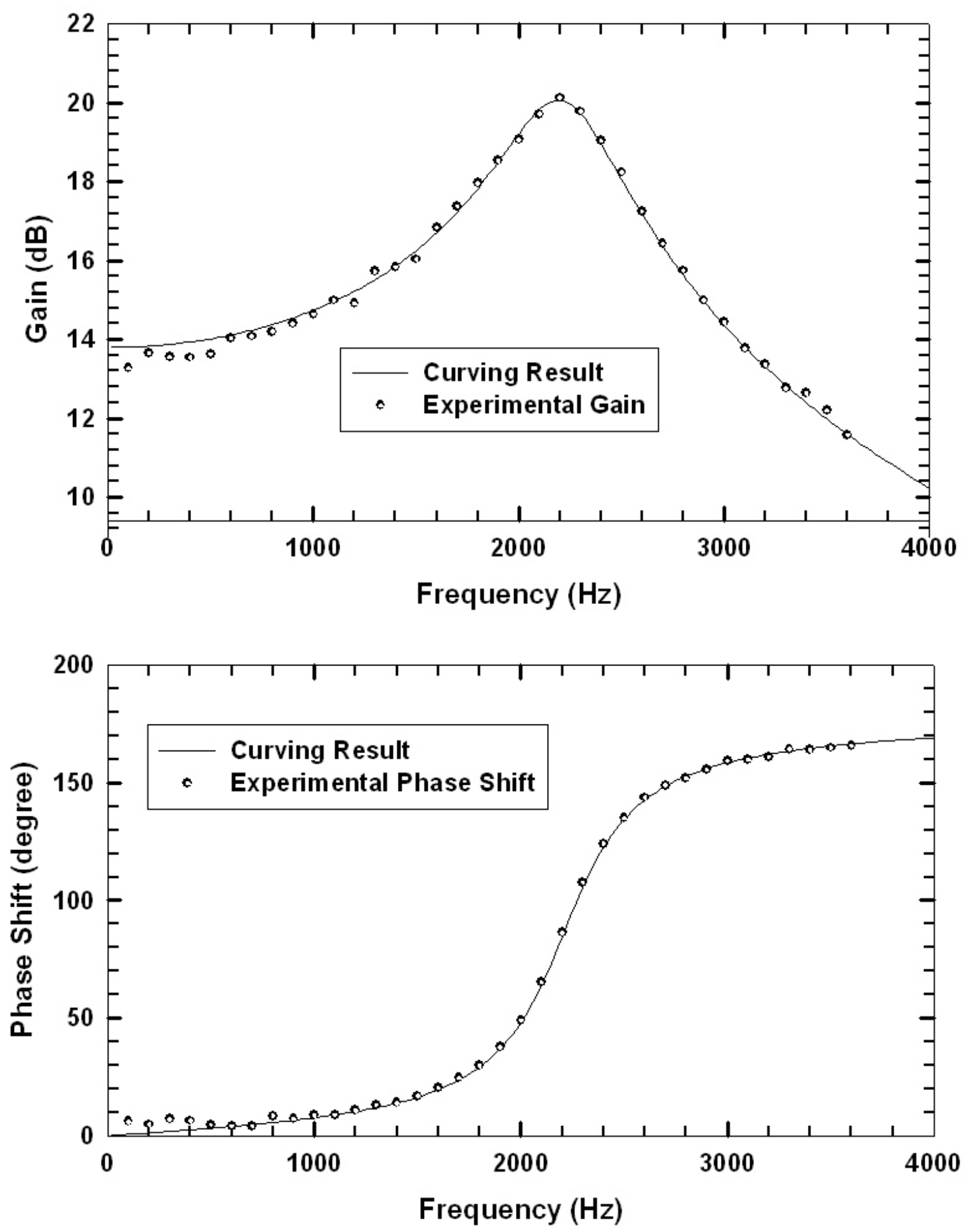

Figure 3-24: Curve fitting result for LCR with beam length $400 \mu \mathrm{m}$ 


\subsubsection{Experimental implementation of frequency domain method}

The frequency domain parameter identification method that introduced in 3.2.2 has been experimentally implemented again with the grating structure LCR.

The same device that was used in the frequency sweeping system identification experiment has been utilized in this experimental implementation. In this experiment, \pm 10 volts DC was applied to the stators, while AC voltage Vs that was generated by dSpace 1103 controller was applied to the stage of this LCR, where

$$
V s=3.4 \sin (2 \pi \cdot 100 t)+2.4 \sin (2 \pi \cdot 150 t)+3 \sin (2 \pi \cdot 330 t) \mathrm{V} .
$$

The normalized power spectrum of this LCR's output and the genetic algorithm curve fitting results are plotted in Figure 3-25.

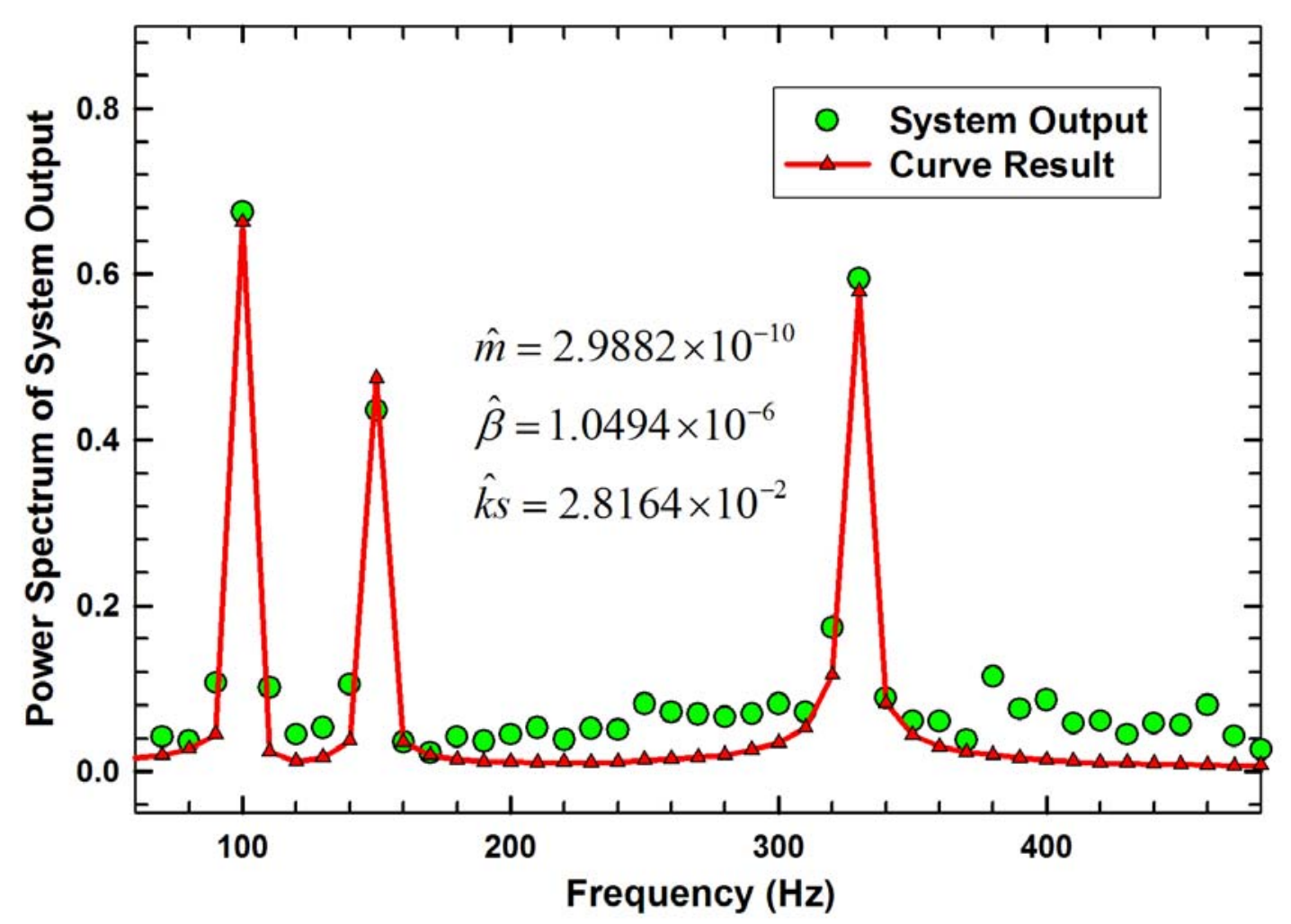

Figure 3-25: Parameter Estimation Result for the MEMS LCR 
Table 3-10: Parameter estimation results

\begin{tabular}{|c|c|c|c|}
\hline & Mass (m) & Spring Constant $\left(\boldsymbol{k}_{\boldsymbol{s}}\right)$ & Damping $(\boldsymbol{\beta})$ \\
\hline Frequency Sweep & $2.9595 \times 10^{-10}$ & $3.0028 \times 10^{-12}$ & $1.0080 \times 10^{-6}$ \\
\hline Frequency Domain system ID & $2.9882 \times 10^{-10}$ & $2.8164 \times 10^{-12}$ & $1.0494 \times 10^{-6}$ \\
\hline
\end{tabular}

The estimation results obtained from both methods are very close, which means the estimated parameters are very close to what they are. Based on previous analysis results that the frequency sweeping method has better performance, the parameters estimated with that method would be used in later closed-loop control efforts.

\subsubsection{Experimental system identification on a non-linear device}

Slight modification has been made on the frequency sweeping method to perform the parameter identification on a non-linear MEMS device, the Parallel Plates Resonator (PPL). Like LCR, the PPL resonator was designed at WVU and fabricated by Chronos Integrated Microsystems' Multi-User MEMS Process service (MUMPs). As shown in Figure 3-26, the bottom plate of this PPL resonator is fixed, while the upper plate is held by four slim legs. When there is voltage difference between the plates, vertical motion will be observed on the upper plate.

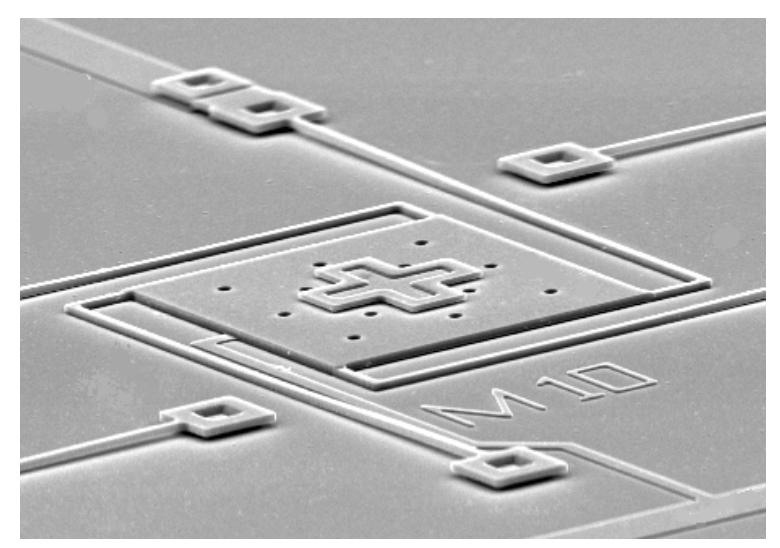

Figure 3-26: Micro Parallel Plate Resonator

The mathematical model of this PPL system can also be described as

Equation 3-33

$$
F_{e}=m \ddot{x}+\beta \dot{x}+2 k_{s} x+F_{d},
$$

where $x$ denotes the z-direction displacement of the upper plate. The force between two plates is given by [73] 
Equation 3-34 $\quad F e=\frac{\varepsilon A}{2 x^{2}} V^{2}$,

which makes the system a non-linear system. Experimentally, the laser doppler vibrometer has been used to collect z-direction motion data for this PPL resonator.

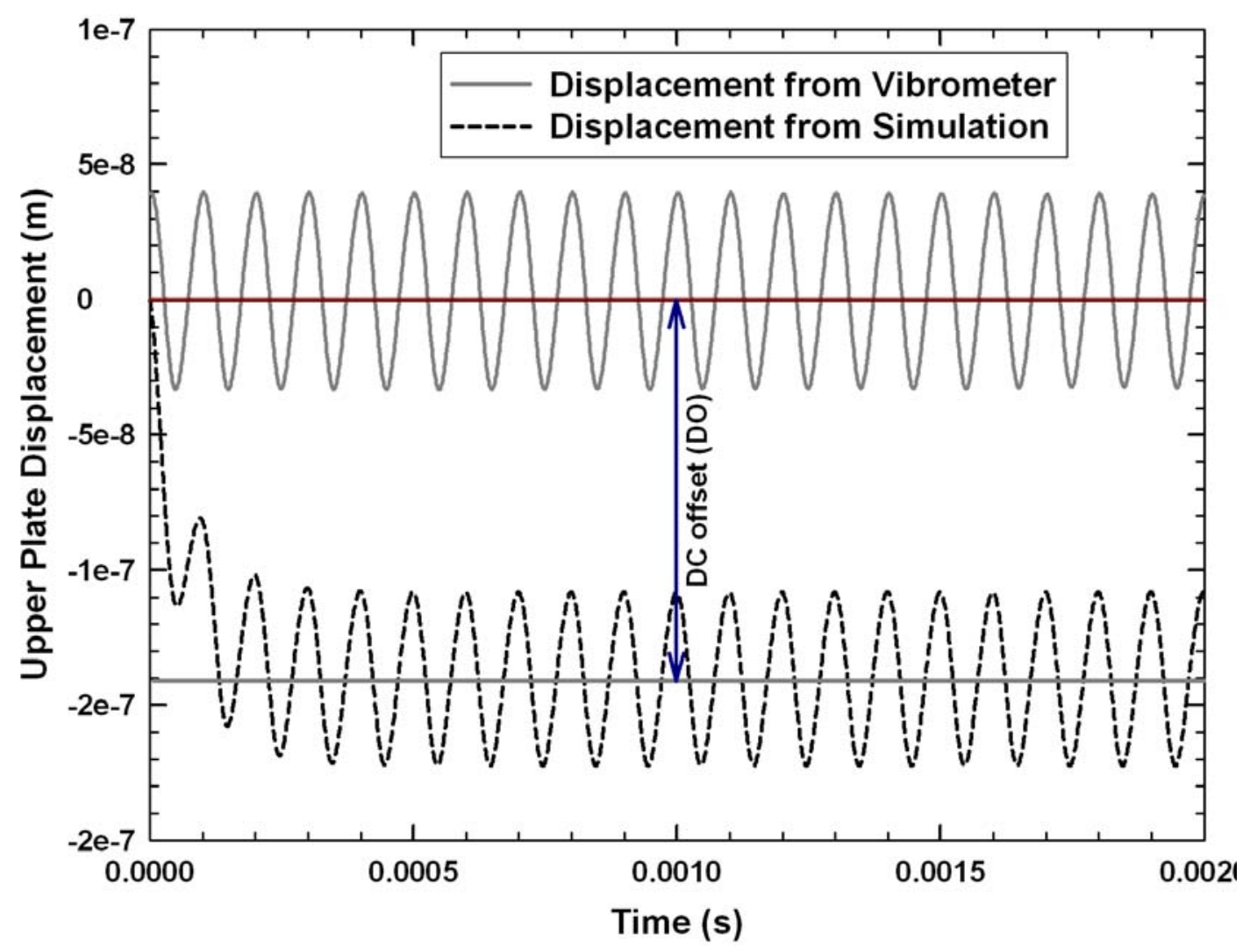

Figure 3-27: $\quad$ Upper plate's displacement of PPL--- force frequency $10 \mathrm{k} \mathrm{Hz}$

To perform the parameter identification, a $-4 \mathrm{~V}$ DC voltage has been applied to the bottom plate of a PPL, while 4V peak-peak AC voltage has been applied to its upper plate. As a result, the upper plate vibrated around a certain point at the same frequency as that of the AC voltage applied to it. Theoretically, three criteria can be used to identify the system parameters, they are 1) the DC offset (DO) as shown in Figure 3-27), 2) the phase difference between input and output, and 3) the 'gain', the ratio between the amplitude of steady state output and the amplitude of input. However, since we only have a velocity module for the vibrometer, even though the relative position of the upper plate can be obtained by integrating the velocity of the upper plate, the DC offset will be lost 
during the process (Figure 3-27, displacement from vibrometer). Therefore, only the last two criteria will be used in the parameter identification process.
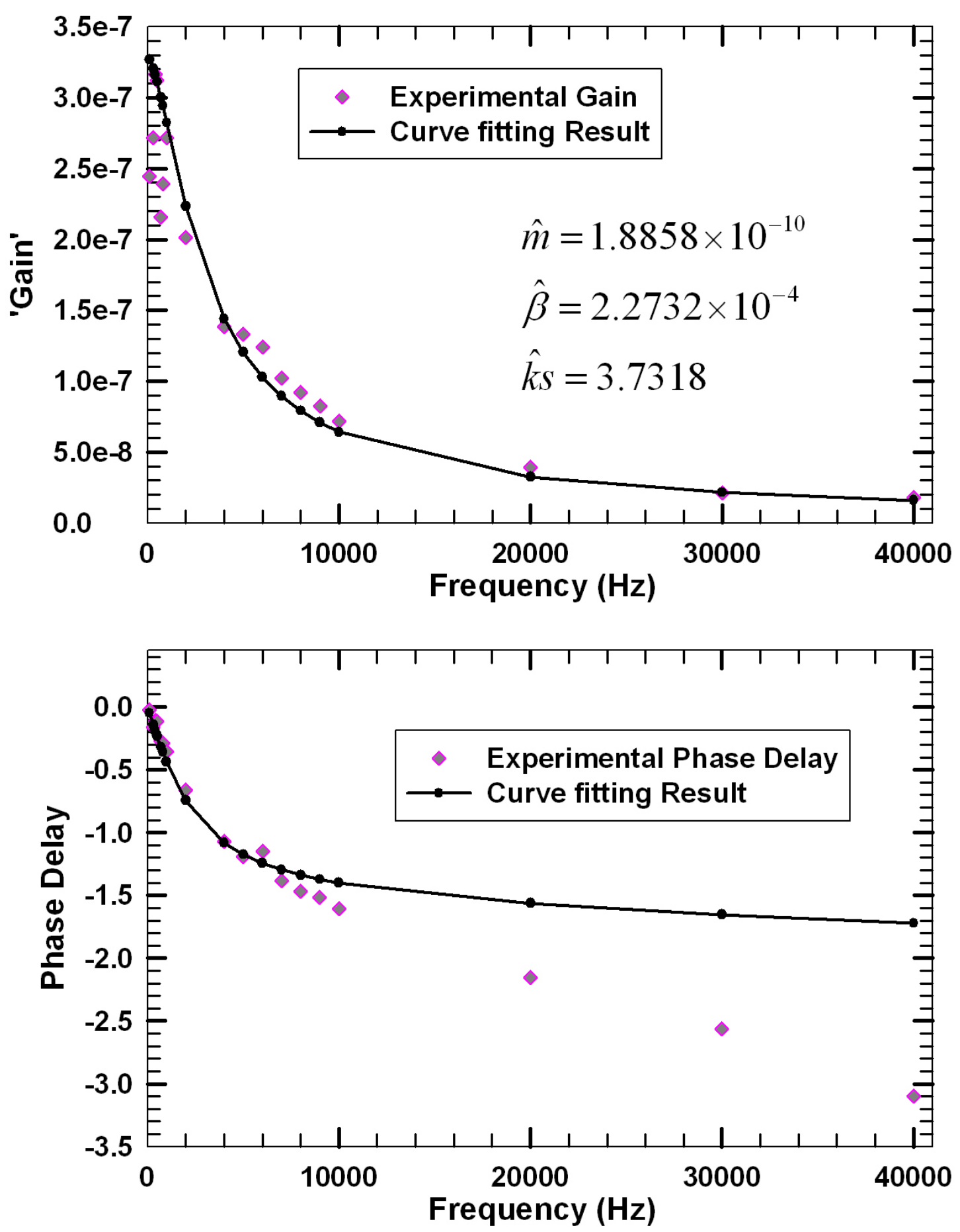

Figure 3-28: Curve fitting Result for Parallel Plate, 'gain'—amplitude of displacement divided by the amplitude of input AC voltage 
Again, the Genetic Algorithm method has been used to curve fit the experimental 'Gain' and phase shift. Unlike the 'gain' curve, when the frequency is beyond $10 \mathrm{k} \mathrm{Hz}$, a good match cannot be achieved on the phase delay curve. Research shows that the mismatch of the phase delay curve after $10 \mathrm{k} \mathrm{Hz}$ is caused by the compressible squeeze film behavior [74]. Therefore, the phase delay after $10 \mathrm{k} \mathrm{Hz}$ won't be considered during this system identification. And the parameters of this PPL have been estimated as

$$
\begin{aligned}
& \hat{m}=1.8858 \times 10^{-10}, \\
& \hat{\beta}=2.2732 \times 10^{-4}, \text { and } \\
& \hat{k}_{s}=3.7318 .
\end{aligned}
$$

With theoretical calculation that based on geometry and mass density of the polysilicon, the mass of the upper plate is $1.8142 \times 10^{-10}$, which is very close to the estimated results. 


\section{Chapter 4 Feedback Control on Single Opening MEMS Lateral Comb Resonator}

The lateral electrostatic comb drive resonator (LCR) fabricated by Chronos Integrated Microsystems' Multi-User MEMS Process service (MUMPs) presents linear oscillatory motion in the lateral direction. The basic resonator could provide a continuous oscillatory motion to a gear or other micro mechanism. Therefore, the implementation of feedback control on this kind of device could not only widen its applications, but also improve its reliability dramatically.

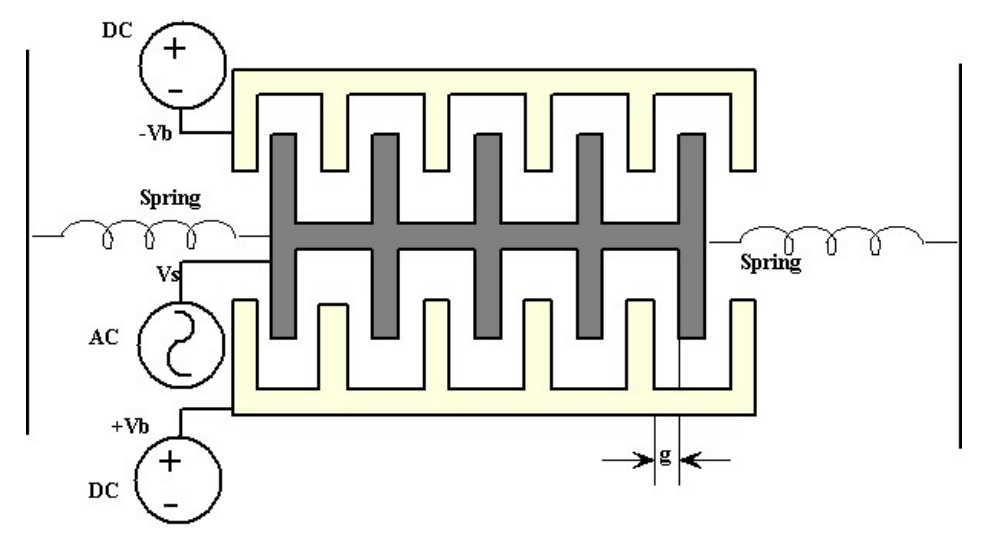

Figure 4-1: Schematic drawing of comb-drive actuator.

\subsection{System Analysis}

For the MEMS LCR system (Equation 3-1), when substituting $x$ with $x_{1}$, and $\dot{x}$ with $x_{2}$, system can be rewritten as

Equation 4-1

$$
\left\{\begin{array}{l}
\dot{x}_{1}=x_{2} \\
\dot{x}_{2}=-\frac{2 k_{s}}{m} x_{1}-\frac{\beta}{m} x_{2}+\frac{1}{m} F_{e}-\frac{1}{m} F_{d} . \\
y=x_{1}
\end{array}\right.
$$

With zero load $\left(F_{d}=0\right)$, the LCR can be described as

$$
\begin{aligned}
& \dot{X}=A X+B u \\
& Y=C X
\end{aligned}
$$


where

$$
X=\left[\begin{array}{l}
x_{1} \\
X_{2}
\end{array}\right], u=F_{d}, A=\left[\begin{array}{cc}
0 & 1 \\
-\frac{2 k_{s}}{m} & -\frac{\beta}{m}
\end{array}\right], B=\left[\begin{array}{c}
0 \\
\frac{1}{m}
\end{array}\right] \text {, and } C=\left[\begin{array}{ll}
1 & 0
\end{array}\right] \text {. }
$$

The controllability matrix of this system is

$$
P=\left[\begin{array}{ll}
B & A B
\end{array}\right]=\left[\begin{array}{cc}
0 & \frac{1}{m} \\
\frac{1}{m} & -\frac{\beta}{m^{2}}
\end{array}\right] .
$$

Since both $m$ and $\beta$ greater than zero, the rank of the controllability matrix is 2 , that means the LCR system is completely controllable.

For the linear LCR, the movement of its shuttle is determined by the electrostatic force that is generated by the overlapped fingers, while the electrostatic force is determined by the product of AC and DC voltages that are applied to the LCR. As shown in Equation 3-2, when applying DC voltages with equal value and opposite sign to the stators, the relationship between the electrostatic force and the voltages' product is linear.

In this study, the control of the LCR's shuttle motion was implemented by adjusting the voltage(s) that are applied to the LCR with different designed controllers. As previously mentioned, for the single opening device, the optical observing signal is proportional to the shuttle's displacement, therefore, we defined that

Equation 4-2

$$
O_{s}=R \cdot x \text { or } x=r \cdot O_{s},
$$

where $O_{s}$ is the optical observing signal, $x$ is the shuttle displacement, while $R \& r$ are the constant rate between $O_{s}$ and $x$.

\subsection{Active Control of Damping Shock Effects}

An external sudden strike can cause a sudden unwanted movement of the lateral comb resonator (LCR). This can be alleviated by implementation of a closed-loop control to minimize the motion. An experiment has been performed to damp the shock effect by means of closed-loop control. From Equation 3-2, we know that the electrostatic forces that are generated by the comb fingers are determined by both $\mathrm{V}_{\mathrm{s}}$ that is applied to the shuttle and $\mathrm{V}_{\mathrm{b}}$ that applied to the stators. In this experiment, the voltages were applied to the stators have been used as the control signal, while an impulse voltage forced the shuttle of the LCR to simulate the sudden strike. Under open-loop condition, as long as 
the amplitude of the impulse is within a safe range, the shuttle will oscillate at its resonance frequency and is not ideal for the LCR's application. Besides, if the amplitude of the impulse exceeds the safe range, the shuttle will hit the stator and cause permanent damage to the device.

Both open loop and closed-loop experiments have been implemented and the results have been compared. In the open loop operation, the stators are forced with positive and negative 8 volt DC respectively, while a continuous pulse with 10 volt in magnitude and $10 \mu$ s in width generated by a signal generator has been applied to the shuttle (Figure 4-3). The behavior of the shuttle was observed with the through wafer optical monitoring technique. The impulse that applied on the shuttle introduced a $2.5 \mathrm{volt}$ sudden change on the optical intensity (Figure 4-4).

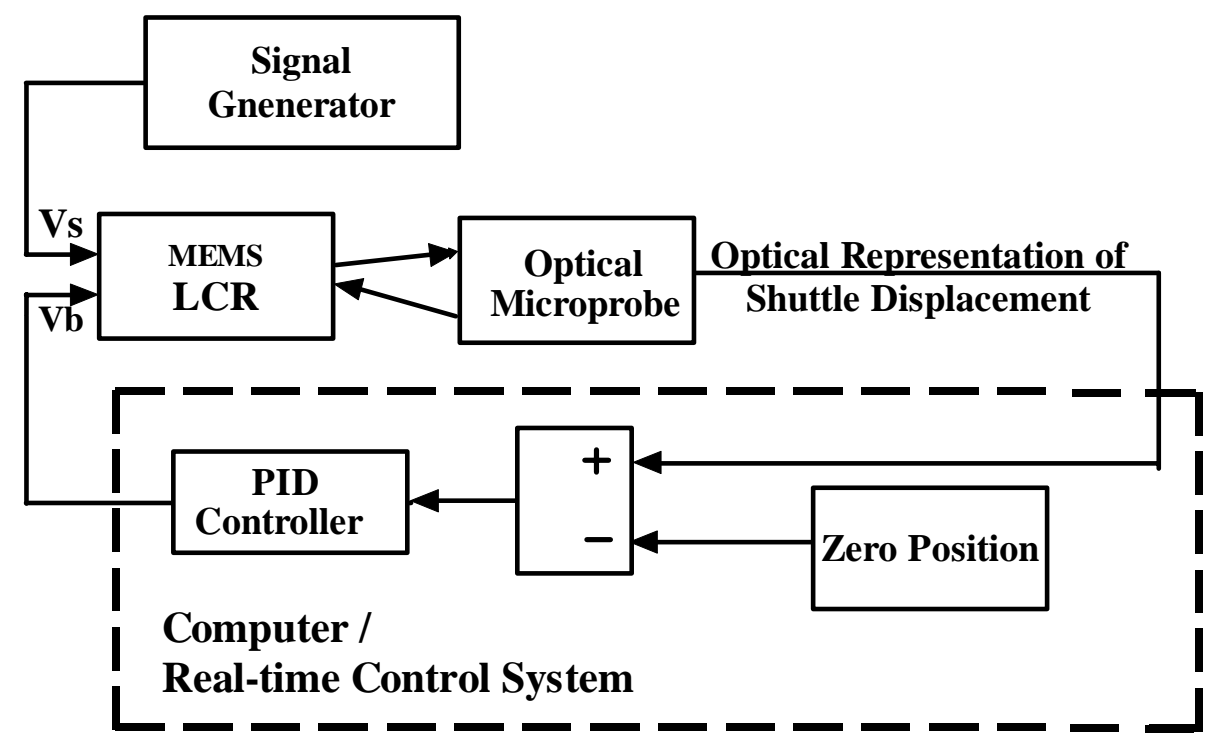

Figure 4-2: Block Diagram of the shock Damping System

This unwanted effect can be damped by means of closed-loop control with the following PID controller

$$
\text { Equation 4-3 } \quad V_{b}=K_{p} \cdot \varepsilon+K_{i} \cdot \int \varepsilon+K_{d} \cdot \dot{\varepsilon} .
$$

where $\varepsilon=R \cdot x-O_{s 0}$, and the rate $R$ is estimated based on the open-loop result and its corresponding simulation result. The proportional gain, $K_{p}$, was initially designed by 
Linear-Quadratic Regulator and then divided by R, while both $K_{i}$ and $K_{d}$ were set to zero as the initial values and were manually adjusted during the experiment.

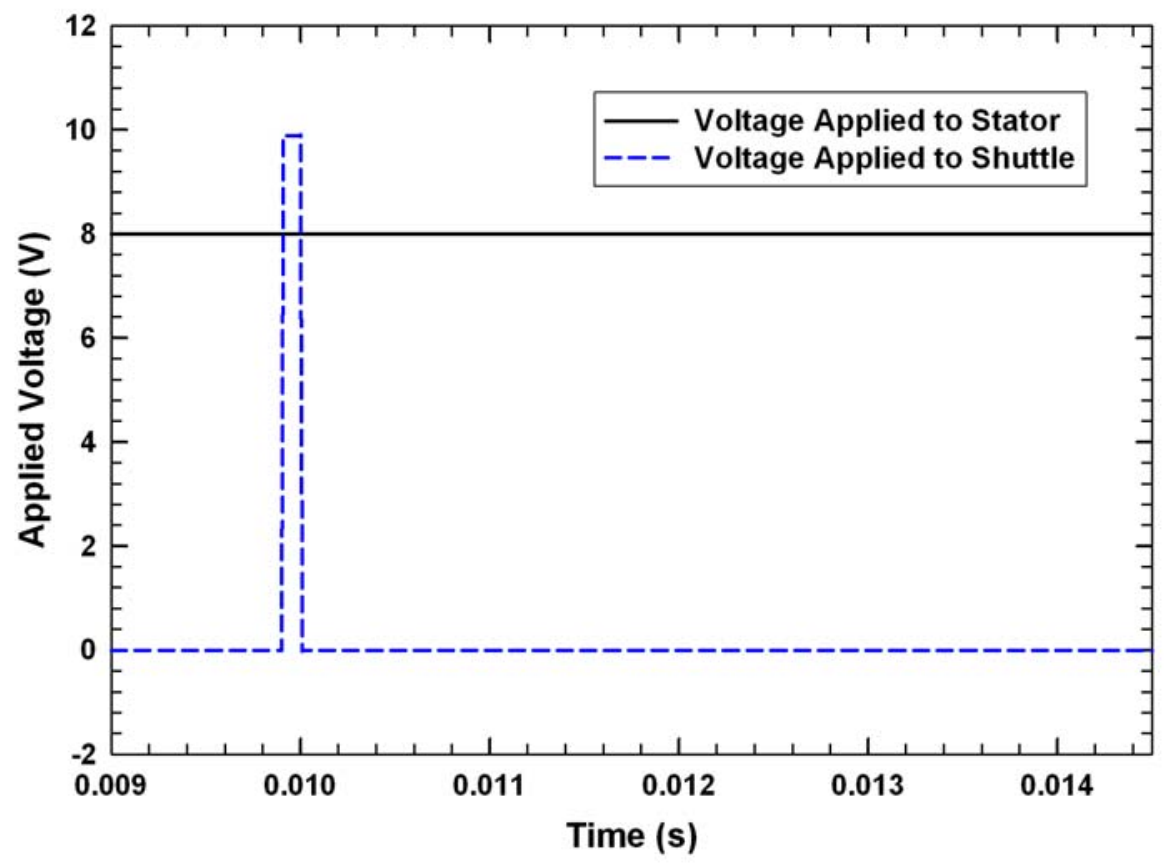

Figure 4-3: Voltages applied to the LCR during the open-loop experiment

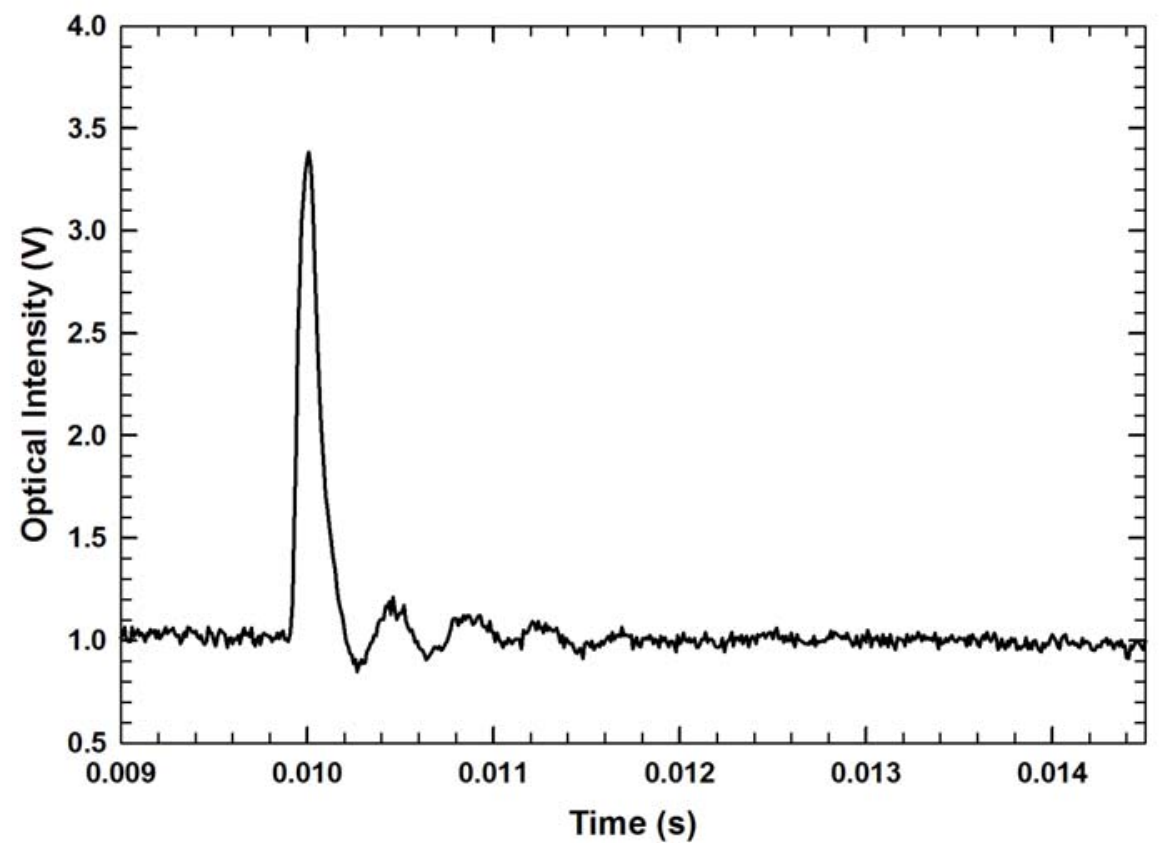

Figure 4-4: Open-loop result when an impulse within safety range was applied to the shuttle 


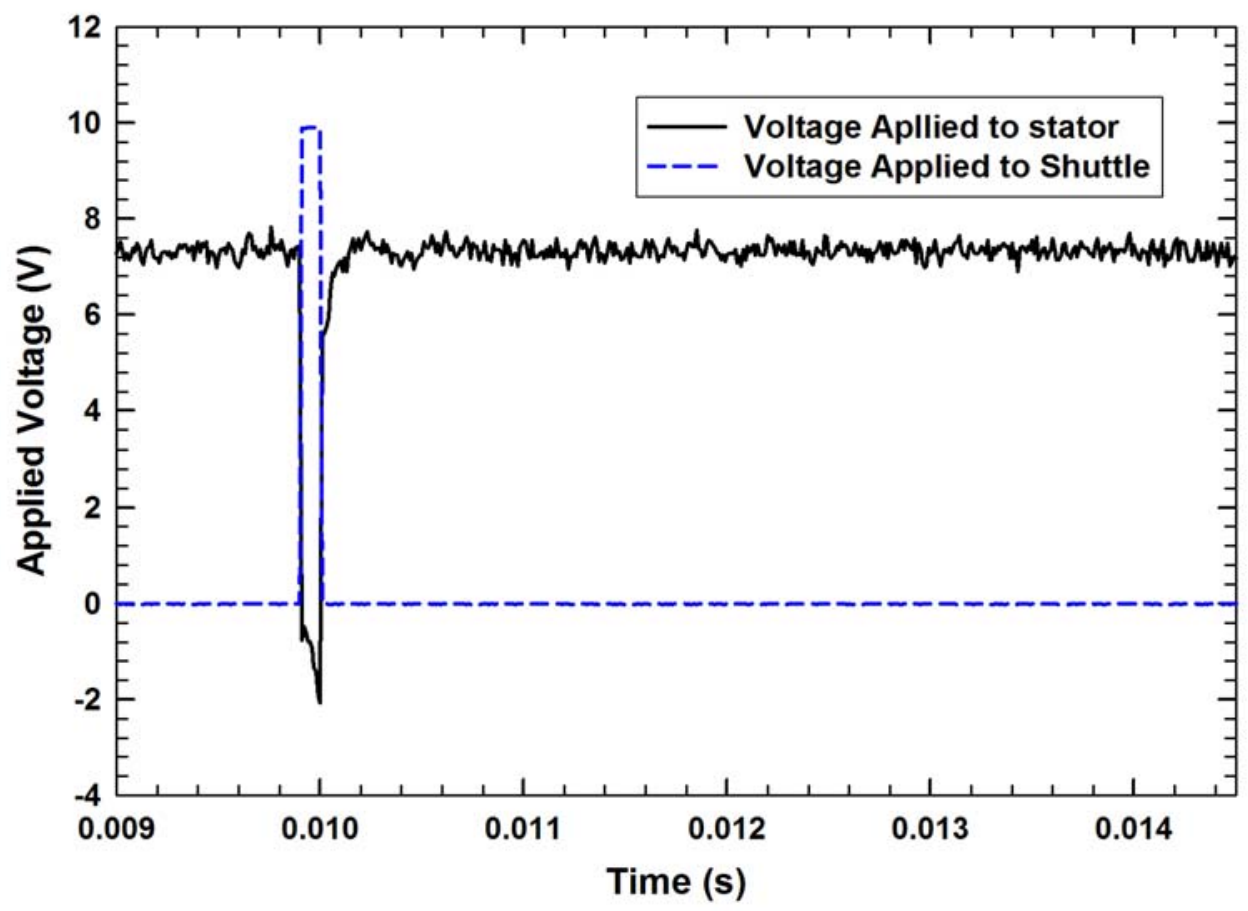

Figure 4-5: Voltages applied to the LCR during the closed-loop experiment

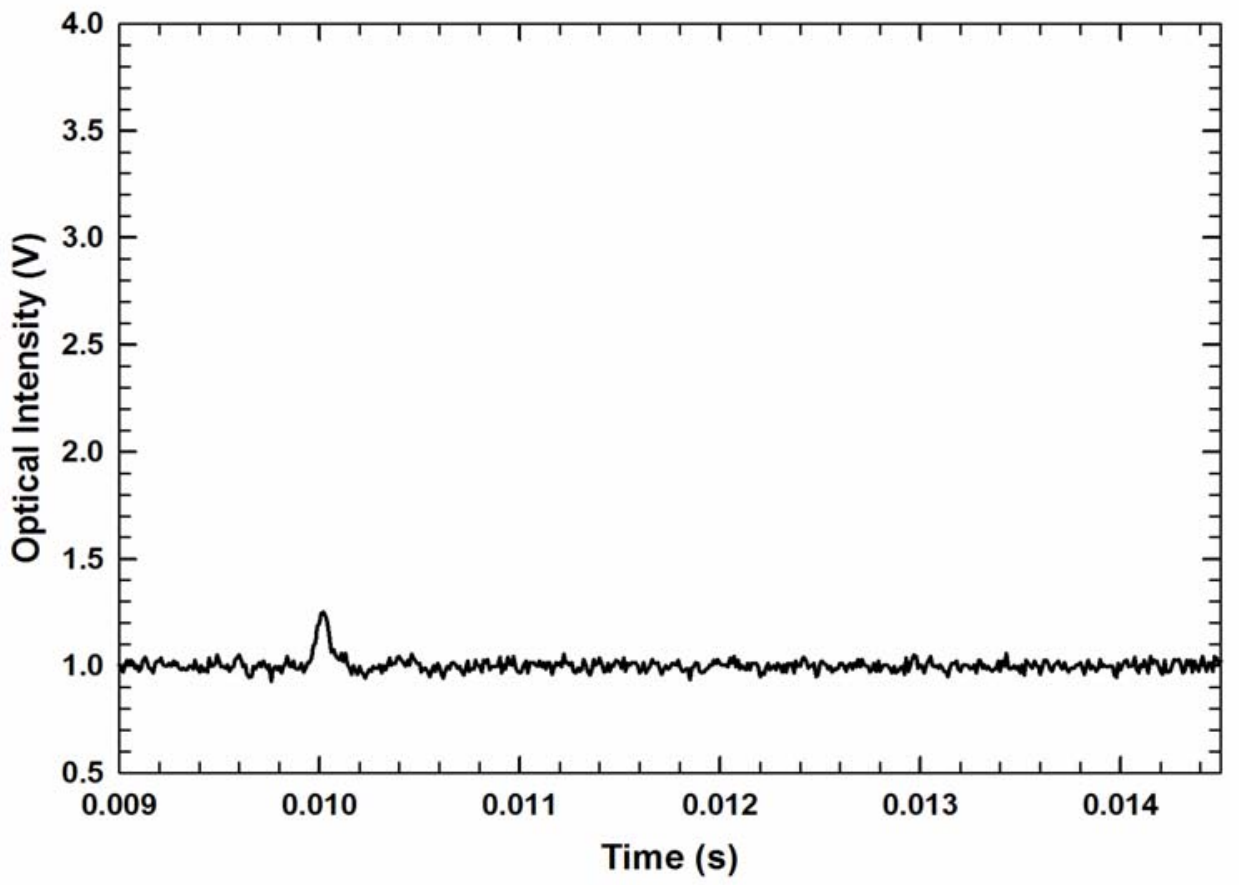

Figure 4-6: Closed-loop result when an impulse within safety range was applied 
As shown in Figure 4-2, the through wafer optical monitoring technique has been used to monitor the feedback signal (the optical representative of the shuttle's displacement), and the real time controller used in this system was implemented by a dSPACE's DS1103 PPC Controller Board. The gains of the PID controller can be adjusted manually during the experiment to achieve better control result.

In the closed-loop implementation, same impulse as used in the previous openloop experiment has been applied to the shuttle, while the output voltage of the controller $V b s$ was applied to the stators. In this experiment, the strike that is caused by the impulse has been damped to less than 0.4 volt (Figure 4-6). Compared with the open-loop result, more than $80 \%$ of the strike has been damped by the implementation of this closed-loop control.

\subsection{Stroke-length Control}

Because of the inherent low inertia of the LCR, the stroke length of the shuttle can vary significantly due to disturbance and/or loading conditions. Depending upon the applications, especially those involving mechanical power transfer to other micro-sized devices, the stroke-length may vary with respect to the amount of power transferred. The uncertainty of the stroke-length will become even more profound under load conditions.

The successful performance of closed-loop control implemented that was reported in [2] to restore the shuttle's stroke-length to its original value needs to be improved. The response time of restoring the displacement was about one second, and success was achieved only when driven under low frequency $(<=100 \mathrm{~Hz})$ condition. Since the lateral electrostatic comb drive resonator is designed to be operated at higher frequency, a real time controller with short response time is necessary to obtain a quick response. An improved real-time PID controller was evaluated in this study.

Before the closed-loop implementation, an open-loop experiment was performed. In the open-loop experiment, a constant 7V DC was applied to the LCR's stator, while a $1000 \mathrm{~Hz}$ AC voltage was applied to the shuttle. The initial amplitude of the AC voltage is $6 \mathrm{~V}$, then increased to $9 \mathrm{~V}$ after around $17 \mathrm{~ms}$, and as shown in Figure 4-7, the optical representative of the shuttle displacement also increased from $0.5 \mathrm{~V}$ to $0.75 \mathrm{~V}$ in the same time. 

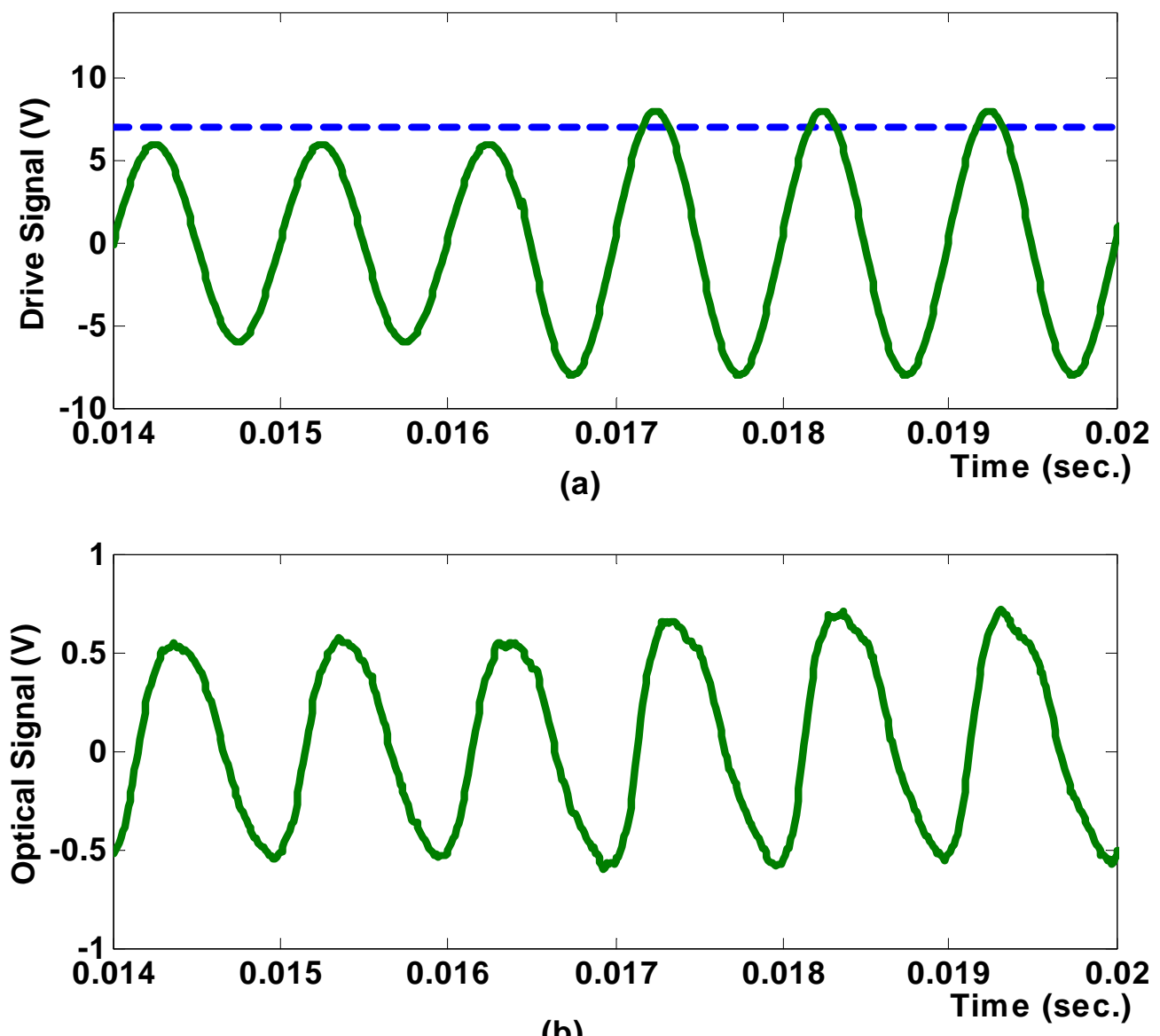

(b)

Figure 4-7 Open loop experiment result for comb drive actuator

The PID controller is similar to the one that was used in the previous shock damping control, and it can be described as

Equation 4-4

$$
V_{b}=K_{p} \cdot \varepsilon+K_{i} \cdot \int \varepsilon+K_{d} \cdot \dot{\varepsilon} .
$$

This time, the error was defined as $\varepsilon=P(R \cdot x)-p_{d}$, where $P(R \cdot x)$ is the peak value of the optical signal, and $p_{d}$ is the desired optical peak value. The gains of this controller are determined in the same way as that in the previous shock damping control experiment. 
From the mathematical description of this LCR, it is easy to show that the frequency of the shuttle's movement is the same as the frequency of the AC voltage that was applied to the LCR. A peak detector has been developed based on this fact. A first-in first-out dynamic buffer has been used with the peak detector, the capacity of the buffer is determined by both A/D sample rate SR and the frequency of the AC voltage Freq, and the capacity $C=S R /$ Freq . The buffer was initialized with all zeros, and when the peak detector began to work, the optical signal was fed into the buffer, the maximum value in the buffer was outputted from the peak detector as the peak value of the optical signal. Both simulation and experiment provided good peak estimation results. However, device failure could lead to peak detector malfunction since the frequency of the shuttle displacement might differ from the AC input.

To avoid malfunction, a frequency detector has been built, implemented and used in this shuttle stroke length control experiment. The frequency of the shuttle displacement is estimated by counting the zero-crossings in the frequency detector. Each time when the difference between the estimated frequency and force frequency (AC frequency) exceeds $5 \%$ of the force frequency, a fault alarm will be sent out indicating there might be a device failure. If continuous alarms are detected, the PID controller will be shutdown automatically.

Figure 4-8 shows the block diagram of the closed-loop system. Frequency detector, peak detector, and PID controller are all implemented by the DSP1103 controller board. In the closed-loop implementation, a $1000 \mathrm{~Hz}$ AC voltage was applied to the shuttle, while the PID controller supplied the voltages that were applied to the stators. 


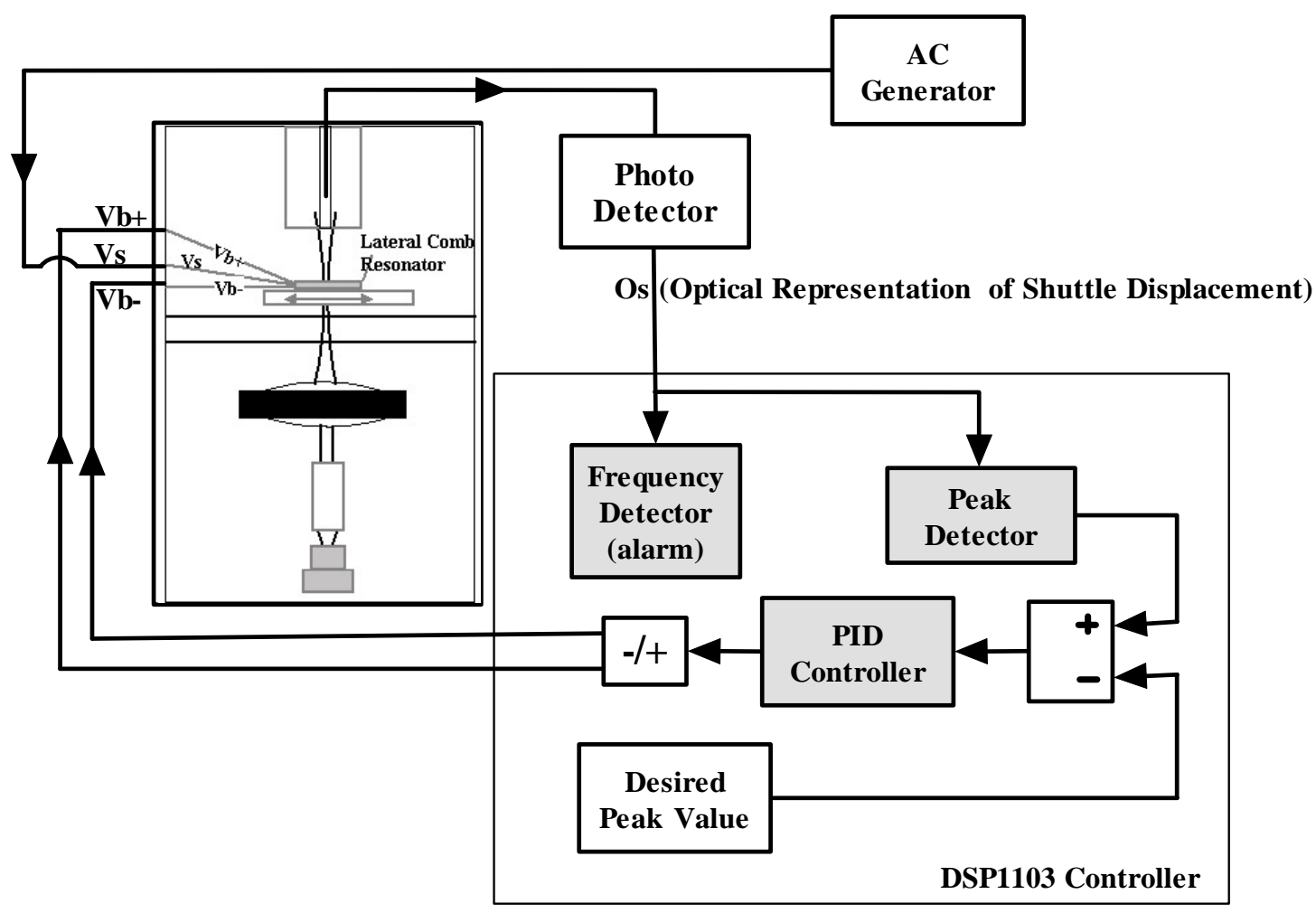

Figure 4-8 Block Diagram of the Closed-loop System

Similar to the open-loop implementation, the amplitude of the AC voltage is $6 \mathrm{~V}$ at the beginning, and increased to $9 \mathrm{~V}$ in $9 \mathrm{~ms}$, however, unlike the open-loop result, the shuttle stroke length was restored to $0.5 \mathrm{~V}$. The result has been plotted in Figure 4-9, the amplitude of stroke-length was restored within 1 cycle when operating at $1000 \mathrm{~Hz}$. 


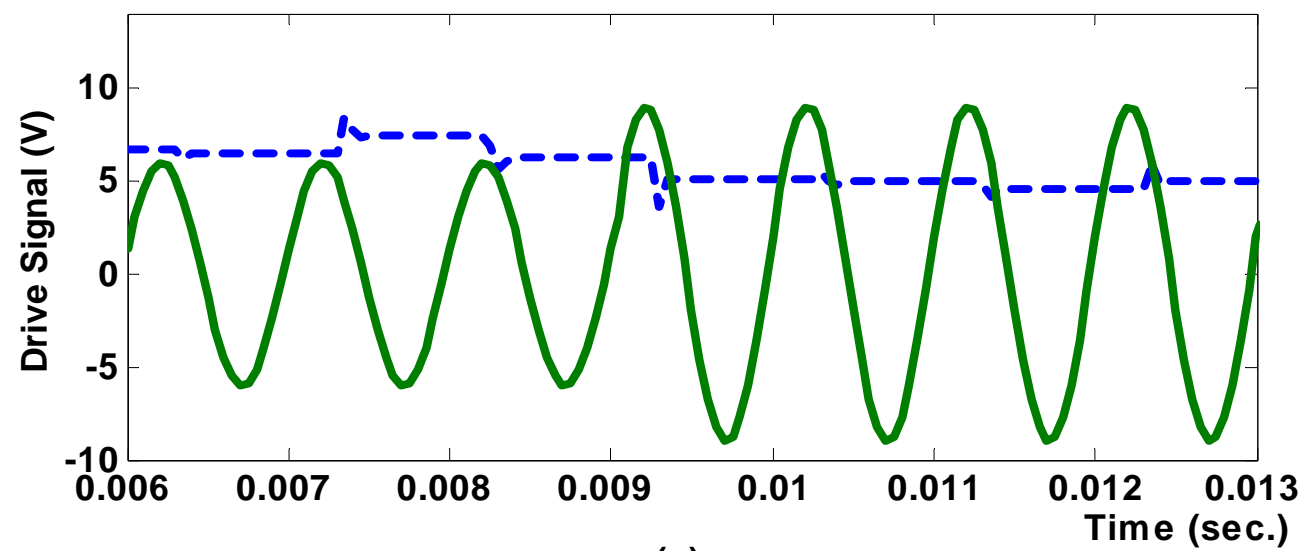

(a)

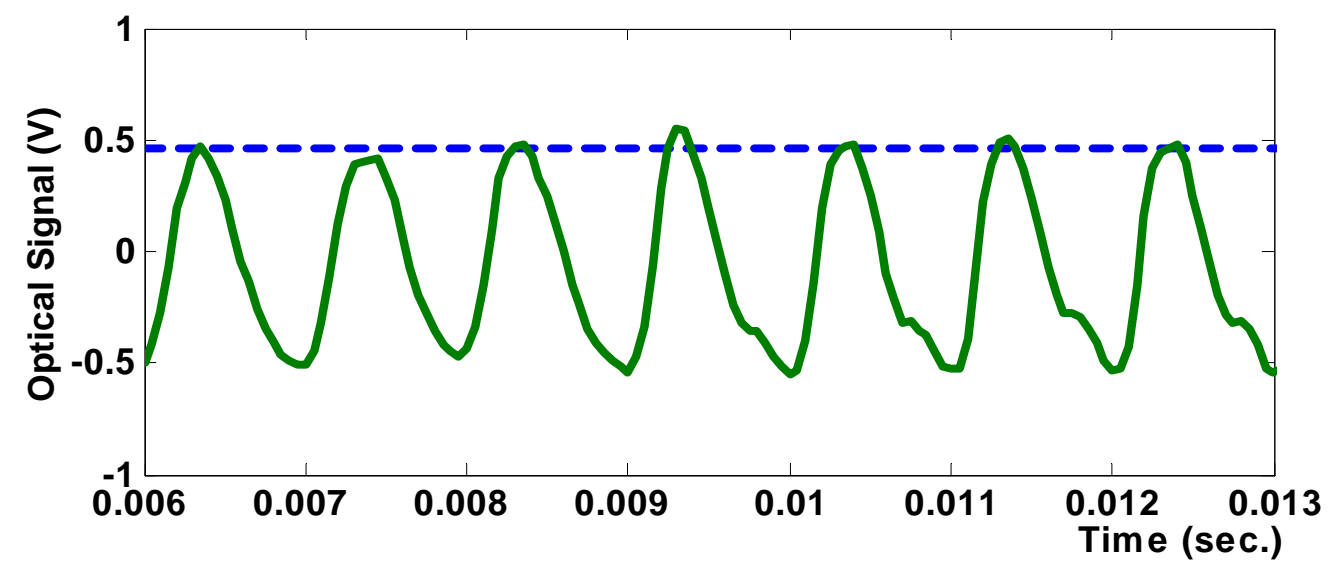

(b)

Figure 4-9 Closed-loop control experiment result for comb drive actuator 


\section{Chapter 5 Feedback Control on Grating Structure MEMS Lateral Comb Resonator}

Experimental success has been achieved for closed-loop control on single opening lateral comb resonators by using the output of a through-wafer optical microprobe as a feedback signal [75]. The unit of shuttle displacement shown in those experimental results is given in volts instead of meters due to the uncertainty of the ratio between the optical signal and position signal. It is difficult to determine the absolute displacement of the shuttle since the level of the output waveform is directly related to the coupled optical intensity. A comb resonator utilizing a grating structure has been designed in order to overcome this uncertainty [76]. For the grating structure device, the optical output is no longer proportional to the position signal, instead, the optical probe beam produces an encoder-like signal which provides a precise position signal with an absolute resolution of 2 micrometers.

Figure 5-1 shows the optical output of a grating structure comb resonator corresponding to a sinusoidal input. During the time interval of peak to peak, the shuttle travels $4 \mu \mathrm{m}$, or $2 \mu \mathrm{m}$ shuttle displacement for peak to valley (or valley to peak). Since the optical output signal is no longer in proportion to the position signal, before any control effort can be performed on this kind of device, an optical-position signal converter has to be developed to convert the optical signal to a displacement signal. 


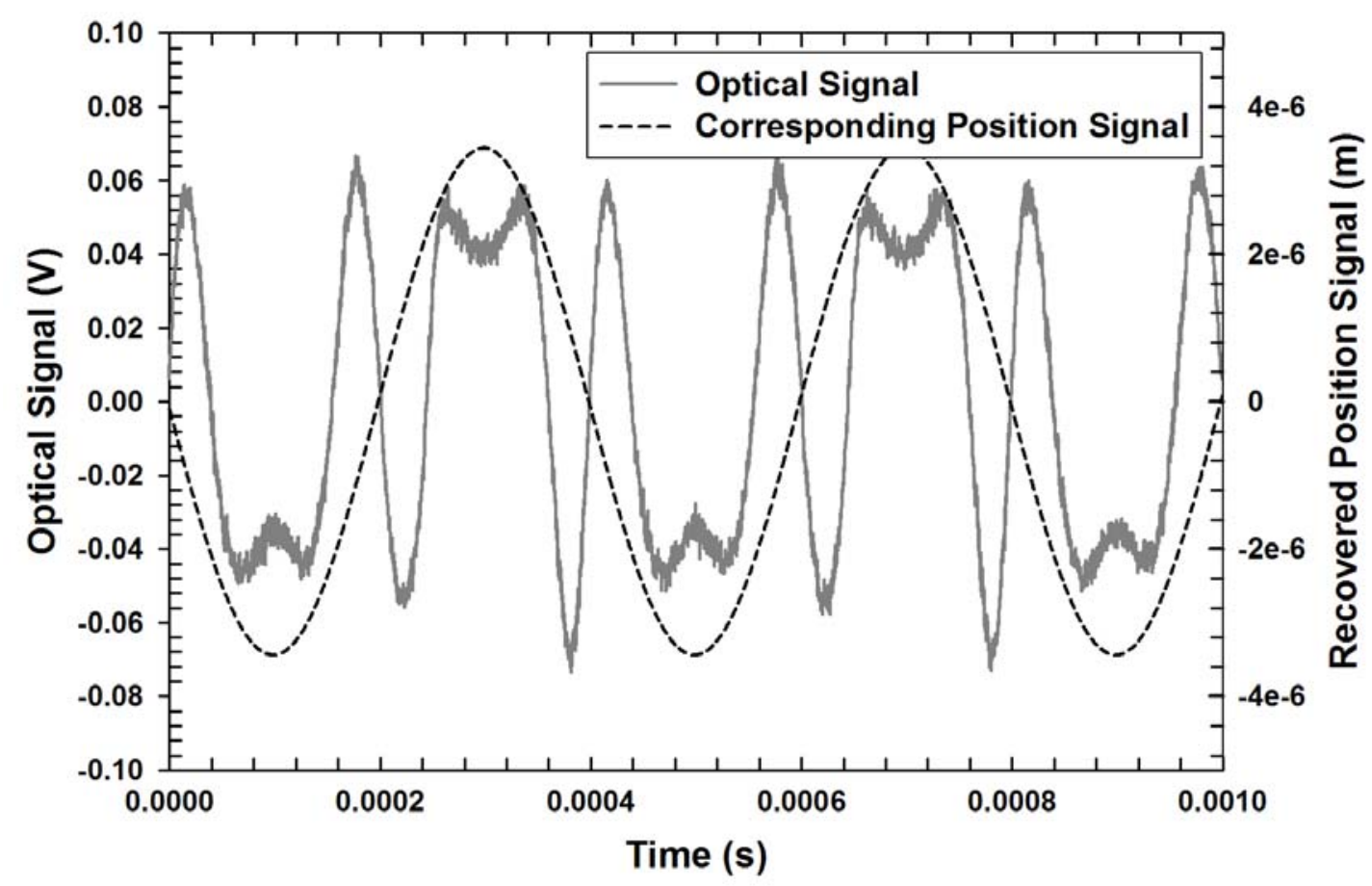

Figure 5-1: Optical output of grating structure comb resonator with its corresponding position signal

\subsection{Reconstruction Position Signal from Optical Signal}

An off-line manual position signal recovery method was developed based on the fact that the shuttle travels $2 \mu \mathrm{m}$ in the time interval of peak to valley (or valley to peak) of the optical signal. With this method, when the input to the LCR is purely sinusoidal, the position signal can be recovered based on the assumption that the position signal is also purely sinusoidal. With the position signals recovered by this method, a precise system model can be built up based on the frequency sweeping method described in chapter 3. This method is only appropriate for perfect sinusoidal signals with a peak-topeak value greater than $2 \mu \mathrm{m}$. An on-line position recovery method is necessary for the implementation of real time tracking control on the lateral comb resonator. 


\subsubsection{On-line position signal reconstruction approach \#1}

This is a model reference method. A good system model has to be built to send out set and clear instructions to the signal converter, and the model used here is built in the same way as we described in Chapter 3.

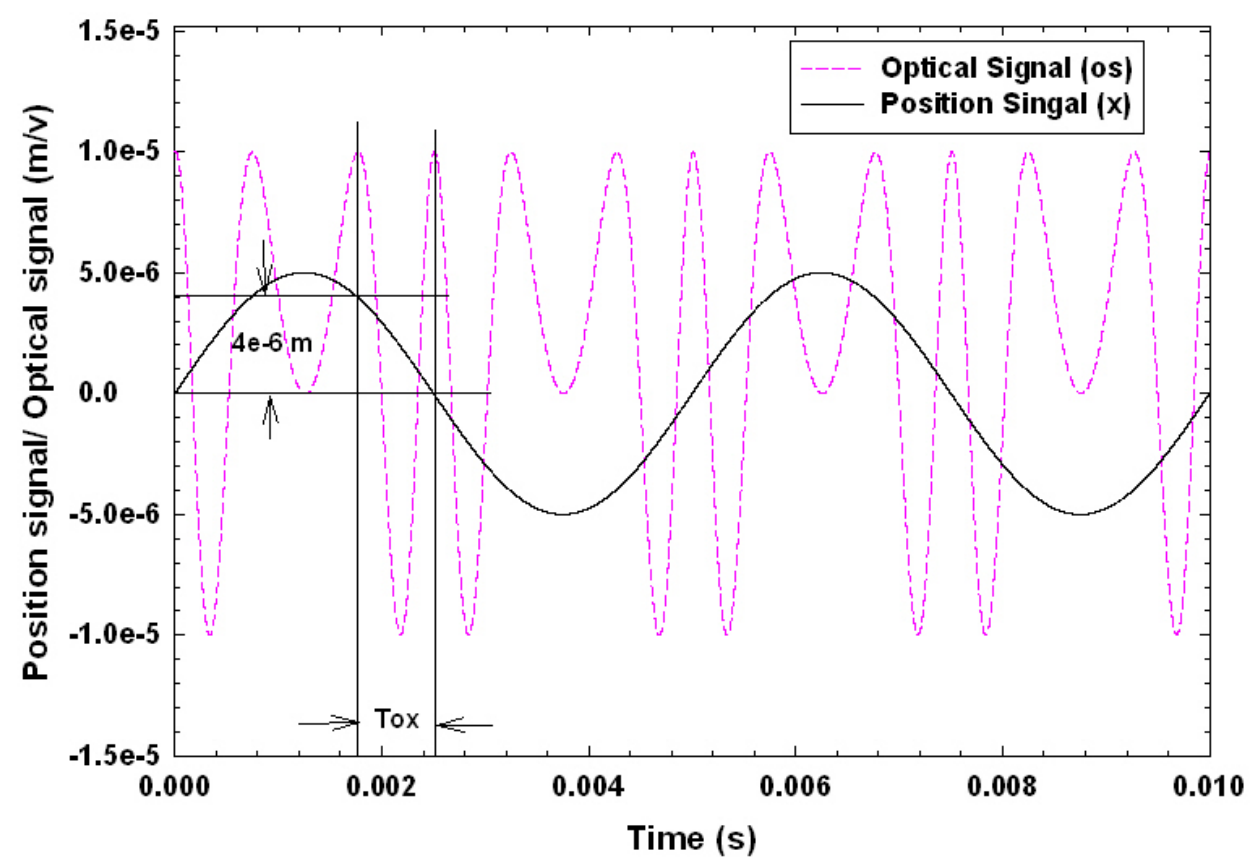

Figure 5-2: Simulated optical signal and position signal

The ideal Optical signal $\left(S_{o}\right)$ is a variation of a sinusoidal signal, the only difference between $S_{o}$ and a regular sinusoidal signal is that the frequency of the $S_{o}$ is not constant. Assume that $d t$ is a time period which is small enough so that the frequency of $S_{o}$ can be considered as a constant during $d t$. If we have $S_{o}(0)=0$, and $\phi(0)=0$, then we can get

$S_{o}(d t)=A \cos (\omega(d t) \cdot d t)$.

For the second $d t$ period, $\phi(d t)=\omega(d t) \cdot d t$, and

$S_{o}(2 d t)=A \cos (\omega(2 d t) \cdot d t+\omega(d t) \cdot d t)$,

and so on, 
then the optical signal can be finally expressed as

\section{Equation 5-1}

$S_{o}(t)=A \cos (\omega(t) \cdot d t+\omega(t-d t) \cdot d t+\cdots+\omega(d t) \cdot d t)=A \cos \left(\int_{0}^{t} \omega(t) d t\right)$,

where $A$ is the amplitude of optical signal, which can be obtained by performing peak detection on the optical signal, and $\omega(t)$ can be found by the following calculation. By observing Figure 5-2, we find that during time $T_{o x}, x$ changes $4 \times 10^{-6} \mathrm{~m}$, and during time $T_{s}(d t), x$ changes $d x$, where $T_{s}$ is the sample period. Then it can be found that

Equation 5-2 $\quad T_{o x}=\frac{4 e-6}{d x} T_{s}$.

During time interval $\left(t, t+T_{s}\right)$, the frequency of position signal is approximately

Equation 5-3 $\omega(t)=\frac{2 \pi}{T_{o x}(t)}=\frac{2 \pi}{4 e-6 T_{s}} d x=\frac{2 \pi}{4 e-6} \cdot \frac{d x}{d t}$.

Similarly, the position signal $x$ can also be deduced from the optical signal $S_{o}$. From Equation 5-1, it can be found that

Equation 5-4 $\omega(t)=\frac{d\left(\cos ^{-1}\left(\frac{S_{o}}{A}\right)\right)}{d t}$,

and from Equation 5-2, the speed signal can be found as

$$
\text { Equation 5-5 } \quad \dot{x}=\frac{d x}{d t}=\frac{4 e-6}{T_{o x}}=4 e-6 \frac{\omega(t)}{2 \pi},
$$

then the position signal $x(t)$ can be calculated as

$$
\text { Equation 5-6 } \quad x(t)=\frac{4 e-6}{2 \pi} \int \omega(t) d t=\frac{4 e-6}{2 \pi} \int\left(\frac{d\left(\cos ^{-1}\left(\frac{S_{o}}{A}\right)\right)}{d t}\right) d t .
$$

The procedure of the on-line auto recover method is shown in Figure 5-3, and a simulation of this method was implemented to verify the performance of this method. 


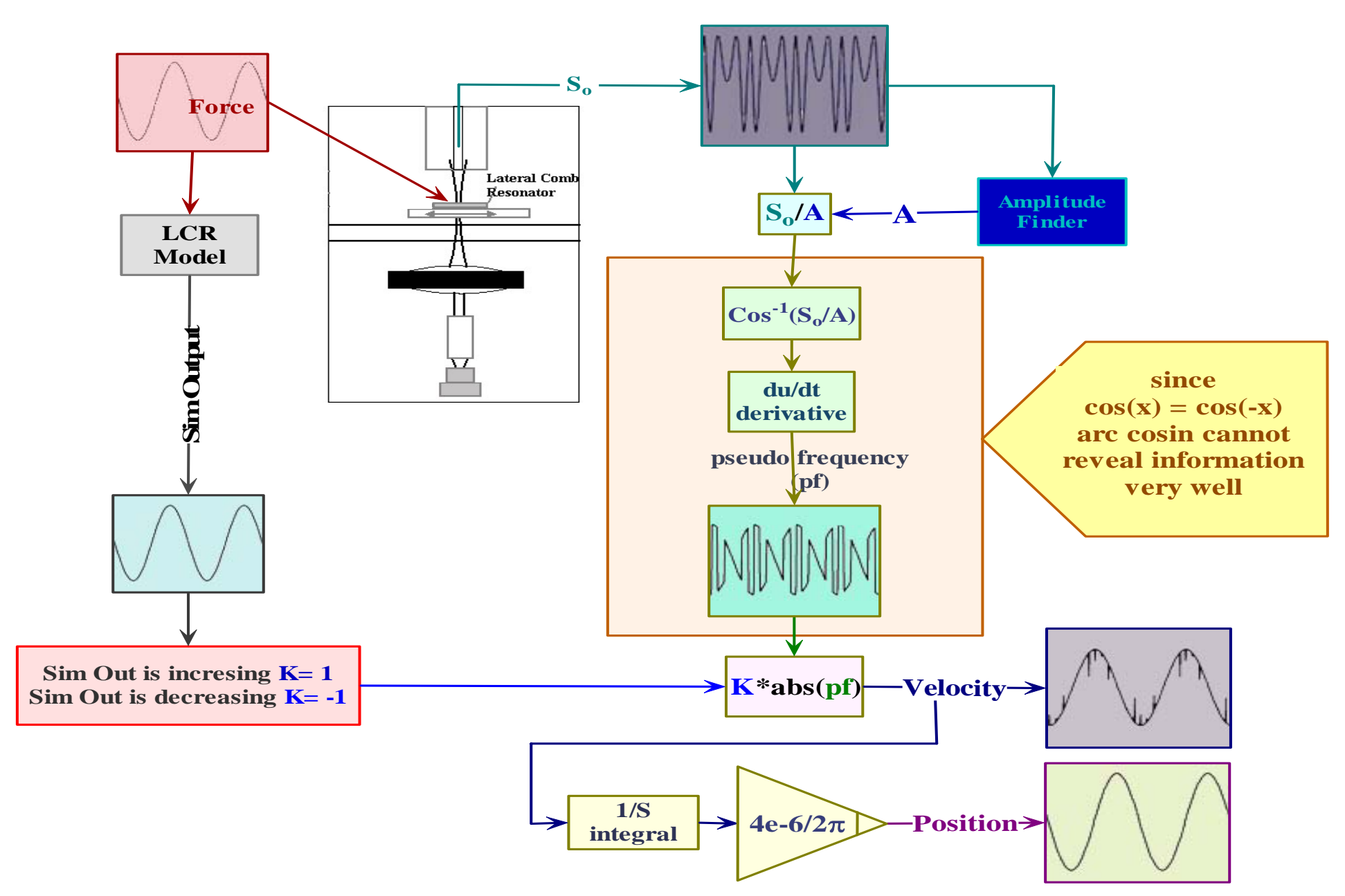

Figure 5-3: Procedure of in-line decode of optical signal 
By applying Equation 5-4 to the simulated optical signal, a pseudo frequency signal (Figure 5-4), instead of a sinusoidal frequency signal, will be achieved. This is caused by the arcsine function involved in this equation. By plotting the absolute value of the pseudo frequency (APS) together with the position signal (Figure 5-5), it is found that by flipping the sign of APS when the position signal is decreasing, the frequency signal can be established as shown in Figure 5-6.

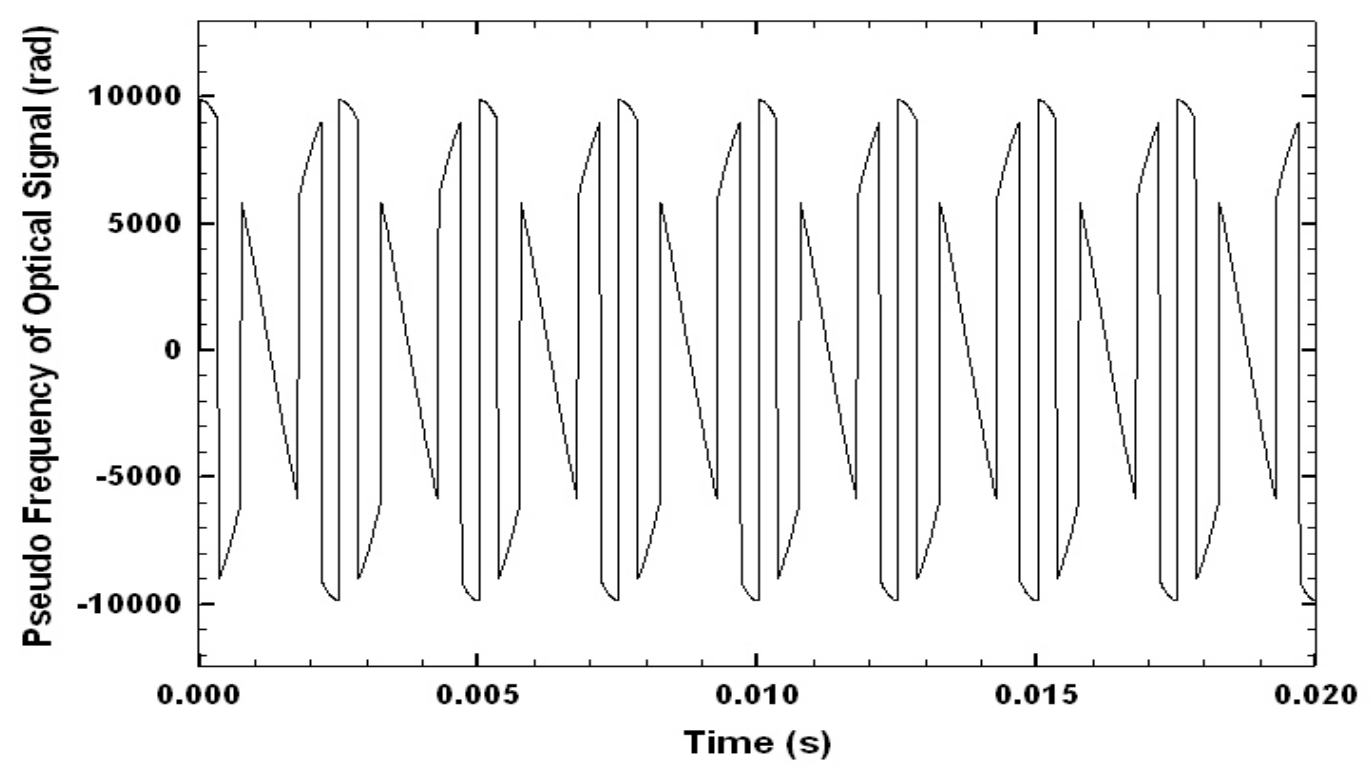

Figure 5-4: Pseudo Frequency obtained from Equation 5-4 


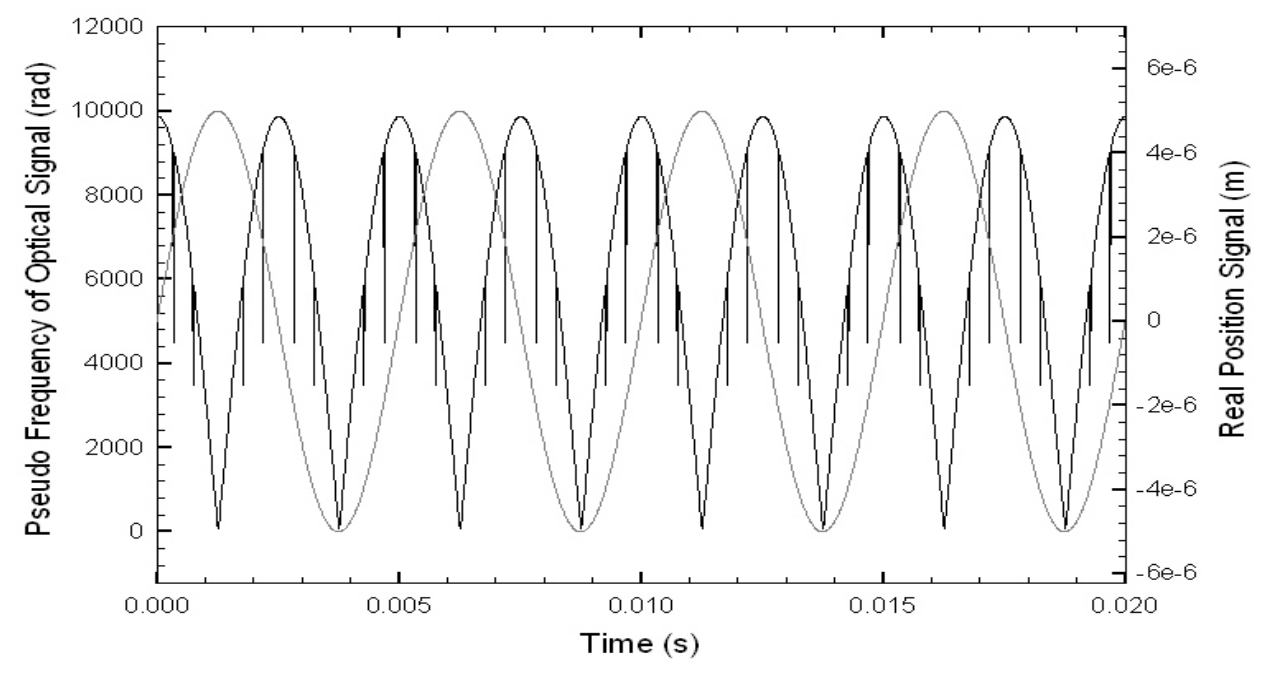

Figure 5-5: The absolute value of pseudo frequency obtained from Equation 5-4 directly

In actual implementation, a simultaneously simulated position signal was used to determine when the value of the pseudo frequency needs to be flipped. With this reconstructed frequency signal and Equation 5-5 \& Equation 5-6, the velocity and position signal can be recovered from the optical signal (Figure 5-7).

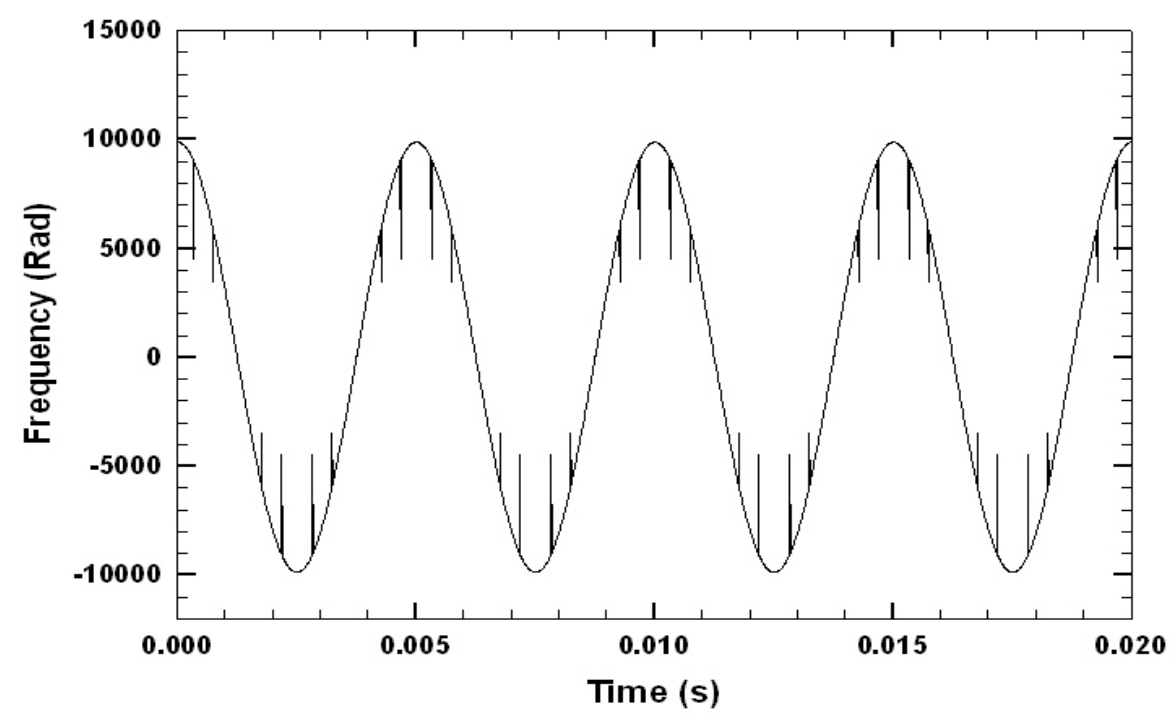

Figure 5-6: Restored Frequency of Position Signal 

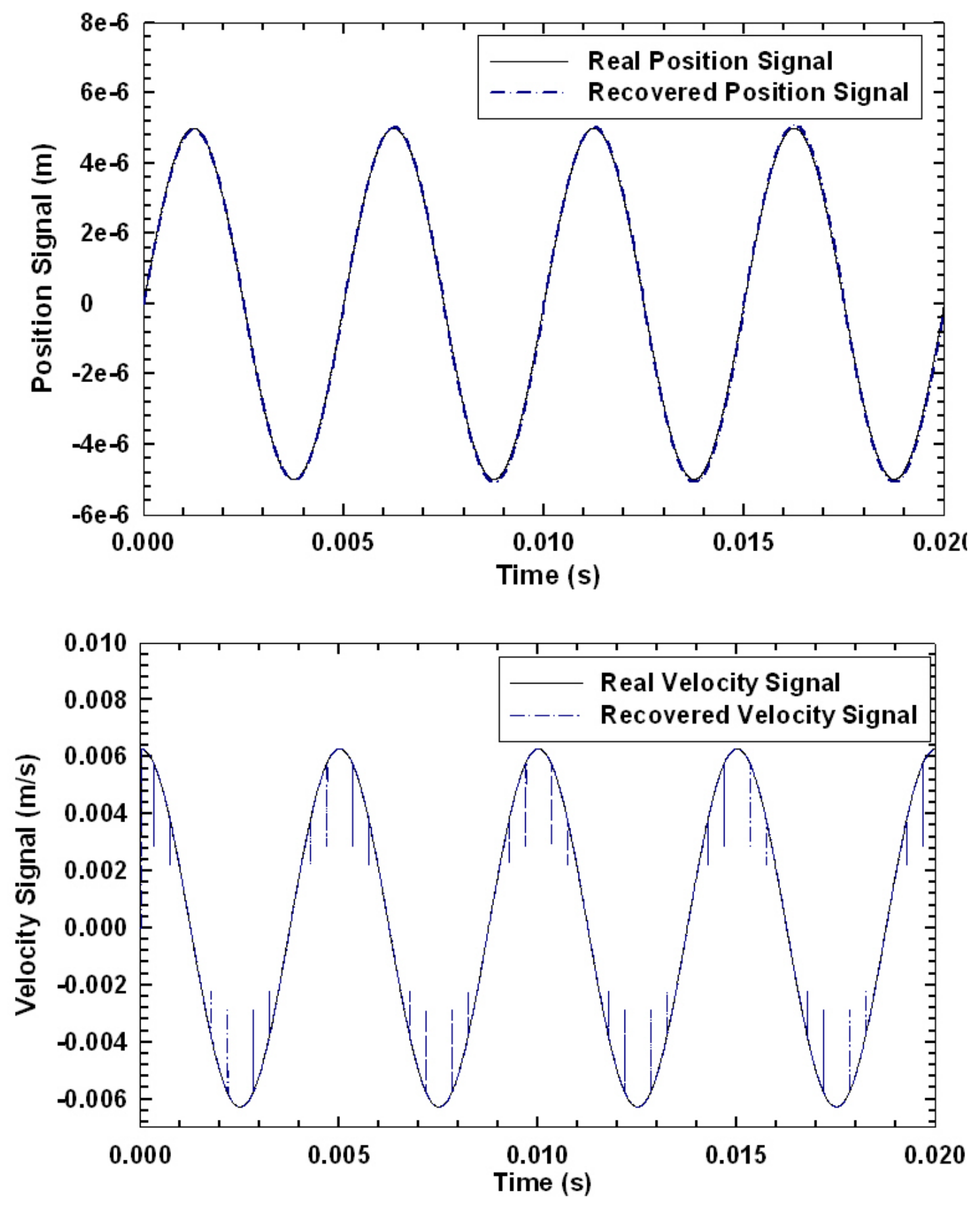

Figure 5-7: Recovered Position and Velocity Signal 
Examination of the experimental optical data (Figure 5-8) shows that a high level of noise exits in the signal. Therefore, in order to ensure the functionality of the auto-recovery algorithm for the actual optical signal, additional simulations which incorporate a noise-corrupted signal are necessary. The recovery results for the noisecorrupted signal are shown in Figure 5-9, which shows that the integral function in Equation 5-6 caused the accumulation of the noises that exist in the optical signal. This is not ideal for the signal conversion.

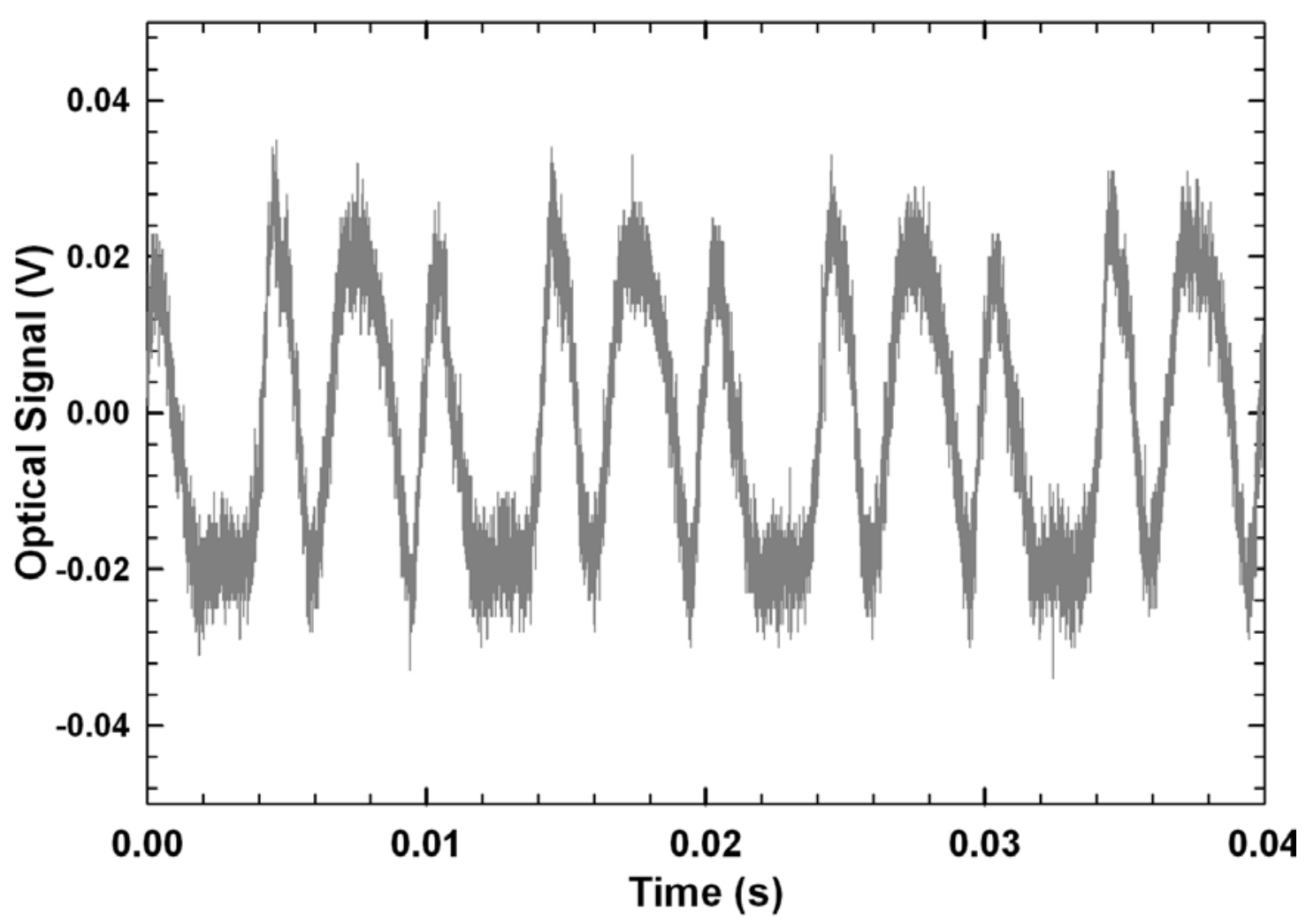

Figure 5-8: Original optical signal recorded by oscilloscope, the force frequency is $100 \mathrm{~Hz}$ 

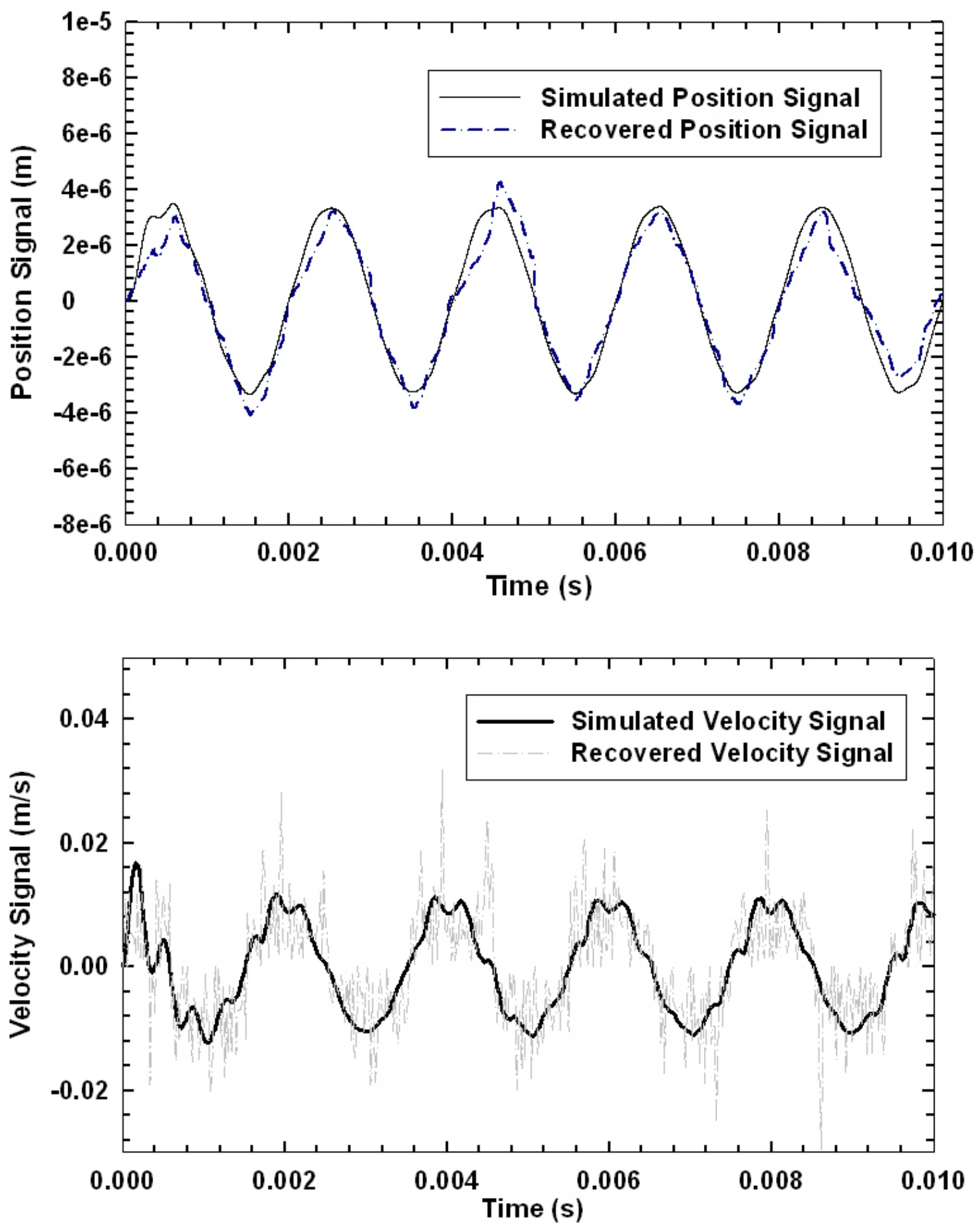

Figure 5-9: Position \& velocity Signal Recovered with on-line approach \#1 


\subsubsection{On-line position signal reconstruction approach \#2}

Both position and velocity signals can be reconstructed in real time with the on-line recovery approach described in section 5.1.1. However, as shown in Figure $5-9$, the noise present in the optical signal appears and is even amplified in the reconstructed signal. An auto-recover method, which can decouple the optical noise from the reconstructed position signal, would be a better choice. Based on this consideration, an on-line signal recovery method that deals with the optical signal's $2 \mu \mathrm{m}$ resolution directly has been surveyed.

As shown in Figure 5-10, this model referenced reconstruction method is being implemented in 3 steps.

The first step is to detect the peaks and valleys of both the optical signal and model's position signal in real time. The rough position signal will be reconstructed in the $2^{\text {nd }}$ step. At the beginning, when the first zero cross value is detected from the model's position output, the reconstructed position signal is set to zero. After that, each time when there is a peak/valley of optical signal being detected, $2 \mu \mathrm{m}$ will be added to the position signal when the output is increasing, and $2 \mu \mathrm{m}$ will be subtracted from the position signal when the output is decreasing. In the final step, the extra data in the reconstructed signal is removed. In the optical signal, usually during the peak to valley (or valley to peak) time period, the shuttle travels $2 \mu \mathrm{m}$, however, there are also some exceptions. Each time when a peak/valley occurs on the shuttle's movement, a peak/valley will also exist in the optical signal, and the shuttle movement during the peak to valley (or valley to peak) time period is not necessarily $2 \mu \mathrm{m}$. Therefore, the recovered values at those points might not be correct, and they need to be removed.

The implementation of this recovery method on an optical signal (Figure 5-11) recorded by an oscilloscope shows a good reconstruction result (Figure 5-12). Unlike the previous on-line reconstruction result, the noises that exist in the optical signal have been decoupled from the reconstructed position signal. 


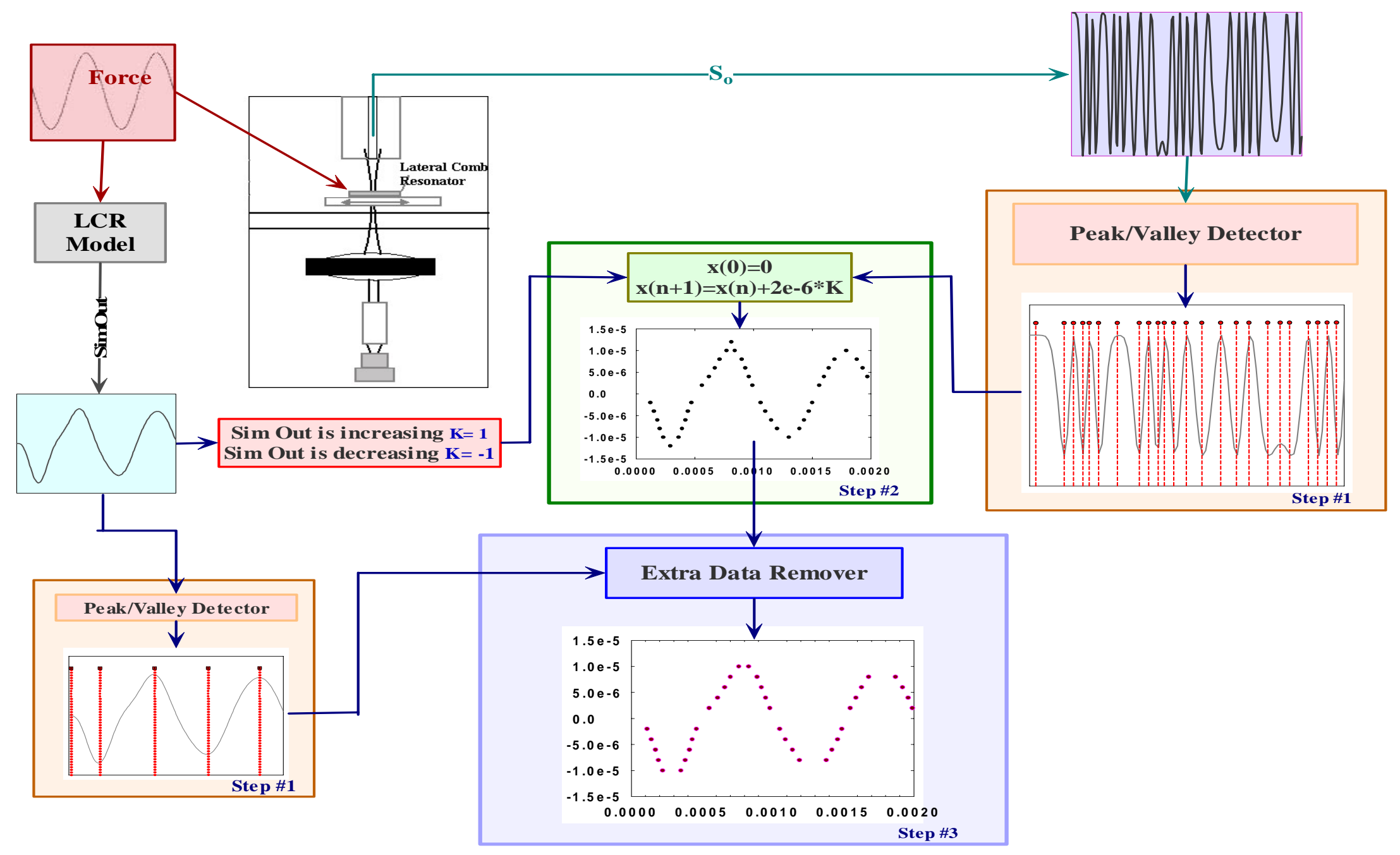

Figure 5-10 Procedure of on-line position signal reconstruction method \#2 


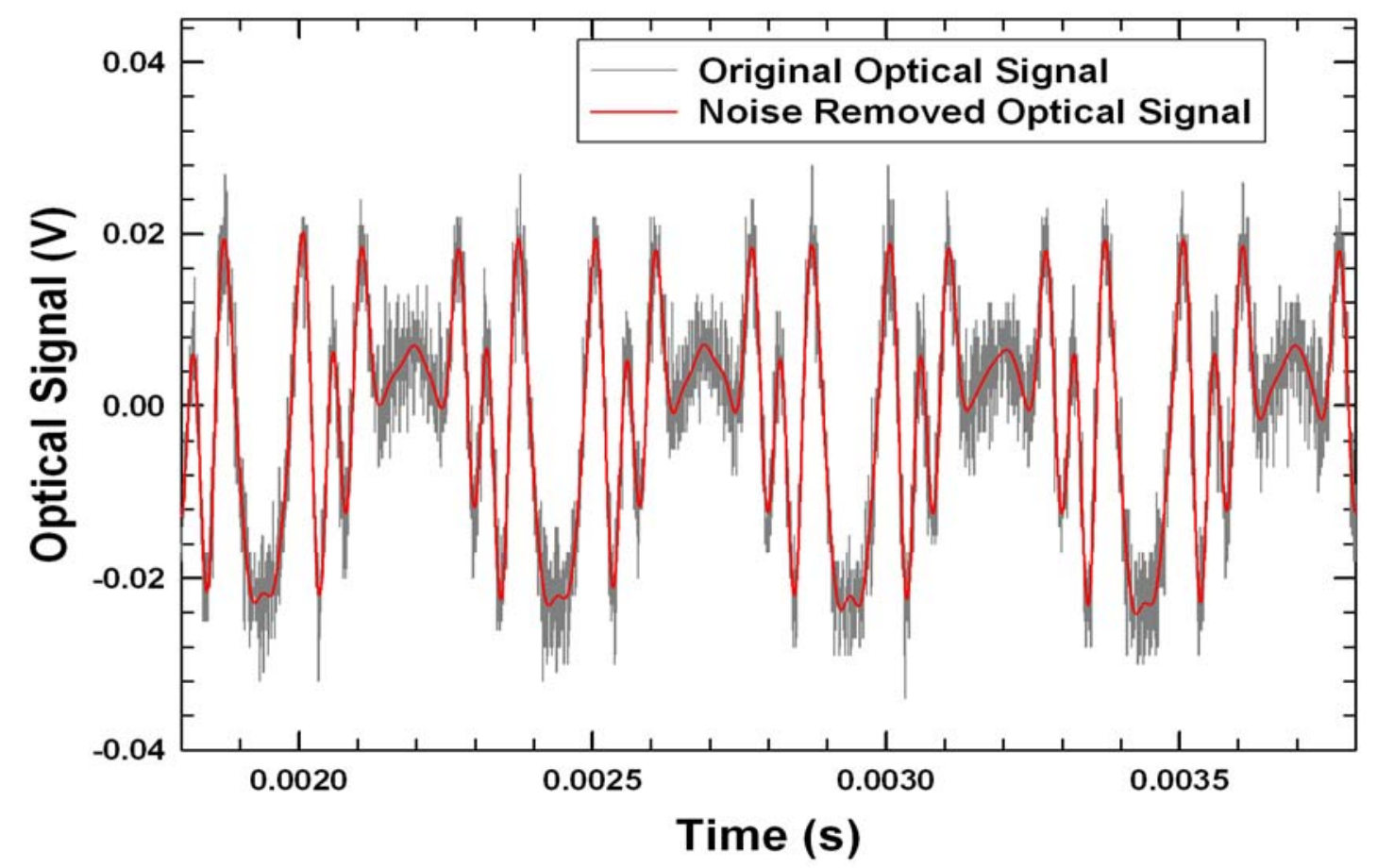

Figure 5-11: Original optical signal recorded by oscilloscope, the force frequency is $2000 \mathrm{~Hz}$

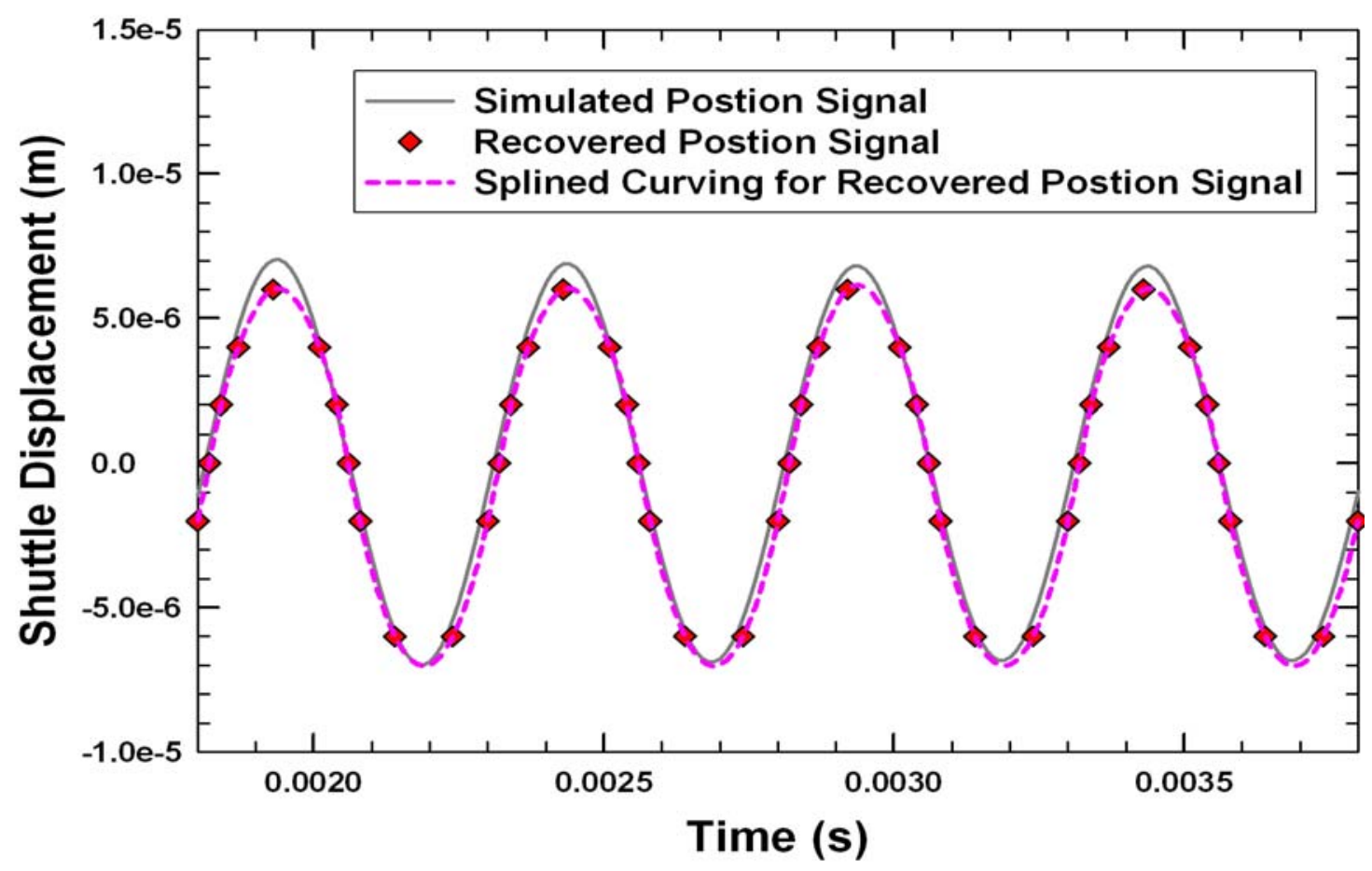

Figure 5-12 Position Signal Recovered with In-line approach \#2 
Currently, the resolution for this reconstruction method is only 2 micrometers, while the maximum displacement of this type of the LCR shuttles is $15 \mu \mathrm{m}$. Fortunately, this $2 \mu \mathrm{m}$ 's limitation will be removed soon, and this optical noise decoupled on-line position signal reconstruction method can be finally realized in the near future. With the current single beam optical observing setup, the resolution is limited by the $2 \mu \mathrm{m}$ 's fabrication feature of MUMPS technique. Even though the fabrication feature cannot be improved in a short time, resolution of this reconstruction method can still be improved several times with the implementation of multi-beam optical observing techniques $[76,77]$.

\subsection{Experimental Estimation of Electrical Force}

The electrostatic force that functioned on the LCR can be estimated by means of the sliding-mode method. The electrostatic force equation (Equation 3-2) is developed based on some assumptions and simplification. In the real world, the electrostatic force that is generated by the overlapped fingers is more complicated [78] than this force equation. Furthermore, the electrostatic force might not be a perfect sinusoidal signal when the voltage applied to the stage is a pure sine wave signal.

In this section, an experiment has been established to estimate the lateral direction electrostatic force of the LCR, and the estimation result will be compared with the Maxwell simulation result.

For the MEMS LCR system (Equation 4-1), when the load is zero, the estimated dynamic is suggested as

Equation 5-7 $\left\{\begin{array}{l}\dot{\hat{x}}_{1}=\hat{x}_{2} \\ \dot{\hat{x}}_{2}=-\frac{2 k_{s}}{m} \hat{x}_{1}-\frac{\beta}{m} \hat{x}_{2}+\frac{1}{m} \hat{F}_{e}\end{array}\right.$.

And the electrostatic force is estimated with the sliding-mode method as follows

Figure 5-13

$$
\hat{F}_{e}=\frac{V_{b} V_{s} S}{m} \operatorname{sgn}\left(V_{s}\right) \text {. }
$$


where $V b$ is the DC drive voltage, and $V s$ is the AC drive voltage. The switching line of this sliding mode estimator is defined as $x_{1}-\hat{x}_{1}$, in order to get the best performance, a PID controller is applied along the switching line, which is defined by

Equation 5-8

$$
S=K_{p} \cdot f_{n}\left(\bar{x}_{1}\right)+K_{i} \int f_{n}\left(\bar{x}_{1}\right)+K_{d} \frac{d f_{n}\left(\bar{x}_{1}\right)}{d t}
$$

where $\bar{x}_{1}=x_{1}-\hat{x}_{1}$, and the function $f_{n}(\varsigma)$ is given by, $f_{n}(\varsigma)=\frac{\varsigma}{\alpha+a b s(\varsigma)}$, where $\alpha$ is a small positive number. By substituting the sgn function for above function, the high frequency components caused by the back and forward switching commands can be reduced dramatically.

The block diagram for this force estimation experiment is shown in Figure 5-14. During the force estimation, as shown in Figure 5-15, sinusoidal voltage Vs (20V p-p) with frequency of $2000 \mathrm{~Hz}$ was applied to the shuttle, while $\pm 10 \mathrm{~V}$ 's voltages were applied to the stators (side combs).

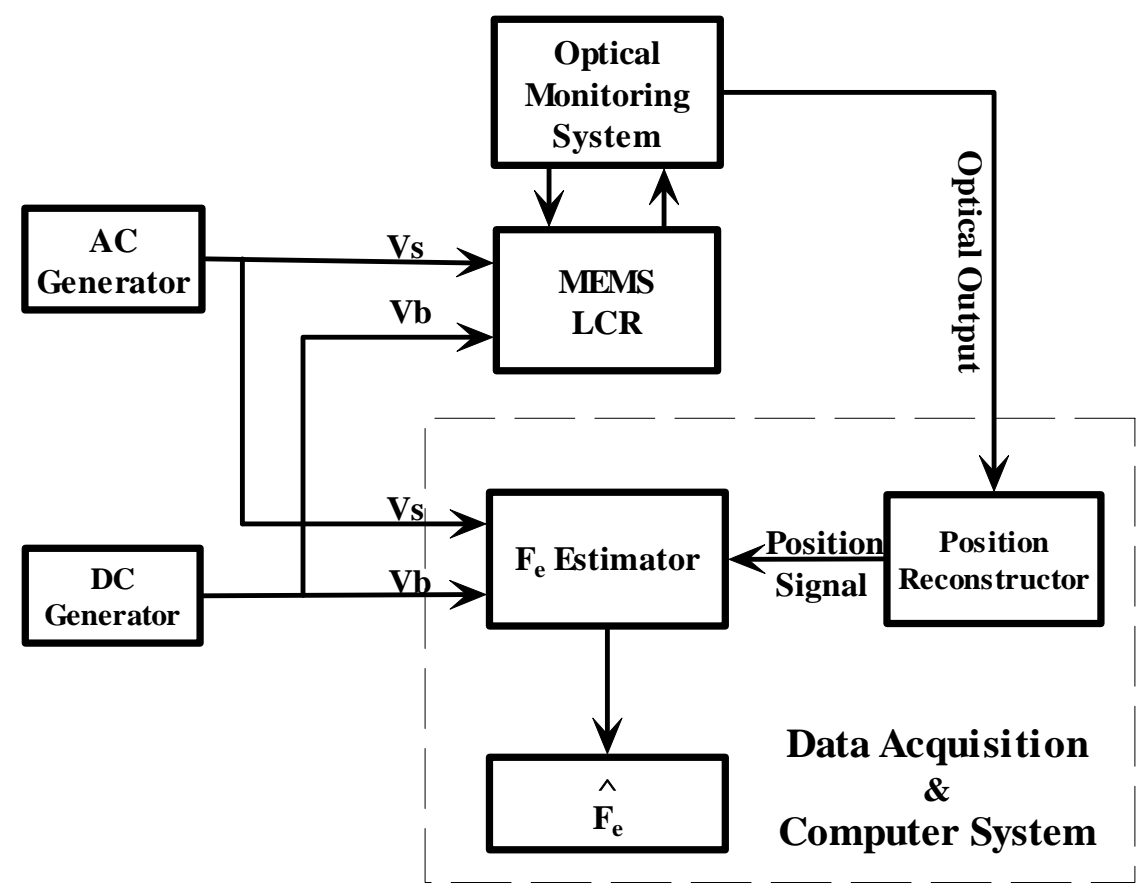

Figure 5-14: $\quad$ Block Diagram of Experimental Force Estimation

The optical observation of the shuttle displacement (Figure 5-15) was reconstructed to a position signal (Figure 5-16) in the 'Position Reconstructor' module 
with the on-line recovery approach \#1 described in section 5.1. Finally the electrostatic force was estimated within the 'Fe Estimator' module with the sliding mode based algorithm, and is plotted in Figure 5-17.

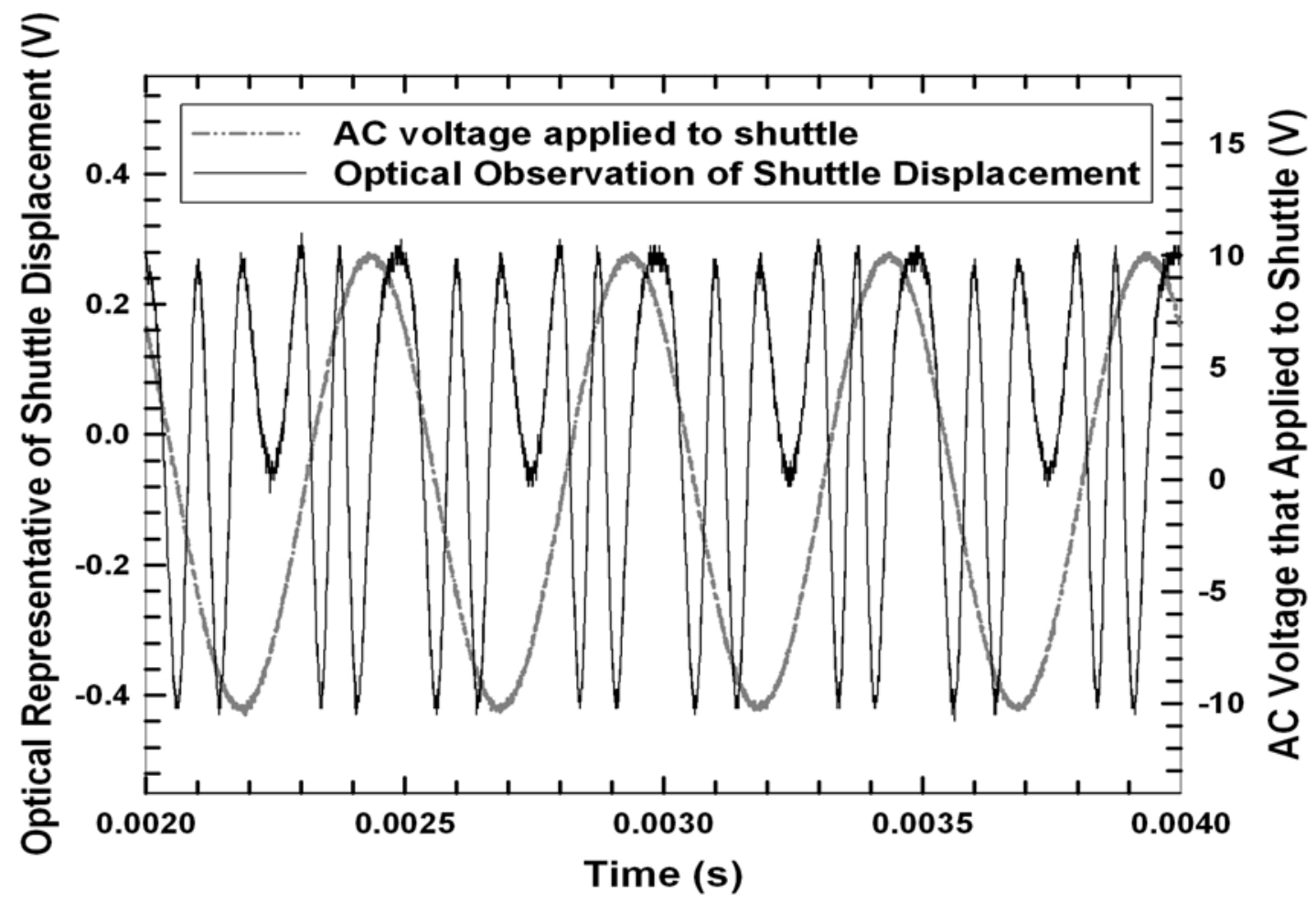

Figure 5-15: AC voltage that applied to LCR's shuttle and its corresponding optical signal 


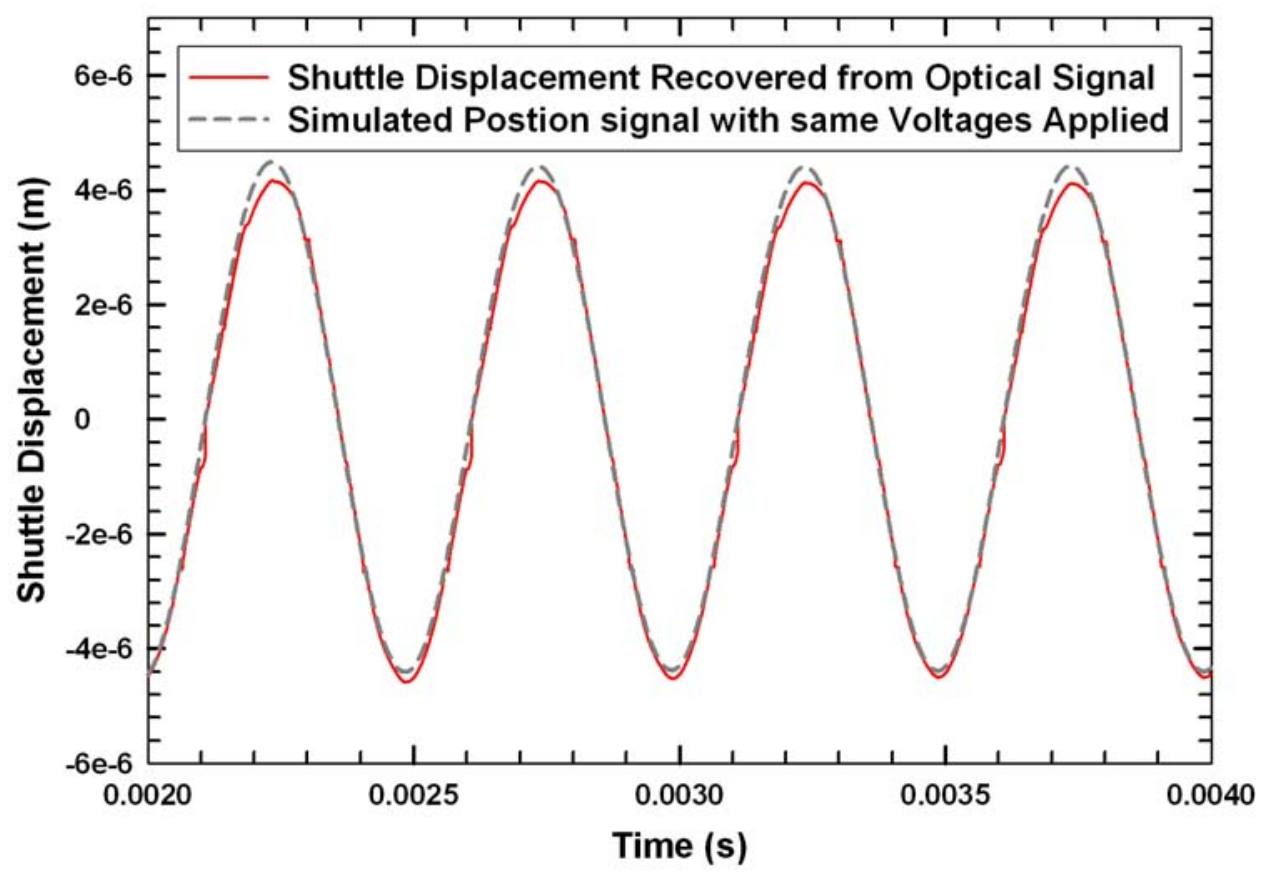

Figure 5-16: Reconstructed Shuttle Displacement and simulated position signal with same voltages input

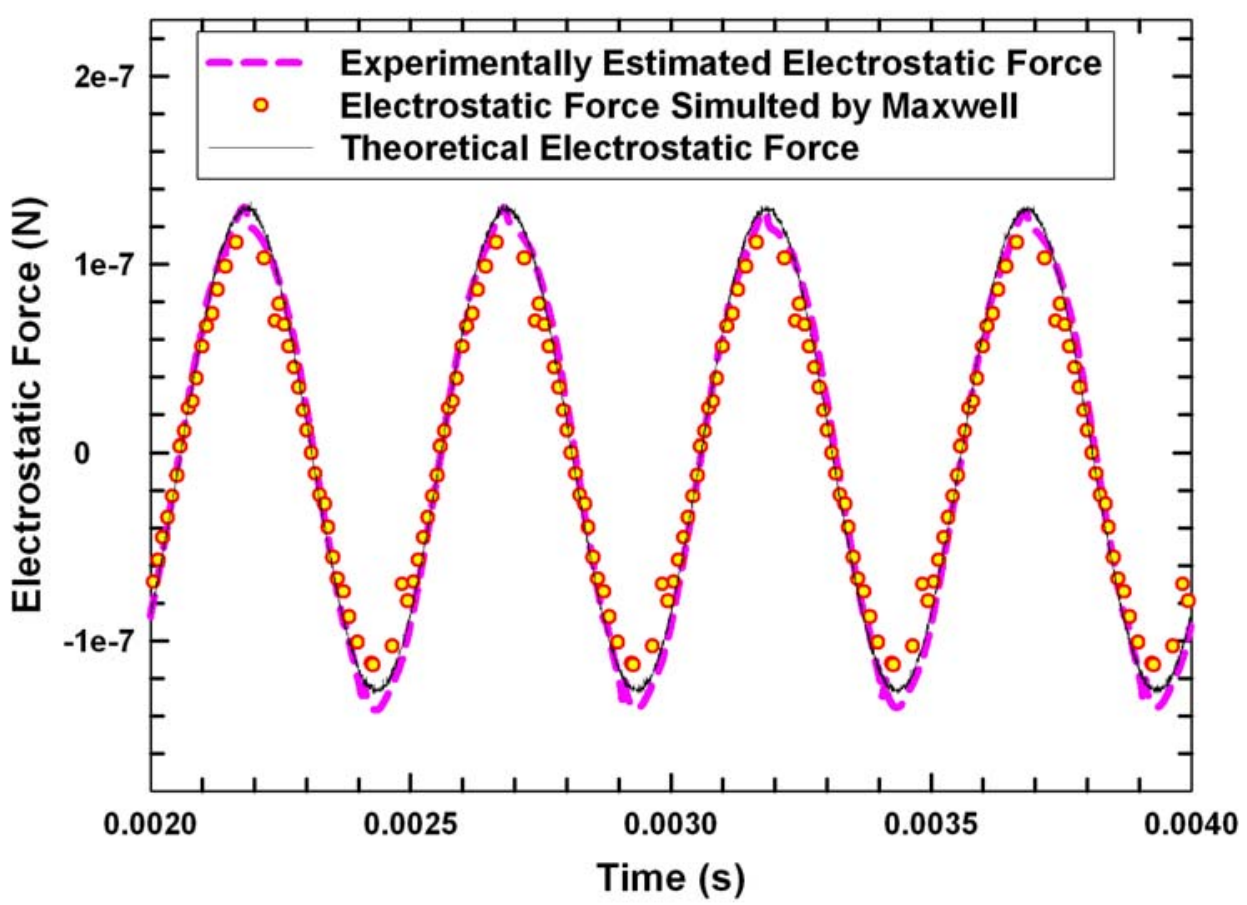

Figure 5-17: $\quad$ Experimental estimated electrostatic force and simulated electrostatic force with Ansoft's 2D Maxwell software compared with the theoretical electrostatic force. 
Ansoft's 2D Maxwell has been used to simulate the electrostatic force. In the simulation, as shown in Figure 5-18, a variable C1 has been defined as the shuttle position input of the model. For example, when $\mathrm{C} 1=60 \mu \mathrm{m}$, that means the shuttle is in its zero position, and when $\mathrm{C} 1=62 \mu \mathrm{m}$, the shuttle is $2 \mu \mathrm{m}$ off its zero position. As shown in Table 5-1, the simulation has been performed 41 times to simulate a complete cycle of the AC input voltage, where the corresponding shuttle displacements were obtained from the experimentally reconstructed position signal. The simulated force is plotted in Figure 5-17 as well.

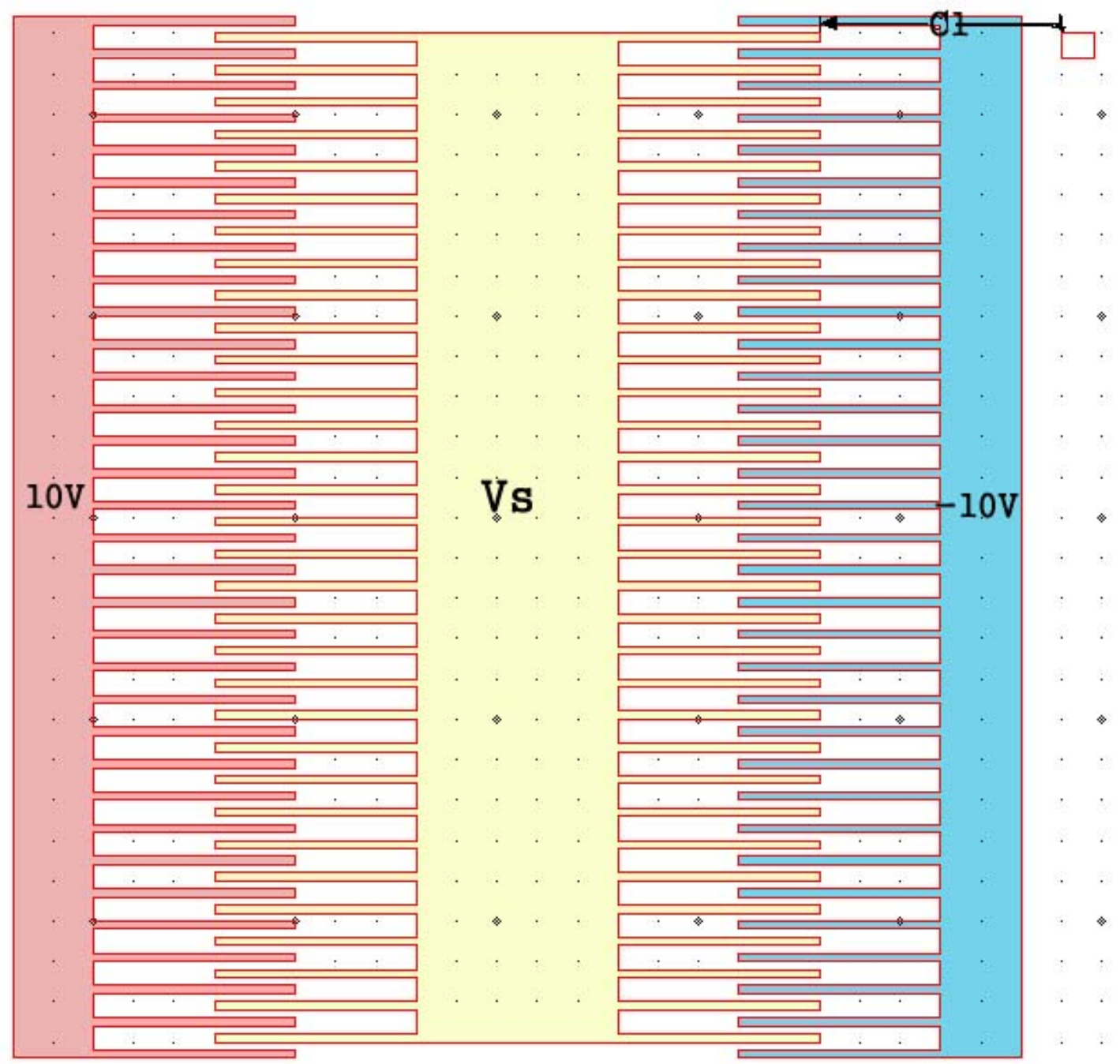

Figure 5-18: $\quad$ LCR model used in the Maxwell simulation 
Table 5-1: Input and output for Maxwell simulation

\begin{tabular}{|c|c|c|c|c|}
\hline Time (s) & Vs & $\begin{array}{c}\text { Shuttle } \\
\text { Displacement (m) }\end{array}$ & C1 (m) & Fx (N) \\
\hline $2.4280 \mathrm{E}-03$ & 10 & $-3.3662 \mathrm{E}-06$ & 5.6634E-05 & $1.12752 \mathrm{E}-07$ \\
\hline 2.4652E-03 & 9 & $-4.2872 \mathrm{E}-06$ & 5.5713E-05 & $1.02908 \mathrm{E}-07$ \\
\hline $2.4840 \mathrm{E}-03$ & 8 & $-4.4069 \mathrm{E}-06$ & 5.5593E-05 & 6.97894E-08 \\
\hline $2.4948 \mathrm{E}-03$ & 7 & $\begin{array}{l}-4.3649 \mathrm{E}-06 \\
\end{array}$ & 5.5635E-05 & 7.87596E-08 \\
\hline $2.5056 \mathrm{E}-03$ & 6 & $-4.2428 \mathrm{E}-06$ & 5.5757E-05 & 6.86262E-08 \\
\hline $2.5152 \mathrm{E}-03$ & 5 & $-4.0688 \mathrm{E}-06$ & $5.5931 \mathrm{E}-05$ & 5.70438E-08 \\
\hline $2.5244 \mathrm{E}-03$ & 4 & $-3.8467 \mathrm{E}-06$ & 5.6153E-05 & 4.49756E-08 \\
\hline 2.5332E-03 & 3 & $-3.5864 \mathrm{E}-06$ & $5.6414 \mathrm{E}-05$ & 3.4359E-08 \\
\hline $2.5424 \mathrm{E}-03$ & 2 & $-3.2676 \mathrm{E}-06$ & 5.6732E-05 & 2.30576E-08 \\
\hline $2.5516 \mathrm{E}-03$ & 1 & $-2.9055 \mathrm{E}-06$ & 5.7095E-05 & 1.20909E-08 \\
\hline $2.5576 \mathrm{E}-03$ & 0 & $-2.6482 \mathrm{E}-06$ & 5.7352E-05 & $-3.30742 E-09$ \\
\hline 2.5652E-03 & -1 & $-2.3010 \mathrm{E}-06$ & 5.7699E-05 & $-1.12506 \mathrm{E}-08$ \\
\hline 2.5732E-03 & -2 & $-1.9131 \mathrm{E}-06$ & 5.8087E-05 & $-2.33688 E-08$ \\
\hline 2.5812E-03 & -3 & $-1.5060 \mathrm{E}-06$ & $5.8494 \mathrm{E}-05$ & $-2.70394 \mathrm{E}-08$ \\
\hline $2.5888 \mathrm{E}-03$ & -4 & $-1.1052 \mathrm{E}-06$ & 5.8895E-05 & $-3.91524 \mathrm{E}-08$ \\
\hline 2.5992E-03 & -5 & $-5.4116 \mathrm{E}-07$ & 5.9459E-05 & $-5.61106 \mathrm{E}-08$ \\
\hline $2.6080 \mathrm{E}-03$ & -6 & $-5.6421 \mathrm{E}-08$ & 5.9944E-05 & $-6.6935 E-08$ \\
\hline 2.6192E-03 & -7 & $5.6066 \mathrm{E}-07$ & $6.0561 \mathrm{E}-05$ & $-7.34792 \mathrm{E}-08$ \\
\hline 2.6292E-03 & -8 & 1.1027E-06 & 6.1103E-05 & -8.62942E-08 \\
\hline $2.6448 \mathrm{E}-03$ & -9 & $1.9108 \mathrm{E}-06$ & $6.1911 \mathrm{E}-05$ & $-9.87152 \mathrm{E}-08$ \\
\hline $2.6648 \mathrm{E}-03$ & -10 & 2.8364E-06 & $6.2836 \mathrm{E}-05$ & $-1.11592 \mathrm{E}-07$ \\
\hline $2.7188 \mathrm{E}-03$ & -9 & 4.3261E-06 & $6.4326 \mathrm{E}-05$ & $-1.03098 \mathrm{E}-07$ \\
\hline $2.7396 \mathrm{E}-03$ & -8 & 4.4017E-06 & $6.4402 \mathrm{E}-05$ & $-6.9772 \mathrm{E}-08$ \\
\hline $2.7480 \mathrm{E}-03$ & -7 & 4.3478E-06 & $6.4348 \mathrm{E}-05$ & $-7.8796 \mathrm{E}-08$ \\
\hline $2.7576 \mathrm{E}-03$ & -6 & $4.2276 \mathrm{E}-06$ & $6.4228 \mathrm{E}-05$ & $-6.78018 \mathrm{E}-08$ \\
\hline $2.7668 \mathrm{E}-03$ & -5 & 4.0551E-06 & $6.4055 \mathrm{E}-05$ & -5.61896E-08 \\
\hline $2.7764 \mathrm{E}-03$ & -4 & 3.8179E-06 & $6.3818 \mathrm{E}-05$ & $-4.493 \mathrm{E}-08$ \\
\hline $2.7856 \mathrm{E}-03$ & -3 & 3.5388E-06 & 6.3539E-05 & -3.43092E-08 \\
\hline 2.7936E-03 & -2 & 3.2579E-06 & $6.3258 \mathrm{E}-05$ & $-2.21428 \mathrm{E}-08$ \\
\hline $2.8008 \mathrm{E}-03$ & -1 & 2.9772E-06 & $6.2977 \mathrm{E}-05$ & $-1.14806 \mathrm{E}-08$ \\
\hline 2.8092E-03 & 0 & 2.6194E-06 & 6.2619E-05 & 8.78478E-11 \\
\hline 2.8172E-03 & 1 & $2.2517 \mathrm{E}-06$ & $6.2252 \mathrm{E}-05$ & 1.10712E-08 \\
\hline $2.8256 \mathrm{E}-03$ & 2 & $1.8416 \mathrm{E}-06$ & $6.1842 \mathrm{E}-05$ & 2.24402E-08 \\
\hline 2.8352E-03 & 3 & 1.3483E-06 & $6.1348 \mathrm{E}-05$ & 2.72246E-08 \\
\hline $2.8416 \mathrm{E}-03$ & 4 & $1.0083 \mathrm{E}-06$ & $6.1008 \mathrm{E}-05$ & 3.96352E-08 \\
\hline 2.8512E-03 & 5 & 4.8701E-07 & 6.0487E-05 & 5.55728E-08 \\
\hline $2.8596 \mathrm{E}-03$ & 6 & $2.5062 \mathrm{E}-08$ & $6.0025 \mathrm{E}-05$ & 6.7063E-08 \\
\hline 2.8712E-03 & 7 & $-6.1205 \mathrm{E}-07$ & $5.9388 \mathrm{E}-05$ & 7.38484E-08 \\
\hline 2.8824E-03 & 8 & $-1.2147 \mathrm{E}-06$ & $5.8785 \mathrm{E}-05$ & 8.73162E-08 \\
\hline $2.8980 \mathrm{E}-03$ & 9 & $-2.0110 \mathrm{E}-06$ & 5.7989E-05 & $1.00943 \mathrm{E}-07$ \\
\hline $2.9248 \mathrm{E}-03$ & 10 & $-3.1826 \mathrm{E}-06$ & 5.6817E-05 & $1.12084 \mathrm{E}-07$ \\
\hline
\end{tabular}


In theory, the electrostatic force $F_{e}$ can be calculated with Equation 3-2, and the result has also been plotted in Figure 5-17. All three results agreed with each other very well, which gives us great confidence that the electrostatic force has been properly estimated in the experiment. This success confirms the feasibility of this force estimation technique under the condition that the position signal of the MEMS device is available.

\subsection{Closed-loop Tracking Control}

The ability for tracking a certain moving path might be desirable for some applications of LCR. In this study, a fuzzy-sliding model reference adaptive controller has been designed to fulfill the tracking control task for the lateral comb resonator.

Currently, there is a limitation of the through wafer optical monitoring technique used in this project, which is, the optical signal that represents the movement of the grating structure LCR's shuttle. This signal can be reconstructed to a position signal only when the movement of the shuttle is greater than $2 \mu \mathrm{m}$. For a high performance LCR, it should have the ability to track any arbitrary patterns including those with movement less than $2 \mu \mathrm{m}$. To compensate for this limitation, a Model Reference method has been implemented.

\section{Fuzzy-Sliding Model Reference Adaptive Controller (FSMC)}

With $F_{e}$ be treated as input $\mathrm{u}$, and $F d$ be treated as the noise or disturbance, the LCR system (Equation 4-1) can be described in the following state space format, where

$$
\begin{gathered}
A=\left[\begin{array}{cc}
0 & 1 \\
-\frac{2 k_{s}}{m} & -\frac{\beta}{m}
\end{array}\right], \\
B=\left[\begin{array}{c}
0 \\
\frac{1}{m}
\end{array}\right],
\end{gathered}
$$

and

$$
C=\left[\begin{array}{ll}
1 & 0
\end{array}\right]
$$

Success in the simulation of position control with sliding mode method has been achieved by the following switching surface design [79], 
Equation 5-9 $\quad S(t)=h\left[X(t)-\int_{0}^{t}(A+B k) X(\tau) d \tau\right]=0$.

where

Equation 5-10 $\mathbf{X}=\left[\begin{array}{l}x_{1} \\ x_{2}\end{array}\right]$,

and $k$ is the linear control gain obtained by the LQR method. Then the position controller will be

$$
F_{e}=k X(t)-\bar{\beta} \operatorname{sgn}(S(t)) \text {, }
$$

where $\bar{\beta}$ is designed as the upper bound of the lumped uncertainty.

A slight modification has been made on the controller to meet the requirements of tracking control,

$$
\text { Equation 5-11 } \quad F_{e}=k \varepsilon(t)-\bar{\beta} \operatorname{sgn}(S(t)) .
$$

where

$$
\varepsilon=\left[\begin{array}{c}
x_{1}-r \\
x_{2}-\dot{r}
\end{array}\right],
$$

and $r$ is the desired moving path that the controller will force the shuttle to track.

This sliding mode controller operates well in simulation when the outputs from the system are noiseless position and velocity signals. The performance of this sliding controller is degraded when the position and velocity signals are not directly available, especially when the noise level of the optical signal is relatively high, which consequently causes a high level of noise in the position and velocity signals that are reconstructed from the optical signal. A new model reference adaptive fuzzy sliding mode controller (MFSC) was developed based on the previous sliding mode controller to compensate for the additional imprecision introduced by the auto-recovery algorithm. The MFSC can be described as

Equation 5-12

$$
F_{e}=k \hat{\varepsilon}-\bar{\beta} \operatorname{sgn}(S)+f u z z(S)+k_{m}\left[\begin{array}{c}
x_{1}-\hat{x}_{1} \\
x_{2}-\hat{x}_{2}
\end{array}\right] .
$$


Where $\hat{x}_{1}$ and $\hat{x}_{2}$ are position and velocity outputs from the system model as shown in Figure 5-19, and

and

$$
\begin{gathered}
\hat{\varepsilon}=\left[\begin{array}{l}
\hat{x}_{1}-r \\
\hat{x}_{2}-\dot{r}
\end{array}\right], \\
\hat{S}(t)=h\left[\hat{X}(t)-\int_{0}^{t}(A+B k) \hat{X}(\tau) d \tau\right], \\
\text { fuzz }(\hat{S})= \begin{cases}k_{g}(-1+\bar{b}) & \hat{S} \geq 0 \\
k_{g}(-1-\bar{b}) & \hat{S}<0\end{cases}
\end{gathered}
$$

is the fuzzy tuner for the sliding controller.

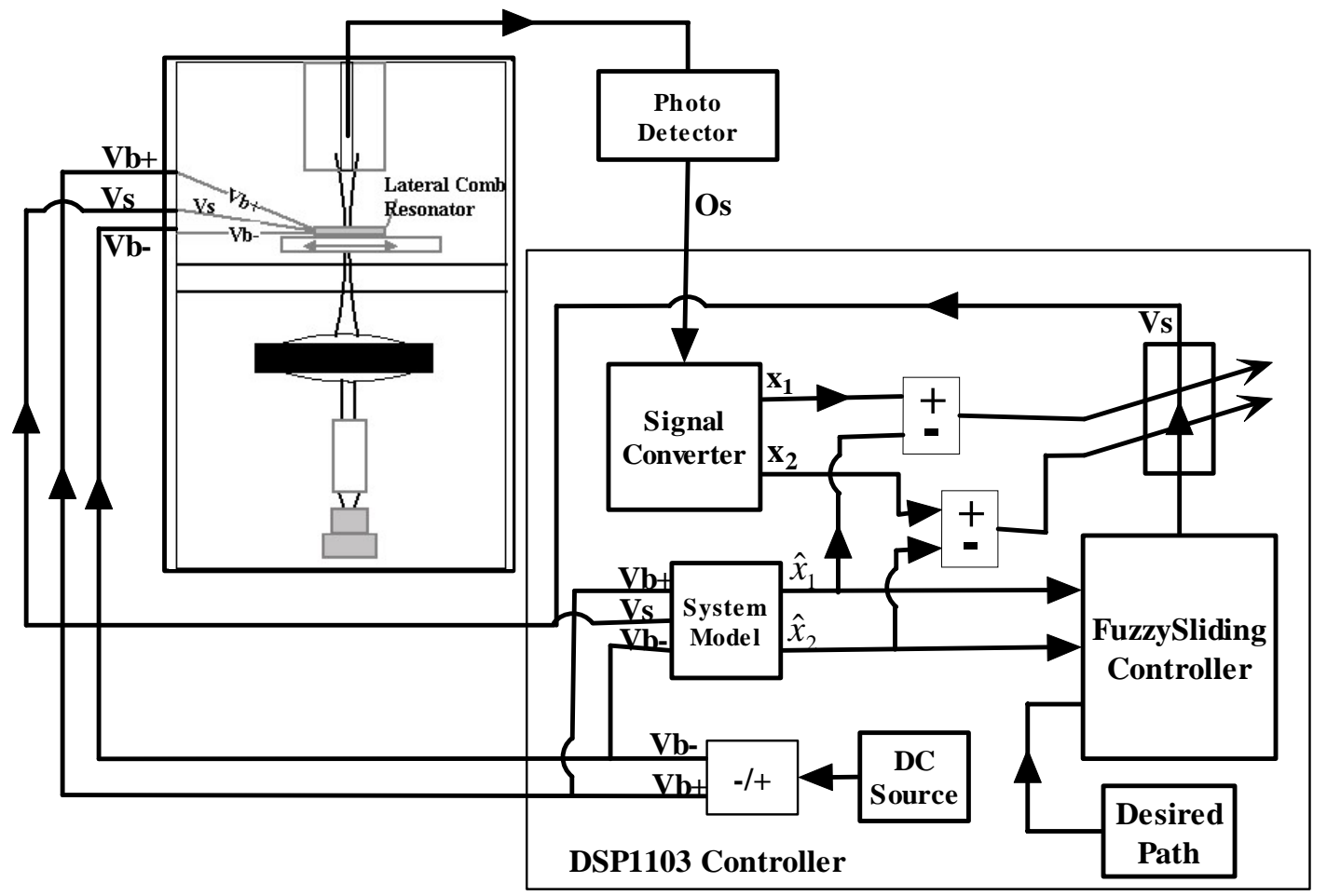

Figure 5-19: Flowchart of MFSC

Again, the through-wafer optical monitoring technique has been used in this experiment to obtain the optical signal, from which the actual shuttle position and velocity signal can be reconstructed and fed into the MFSC controller. The dSpace 
DSP1103 real time controller board is used to implement both the auto-recovery algorithm and the real time tracking control routine. The optical signal was first reconstructed into displacement and velocity signals in the so-called 'signal-converter' unit. These reconstructed signals are then fed into the MFSC controller to adjust the control signal (which is the voltage used to drive the MEMS lateral comb resonator) in the 'fuzzy sliding controller' unit. The input signals of the 'fuzzy sliding controller' are the outputs of a well-modeled reference system which behaves similar to the MEMS lateral comb resonator. The control signal will be finally adjusted based on the difference between the reconstructed position/velocity signals and the model's position/velocity outputs. Figure 5-20 and Figure 5-21 show the tracking control results. It can be seen from these plots that, although the tracking is not perfect, the shuttle can steadily follow the changes in the desired displacement.

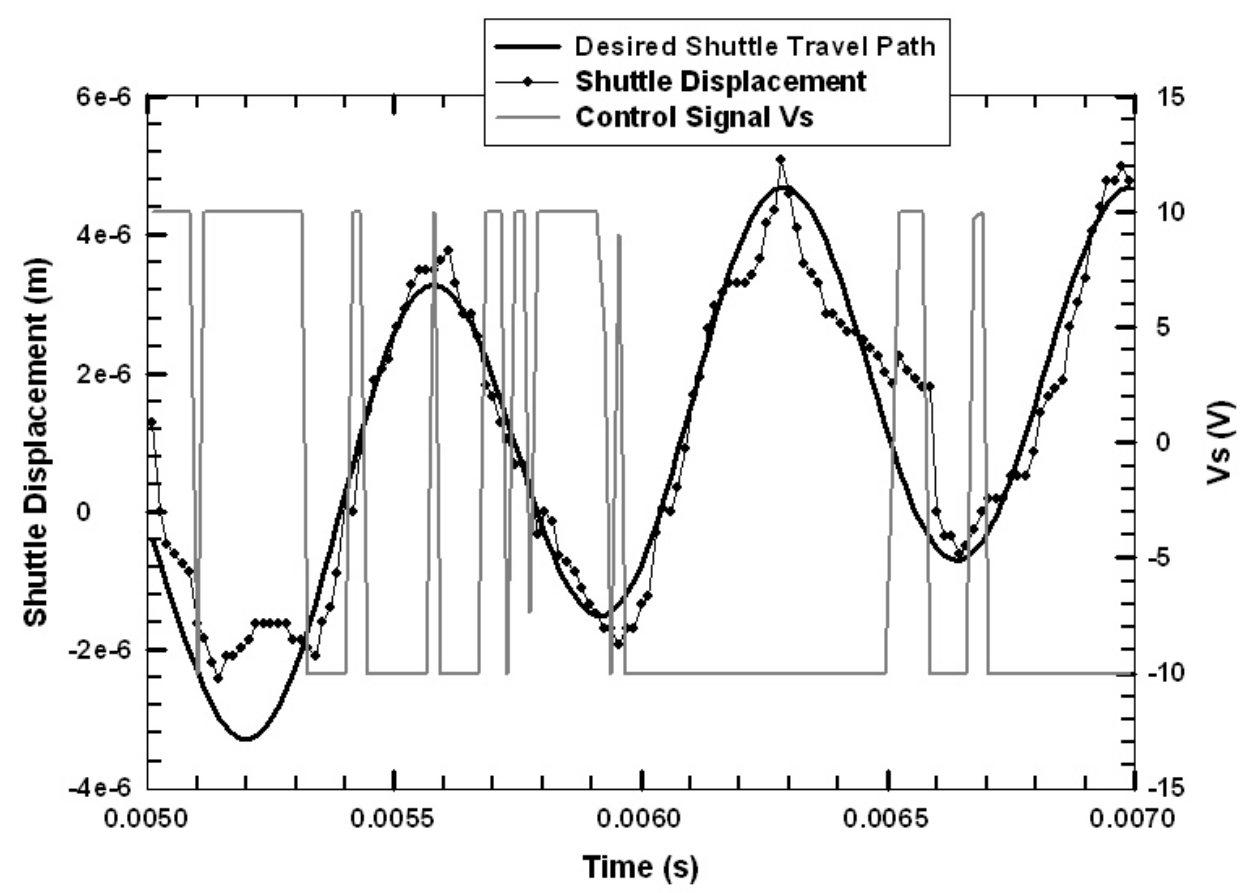

Figure 5-20: Experimental Tracking Results 1 


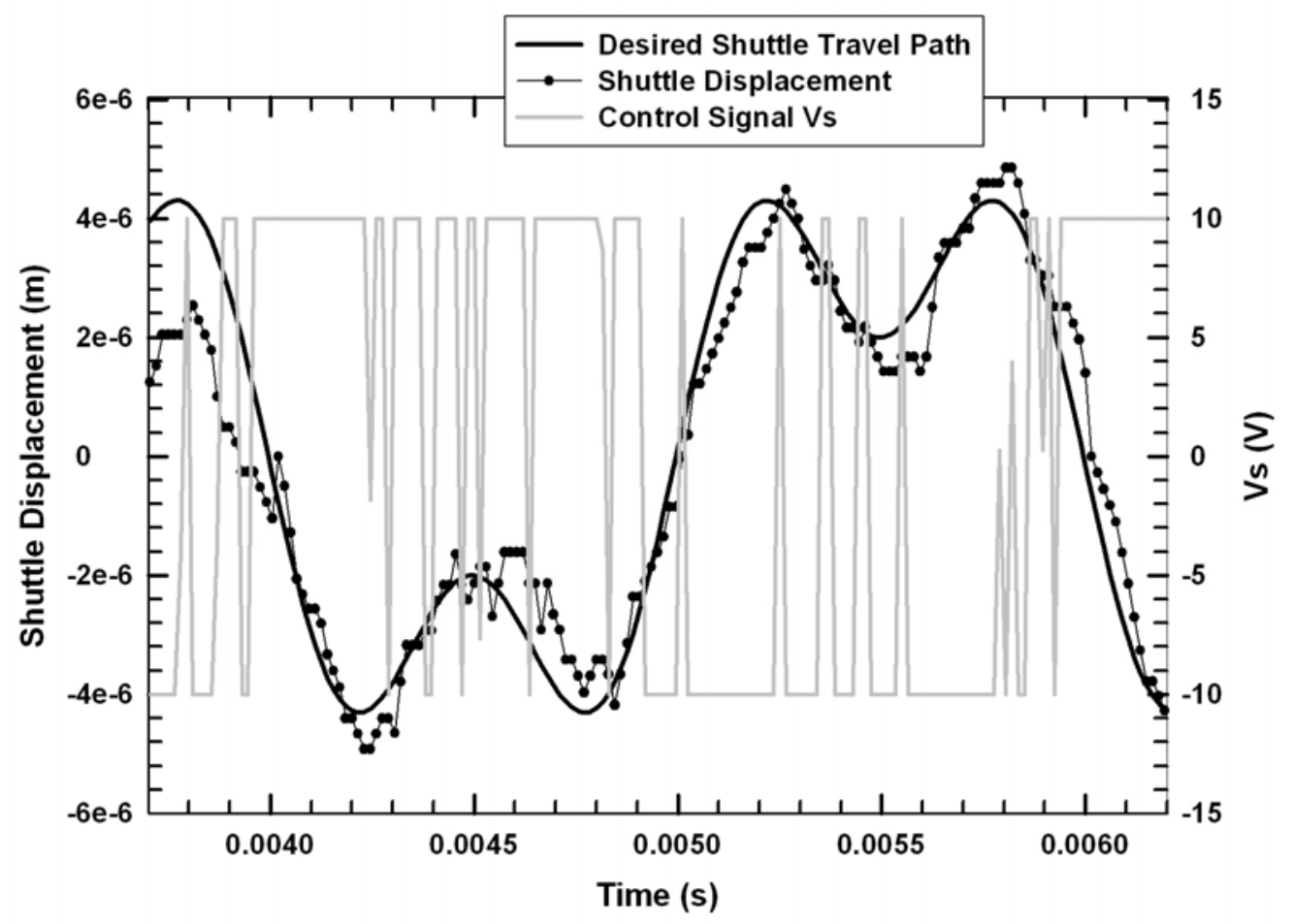

Figure 5-21: Experimental Tracking Results 2

Another tracking control experiment was performed to test if this MFSC controller is robust enough to take care of system failures, and to show how the reliability of a MEMS device can be increased by means of closed-loop control.

In this experiment, system parameter identification was performed on a LCR right after it was released. Then the LCR was put aside for about a month. Due to Poxy used to mount the chip, the temperature, and the moisture, the parameters of the device have changed. As shown in Figure 5-22(a), under the same force condition, when the LCR's system parameters changed due to the environment change, the shuttle displacement also changed in the open-loop operation. However, with the same MFSC controller, even using the reference model built with the original parameters which were estimated right after the device released, the shuttle still can track the desired path (Figure 5-22(b)). 

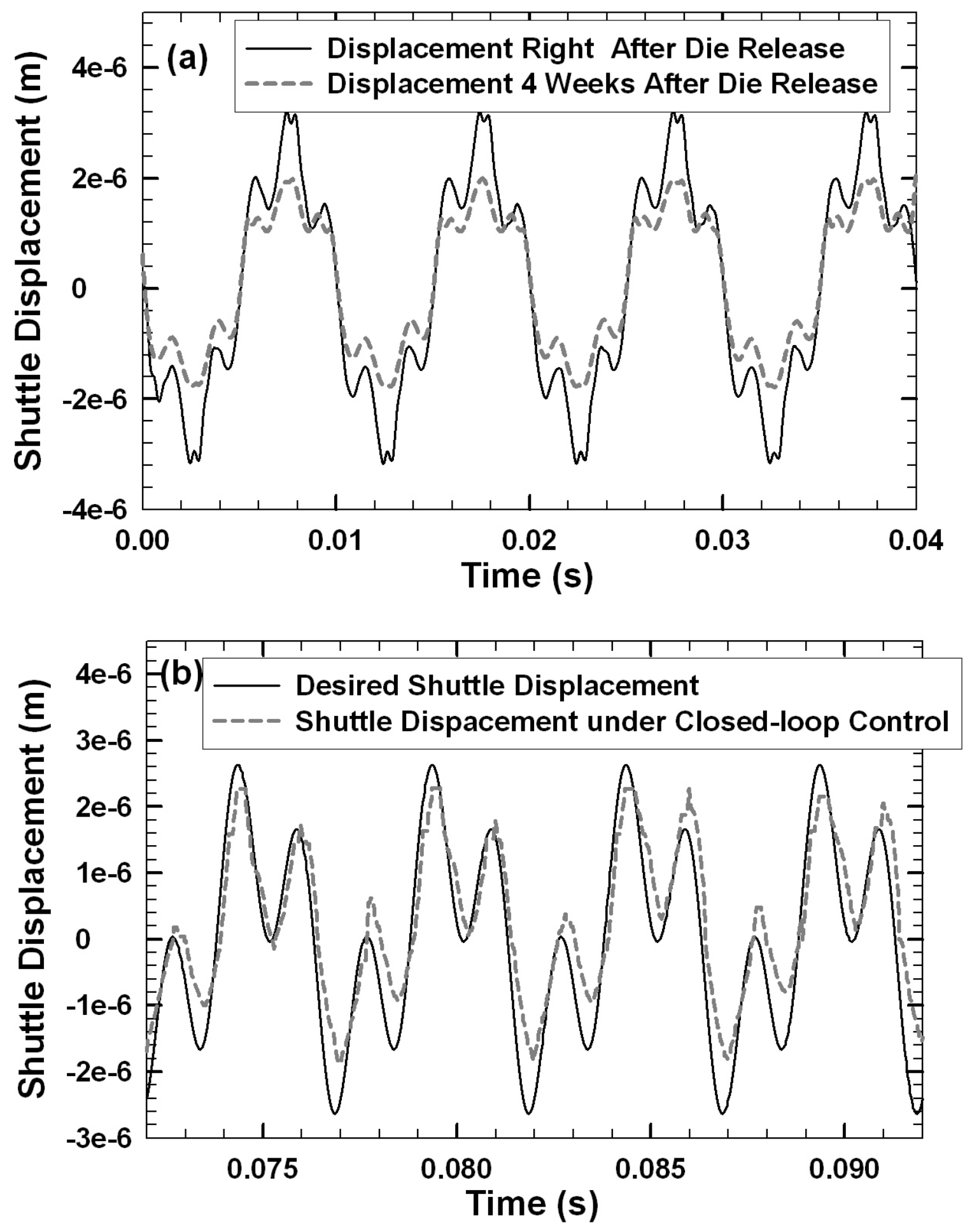

Figure 5-22: (a) The shuttle displacements under same force condition changes due to the change of system parameters; (b) Under Closed-loop control, even the system parameters have changed, shuttle still can follow the desired path. 


\section{Chapter 6 Conclusions and Future Consideration}

\subsection{Conclusions}

The primary goal of this research is to explore the feasibility of increasing the reliability of micro-electro-mechanical system by means of closed-loop control. The research has been focused mainly on the following three areas, and gained insights for the prospective application of real-time feedback control on increasing the reliability and operational flexibility of MEMS devices.

First, the performance of feedback control of any systems is based on the good understanding of the system model because system parameters are required for designing the control system. Compared with macro system, manufacturing tolerance has more effect on microstructures such as MEMS. One major contribution of this work is several experimental methods that were developed to identify the MEMS' model.

Second, the noise level of the optical observing signal is high. The noise was carefully examined, and algorithms that can be used to reconstruct useful position and velocity signals from the encoded signal were developed.

Third, real time feedback control on MEMS LCR was successfully implemented. The successful implementation of tracking control on the MEMS LCR that is described in chapter 5 showed that even when the parameters of the system are changed, the reliability of its operation still can be guaranteed by means of feedback control.

\subsection{Future Consideration}

Current research shows the shuttle of the LCR not only moves in lateral direction, but also has vertical motion, which shows the necessity for building the LCR's 3D model. The possibility of building a mathematical 3D model for the LCR should be surveyed. If no simple mathematical model that is suitable for the control is built, a 3D model with a fuzzy neural network should be developed as a backup solution. Signals that represent the shuttle's movement are necessary in the control effort for the LCR, this makes the online reconstruction of position and velocity signals from the encoded optical monitoring 
signals very important. Currently we have succeeded only in the signal reconstruction on the lateral motion, and with the current algorithm, the noise caused by the optical monitoring technique has to be passed through into the reconstructed signals. Methods that can decouple the optical noise from the reconstructed signal have also been developed using the current single-beam monitoring signal. However, the resolution of this noise decoupled reconstruction signal is only $2 \mu \mathrm{m}$, higher resolution can be achieved by using a multiple-beam monitoring signal and analgorithm based on this signal should be developed in the future. Furthermore, an algorithm that can be used to reconstruct the signals that represent vertical motion also need to be developed. Once the 3D model of the LCR and the real time signals that represent shuttle motion are available, both openloop and real time closed-loop control experiments need to be performed, and the performances of the 3D model control effects need to be compared with that of the current 2D model.

A system identification software package (SISP) and a real-time controller software package (RCSP) should also be developed in the future. The inputs of the SISP will include both the inputs to the LCR and the optical monitoring signal for the LCR's output, while the outputs of the SISP will be the parameters of the system model. There will be two modules in the SISP, the offline module and the on-line module. The on-line module will provide rough system identification results in real time, while the offline module will provide more accurate system identification results. In the offline module, both time domain and frequency domain system identification methods should be performed, and the system identification results can be outputted only when the two results are agree well.

A real-time controller software package (RCSP), which provides different control strategies, should also be developed. The inputs of the RCSP will be the optical monitoring signals, and the outputs will be the voltages that are going to be applied to the LCR. For this software package, the user will have the choice of either inputting the system parameters manually from its interface (the GUI) or importing them from the SISP directly. 


\section{Appendix A Source code \& Simulink model}

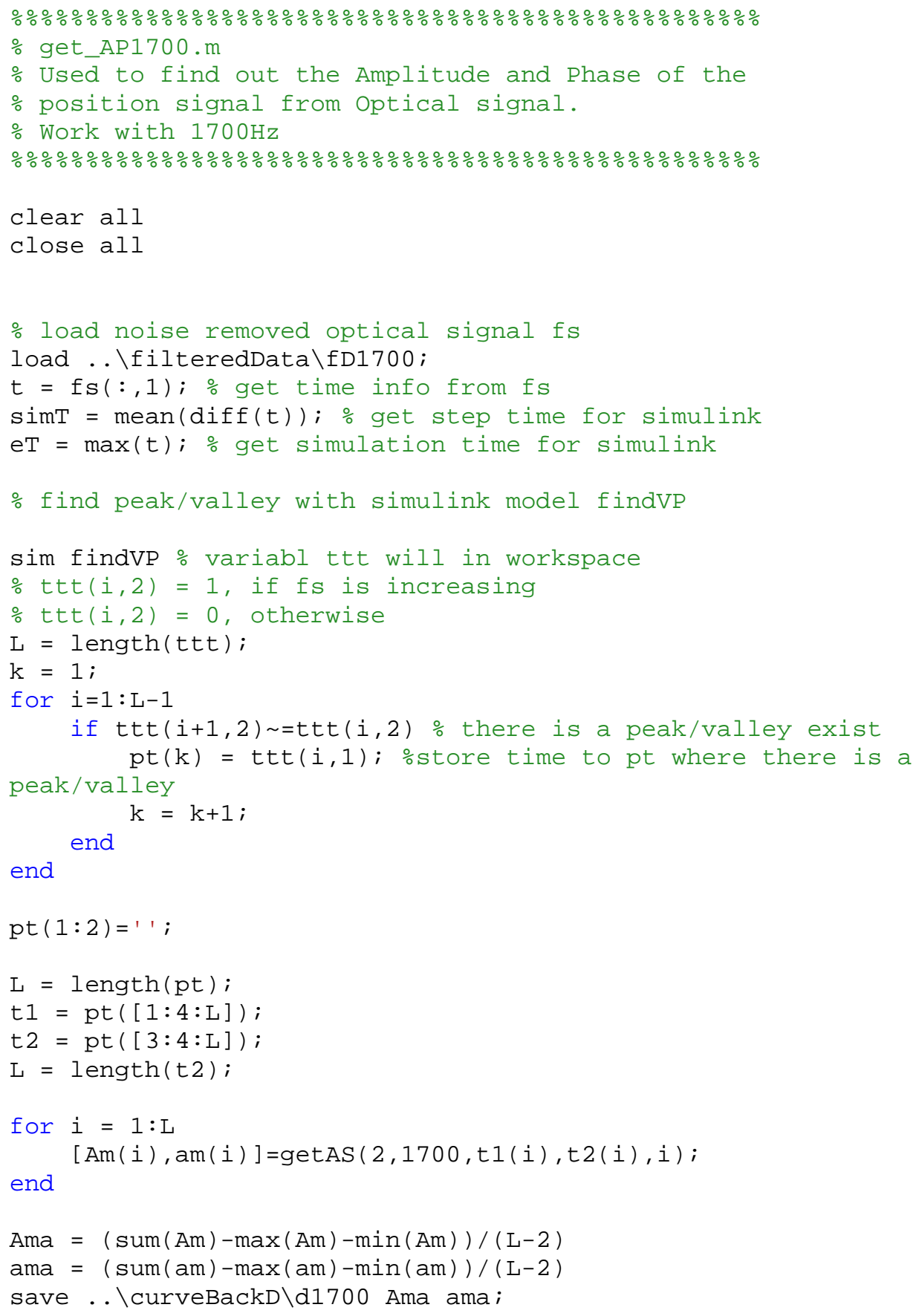




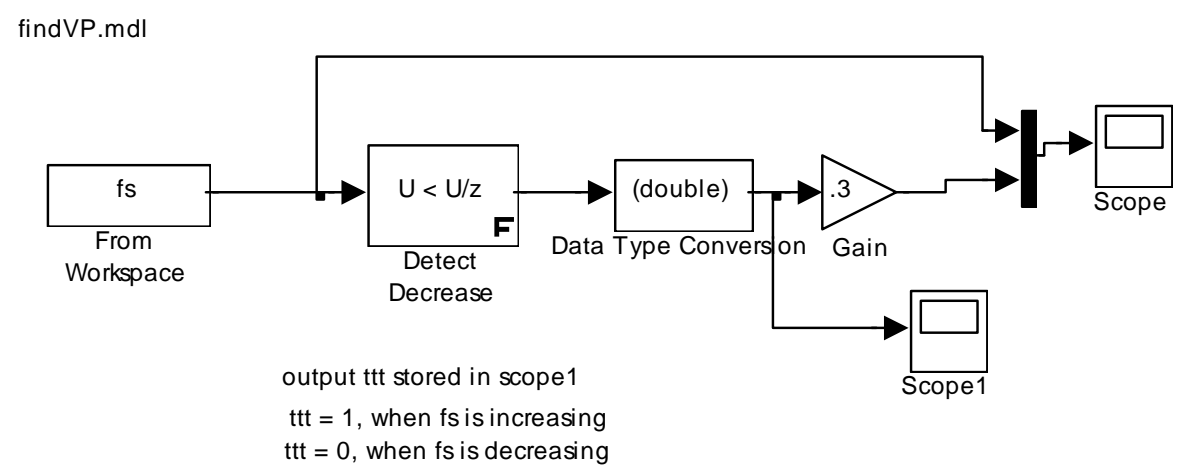

function $[\mathrm{A}$, alfa $]=\operatorname{get} A S(\mathrm{n}, \mathrm{freq}, \mathrm{t} 1, \mathrm{t} 2, \mathrm{k})$

$\%$ calculate amplitude and phase of position signal

$\%$ n number of zero crosses between t1 and t2

$\% \sin ((w(t 1+t 2)+2 a l f a) / 2)=0<--\sin \left(w^{*} t 1+a l f a\right)+\sin \left(w^{*} t 2+a l f a\right)=0$

$\% A=\operatorname{abs}(n * 1 e-6 / \sin (p i * f r e q *(t 2-t 1)))$

alfa $=k^{*}$ pi-pi*freq* $(t 1+t 2)$;

$A=\operatorname{abs}\left(n^{\star} 1 e-6 / \sin \left(2^{*} p^{*}\right.\right.$ freq*t1+alfa $)$; 
\%\%\%\%\%\%\%\%\%\%\%\%\%\%\%\%\%\%\%\%\%\%\%\%\%\%\%\%\%\%\%\%\%\%\%\%\%\%\%\%\%\%\%\%\%\%\%\%

$\%$ gamodel.m, v2.1

$\%$ Using genetic algorithm to identify system parameters

$\%$ model $\mathrm{Fe}=m x^{\prime} '+b x^{\prime}+2 \mathrm{kx}$

$\%$ author Limin Wang

\%\%\%\%\%\%\%\%\%\%\%\%\%\%\%\%\%\%\%\%\%\%\%\%\%\%\%\%\%\%\%\%\%\%\%\%\%\%\%\%\%\%\%\%\%\%\%\%

cwd $=$ pwd;

cd(tempdir);

pack

$\mathrm{cd}(\mathrm{cwd})$

clear all;

close all

load .. Igadata; \%load XA, freq, da

\% xA: Experimental amplitudes of shuttle displacement

$\%$ da: Phase different between experimental shuttle displcement and force

load $\mathrm{Fe} ; \%$ the amplitude of force

$\% \mathrm{GG}=\mathrm{xA} / \mathrm{Fe} ; \%$ calculate the experiment gain

$w=$ freq $^{*}{ }^{*} \mathrm{pi}$;

$\%$ set region of each parameter

c1min $=1.0 \mathrm{e}-10 ; \%$ for $m$

c1max $=3.0 \mathrm{e}-10$;

c2min $=1 \mathrm{e}-7 ; \%$ for beta $\mathrm{b}$

c2max $=1.5 e-6$;

c3min $=.01 ; \%$ for $\mathrm{ks} \mathrm{k}$

c3max $=.04 ;$

c4min $=1 \mathrm{e}-7 ; \%$ for $\mathrm{ks} \mathrm{k}$

c4max $=1 e-4$;

$\mathrm{L}=20$;

$\mathrm{s} 1=(\mathrm{c} 1 \mathrm{max}-\mathrm{c} 1 \mathrm{~min}) /\left(2^{\wedge} \mathrm{L}-1\right) ; \%$ scale rate

s2 $=(\mathrm{c} 2 \mathrm{max}-\mathrm{c} 2 \mathrm{~min}) /\left(2^{\wedge} \mathrm{L}-1\right) ; \%$ scale rate

s3 $=($ c3max $-c 3 m i n) /\left(2^{\wedge} \mathrm{L}-1\right) ; \%$ scale rate

$\mathrm{s} 4=(\mathrm{c} 4 \mathrm{max}-\mathrm{c} 4 \mathrm{~min}) /\left(2^{\wedge} \mathrm{L}-1\right) ; \%$ scale rate

$\mathrm{G}=200 ; \%$ Using $\mathrm{G}$ groups data to implement $\mathrm{GA}$

$\mathrm{NN}=\operatorname{ceil}(\mathrm{G} / 25)$;

bt1 $=\operatorname{zeros}(G, 1) ; \% \mathrm{~m}$

$\mathrm{bt2}=\mathrm{bt} 1 ; \% \mathrm{~b}$

bt3 = bt1; \%ks

bt4 = bt1; \%rt, the rate between optical signal $\$$ position signal

$\mathrm{c1}=\operatorname{lrand}(\mathrm{G}, \mathrm{L}) ; \%$ initial $\mathrm{G}$ groups $\mathrm{c} 1$

$\mathrm{C} 2=\operatorname{lrand}(\mathrm{G}, \mathrm{L}) ; \%$ initial $\mathrm{G}$ groups $\mathrm{C} 2$

$c 3=\operatorname{lrand}(G, L) ; \%$ initial $G$ groups $c 3$

$\mathrm{c} 4=\operatorname{lrand}(\mathrm{G}, \mathrm{L}) ; \%$ initial $\mathrm{G}$ groups $\mathrm{c} 4$

$\mathrm{f}=1$; \% set flag, will be cleared when coverge

$\mathrm{ksp}=0$;

while $f$

$\% \mathrm{ksp}=\mathrm{ksp}+1$

$\%$ convert c from bin. to dec.

for $i=1: G$

$b t 1(i)=b 2 d(c 1(i,:))$; 


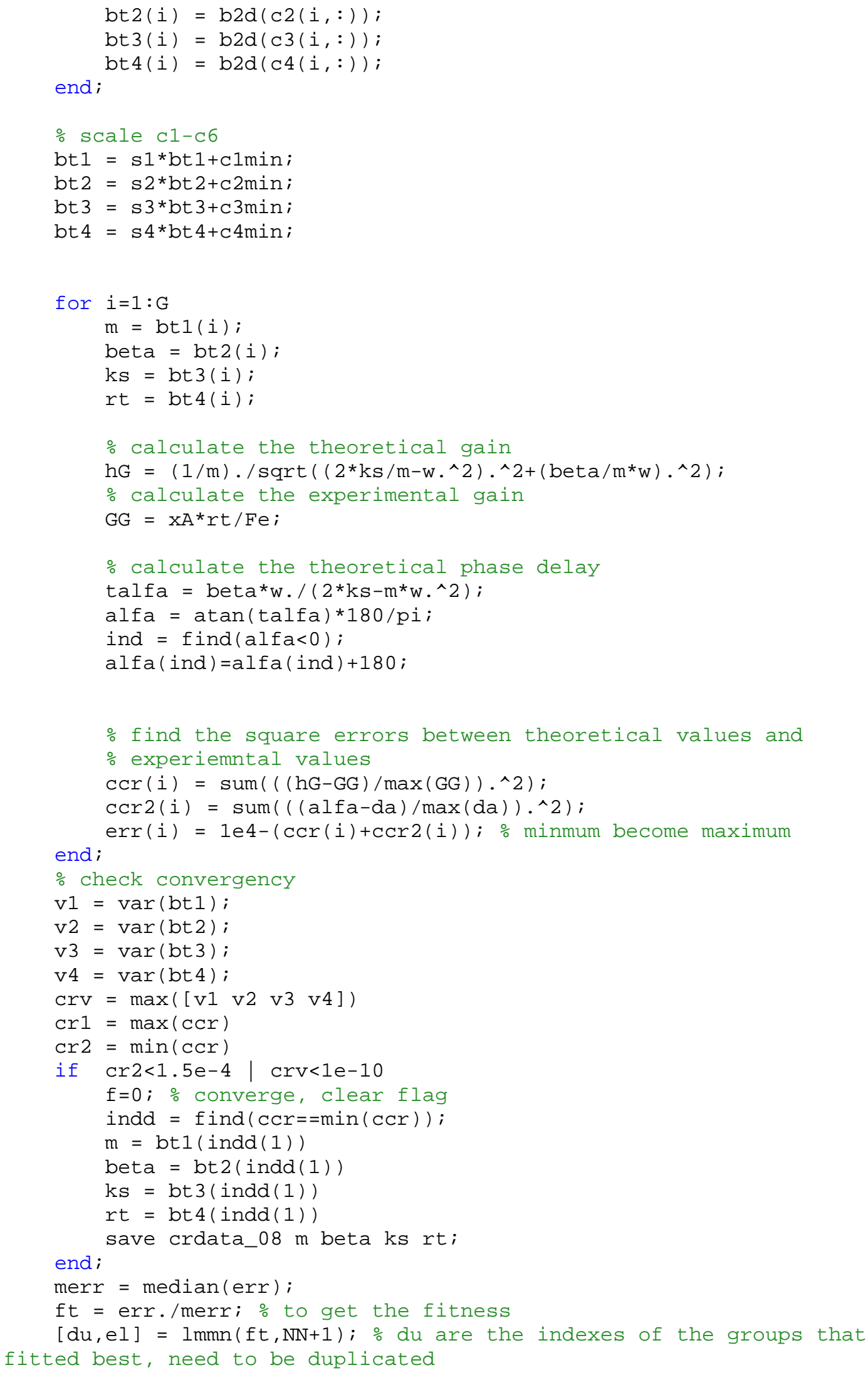


$\%$ while el are the indexes of the group that have the worse match condition, need to be eliminated

$\%$ substitude the weakest group by strongest one

$c 1(e l,:)=c 1(d u,:)$;

c2 $(\mathrm{el},:)=\mathrm{c} 2(\mathrm{du},:)$;

$\mathrm{c} 3(\mathrm{el},:)=\mathrm{c} 3(\mathrm{du},:)$;

$c 4(e l,:)=c 4(d u,:)$;

$\%$ do the cross over to reproduce new groups

for $i=1: N N$

$\mathrm{k}=\operatorname{lseq}(\mathrm{G}) ; \%$ generate a random sequency, in order to do cross over randomely

$\mathrm{p}=\operatorname{lrandb}(1, \mathrm{~L}-1) ; \%$ randomly choose a place in the groupe $\mathrm{t} 1=\mathrm{c} 1(\mathrm{k}(\mathrm{i}), \mathrm{p}+1: \mathrm{L})$;

$c 1(k(i), p+1: L)=c 1(k(G-i), p+1: L)$;

$\mathrm{c} 1(\mathrm{k}(\mathrm{G}-\mathrm{i}), \mathrm{p}+1: \mathrm{L})=\mathrm{t} 1$;

$\mathrm{k}=\operatorname{lseq}(\mathrm{G}) ; \%$ generate a random sequency, in order to do cross over randomely

$\mathrm{p}=\operatorname{lrandb}(1, \mathrm{~L}-1) ; \%$ randomly choose a place in the groupe $\mathrm{t} 2=\mathrm{c} 2(\mathrm{k}(\mathrm{i}), \mathrm{p}+1: \mathrm{L})$;

$c 2(k(i), p+1: L)=c 2(k(G-i), p+1: L)$;

$\mathrm{c} 2(\mathrm{k}(\mathrm{G}-\mathrm{i}), \mathrm{p}+1: \mathrm{L})=\mathrm{t} 2$;

$\mathrm{k}=\operatorname{lseq}(\mathrm{G}) ; \%$ generate a random sequency, in order to do cross over randomely

$p=\operatorname{lrandb}(1, L-1) ; \%$ randomly choose a place in the groupe $\mathrm{t} 3=\mathrm{c} 3(\mathrm{k}(\mathrm{i}), \mathrm{p}+1: \mathrm{L})$;

$c 3(k(i), p+1: L)=c 3(k(G-i), p+1: L)$; $c 3(k(G-i), p+1: L)=t 3$;

$\mathrm{k}=\operatorname{lseq}(\mathrm{G}) ; \%$ generate a random sequency, in order to do cross over randomely

$\mathrm{p}=\operatorname{lrandb}(1, \mathrm{~L}-1) ; \%$ randomly choose a place in the groupe $\mathrm{t} 4=\mathrm{c} 4(\mathrm{k}(\mathrm{i}), \mathrm{p}+1: \mathrm{L})$;

$c 4(k(i), p+1: L)=c 4(k(G-i), p+1: L)$; $c 4(k(G-i), p+1: L)=t 4 ;$

end; end;

load . . Igadata;

$\mathrm{GG}=\mathrm{xA} * \mathrm{rt} / \mathrm{Fe} ; \%$ calculate the experiment gain

$w=$ freq $^{*} 2^{*} p i$;

$w 2=2^{*} p i^{*}(20: 20: 4000) ;$

hG2 $=(1 / \mathrm{m}) \cdot / \operatorname{sqrt}\left((2 * \mathrm{ks} / \mathrm{m}-\mathrm{w} 2, \wedge 2) \cdot \wedge 2+\left(\right.\right.$ beta $\left.\left./ \mathrm{m}^{*} \mathrm{w} 2\right) \cdot \wedge 2\right) ;$

str $=\operatorname{strcat}\left(' m={ }^{\prime}, \operatorname{num} 2 \operatorname{str}(m),{ }^{\prime}, k s=', \operatorname{num} 2 \operatorname{str}(k s),{ }^{\prime}\right.$,

beta ${ }^{\prime}$, num2str(beta) );

subplot $(2,1,1), p \operatorname{lot}\left(w / 2 / p i, 10 * \log 10(G G),{ }^{*}\right.$ ' $\left., w 2 / 2 / p i, 10 * \log 10(h G 2)\right)$;

xlabel('frequency $(\mathrm{Hz})$ ');

ylabel('gain $(\mathrm{dB})$ ')

legend('Experiment $\mid$ Fe $|/| x \mid$ ', 'Curve Result');

$\operatorname{text}(800,12, \mathrm{str})$

talfa $=$ beta*$^{*} 2 . /\left(2{ }^{*} \mathrm{ks}-\mathrm{m}^{*} \mathrm{w} 2 . \wedge 2\right)$;

alfa $=\operatorname{atan}(\mathrm{talfa}) * 180 / \mathrm{pi}$;

ind $=$ find $($ alfa $<0)$;

alfa $($ ind $)=\operatorname{alfa}($ ind $)+180$; 


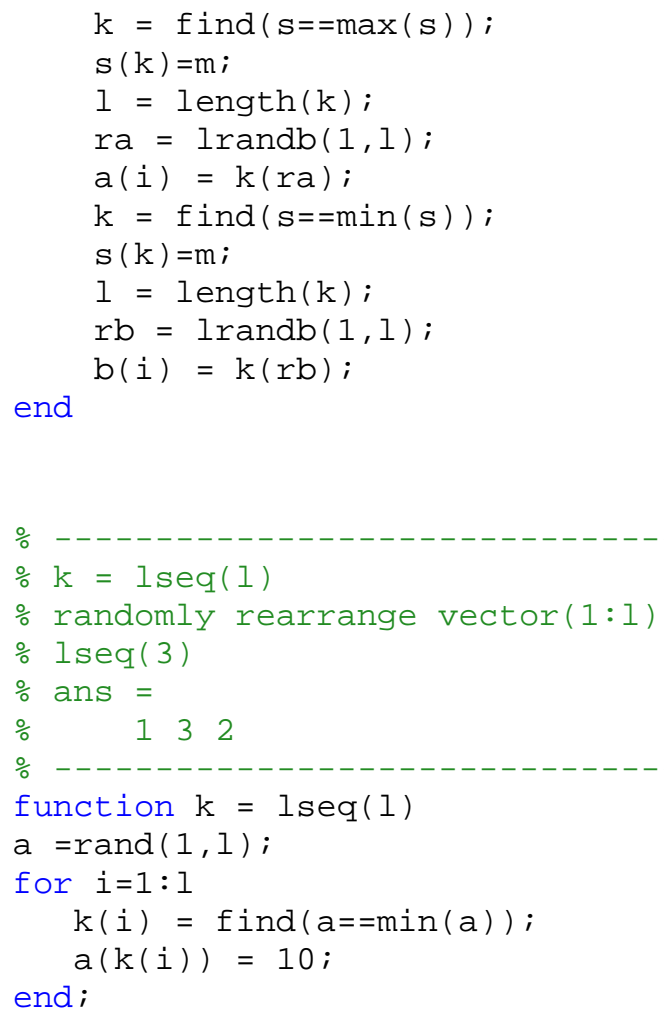


Stroke Length Control

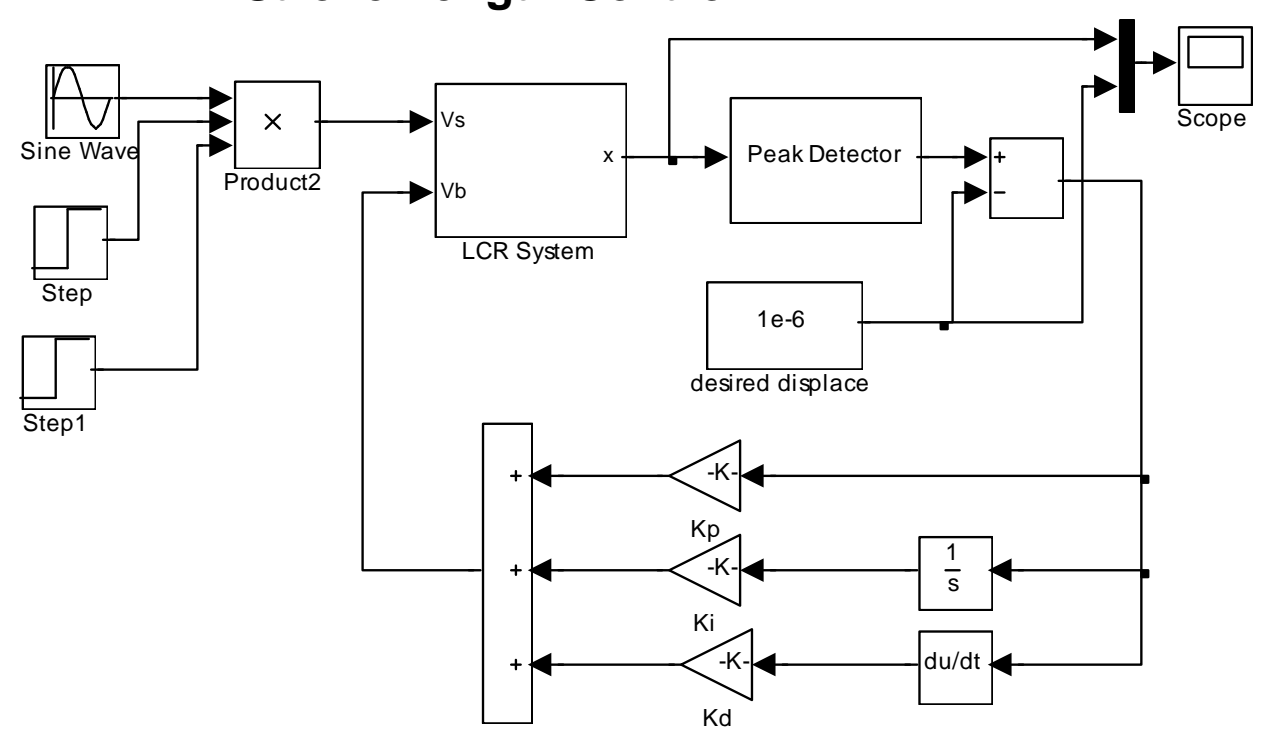

Peak Detector

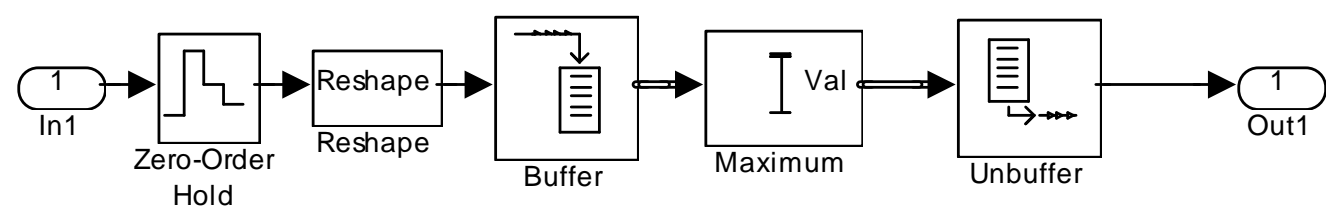



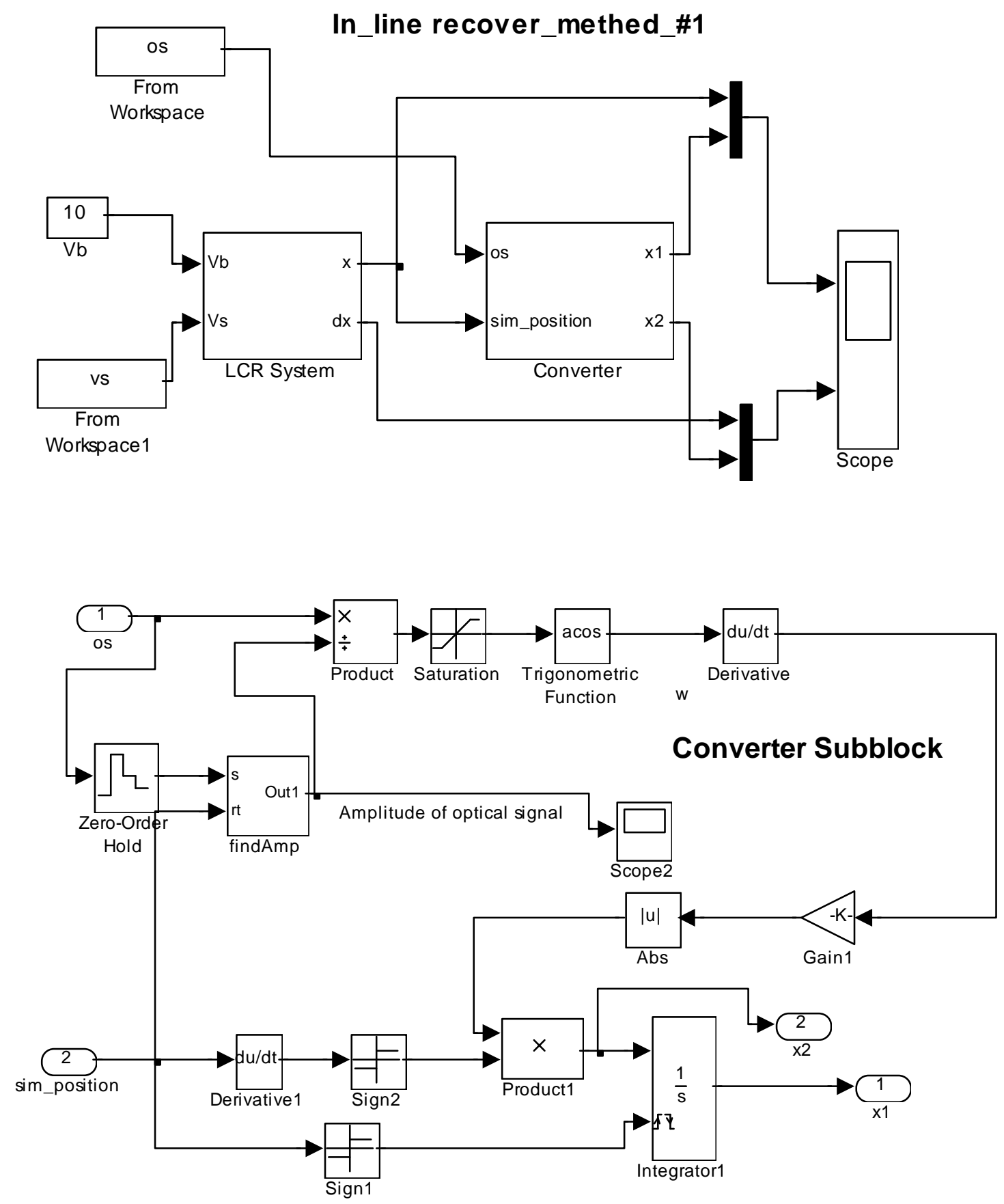


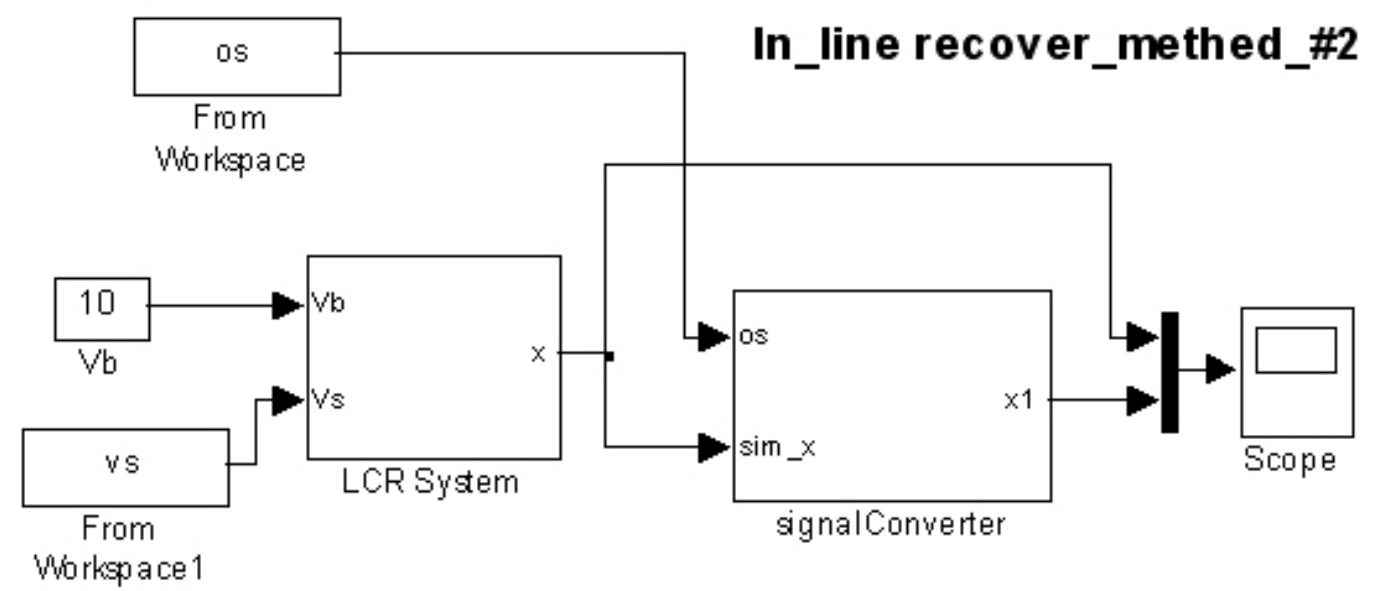

Signal Converter Unit

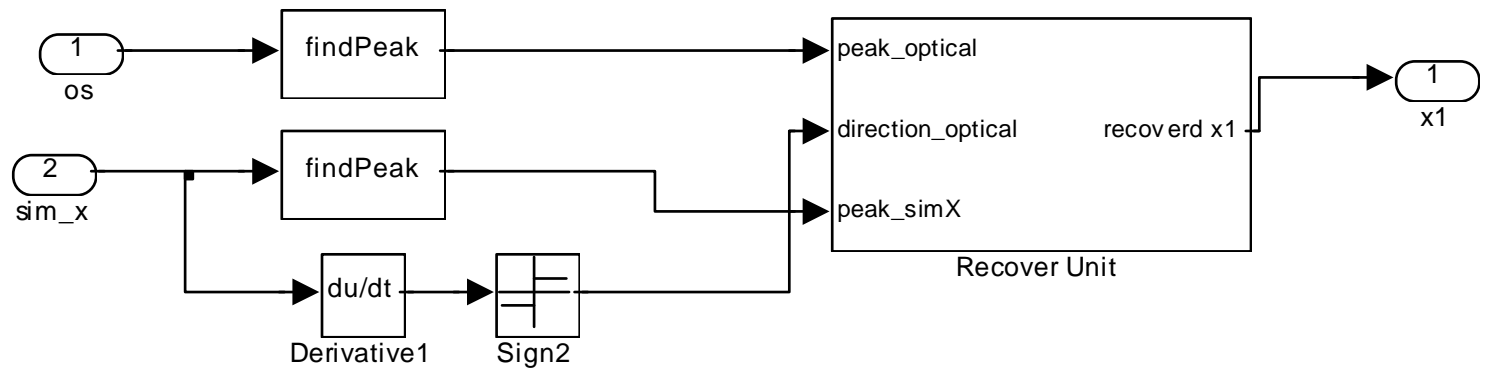

Find Peak

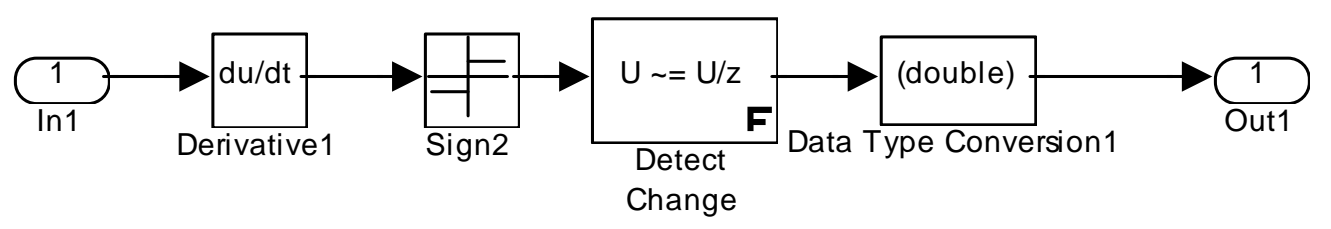




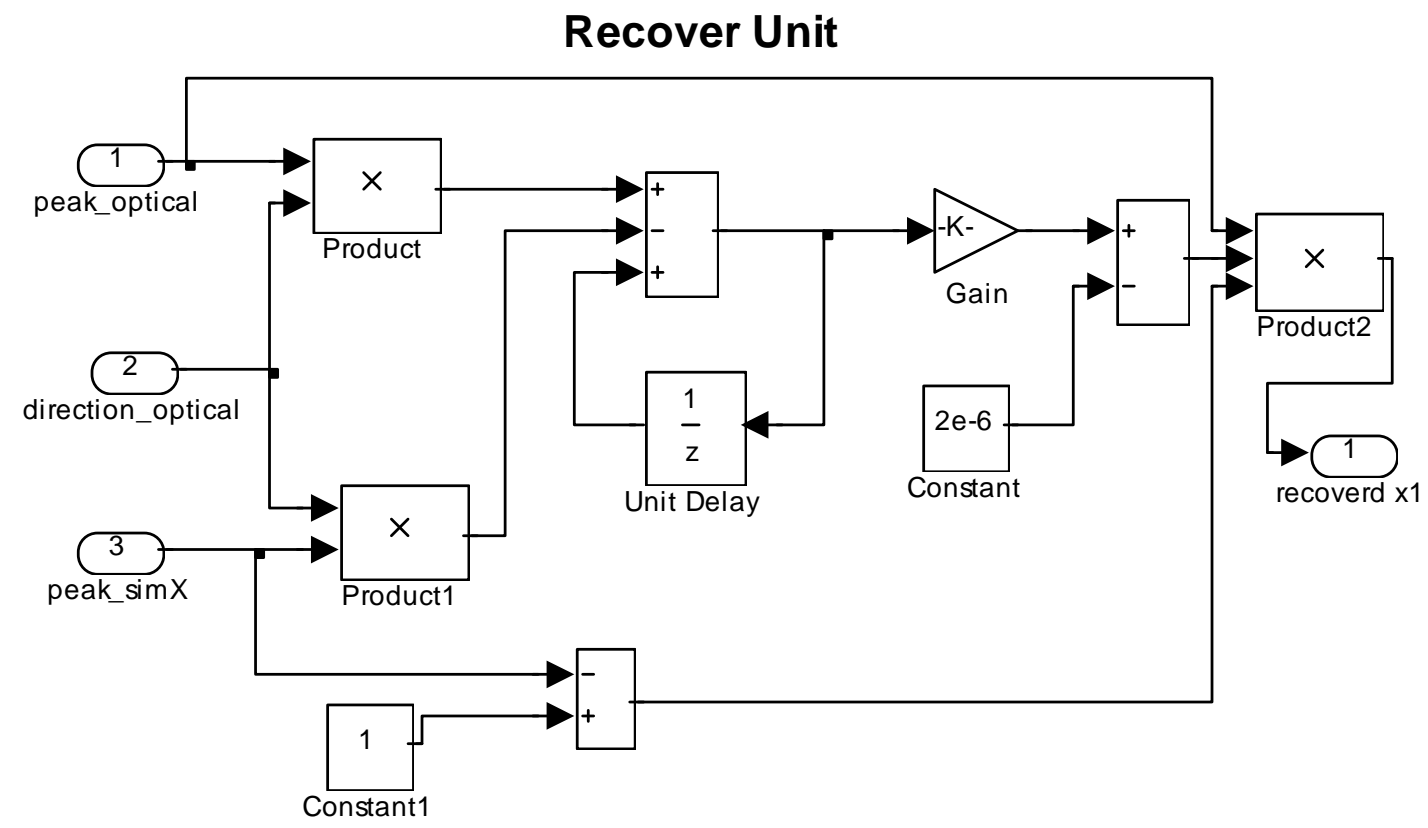




\section{Bibliography}

[1] J.M. Dawson, L. Wang, W.B. McCormic, S.A. Rittenhouse, P. Famouri, and L.A. Hornak, "Integrated optical monitoring of MEMS for closed loop control," in Proc. of SPIE Micromachining and Microfabrication Symposium, v 4983, pp 34-43, Jan. 2003, Santa Clara CA

[2] L. Wang, J.M. Dawson, J. Chen, P. Famouri, and L.A. Hornak, "Stroke-length control of a MEMS device," in Proc. of the 2000 IEEE International Symposium on Industrial Electronics, pp 535-539. Dec. 48, 2000, Cholula, Puebla, Mexico.

[3] L. Wang, J.M. Dawson, L.A. Hornak, and P. Famouri, “Applied real-time high-frequency closed-loop control of a MEMS lateral comb resonator,” IEEE Trans. Aerospace and Electronic Systems, vol.40, pp.567-575, Apr. 2004.

[4] J. Park, L. Wang, J.M. Dawson, L.A. Hornak, and P. Famouri, "Microstructure torque estimation using MEMS optical monitoring,” in Proc. of IEEE Sensors 2002, June 12-14, 2002, Orlando, Florida, USA.

[5] D. J. Nagel, and M. E. Zaghloul, “MEMS: Micro Technology, Mega Impact,” IEEE Circuits and Devices, vol.17, no. 2, pp. 14-25, March 2001.

[6] http://www.sandia.gov

[7] R. C. Jaeger, “Introduction to microelectronic fabrication,” Modular Series on Solid State Deveices, vol. V, Editors: G. W. Neudeck, and R. F. Pierret, Addision-Wesley Publishing Company, Inc. 1998.

[8] J.W. Judy, “Microelectronmechanical systems (MEMS): fabrication, design and applications,” Smart Materials and Structures, v10, n6, pp. 1115-1134, Dec. 2001.

[9] D. Koester, A. Cowen, R. Mahadevan, and B. Hardy, “PolyMUMPs design handbook,” reversion 8.8, by MEMSCAP

[10] http://mems-ex.cnri.reston.va.us/MEMS/challenges.html

[11] S.L. Miller, M.S. Rodgers, G. La Vigne, J.J. Sniegowski, P. Clews, D.M. Tanner, and K.A. Peterson, "Failure modes in surface micromachined microelectromechanical actuators," Microelectronics and Reliability, v39, n8, 1999, Elseveier Science Ltd Exeter Engl., pp 1229-1237.

[12] A.T. Echtermeyer, B. Engh, and L. Buene, "Lifetime and Young's modulus changes of glass/phenolic and glass/polyester composites under fatigue,” Composites, v 26, n 1, Jan, 1995, pp 10-16.

[13] B.D. Jensen, K. Saitou, J.L. Volakis, and K. Kurabayashi, "Impact of skin effect on thermal behavior of RF MEMS switches,” submitted to The 6th ASME-JSME Thermal Engineering Joint Conference,March 16-20, in review.

[14] A. T. DiBenedetto, “The structure and properties of materials,” McGraw-Hill, United States of America, 1967.

[15] S. C. Jacobsen, R. H. Price, J. E. Wood, T. H. Rytting, and M. Rafaelof, "The wobble motor: an electrostatic, planetary armature, microactuator” in Proc. of IEEE Microelectromechanical Systems, pp. 1724, February 1989.

[16] C. Burroughs, "Sandia's precision MEMS reliability tests advance future of micromachine systems," http://www.sandia.gov/LabNews/LN06-05-98/mems_story.html 
[17] B. Stark, “MEMS reliability assurance guidelines for space applications,” Editor: Jet Propulsion Laboratory, Pasadena, California, http://nppp.jpl.nasa.gov/resinfo_refmaterials.htm

[18] K. P. Larsen, J. T. Ravnkilde, M.Ginnerup, and O. Hansen, "Device for fatigue testing of electroplated nickle (MEMS),” in Proc. of MEMS 2002, pp. 443-446, Las Vegas, January 2002.

[19] S. Bhansali, A.L. Zhang, R.B. Zmood, P.E. Jones, and D.K. Sood, "Prototype feedback-controlled bidirectional actuation system for MEMS applications," Journal od Microelectromechanical systems, v 9, no 2, June, 2000, pp. 245-251.

[20] Y. Li and R. Horowitz, "Active suspension vibration control with dual stage actuators in hard disk drives," in Proc. of American Control Conference, Arlington, VA June 25-27, 2001, pp. 2786-2791.

[21] Pannu, S., Chang, C., Muller, R.S., and Pisano, A.P., "Closed-loop feedback control systems for improved tracking in magnetically actuated micromirrors,” 2000 IEEE/LEOS International Conference on Optical MEMS, Aug. 21-24, 2000, Kauai, HI,USA, pp 107-108.

[22]Chen, Y. Shroff, Y., and Oldham, W.G., "Modeling and control of nanomirrors for EUV maskless lithography," Proceedings of the 2000 International conference on modeling and simulation of microsystems, San Diego, california, USA, March 27-29, 2000, pp. 602-604.

[23] Lyshevski, Sergey Edward, "Integrated control of microactuators and integrated circuits: a new turning approach in MEMS technology," Proc. of the IEEE Conference on Decision and Control, V3, 1999, Dec 710, 1999, Phonix, AZ, USA, pp. 2611-2616.

[24] G. Abadal, Z.J. Davis, B. Helbo, X. Borrisé, R. Ruiz, A. Boisen, F. Campabadal, J. Esteve, E. Figureras, F. Pérez-Murano, and N. Barniol, "Electromechanical model of a resonating nano-cantileverbased sensors for high-resolution and high-sensitivity mass detection,” Journal of Nanotechnology, v.12, 2001, UK, pp 100-104.

[25] J.I. Seeger, and B.E. Boser, "Dynamics and control of parallel-plate actuators beyond the electrostatic instability,” Transducers, 99', The 10th International Conference on solid-slState Sensors and Actuators, Sendai, Japan, June 7-9, 1999, pp 474-477.

[26] J.A. Pelesko, and A.A. Trolo, "Nonlocal problems in MEMS device control," Proc. of the 2000 International conference on modeling and simulation of microsystems, San Diego, california, USA, March 27-29, 2000, pp. 509-512.

[26] J. Ernest, J. Garcia and J.J. Sniegowski, “Surface micromachined microengine”, IEEE Electron Device Letters, Vol. 17, No. 7, 366, July 1996.

[27] J. Jeffry, J. Sniegowski, S.L. Miller, F. Glenn, M. LaVigne, R. Steven, and P. J. McWhorter, "Monolithic geared-mechanisms driven by a polysilicon surface-micromachined on-chip electrostatic microengine”, Solid-State Sensor and Actuator Workshop, Hilton Head Is., SC, June 2-6, 1996, pp. 178182.

[28] S.E. Lyshevski, “Micro-electromechanical systeme: motion control of micro-actuators”, Proc. of the 37th IEEE conference on Decision and Control: December 16-18, 1998, pp. 4334-4335.

[30] D.M. Freeman, “Computer microvision for MEMS,” Proc. Material Research Society Symposium, v605, 2000, Materials Science of Microelectromechanical System (MEMS) Devices II, Nov 29-Dec1 1999, Boston, MA, USA, pp. 151-162. 
[31] M.R. Hart, R.A. Conant, K.Y. Lau, and R.S. Muller, "Stroboscopic interferometer systems for dynamic MEMS characterization,” Journal of Microelectromechanical Systems, v9, no.4, pp. 409-418, December 2000.

[32] C. Rembe, R. Kant, and R. Muller, "Optical measurement methods to study dynamic behavior in MEMS,” http://www-bsac.eecs.berkeley.edu/projects/memstesting/SPIEPaper4400_15.pdf

[33] Dickey, Holswade, Christenson, Garcia, and Polosky, “Optical measurement of LIGA milliengine performance,” in Proc. SPIE Miniaturized Sys. With Micro-Optics and Micromechanics III, Vol. 3276, pp. 28-36 (1998).

[34] E.J. Garcia and J. Sniegowski, “Surface micromachined microengine,” Sensors and Actuators A: Physical, Vol. 48(3), pp. 203-14 (1995).

[35] J.M. Dawson, J. Chen, K.S. Brown, P. Famouri, L.A. Hornak, "Through-wafer optical probe characterization for microelectromechanical systems positional state monitoring and feedback control," Optical Engineering, vol.39, no 12, pp 3239-3246, Dec. 2000.

[36] L.A. Hornak, P. Famouri, J.M. Dawson, L. Wang, and R. Ghaffarian, "MOEMS integrated optical monitoring,” Proc. of SPIE, MOEMS and Miniaturized Systems II, vol. 4561, pp. 221-230, Oct. 2001.

[37] J. Dawson, L. Wang, W. McCormick, S. Rittenhouse, P. Famouri, L. A. Hornak, "Integrated Optical Monitoring of MEMS for closed Loop Control," to appear in Proc of SPIE Micromachining and Microfabrication Symposium, (Santa Clara CA, Jan. 2003).

[38] F. M. DICKEY, S. C. HOLSWADE, L. A. HORNAK, and K. S. BROWN: 'Optical Methods for Micromachine Monitoring and Feedback’, Sensors and Actuators A, Phys, 1999, 78, p. 220.

[39] J. Shao, and V. Cherkassky, "Improved VC-based signal denoising," Proc. of the International Joint Conference on Neural Networks, v 4, 2001, International Joint Conference on Neural Networkss (IJCNN’01), Jul 15-19 2001, Washington, DC, pp. 2439-2444.

[40] http://www-mrips.od.nih.gov/faq/s_smoothing.html

[41] A.R. Weeks, "Fundamentals of electronic image processing," SPIE Optical Engineering Press and IEEE Press, (1996).

[42] S. Bal, W. Kinsner, "Image denoising for reduced-search fractal block coding," Canadian Conference on Electrical and Computer Engineering, v 1, 1996, Proceedings of the 1996 Canadian Conference on Electrical and Computer Engineering, Part 1 (of 2), May 26-29, Calgary, Can, pp 438-441.

[43] P. Quick and D. Capson, “Analysis of determining camera position via Karhunen-Loeve transform,” in Proc. of the 4th IEEE Southwest Symposium on Image Analysis and Interpretation. Apr. 2000, pp 88-92.

[44] M. Fleury, A.C. Downton, A.F. Clark, "Karhunen-Loeve transform: An exercise in simple imageprocessing parallel pipelines,” Lecture Notes in Computer Science, v1300, 1997, pp. 815.

[45] B. Aysin, L.F. Chaparro, I. Grave, and V. Shusterman, “Denoising of non-stationary signals using Karhunen-Loeve expansion," Proc. IEEE-SP Intl. Symp. on Time-Frequency and Time-Scale Analysis, Pittsburgh, pp. 621-624, Oct. 1998

[46] Y. Yamashita, Y. Ikeno and H. Ogawa, "Relative Karhunen-Loeve transform” IEEE Transactions on Signal Processing, v44, n2, Feb, 1996, p 371-378

[47] L. P. Yaroslavsky, "Linear and rank adaptive filters for image processing, digital image processing and computer grasphics,” Theory and Applications, 1991, pp. 374-400 
[48] L.P. Yaroslavsky, "Local adaptive image restoration and enhancement with the use of DFT and DCT in a running window," Wavelet Applications in Signal and Image Processing IV, SPIE vol. 2825, pp. 2-11, 1996

[49] H. S. Malvar, Signal processing with Lapped transforms, Boston, MA: Artech House, 1992.

[50] Z. Xiong, and H.S. Malvar, “A nonuniform modulated complex Lapped transform,” IEEE Signal Processing Letters, vol. 8, no. 9, Sep. 2001, pp. 257-260.

[ 51 ] R. Polikar, "The engineer's ultimate guide to wavelet analysis - the wavelet tutorial," http://engineering.rowan.edu/\%7Epolikar/WAVELETS/WTtutorial.html.

[52] C. Taswell, "The what, how, and why of wavelet shrinkage denoising," IEEE Computeing in Science \& Engineering, vol 2, no 3, May/June, 2000, pp, 12-19.

[53] D.L. DohonoL. and I.M. Johnston, “Ideal spatial adaptation via wavelet shrinkage,” Biometrika, v 81, 1994, pp. 425-455.

[54] F. M. Dickey, S. C. Holswade, L. A. Hornak, and K. S. Brown: "Optical methods for micromachine monitoring and feedback,” Sensors and Actuators, Phys, 1999, 78, pp. 220.

[55] J. J. Sniegowski and E. J. Garcia, "Microfabricated actuators and their application to optics”, SPIE Vol. 2383, pp. 46-64.

[56] N. Deb, S.V. Iyer, T. Mukherjee, and R.D. Blanton, "MEMS resonator synthesis for defect reduction," Journal of Modeling and Simulation of Microsystems, vol.2, no.1, 2001, pp. 11-20.

[57] A. Kolpekwar and R. D. Blanton, "Development of a MEMS testing methodology," IEEE International Test Conference, pp. 923-31, 1997.

[58] C.G. Koh, B. Hong, and C.Y. Liaw, "Parameter identification of large structural systems in time domain,” Journal of Structural Engineering, v 126, n 8, Aug, 2000, pp. 957-963.

[59] N. Zhang, and S. Hayama, "Identification of structural system parameters from time domain data (direct identification of mass, stiffness and damping parameters of a structure)," JSME International Journal, Series 3: Vibration, Control Engineering, Engineering for Industry, v 34, n 1, Mar, 1991, pp 6471.

[60] L. Wang, S. Shen, S. Zhu, and F. Zhou, "Method of time domain identification based on EE model for the excitation system parameters," Dianli Xitong Zidonghue/Automation of Electric Power Systems, v 26, n 8, Apr 25, 2002, pp 25-28,37.

[61] J.K. Pinkelman, S.M. Batill, and M.W. Kehoe, "Investigation of the total least-squares criteria in time domain based, parameter identification for flight flutter testing," Collection of Technical Papers AIAA/ASME/ASCE/AHS/ASC Structures, Structural Dynamics \& Materials Conference, n 2, 1995, pp 783793.

[62] E.S. Boje, J.C. Balda, R.G. Harley, and R.C. Beck, “Time-domain identification of synchronous machine parameters from simple standstill tests,” IEEE Trans. Energy Conversion, v 5, n 1, Mar, 1990, pp 164-175.

[63] W. Tang, Z. Liang, and X. Zhen, "New time domain method for identification of modal parameters and its application,” Zhendong Ceshi Yu Zhenduan/Journal of Vibration, Measurement \& Diagnosis, v 18, n 3, Sep, 1998, pp 190-196. 
[64] A. M. Iglesias, "Investigating various modal analysis extraction techniques to estimate damping ratio,” thesis, Virgina Polytechnic Institute and State University, June29, 2000.

[65] W. S. Levine, The control handbook, CRC Press and IEEE Press, 1996.

[66] L.D. Peterson, and K.F. Alvin, "Time and frequency domain procedure for identification of structure dynamic models,” Journal of Sound and Vibration, 1988 201(1), pp. 137-144.

[67] R. Pintelon, and J. Schoukens, System identification, a frequency domain approach, IEEE Press, 2001. [68] N.K. Sinha, "System identification: from frequency response to soft computing," Proc. of the IEEE International Conference on Industrial Technology 2000, Jan 19-22, 2000. pp 76-80.

[69] L. Ljung, "Some results on identifying linear systems using frequency domain data," Proc. of the $32^{\text {nd }}$ IEEEE Conference on Decision an Control, Dec 15-17, 1993, pp. 3534-3538.

[70] X. Wang, and H. Shao, “A novel method of frequency domain identification for SISO system based on improved two-channel relay feedback,” Proc. of the American Control Conference, June 25-27, 2001, pp. 3286-3287.

[71] R. Printelon,, "Frequency domain subspace system identification using non-parametric noise models," Proc. of the $40^{\text {th }}$ IEEE Conference on Decision and Control, Dec. 2001, pp. 3916-3921.

[72] R. Printelon, J. Schoukens, and G. Vandersteen,, "Frequency domain system identification using arbitary signals,", Proc. of the $325^{\text {th }}$ Conference on Decision and Control, Dec. 1995, pp. 2048-2051.

[73] G.K. Fedder, "Simulation of electromechanical system,” Ph.D. thesis, university of California at Berkeley, 1994.

[ 74 ] R.R. Medipalli, "MEMS parallel-plate electrostatic actuator: modeling, simulation and characterization,” Master thesis, West Virginia University, 2004.

[75] L. Wang, J.M. Dawson, J. Chen, P. Famouri, and A. Hornak, "Stroke-length control of a MEMS device,” Proc. of the 2000 IEEE International Symposium on Industry Electronics, ISIE 2000, Mexico, dec 408, 2000, pp535-539.

[76] J. Dawson, L. Wang, W. McCormick, S. Rittenhouse, P. Famouri, L. A. Hornak, "Integrated optical monitoring of MEMS for closed loop control," to appear in Proc of SPIE Micromachining and Microfabrication Symposium, (Santa Clara CA, Jan. 2003).

[77] W. McCormick, Master thesis, West Virginia University, 2004

[78] S. V. Iyer, H. Lakdawala, T. Mukherjee and G. Fedder, "Modeling methodology for a CMOS-MEMS electrostatic comb”, in Design, Test, Integration and Packaging of MEMS/MOEMS (DTIP '02), pp. 114125, (Cannes, France, May 6-8, 2002).

[79] J. Chen, P. Famouri and L. A. Hornak, "Nonlinear control of MEMS: micorengine sliding mode control simulation”, Proc. of the IASTED International Conference, Oct 28-30, 1999, pp. 96-101. 